\title{
Mirror symmetry and loop operators
}

\author{
Benjamin Assel ${ }^{a}$ and Jaume Gomis ${ }^{b}$ \\ a Department of Mathematics, King's College London, \\ The Strand, London WC2R 2LS, United Kingdom \\ ${ }^{b}$ Perimeter Institute for Theoretical Physics, \\ Waterloo, Ontario, N2L 2Y5, Canada \\ E-mail: benjamin.assel@gmail.com, jgomis@perimeterinstitute.ca
}

ABSTRACT: Wilson loops in gauge theories pose a fundamental challenge for dualities. Wilson loops are labeled by a representation of the gauge group and should map under duality to loop operators labeled by the same data, yet generically, dual theories have completely different gauge groups. In this paper we resolve this conundrum for three dimensional mirror symmetry. We show that Wilson loops are exchanged under mirror symmetry with Vortex loop operators, whose microscopic definition in terms of a supersymmetric quantum mechanics coupled to the theory encode in a non-trivial way a representation of the original gauge group, despite that the gauge groups of mirror theories can be radically different. Our predictions for the mirror map, which we derive guided by branes in string theory, are confirmed by the computation of the exact expectation value of Wilson and Vortex loop operators on the three-sphere.

KEywords: Supersymmetry and Duality, Wilson, 't Hooft and Polyakov loops, Duality in Gauge Field Theories, Intersecting branes models

ArXiv EPrint: 1506.01718 


\section{Contents}

1 Introduction $\quad 2$

2 Loop operators in $3 \mathrm{~d} \mathcal{N}=4$ theories $\quad 6$

2.1 Wilson loop operators 9

$\begin{array}{lll}2.2 & \text { Vortex loop operators } & 10\end{array}$

3 Brane realization of loop operators and mirror map 13

$\begin{array}{lll}3.1 & \text { Brane realization of Wilson loop operators } & 16\end{array}$

3.2 Mirror of Wilson loops from S-duality 21

4 Mirror symmetry and loop operators: examples 28

$\begin{array}{lll}4.1 & T[\mathrm{SU}(2)] & 29\end{array}$

4.2 Wilson loops in the $\mathrm{U}(N-1)$ node of $T[\mathrm{SU}(N)] \quad 32$

4.3 The other $T[\mathrm{SU}(N)]$ loops $\quad 37$

4.4 Loops and mirror map in circular quivers 42

4.5 Loops in SQCD with $2 N$ quarks and its mirror 45

$5 \quad$ Loop operators in $3 \mathrm{~d} \mathcal{N}=4$ theories on $S^{3} \quad 48$

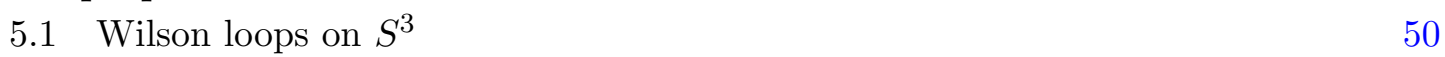

5.2 Vortex loops on $S^{3} \quad 51$

5.3 Exact partition function of 3d/1d theories on $S^{3}$

5.3.1 $S^{3}$ Partition function and Wilson loops $\quad 55$

5.3.2 $1 \mathrm{~d} \mathcal{N}=4$ Supersymmetric Quantum Mechanics partition function $\quad 57$

5.3.3 Matrix model computing Vortex loops $\quad 61$

5.4 Loops in $T[\mathrm{SU}(N)] \quad 64$

$\begin{array}{lll}5.4 .1 & T[\mathrm{SU}(2)] & 65\end{array}$

5.4.2 Wilson loops in $T[\mathrm{SU}(N)] \quad 66$

$\begin{array}{lll}5.4 .3 & \text { Vortex loops in } T[\mathrm{SU}(N)] & 67\end{array}$

$\begin{array}{lll}5.4 .4 & \text { Mirror map } & 71\end{array}$

$\begin{array}{lll}5.5 & \text { Loops in SQCD } & 73\end{array}$

$\begin{array}{lll}5.6 & \text { Hopping duality } & 75\end{array}$

A Supersymmetry transformations on $S^{3}$ and SQM embedding $\quad 80$

B Evaluations of the SQM index $\quad \mathbf{8 3}$

B.1 $\mathrm{U}(k)$ theory with $N$ fundamental and $M$ anti-fundamental chirals 83

B.2 $\mathrm{U}(k)$ theory with $N$ fundamental, $M$ anti-fundamental chirals and one adjoint chiral $\quad 84$

B.3 Two-node quiver 86 


\section{Introduction}

Wilson loop operators [1] play a central role in our understanding of gauge theories. A Wilson loop is specified by a curve $\gamma$ in spacetime and by the choice of a representation $\mathcal{R}$ of the gauge group $G$

$$
W_{\mathcal{R}}(\gamma)=\operatorname{Tr}_{R} P \exp \oint_{\gamma} i A_{\mu} d x^{\mu}
$$

In a certain sense, they are the most fundamental observables in gauge theories.

Wilson loops raise an immediate challenge to any conjectured duality, be it a field theory duality or a gauge/gravity duality: what is the dual description of Wilson loops? Even the more qualitative question of how the choice of a representation $\mathcal{R}$ of the gauge group $G$ labeling a Wilson is encoded in the dual theory is challenging, and in general the answer is unknown. Indeed, the gauge groups of theories participating in a field theory duality can be drastically different, and in gauge/gravity dualities there is not even a Lie group $G$ in sight in the dual bulk theory.

One may even argue that the existence of Wilson loops actually introduces a puzzle for dualities. While global symmetries and 't Hooft anomalies between dual theories must match, gauge symmetries between dual theories need not. In a sharp sense, gauge symmetries are not symmetries, but rather redundancies in our local description of particles of helicity one. Nevertheless, the gauge group $G$ is not void of important physical information about the theory: Wilson loop operators are labeled by a representation $\mathcal{R}$ of $G$. And therefore, it is in this vain, that the gauge group is "physical" and its elusive representations must be found in the dual.

In this paper we identify the dual description of half-supersymmetric Wilson loop operators in gauge theories related by three dimensional mirror symmetry [2], an infrared (IR) duality whereby two different ultraviolet (UV) $3 \mathrm{~d} \mathcal{N}=4$ gauge theories flow in the IR to the same superconformal field theory (SCFT):

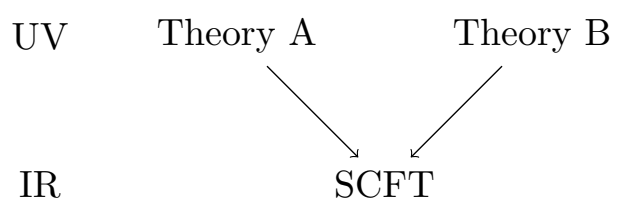

We find that there is a rather intricate "mirror map" relating Wilson loop operators in one theory to Vortex loop operators in the mirror:

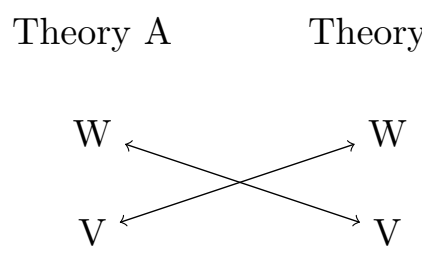

The mirror map in non-abelian gauge theories is rather subtle. In abelian gauge theories it follows from the mapping of the abelian global symmetries of the mirror dual theories [3-5]. ${ }^{1}$

\footnotetext{
${ }^{1}$ Vortex loop operators were previously studied in [6]. For Vortex loops in pure Chern-Simons theory see [7].
} 

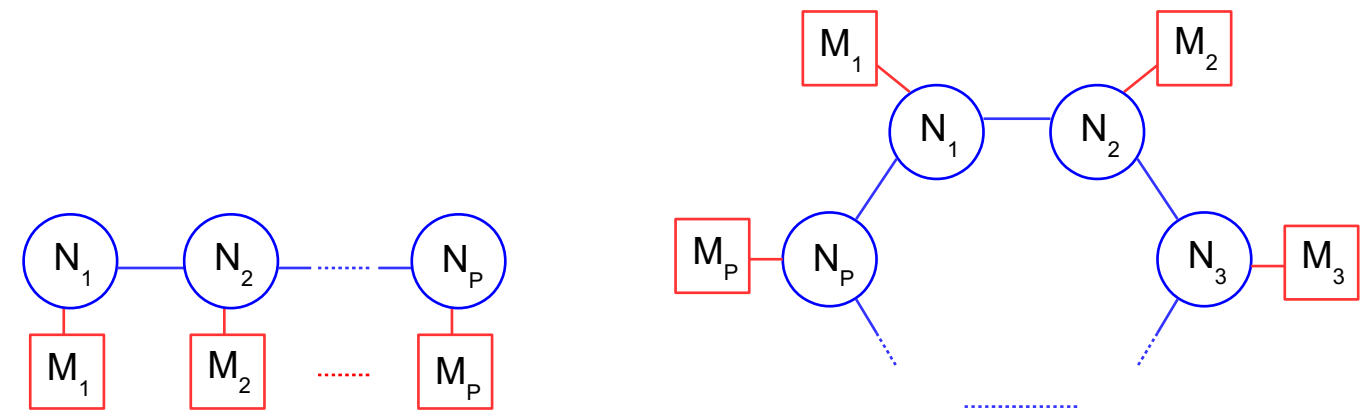

Figure 1. General linear and circular quiver diagrams.
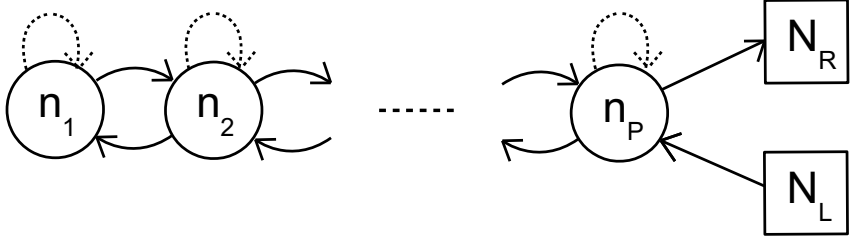

Figure 2. Dotted arrows reflect the possibility of adding an adjoint chiral multiplet in a gauge node.

We determine the mapping of loop operators under mirror symmetry for arbitrary $3 \mathrm{~d} \mathcal{N}=4$ gauge theories encoded by a quiver diagram of linear or circular topology (see figure 1).

We propose an explicit UV definition of the Vortex loop operators exchanged with Wilson loops under mirror symmetry. These Vortex loop operators are constructed by coupling $1 \mathrm{~d} \mathcal{N}=4$ supersymmetric quantum mechanics (SQM) quiver gauge theories supported on the loop to the bulk $3 \mathrm{~d} \mathcal{N}=4$ theory. The class of $1 \mathrm{~d} \mathcal{N}=4$ gauge theories that enter in the description of the mirror map of Wilson loop operators is encoded by the quiver diagram of figure $2 .^{2}$

Despite that the mirror dual $3 \mathrm{~d} \mathcal{N}=4$ gauge theories - denoted by Theory A and Theory B - typically have completely different gauge groups $G_{A}$ and $G_{B}$, we are able to encode the choice of representation $\mathcal{R}$ of a Wilson loop in one theory in the precise choice of the 1d quiver gauge theory in figure 2 describing the mirror dual Vortex loop operator. The $1 \mathrm{~d}$ quivers in this class are characterized by the ranks of their gauge nodes, the number of fundamental and anti-fundamental chirals in the last node and the presence or not of an adjoint chiral in each node. In particular, to a quiver gauge theory with all Fayet-Iliopoulos (FI) parameters $\eta_{i} \neq 0$, for $i=1, \ldots, p$, we associate a Wilson loop representation as follows: ${ }^{3}$

- $\mathcal{R}=\mathcal{S}_{n_{1}} \otimes \mathcal{S}_{n_{2}-n_{1}} \otimes \ldots \otimes \mathcal{S}_{n_{p}-n_{p-1}}$ : for a quiver with one adjoint chiral in each gauge node,

- $\mathcal{R}=\mathcal{A}_{n_{1}} \otimes \mathcal{A}_{n_{2}-n_{1}} \otimes \ldots \otimes \mathcal{A}_{n_{p}-n_{p-1}}$ : for a quiver with one adjoint chiral for $\mathrm{U}\left(n_{i}\right)$ for $i=1, \ldots, p-1$, but no adjoint chiral in the $\mathrm{U}\left(n_{p}\right)$ node,

\footnotetext{
${ }^{2}$ Arrows encode bifundamental chiral multiplets of two gauge nodes (circles) or of a gauge and a flavor node (square). For the details of superpotential couplings see section 2.2.

${ }^{3}$ See sections 3.1 and 5.3.2 for the general dictionary.
} 


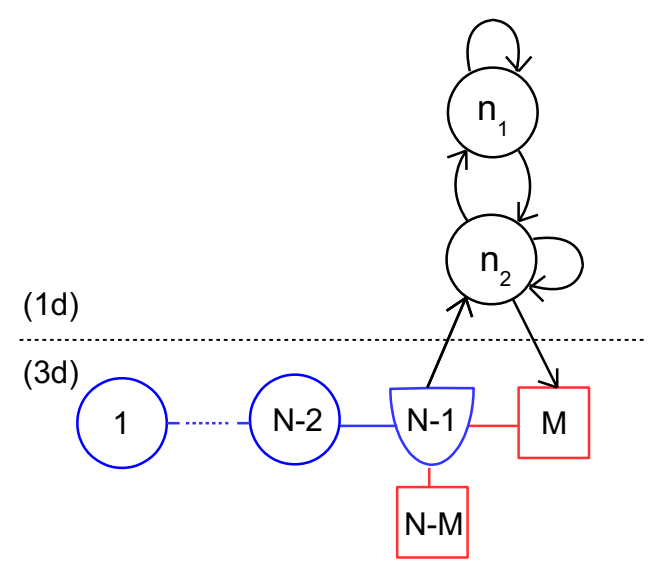

Figure 3. 3d/1d quiver realizing a Vortex loop in the $T[\mathrm{SU}(N)]$ theory.

where $\mathcal{A}_{k}$ and $\mathcal{S}_{k}$ denote the $k$-th antisymmetric and symmetric representations of $\mathrm{U}(N)_{L}$, if $\eta_{p}<0$, or $\mathrm{U}(N)_{R}$, if $\eta_{p}>0$. A generic product of symmetric and antisymmetric representations like $\mathcal{R}=\mathcal{S}_{n_{1}} \otimes \mathcal{A}_{n_{2}-n_{1}} \otimes \mathcal{A}_{n_{3}-n_{2}} \otimes \ldots \otimes \mathcal{S}_{n_{p}-n_{p-1}}$ is obtained by removing an adjoint chiral multiplet in some nodes. We propose, but have less quantitative evidence for, that an arbitrary irreducible representation $\mathcal{R}$ characterized by a Young diagram can be obtained from the family of quivers in figure 2 by setting all but one of the FI parameters to zero - that is $\eta_{p} \neq 0$-, i.e. $\eta_{1}=\eta_{2}=\ldots \eta_{p-1}=0{ }^{4}$

A Vortex loop associated to a given gauge group factor with $N$ fundamental hypermultiplets in the $3 \mathrm{~d} \mathcal{N}=4$ gauge theory is labeled by a representation $\mathcal{R}$ of the gauge group as well as by a splitting of $N$ into two factors: $N=M+(N-M)$. A Vortex loop labeled by a representation $\mathcal{R}$ and by the integer $M$ is constructed by gauging flavour symmetries of the $1 \mathrm{~d} \mathcal{N}=4$ quiver gauge theory with the bulk $3 \mathrm{~d} \mathcal{N}=4$ theory. The explicit $3 \mathrm{~d} / 1 \mathrm{~d}$ theory obtained in this way can be encoded by a mixed 3d/1d quiver diagram, see e.g. figure 3 .

The mirror map between loop operators that we uncover is rather intricate and rich. In particular, the map depends strongly on the choice of integer $M$ labeling a Vortex loop. As an illustrative example, the Vortex loop operator $V_{M, \mathcal{R}}$ in $T[\mathrm{SU}(N)]$ defined by the $3 \mathrm{~d} / 1 \mathrm{~d}$ quiver in figure 3 maps under mirror symmetry to the following combination of Wilson loop operators in $T[\mathrm{SU}(N)]$ (this theory is self-mirror) ${ }^{5}$

$$
\left\langle V_{M, \mathcal{R}}\right\rangle \stackrel{\text { mirror }}{\longleftrightarrow}\left\langle\sum_{s \in \Delta_{M}} W_{N, \mathbf{q}_{s}}^{\mathrm{fl}} W_{\widehat{\mathcal{R}}_{s}}^{\mathrm{U}(M)}\right\rangle \quad 0 \leq M \leq N-1,
$$

where $W_{\widehat{\mathcal{R}}_{s}}^{\mathrm{U}(M)}$ is a Wilson loop in a representation $\widehat{\mathcal{R}}_{s}$ of $\mathrm{U}(M), W_{N, \mathbf{q}_{s}}^{\mathrm{fl}}$ is an abelian flavour Wilson loop of charge $q_{s}$ and $\Delta_{M}$ is the set of representations that appear in the decomposition of $\mathcal{R}=\oplus_{s \in \Delta_{M}}\left(\mathbf{q}_{s}, \widehat{\mathcal{R}}_{s}\right)$ under the embedding $\mathrm{U}(1) \times \mathrm{U}(M) \subset \mathrm{U}(N-1) .{ }^{6}$ With the

\footnotetext{
${ }^{4}$ The same quiver but for $2 \mathrm{~d} \mathcal{N}=(2,2)$ gauge theories was shown to be labeled by an irreducible representation $\mathcal{R}$ in [8], where they describe M2-brane surface defects, which are indeed labeled by a representation $\mathcal{R}$.

${ }^{5}$ As we shall see in section 4.3 the Vortex loop operator $V_{N, \mathcal{R}}$ maps to a Wilson loop for the $\mathrm{U}(N-1) \subset$ $\mathrm{U}(N)$ flavour symmetry of $T[\mathrm{SU}(N)]$.

${ }^{6} \mathrm{U}(M)$ is embedded as $\mathrm{U}(M) \times \mathrm{U}(N-M-1) \subset \mathrm{U}(N-1)$, and the $\mathrm{U}(1)$ is the diagonal factor in $\mathrm{U}(N-M-1)$.
} 
algorithm we put forward in this paper, the mirror map between loop operators in arbitrary linear and circular quivers can be constructed, and we provide explicit representative examples for both types of quivers.

The key insight that allows us to construct the explicit mirror map between loop operators in linear and circular quivers is the identification of the brane realization of Wilson and Vortex loop operators in the Type IIB Hanany-Witten construction of $3 \mathrm{~d}$ $\mathcal{N}=4$ gauge theories [9]. ${ }^{7}$ In the string theory construction, mirror symmetry is realized as S-duality in Type IIB string theory [9]. By understanding the detailed physics of branes in string theory, the action of S-duality on our brane realization of loop operators allows us to find an explicit map between brane configurations, which in turn yields the mirror map between Wilson and Vortex loop operators.

We provide quantitative evidence for our mirror maps by computing the exact expectation value of circular Wilson and Vortex loop operators in $\mathcal{N}=4$ gauge theories on $S^{3}{ }^{8}$ The computation of the expectation value of Vortex loops combines in a rather interesting way the computations of the $S^{3}$ partition function in [17] with the supersymmetric quantum mechanics index of $[18,19]$. The detailed matrix integral capturing the expectation value is obtained by understanding how to couple the $1 \mathrm{~d} \mathcal{N}=4$ gauge theory defining the Vortex loop on an $S^{1} \subset S^{3}$ to the bulk gauge theory. All our computations confirm our brane-based predictions.

Our understanding of the action of duality on Wilson loops in the context of three dimensional mirror symmetry give us ideas and renewed confidence that this problem can also be tackled in other interesting dualities, like four dimensional Seiberg duality [20], where the gauge groups of the two dual theories are also different, and subject to the puzzles raised at the beginning. ${ }^{9}$

The plan of the rest of the paper is as follows. In section 2 we study the classes of loop operators that can be defined in a $3 \mathrm{~d} \mathcal{N}=4$ SCFT as well as a UV gauge theory definition of the SCFT. This analysis leads to consider Wilson and Vortex loop operators. In section 3, after reviewing the brane construction of $3 \mathrm{~d} \mathcal{N}=4$ gauge theories we put forward a brane realization of Wilson and Vortex loop operators. We give at least two different UV descriptions for each Vortex loop operator, distinguished in particular by the gauging of the global symmetries of the $1 \mathrm{~d}$ quiver gauge theory with bulk $3 \mathrm{~d}$ fields. The explicit $3 \mathrm{~d} / 1 \mathrm{~d}$ theories obtained this way can be encoded by mixed $3 \mathrm{~d} / 1 \mathrm{~d}$ quiver diagrams as in figure 4 . We then develop a brane-algorithm that allows us to use the action of S-duality on brane configurations to construct the mirror map for loop operators between dual gauge theories. This algorithm can be applied to an arbitrary $3 \mathrm{~d} \mathcal{N}=4$ gauge theory labeled by a linear or circular quiver. Section 4 presents the detailed mirror

\footnotetext{
${ }^{7}$ Our brane realization of loop operators in $3 \mathrm{~d} \mathcal{N}=4$ theories generalizes the brane realization of the defect field theory description of Wilson loop operators in $4 \mathrm{~d} \mathcal{N}=4$ super-Yang-Mills of [10, 11]. This brane construction was used in $[10,11]$ to provide the bulk holographic description of Wilson loops in an arbitrary representation of $\mathrm{SU}(N)$, following the dictionary put forward in $[12,13]$ for the fundamental representation.

${ }^{8}$ The mapping of the $S^{3}$ partition functions themselves between mirror dual theories was initiated in [14, $15]$ and proven in general for linear and circular unitary quiver theories in [16].

${ }^{9}$ Of course, in $4 \mathrm{~d} \mathcal{N}=1$ only null Wilson lines are supersymmetric.
} 

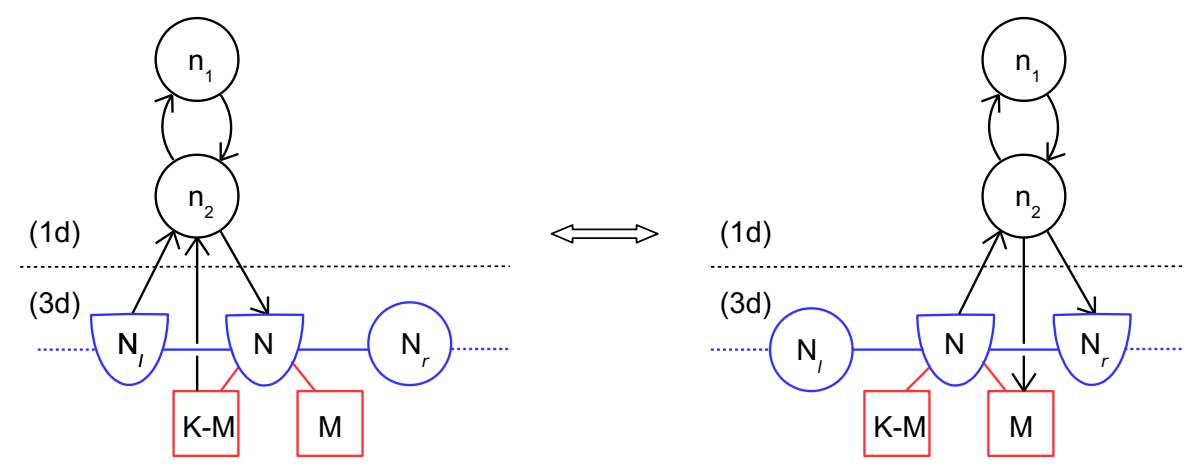

Figure 4. 3d/1d quiver theories realizing the same Vortex loop.

map for representative classes of gauge theories, including $T[\mathrm{SU}(N)]$, circular quivers with equal ranks on all nodes and supersymmetric QCD (SQCD). In section 5 we consider loop operators on $S^{3}$ and write down a matrix model representation of the exact expectation value of a Vortex loop operator in terms of the supersymmetric index of the SQM that defines it and the matrix model for the $3 \mathrm{~d}$ theory. We perform explicit computations of the expectation value of Wilson and Vortex loop operators and confirm our brane-based predictions for the mirror map. ${ }^{10}$ We also show that the distinct UV definitions of a given Vortex loop operator (see figure 4) give rise to the same operator in the IR, by showing that the expectation value of the two UV definitions coincide, and are related by hopping duality [21]. Some technical details are relegated to the appendices.

\section{Loop operators in $3 \mathrm{~d} \mathcal{N}=4$ theories}

An $\mathcal{N}=4$ SCFT is invariant under the $3 \mathrm{~d} \mathcal{N}=4$ superconformal symmetry $\operatorname{OSp}(4 \mid 4)$. The bosonic generators comprise those in the $\mathrm{SO}(3,2) \simeq \mathrm{Sp}(4)$ conformal algebra and those that generate the $\mathrm{SU}(2)_{C} \times \mathrm{SU}(2)_{H}$ R-symmetry of the SCFT. The supercharges in $\mathrm{OSp}(4 \mid 4)$ transform in the $(\mathbf{4 , 2}, \mathbf{2})$ representation of $\mathrm{SO}(3,2) \times \mathrm{SU}(2)_{C} \times \mathrm{SU}(2)_{H}$. A UV Lagrangian definition of the IR SCFT preserves $3 \mathrm{~d} \mathcal{N}=4$ Poincaré supersymmetry, the subgroup of $\operatorname{OSp}(4 \mid 4)$ that closes into the isometries of flat space. ${ }^{11}$ Mirror symmetry is the statement that a pair of UV gauge theories flow in the IR to the same SCFT with the roles of $\mathrm{SU}(2)_{C}$ and $\mathrm{SU}(2)_{H}$ exchanged:

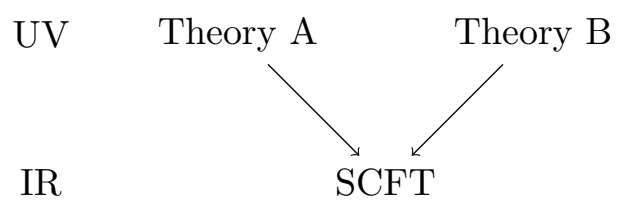

\footnotetext{
${ }^{10} \operatorname{In}[3,4]\langle V\rangle$ was computed using a disorder definition of $\mathrm{V}$ for abelian theories, which we reproduce from our SQM perspective and which we extend to arbitrary non-abelian gauge theories.

${ }^{11}$ The $3 \mathrm{~d} \mathcal{N}=4$ Poincaré supersymmetry algebra is the fixed locus of an involution of $\operatorname{OSp}(4 \mid 4)$.
} 
An $\mathcal{N}=4$ SCFT admits half-supersymmetric line operators/defects supported on a straight line in flat space. ${ }^{12}$ There are two physically inequivalent classes of superconformal line defects in an $\mathcal{N}=4$ SCFT, distinguished by their preserved symmetries. Superconformal line defects supported on a time-like line are invariant either under an $\mathrm{U}(1,1 \mid 2)_{W}$ or $\mathrm{U}(1,1 \mid 2)_{V}$ subalgebra of $\mathrm{OSp}(4 \mid 4) .{ }^{13}$ Let us exhibit this more explicitly. ${ }^{14}$ First, a straight time-like line in flat space preserves $\mathrm{SU}(1,1) \times \mathrm{U}(1)_{\perp} \subset$ $\mathrm{SO}(3,2)$. The $\mathrm{U}(1,1 \mid 2)_{W}$ subalgebra is embedded as follows. Under the embedding of $\mathrm{SU}(1,1) \times \mathrm{SU}(2)_{H} \times \mathrm{U}(1)_{\perp} \times \mathrm{U}(1)_{C}$ in $\mathrm{SO}(3,2) \times \mathrm{SU}(2)_{C} \times \mathrm{SU}(2)_{H}$, the supercharges generating $\operatorname{OSp}(4 \mid 4)$ decompose as $(\mathbf{2}, \mathbf{2})_{++} \oplus(\mathbf{2}, \mathbf{2})_{+_{-}} \oplus(\mathbf{2}, \mathbf{2})_{-+} \oplus(\mathbf{2}, \mathbf{2})_{--}$. The supercharges in $\mathrm{U}(1,1 \mid 2)_{W}$ are $(\mathbf{2}, \mathbf{2})_{++} \oplus(\mathbf{2}, \mathbf{2})_{--} .{ }^{15}$ For $\mathrm{U}(1,1 \mid 2)_{V}$ the analysis is identical with the roles of $\mathrm{SU}(2)_{C}$ and $\mathrm{SU}(2)_{H}$ exchanged. A defect preserving $\mathrm{U}(1,1 \mid 2)_{W}$ is invariant under $\mathrm{U}(1)_{C} \times \mathrm{SU}(2)_{H}$ while a defect preserving $\mathrm{U}(1,1 \mid 2)_{V}$ is invariant under $\mathrm{SU}(2)_{C} \times \mathrm{U}(1)_{H}$. The main goal of this paper is to give the UV description of these two classes of line operators/defects and to identity how the UV descriptions are mapped under mirror symmetry.

A line defect in a UV Lagrangian description of a SCFT can be made invariant under four of the supercharges in the $3 \mathrm{~d} \mathcal{N}=4$ Poincaré supersymmetry algebra of the UV theory. The $3 \mathrm{~d} \mathcal{N}=4$ Poincaré supercharges transform in the $(\mathbf{2}, \mathbf{2})$ representation of the $\mathrm{SU}(2)_{C} \times \mathrm{SU}(2)_{H}$ R-symmetry of the UV theory and obey

$$
\left\{Q_{\alpha A A^{\prime}}, Q_{\beta B B^{\prime}}\right\}=\left(\gamma_{\mu} C\right)_{\alpha \beta} P^{\mu} \epsilon_{A B} \epsilon_{A^{\prime} B^{\prime}} .
$$

We take the $\operatorname{SO}(2,1) \gamma$-matrices $\left(\gamma_{0}, \gamma_{1}, \gamma_{2}\right)=\left(i \tau_{3}, \tau_{1}, \tau_{2}\right)$, where $\tau_{a}$ are the Pauli matrices. The charge conjugation matrix is $C=\tau_{2}$. In Lorentzian signature and in this basis the reality condition on the supercharges is $Q_{\alpha A A^{\prime}}^{\dagger}=\left(\tau_{1}\right)_{\alpha}{ }_{\alpha} \epsilon^{A B} \epsilon^{A^{\prime} B^{\prime}} Q_{\beta B B^{\prime}}$.

There are two inequivalent $1 \mathrm{~d} \mathcal{N}=4$ supersymmetric quantum mechanics (SQM) subalgebras of the $3 \mathrm{~d} \mathcal{N}=4$ Poincaré superalgebra of a UV theory that can be preserved by a line defect. We denote them by $\mathrm{SQM}_{W}$ and $\mathrm{SQM}_{V} \cdot \mathrm{SQM}_{W}$ preserves $\mathrm{U}(1)_{C} \times \mathrm{SU}(2)_{H}$ and the supercharges $Q_{11 A^{\prime}}$ and $Q_{22 B^{\prime}}$, which anticommute to the generator of translations $H$ along the defect

$$
\left\{Q_{11 A^{\prime}}, Q_{22 B^{\prime}}\right\}=\epsilon_{A^{\prime} B^{\prime}} H .
$$

$\mathrm{SQM}_{V}$ preserves $\mathrm{SU}(2)_{V} \times \mathrm{U}(1)_{H}$ and the preserved supercharges $Q_{1 A 1}$ and $Q_{2 B 2}$ obey

$$
\left\{Q_{1 A 1}, Q_{2 B 2}\right\}=\epsilon_{A B} H .
$$

A $1 d \mathcal{N}=4 \mathrm{SQM}$ supersymmetry algebra can be brought to the canonical form

$$
\begin{aligned}
\left\{Q_{+} \bar{Q}_{+}\right\} & =H & {\left[J_{+}, Q_{+}\right] } & =-Q_{+} & {\left[J_{+}, \bar{Q}_{+}\right] } & =\bar{Q}_{+} \\
\left\{Q_{-}, \bar{Q}_{-}\right\} & =H & {\left[J_{-}, Q_{-}\right] } & =-Q_{-} & {\left[J_{-}, \bar{Q}_{-}\right] } & =\bar{Q}_{-}
\end{aligned}
$$

\footnotetext{
${ }^{12}$ Operators supported on curves obtained by acting on a straight line by broken conformal generators are also half-supersymmetric and preserve an isomorphic symmetry algebra. Under a broken conformal symmetry a time-like line becomes a rectangular time-like hyperbola, a space-like line becomes a space-like hyperbola (which includes a circle) and a null line remains a null line.

${ }^{13} \mathrm{U}(1,1 \mid 2)_{W}$ and $\mathrm{U}(1,1 \mid 2)_{V}$ are the fixed locus of an involution of $\mathrm{OSp}(4 \mid 4)$.

${ }^{14} \mathrm{~A}$ very similar analysis holds for a space-like line defect. For a null line defect, which we do not consider in this paper, the preserved symmetries are larger.

${ }^{15}$ The other choice $(\mathbf{2}, \mathbf{2})_{+-} \oplus(\mathbf{2}, \mathbf{2})_{-+}$corresponds to the same line defect but with opposite orientation.
} 
with all other (anti)-commutators vanishing. For both $\mathrm{SQM}_{W}$ and $\mathrm{SQM}_{V}$ algebras $Q_{+}=$ $Q_{111}$ and $\bar{Q}_{+}=Q_{222}=Q_{111}^{\dagger}$. For $\operatorname{SQM}_{W} Q_{-}=Q_{112}, \bar{Q}_{-}=-Q_{221}=Q_{112}^{\dagger}$ while for $\mathrm{SQM}_{V} Q_{-}=Q_{212}, \bar{Q}_{-}=-Q_{121}=Q_{212}^{\dagger}$. The embedding of the $\mathrm{SQM}_{W}$ and $\mathrm{SQM}_{V}$ algebras in the $3 \mathrm{~d} \mathcal{N}=4$ Poincaré superalgebra has a $\mathrm{U}(1)$ commutant: $R_{C}-J_{12}$ for $\mathrm{SQM}_{W}$ and $R_{H}-J_{12}$ for $\mathrm{SQM}_{V}$, where $J_{12}$ is the $\mathrm{U}(1)_{\perp}$ rotation generator transverse to the defect and $R_{C}$ and $R_{H}$ are the Cartan generators of $\mathrm{SU}(2)_{C}$ and $\mathrm{SU}(2)_{H}$ respectively. ${ }^{16}$ Therefore, up to shifts by the "flavour" symmetry $R_{C}-J_{12}$ for $\mathrm{SQM}_{W}$ and by $R_{H}-J_{12}$ for $\mathrm{SQM}_{V}$ the Cartan R-symmetry generators can be taken to be $J_{+}=-R_{C}-R_{H}$ and $J_{-}=R_{H}-R_{C}$.

In summary, in a $3 \mathrm{~d} \mathcal{N}=4$ gauge theory there are two classes of line defects that can defined, one preserving $\mathrm{SQM}_{W}$ and the other $\mathrm{SQM}_{V}$. These line defects flow in the IR to superconformal line defects preserving $\mathrm{U}(1,1 \mid 2)_{W}$ and $\mathrm{U}(1,1 \mid 2)_{V}$ respectively. Our analysis can be succinctly summarized by the following diagram:

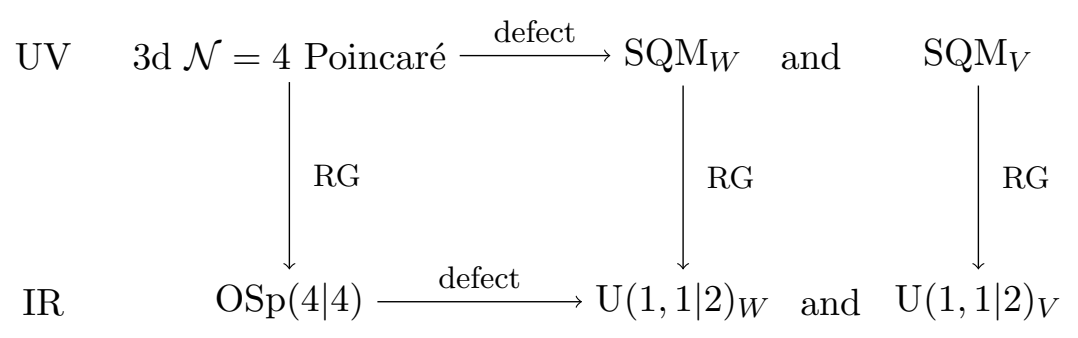

Both classes of UV supersymmetric line defects in a UV $3 \mathrm{~d} \mathcal{N}=4$ theory can be realized by coupling different $1 \mathrm{~d} \mathcal{N}=4 \mathrm{SQM}$ theories with four supercharges to the bulk $3 \mathrm{~d} \mathcal{N}=4$ theory. A canonical way of coupling a $1 \mathrm{~d} \mathcal{N}=4 \mathrm{SQM}$ theory to a $3 \mathrm{~d} \mathcal{N}=$ 4 theory is by gauging flavour symmetries of the SQM theory with $3 \mathrm{~d} \mathcal{N}=4$ vector multiplets. The gauging of the defect flavour symmetries with bulk vector multiplets is made supersymmetric by embedding the defect vector multiplet of the supersymmetry algebra preserved by the defect into the bulk $3 \mathrm{~d} \mathcal{N}=4$ vector multiplet at the position of the line defect. The embedding is found by identifying which combination of fields in the higher dimensional vector multiplet transform as the fields of the defect vector multiplet under the supersymmetry preserved by the defect. Replacing the defect vector multiplet fields in the gauged $1 \mathrm{~d} \mathcal{N}=4$ SQM theory with the proper combination of bulk vector multiplet fields ensures that the coupling of $1 \mathrm{~d}$ fields to $3 \mathrm{~d}$ fields is supersymmetric under the supersymmetry of the defect $1 \mathrm{~d} \mathcal{N}=4$ SQM theory. Superpotential couplings between defect and bulk matter multiplets may also be added when defect matter multiplets can be embedded in bulk hypermultiplets. Such couplings gauge defect flavour symmetries with bulk flavour or gauge symmetries, depending on which symmetries of the bulk matter multiplet are global and which are gauged.

We consider UV line defects invariant under $\mathrm{SQM}_{W}$ and $\mathrm{SQM}_{V}$ obtained by gauging global symmetries of $1 \mathrm{~d} \mathcal{N}=4 \mathrm{SQM}$ theories obtained by dimensional reduction of $2 \mathrm{~d}$

\footnotetext{
${ }^{16} R_{C}-J_{12}$ and $R_{H}-J_{12}$ is also the commutant of $\mathrm{U}(1,1 \mid 2)_{W}$ and $\mathrm{U}(1,1 \mid 2)_{V}$ in $\mathrm{OSp}(4 \mid 4)$. They appear respectively in the anticommutator of the $\mathrm{U}(1,1 \mid 2)_{W}$ and $\mathrm{U}(1,1 \mid 2)_{V}$ supercharges preserved by the corresponding defect.
} 
$\mathcal{N}=(0,4)$ and $2 \mathrm{~d} \mathcal{N}=(2,2)$ theories with $3 \mathrm{~d} \mathcal{N}=4$ vector multiplets:

- $\mathrm{SQM}_{W}: 2 \mathrm{~d} \mathcal{N}=(0,4) \rightarrow 1 \mathrm{~d} \mathcal{N}=4 \mathrm{SQM}$

- $\mathrm{SQM}_{V}: 2 \mathrm{~d} \mathcal{N}=(2,2) \rightarrow 1 \mathrm{~d} \mathcal{N}=4 \mathrm{SQM}$

Superpotential couplings between defect and bulk fields also play an important role in the construction of defects.

\subsection{Wilson loop operators}

A line defect in a UV $3 \mathrm{~d} \mathcal{N}=4$ gauge theory invariant under $\mathrm{SQM}_{W}$ is the Wilson line operator, which is labeled by a representation $\mathcal{R}$ of the gauge group. It is given by

$$
W_{\mathcal{R}}=\operatorname{Tr}_{\mathcal{R}} P \exp \oint i\left(A_{\mu} \dot{x}^{\mu}+\sqrt{-\dot{x}^{2}} \sigma\right) d \tau
$$

where $\sigma \equiv \sigma_{3}$ is the scalar field in the $\mathcal{N}=2$ vector multiplet inside the $\mathcal{N}=4$ vector multiplet. This operator manifestly breaks the $\mathrm{SU}(2)_{C}$ symmetry acting on the three scalars $\vec{\sigma}=\left(\sigma_{1}, \sigma_{2}, \sigma_{3}\right)$ in the $\mathcal{N}=4$ vector multiplet down to $\mathrm{U}(1)_{C},{ }^{17}$ while preserving $\mathrm{SU}(2)_{H}$. If the operator is supported on a straight line, ${ }^{18}$ it preserves the $1 \mathrm{~d} \mathcal{N}=4$ SQM subalgebra $\mathrm{SQM}_{W}$ of the $3 \mathrm{~d} \mathcal{N}=4$ theory and its $\mathrm{U}(1)_{C} \times \mathrm{SU}(2)_{H}$ R-symmetry. In the IR a Wilson line operator flows to a conformal line operator in the SCFT preserving $\mathrm{U}(1,1 \mid 2)_{W}$.

The supersymmetric Wilson line operator (2.5) can be realized by coupling a $1 \mathrm{~d} \mathcal{N}=4$ SQM theory living on the line with the bulk $3 \mathrm{~d} \mathcal{N}=4$ gauge theory. This coupling preserves the $\mathrm{SQM}_{W}$ supersymmetry algebra. The defect $1 \mathrm{~d} \mathcal{N}=4 \mathrm{SQM}$ that represents a Wilson loop operator is the theory of a $1 \mathrm{~d} \mathcal{N}=4$ fermi multiplet, obtained by dimensional reduction of the $2 \mathrm{~d} \mathcal{N}=(0,4)$ fermi multiplet, which on-shell consists of a complex chiral fermion. The flavour symmetry of a $1 \mathrm{~d} \mathcal{N}=(0,4)$ fermi multiplet can be gauged preserving supersymmetry with a $1 \mathrm{~d} \mathcal{N}=(0,4)$ vector multiplet. The fields of the $1 \mathrm{~d} \mathcal{N}=(0,4)$ vector multiplet that couple to the fermi multiplet can be embedded in the $3 \mathrm{~d} \mathcal{N}=4$ vector multiplet as follows ${ }^{19}$

$$
\begin{aligned}
a_{0} & =A_{0} \\
\sigma_{1 d} & =\sigma .
\end{aligned}
$$

\footnotetext{
${ }^{17}$ The choice of scalar determines an embedding of $\mathrm{U}(1)_{C}$ in $\mathrm{SU}(2)_{C}$.

${ }^{18}$ When the scalar couples to the loop with constant charge a circular Wilson loop is not supersymmetric in the UV theory. See, however, discussion at the end of this subsection and of circular Wilson loops on $S^{3}$ in section 5.1.

${ }^{19}$ The Fermi multiplet couples only to a subset of fields in the $\mathcal{N}=(0,4)$ vector multiplet, and those do admit an embedding into the $3 \mathrm{~d} \mathcal{N}=4$ vector multiplet. This observation can be applied to the study of supersymmetric surface operators in $4 \mathrm{~d} \mathcal{N}=2$, which can preserve either $2 \mathrm{~d} \mathcal{N}=(2,2)$ or $\mathcal{N}=(0,4)$ supersymmetry. We can construct a surface operator by gauging a $\mathcal{N}=(0,4)$ fermi multiplet with a bulk vector multiplet. These surface operators were studied in the context of $\mathcal{N}=4 \mathrm{SYM}$ in [22].
} 
This embedding makes manifest that the coupling of the fermi multiplet to the bulk $\mathcal{N}=4$ vector multiplet preserves $\mathrm{U}(1)_{C} \times \mathrm{SU}(2)_{H}$ R-symmetry and the $\mathrm{SQM}_{W}$ algebra. ${ }^{20}$

The $1 \mathrm{~d} \mathcal{N}=4 \mathrm{SQM}$ fermi multiplet on the defect can be integrated out exactly and it results in the insertion of a supersymmetric Wilson loop (2.5) in the bulk $3 \mathrm{~d} \mathcal{N}=4$ theory. This representation of a supersymmetric Wilson loop in $4 \mathrm{~d} \mathcal{N}=4$ super-Yang-Mills (SYM) as a coupling of a fermi multiplet with bulk fields appeared in $[10,11]$, where the defect field theory was derived from brane intersections in string theory. Inspired by [10, 11], a brane realization of Wilson loop defects in $3 \mathrm{~d} \mathcal{N}=4$ gauge theories will play a prominent role in section 3, where we will use S-duality of Type IIB string theory to identify the mirror of Wilson loop operators.

As an aside, 1/4-supersymmetric Wilson loops supported on an arbitrary curve $\gamma$ in $R^{3}$ can be defined mimicking the construction in [23] of 1/16-supersymmetric Wilson loops in $4 \mathrm{~d} \mathcal{N}=4 \mathrm{SYM}$. This requires tuning the coupling of the loop to the three scalars in the vector multiplet. Explicitly, 1/4-supersymmetric Wilson loops are given by

$$
\operatorname{Tr}_{\mathcal{R}} P \exp \oint_{\gamma} i\left(A_{\mu}+i \sigma_{\mu}\right) \dot{x}^{\mu} d \tau
$$

and preserve two supercharges: $Q_{A^{\prime}}^{H} \equiv Q_{\alpha A A^{\prime}} \epsilon^{\alpha A}$. These Wilson loop operators are in the cohomology of the supercharges $Q_{A^{\prime}}^{H}$ of the Rozansky-Witten twisted theory [24] $]^{21}$ obtained by twisting spatial rotations with $\mathrm{SU}(2)_{C}$. Half-supersymmetric Higgs branch operators are also in the cohomology of this twisted theory.

\section{$2.2 \quad$ Vortex loop operators}

A supersymmetric line defect in a UV $3 \mathrm{~d} \mathcal{N}=4$ theory preserving $\mathrm{SQM}_{V}$ can be constructed by coupling the bulk theory to a $1 \mathrm{~d} \mathcal{N}=4 \mathrm{SQM}$ theory with $\mathrm{SQM}_{V}$ symmetry. For line defects preserving $\mathrm{SQM}_{V}$, the appropriate $1 \mathrm{~d} \mathcal{N}=4 \mathrm{SQM}$ theories are obtained by dimensionally reducing $4 \mathrm{~d} \mathcal{N}=1$ theories (or equivalently $2 \mathrm{~d} \mathcal{N}=(2,2)$ theories). $\mathrm{U}(1)_{H}$ is the R-symmetry already present in $4 \mathrm{~d}$ while $\mathrm{SU}(2)_{V}$ emerges as an R-symmetry in the dimensional reduction down to $1 \mathrm{~d}$. Therefore the $\mathrm{SQM}_{V}$ invariant $1 \mathrm{~d} \mathcal{N}=4 \mathrm{SQM}$ theories we consider are supersymmetric gauge theories based on the familiar $4 \mathrm{~d} \mathcal{N}=1$ vector multiplets and chiral multiplets. The same $4 \mathrm{~d} \mathcal{N}=1$ theories dimensionally reduced to $2 \mathrm{~d}$ define surface operators [26] in $4 \mathrm{~d} \mathcal{N}=2$ gauge theories.

We can construct a supersymmetric line defect in a $3 \mathrm{~d} \mathcal{N}=4$ gauge theory by gauging flavour symmetries of a $1 \mathrm{~d} \mathcal{N}=4 \mathrm{SQM}_{V}$ invariant theory with bulk vector multiplets. The embedding of the bosonic fields in the $1 \mathrm{~d}$ vector multiplet $\left(a_{3}, \vec{\sigma}_{1 d}, d\right)$, where $\vec{\sigma}_{1 d}$ is a triplet of $\mathrm{SU}(2)_{V}$, in the $3 \mathrm{~d} \mathcal{N}=4$ vector multiplet is (see appendix A)

$$
\begin{aligned}
a_{0} & =A_{0} \\
\vec{\sigma}_{1 d} & =\vec{\sigma} \\
d & =D+F_{12} .
\end{aligned}
$$

\footnotetext{
${ }^{20}$ The purely $1 \mathrm{~d} \mathcal{N}=4$ theory of a gauged fermi multiplet is invariant under $\mathrm{SO}(4) \mathrm{R}$-symmetry. The coupling of the fermi multiplet with the bulk through the embedding (2.7) breaks the R-symmetry down to $\mathrm{U}(1)_{C} \times \mathrm{SU}(2)_{H}$.

${ }^{21}$ This is the $3 \mathrm{~d}$ counterpart of the statement in $4 \mathrm{~d} \mathcal{N}=4 \mathrm{SYM}$ that the $1 / 16$-supersymmetric Wilson loop operators in [23] are in the cohomology of a supercharge of the Langlands twist [25].
} 
This embedding makes manifest that $\mathrm{SU}(2)_{V}$ is preserved and that $\mathrm{SU}(2)_{H}$ is broken down to $\mathrm{U}(1)_{H}$, as it selects one of the auxiliary fields transforming as a triplet of $\mathrm{SU}(2)_{H}$ in the $3 \mathrm{~d} \mathcal{N}=4$ vector multiplet, which we have denoted by $D .{ }^{22}$ The coupled theory preserves the $\mathrm{SQM}_{V}$ algebra.

We can gauge defect flavour symmetries either with $3 \mathrm{~d} \mathcal{N}=4$ fluctuating vector multiplets or background vector multiplets. Background vector multiplets for flavour symmetries are associated to canonical supersymmetric mass deformations in $3 \mathrm{~d} \mathcal{N}=4$ and $1 \mathrm{~d} \mathcal{N}=4$ theories. ${ }^{23}$ Gauging 1d flavour symmetries with background 3d vector multiplets means that $1 \mathrm{~d}$ and $3 \mathrm{~d}$ flavour symmetries are identified. $1 \mathrm{~d} \mathcal{N}=4$ and $3 \mathrm{~d} \mathcal{N}=4$ flavour symmetries are identified by $\mathrm{SQM}_{V}$-preserving defect cubic superpotential couplings between defect chiral multiplets and bulk hypermultiplets ${ }^{24}$

$$
W=\tilde{q}_{I}^{i} q_{a}^{I} Q_{i}^{a},
$$

where the index $I$ is a $1 \mathrm{~d}$ gauge index. The indices $i, a$ are simultaneously indices for $1 \mathrm{~d}$ flavour symmetries and indices for either $3 \mathrm{~d}$ flavour or gauge symmetries. When $a$ (or $i$ ) is a $3 \mathrm{~d}$ flavour index, the superpotential breaks the (otherwise independent) flavour symmetries acting on chiral multiplets $q_{a}$ (or $\tilde{q}^{i}$ ) and hypermultiplets $Q_{i}^{a}$ to the diagonal flavour symmetry group. ${ }^{25}$ The background $3 \mathrm{~d} \mathcal{N}=4$ vector multiplet gives the same mass to the $1 \mathrm{~d}$ chiral multiplets and $3 \mathrm{~d}$ hypermultiplets that are acted on by the preserved diagonal flavour symmetry group.

The $1 \mathrm{~d} \mathcal{N}=4$ gauge theories that appear in the construction of the defects dual to Wilson loops can be encoded in a standard quiver diagram shown in figure $2 .^{26}$

An adjoint chiral multiplet may be added to any $\mathrm{U}\left(n_{i}\right)$ gauge group factor, an option which we denote by a dashed line. Each adjoint chiral multiplet is coupled to the neighbouring bifundamental chiral multiplets through a cubic superpotential, while nodes without an adjoint chiral multiplet have an associated quartic superpotential coupling the corresponding bifundamental chiral multiplets.

The specific couplings between $1 \mathrm{~d} \mathcal{N}=4$ and $3 \mathrm{~d} \mathcal{N}=4$ theories can be encoded in a combined $3 \mathrm{~d} / 1 \mathrm{~d}$ quiver diagram (analogous $4 \mathrm{~d} / 2 \mathrm{~d}$ quivers have appeared in [21] (see also [8])). The quiver diagram makes explicit the $1 \mathrm{~d}$ flavour symmetries which are gauged with bulk dynamical gauge fields and the flavour symmetries which are identified with $3 \mathrm{~d}$ flavour symmetries, as shown in figure 5 . We use the mixed circle and square notation of [8] to denote the $1 \mathrm{~d}$ flavour symmetries that are gauged with dynamical $3 \mathrm{~d}$ vector multiplets. This $3 \mathrm{~d} / 1 \mathrm{~d}$ quiver also assigns a defect cubic superpotential coupling between $1 \mathrm{~d}$ chiral multiplets and $3 \mathrm{~d}$ hypermultiplets for each triangle that can be formed with these fields.

\footnotetext{
${ }^{22}$ The choice of auxiliary field determines an embedding of $\mathrm{U}(1)_{H}$ in $\mathrm{SU}(2)_{H}$.

${ }^{23}$ Obtained by turning on constant commuting values for the three scalars in the $1 \mathrm{~d}$ and $3 \mathrm{~d} \mathcal{N}=4$ vector multiplet.

${ }^{24}$ The fields in the $1 \mathrm{~d} \mathcal{N}=4$ chiral multiplet can be embedded in the $3 \mathrm{~d} \mathcal{N}=4$ hypermultiplet. This embedding (see appendix A), which we denote by $Q$, allows one to write supersymmetric couplings between defect chiral multiplets and bulk hypermultiplets.

${ }^{25}$ When one of the indices is a $3 \mathrm{~d}$ gauge index the superpotential indeed enforces the gauging of $1 \mathrm{~d}$ flavour symmetries with a $3 \mathrm{~d}$ dynamical vector multiplet.

${ }^{26}$ These quivers but in a $2 \mathrm{~d} \mathcal{N}=(2,2)$ were used in [8] to describe M2-brane surface operators.
} 


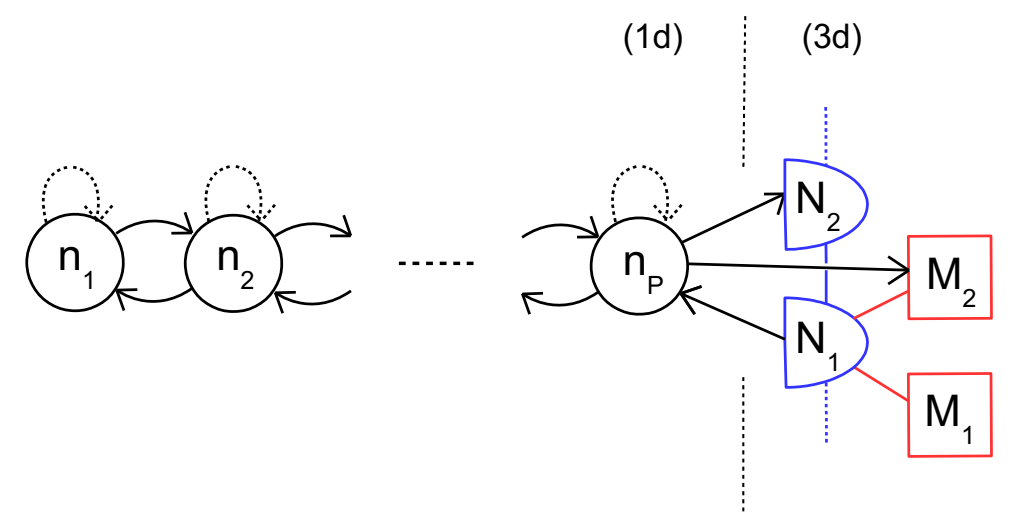

Figure 5. 1d quiver theory coupled to $3 \mathrm{~d}$ quiver theory by gauging $1 \mathrm{~d}$ flavor symmetries with $3 \mathrm{~d}$ vector mutiplets (dynamical or weakly gauged).

Demanding that the UV supersymmetric $3 \mathrm{~d} / 1 \mathrm{~d}$ Lagrangian coupling $1 \mathrm{~d}$ chiral multiplets with a $3 \mathrm{~d} \mathcal{N}=4$ vector multiplet is well-defined (finite) requires that [26-28]

$$
F_{12}=g^{2} \mu \delta^{2}(x),
$$

where $\mu$ is the moment map for the flavour symmetry acting on the $1 \mathrm{~d}$ chiral multiplets that is gauged with the bulk (dynamical) vector multiplet and $g$ is its $3 \mathrm{~d}$ gauge coupling. Therefore, in the semiclassical UV description, defect fields induce a singular Vortex field configuration on the $3 \mathrm{~d}$ gauge fields. This justifies our use of the subscript $V$ to describe this class of line defects, which we refer as Vortex line defects/operators. These UV Vortex line defects flow in the IR to conformal line operators in the SCFT preserving $\mathrm{U}(1,1 \mid 2)_{V}$.

As another aside, we note that just as a 1/4-supersymmetric Wilson loop supported on an arbitrary curve $\gamma$ in $R^{3}$ have been constructed in (2.8), it should be possible to construct a 1/4-supersymmetric Vortex loop on an arbitrary curve in $R^{3}$ by suitably adjusting the coupling of the $1 \mathrm{~d} \mathcal{N}=4 \mathrm{SQM}$ to the bulk $3 \mathrm{~d} \mathcal{N}=4$ theory. Such a Vortex loop would preserve two supercharges: $Q_{A}^{C} \equiv Q_{\alpha A A^{\prime}} \epsilon^{\alpha A^{\prime}}$. These Vortex loop operators are in the cohomology of the supercharges $Q_{A}^{C}$ of the other version of the Rozansky-Witten twisted theory, obtained by twisting spatial rotations with $\mathrm{SU}(2)_{H}$. Half-supersymmetric Coulomb branch operators, that is monopole operators, are also in the cohomology of this twisted theory.

Given two UV mirror theories that flow in the IR to the same SCFT, we can construct both classes of line operators in each of the UV theories. How are line operators mapped under mirror symmetry? Since mirror symmetry exchanges $\mathrm{SU}(2)_{C}$ with $\mathrm{SU}(2)_{H}$ in dual mirror theories, Wilson line operators of one theory are mapped to Vortex line operators in the mirror and viceversa. This can be represented by the following diagram:

\section{Theory A Theory B}

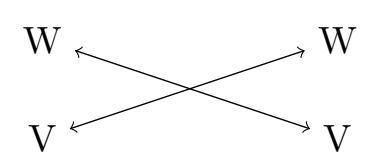




\begin{tabular}{|c|c|c|c|c|c|c|c|c|c|c|}
\hline & 0 & 1 & 2 & 3 & 4 & 5 & 6 & 7 & 8 & 9 \\
\hline D3 & $\mathrm{X}$ & $\mathrm{X}$ & $\mathrm{X}$ & $\mathrm{X}$ & & & & & & \\
D5 & $\mathrm{X}$ & $\mathrm{X}$ & $\mathrm{X}$ & & $\mathrm{X}$ & $\mathrm{X}$ & $\mathrm{X}$ & & & \\
NS5 & $\mathrm{X}$ & $\mathrm{X}$ & $\mathrm{X}$ & & & & & $\mathrm{X}$ & $\mathrm{X}$ & $\mathrm{X}$ \\
\hline
\end{tabular}

Table 1. Brane array for three-dimensional quiver gauge theories.

Our immediate goal is to come up with an algorithm that yields the duality map between Wilson and Vortex loop operators in mirror dual theories.

\section{Brane realization of loop operators and mirror map}

In this section we first briefly introduce the Type IIB string theory realization of $3 \mathrm{~d} \mathcal{N}=4$ gauge theories of [9] and recall how mirror symmetry gets realized as S-duality in string theory. Central to the main goal of this paper is the brane realization of both types of line defects discussed in the previous section that we put forward in this section. We then devise an explicit algorithm using branes in string theory to identify the map between loop operators in mirror dual theories.

$3 \mathrm{~d} \mathcal{N}=4$ supersymmetric gauge theories admit an elegant realization as the lowenergy limit of brane configurations in Type IIB string theory [9]. This consists of an array of D3, D5 and NS5 branes oriented as shown in table $1 .{ }^{27}$

The gauge theory associated to a brane configuration is constructed by assigning:

- A $\mathrm{U}(N)$ vector multiplet to N D3-branes suspended between two NS5-branes

- A hypermultiplet in the fundamental representation of $\mathrm{U}(N)$ to a D5-brane intersecting N D3-branes stretched between two NS5-branes

- A hypermultiplet in the bifundamental representation of $\mathrm{U}\left(N_{1}\right) \times \mathrm{U}\left(N_{2}\right)$ to an NS5brane with $N_{1}$ D3-branes ending on its left and $N_{2}$ branes ending on its right

Depending on whether the $x^{3}$ coordinate takes values on the line or is circle valued, the $3 \mathrm{~d} \mathcal{N}=4$ gauge theories engineered this way are described either by quiver diagrams of linear topology or circular topology: linear and circular quiver diagrams respectively. The quiver diagrams for linear and circular quiver theories are presented in figure 1 . The general brane configuration realizing a linear quiver theory is shown in figure 6. For a circular quiver the $x^{3}$ direction is periodic and there are extra D3-branes stretched between the first and last NS5-branes. ${ }^{28}$

We are interested in $3 \mathrm{~d} \mathcal{N}=4$ gauge theories that flow in the IR to an irreducible, interacting SCFT. A $3 \mathrm{~d} \mathcal{N}=4$ gauge theory flows to such a SCFT in the IR if each gauge

\footnotetext{
${ }^{27}$ For more details of these brane constructions see [9, 29-31].

${ }^{28}$ The number inside a circle denotes the rank of a gauge group factor. The number inside a rectangle denotes the number of hypermultiplets in the fundamental representation of the gauge group factor corresponding to the circle to which attaches. A line between two circles represents a bifundamental hypermultiplet of the two gauge group factors connected by the line.
} 


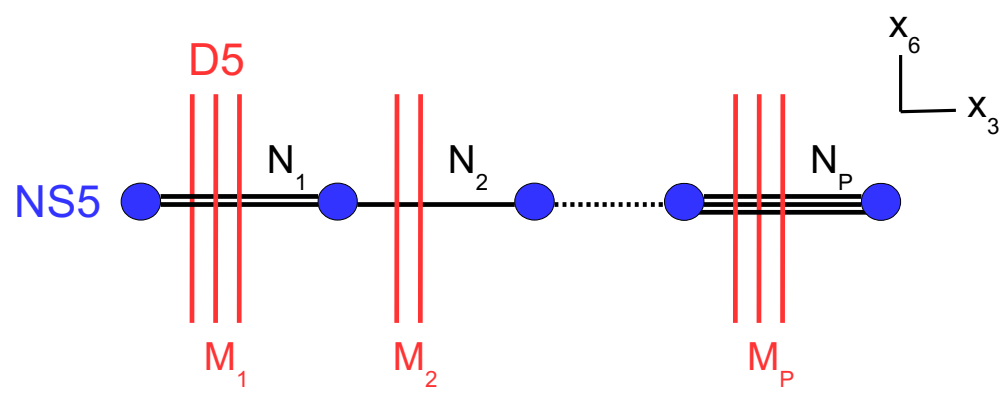

Figure 6. Brane configuration realizing a linear quiver theory.

group factor $\mathrm{U}\left(N_{c}\right)$ has a number of fundamental hypermultiplets $N_{f}$ obeying $N_{f} \geq 2 N_{c}$ and $\sum_{i} M_{i} \geq 2$. The second condition is automatically obeyed by linear quivers obeying the first condition, but it is an extra requirement for circular quivers. When these conditions are satisfied the gauge group of the quiver can be completely Higgsed [29, 32] and there are no monopole operators hitting the unitarity bound [29]. ${ }^{29}$ Mirror symmetry is the statement that two different $3 \mathrm{~d} \mathcal{N}=4$ gauge theories flow in the IR to the same irreducible SCFT.

The $\mathrm{SU}(2)_{C} \times \mathrm{SU}(2)_{H}$ R-symmetry of such an irreducible SCFT coincides with the $\mathrm{SU}(2)_{C} \times \mathrm{SU}(2)_{H} \mathrm{R}$-symmetry of the UV gauge theory. In the brane construction the Rsymmetry is realized geometrically as spacetime rotations: $\mathrm{SU}(2)_{C}$ rotates $x^{789}$ and $\mathrm{SU}(2)_{H}$ rotates $x^{456}$. 3d $\mathcal{N}=4$ gauge theories admit moduli spaces of vacua: the Coulomb branch and the Higgs branch. ${ }^{30}$ These are hyperkähler manifolds invariant under $\mathrm{SU}(2)_{H}$ and $\mathrm{SU}(2)_{C}$ and acted on by a group of isometries $G_{C}$ and $G_{H}$ respectively. $G_{H}$ is manifest in the UV definition of the SCFT and is realized as the flavour symmetry acting on the hypermultiplets, while only the Cartan subalgebra of $G_{C}$ is manifest in the UV. Each U(1) gauge group factor gives rise to a manifest U(1) global symmetry, known as a topological symmetry, which acts on the Coulomb branch. The abelian symmetry acting on the Coulomb branch can be enhanced to a non-abelian $G_{C}$ symmetry when conserved currents associated to the roots of $G_{C}$ can be constructed with monopoles operators. ${ }^{31}$ The nontrivial, irreducible SCFT sits at the intersection of the Higgs and Coulomb branch where the R-symmetry is enhanced to $\mathrm{SU}(2)_{C} \times \mathrm{SU}(2)_{H}$. The IR SCFT inherits a $G_{C} \times G_{H}$ global symmetry. In the brane realization, the Coulomb branch corresponds to the motion of D3-branes along $x^{789}$ while the Higgs branch to the motion of D3-branes along $x^{456} .32$

The brane description of $3 \mathrm{~d} \mathcal{N}=4 \mathrm{UV}$ gauge theories gives an elegant realization of mirror symmetry [9], whereby two different UV gauge theories flow to the same nontrivial SCFT in the IR with the roles of $\mathrm{SU}(2)_{C}$ and $\mathrm{SU}(2)_{H}$ exchanged. Mirror symmetry is realized as S-duality in Type IIB string theory combined with a spacetime rotation that

\footnotetext{
${ }^{29}$ When $N_{f}<2 N_{c}$ or $\sum_{i=1}^{\hat{P}} M_{i} \leq 2$ for circular quivers the IR theory is believed to contain a decoupled sector.

${ }^{30}$ Mixed branches can emerge at submanifolds of the Higgs and Coulomb branch.

${ }^{31} G_{C}$ maps to the flavour symmetry acting on the hypermultiplets of the mirror theory.

${ }^{32}$ The brane realization makes it clear why $\sum_{i=1}^{\hat{P}} M_{i} \geq 2$ is required for complete Higgsing in circular quivers. Indeed, unless there are two D5-branes, D3-branes segments cannot be detached from the NS5-branes.
} 
a)

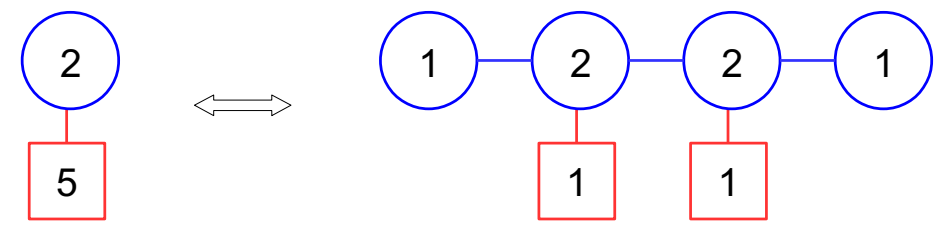

b)

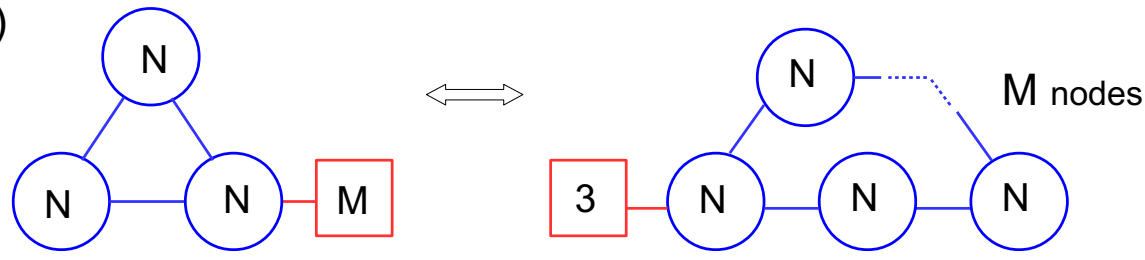

Figure 7. a) A pair of mirror dual linear quivers. b) A pair of mirror dual circular quivers.

sends $x^{456}$ to $x^{789}$ and $x^{789}$ to $-x^{456}$, which exchanges $\mathrm{SU}(2)_{C}$ with $\mathrm{SU}(2)_{H}$. The combined transformation, which we will refer as S-duality for brevity, maps the class of brane configurations we have discussed to itself. Given the brane configuration corresponding to a UV $3 \mathrm{~d} \mathcal{N}=4$ gauge theory, the mirror dual gauge theory is obtained by analyzing the low energy dynamics of the S-dual brane configuration. The mirror UV gauge theory can be read by rearranging the branes along the $x^{3}$ direction, possibly using Hanany-Witten moves [9] involving the creation/annihilation of a D3-brane when an NS5-brane crosses a D5-brane, to bring the S-dual brane configuration to a configuration where the low energy gauge theory can be read using the rules summarized above. This transformation preserves the type of quiver, and thus the mirror of a linear quiver is a linear quiver and the mirror of a circular quiver is a circular quiver. ${ }^{33}$ Examples of mirror-dual pairs of quivers are given in figure 7.

An $\mathcal{N}=4$ SCFT in flat space admits canonical relevant deformations preserving $3 \mathrm{~d} \mathcal{N}=4$ Poincaré supersymmetry. These deformations are associated to the flavour symmetries $G_{C} \times G_{H}$ acting on the Coulomb and Higgs branches of the SCFT. In a UV realization of the SCFT, these deformations couple to a triplet of mass and FI parameters, which transform in the $(\mathbf{3}, \mathbf{1})$ and $(\mathbf{1}, \mathbf{3})$ of $\mathrm{SU}(2)_{C} \times \mathrm{SU}(2)_{H}$. Mass and FI deformations are obtained by deforming the UV theory with supersymmetric background vector multiplets in the Cartan of $G_{H}$ and supersymmetric background twisted vector multiplets ${ }^{34}$ in the Cartan of $G_{C}$ respectively. In the brane realization, these parameters are represented by the positions of five-branes. The position of the $i$-th D5-brane along $x^{789}$ corresponds to a mass deformation $\vec{m}_{i}$ while the position of the $i$-th NS5-brane along $x^{456}$ corresponds to an FI parameter $\vec{\xi}_{i}$. Mass and FI parameters are exchanged between mirror dual theories. Indeed, in the brane realization of mirror symmetry through S-duality the roles of the NS5 and D5-branes are exchanged. The positions of the 5-branes in the $x^{3}$ direction are irrelevant in the infrared 3d SCFT. For instance, the separation between two consecutive

\footnotetext{
${ }^{33}$ The irreducibility condition of the IR SCFT is preserved under mirror symmetry, except for a circular quiver with a single node, whose mirror dual has a single fundamental hypermultiplet.

${ }^{34}$ In twisted multiplets the roles of $\mathrm{SU}(2)_{C}$ and $\mathrm{SU}(2)_{H}$ are exchanged.
} 
NS5-branes is inversely proportional to the coupling $g_{\mathrm{YM}}^{2}$ of the effective low-energy $3 \mathrm{~d}$ SYM theory living on the D3-branes stretched between the two NS5-branes. In the deep IR, where the Yang-Mills coupling diverges, the dependence on $g_{\mathrm{YM}}^{2}$ disappears.

Linear quivers that flow to irreducible, interacting SCFT's can be labeled by two partitions of $N-\rho$ and $\hat{\rho}$ - and are denoted by $T_{\hat{\rho}}^{\rho}[\mathrm{SU}(N)]$ [29]. Circular quivers flowing to interacting SCFT's are labeled also by two partitions of $N$ and a positive integer $L$, and can be denoted by $C_{\hat{\rho}}^{\rho}[\mathrm{SU}(N), L][31] .{ }^{35}$ Under mirror symmetry

$$
T_{\hat{\rho}}^{\rho}[\mathrm{SU}(N)] \Longleftrightarrow T_{\rho}^{\hat{\rho}}[\mathrm{SU}(N)]
$$

and

$$
C_{\hat{\rho}}^{\rho}[\mathrm{SU}(N), L] \Longleftrightarrow C_{\rho}^{\hat{\rho}}[\mathrm{SU}(N), L],
$$

and the role of the two partitions are exchanged. The Coulomb branch of these theories, and by mirror symmetry the Higgs branch, describe the moduli space of monopoles in the presence of Dirac monopole singularities for linear quivers and the moduli space of instantons on a vector bundle over an ALE space for circular quivers.

In this paper we give a brane realization of both classes of loop operators discussed in section 2 and put forward an algorithm that produces a map between loop operators of mirror dual theories.

\subsection{Brane realization of Wilson loop operators}

A key ingredient in our derivation of the mirror map of loop operators is identifying a brane realization of Wilson loop operators, which are labeled by a representation $\mathcal{R}$ of the gauge group. Inserting a supersymmetric Wilson loop operator in a $3 \mathrm{~d} \mathcal{N}=4$ linear or circular quiver gauge theory admits a simple brane interpretation, obtained by enriching the setup in [9]. The construction we propose extends to $3 \mathrm{~d} \mathcal{N}=4$ gauge theories the realization of Wilson loops by branes in $4 \mathrm{~d} \mathcal{N}=4 \mathrm{SYM}$ in $[10,11]$ (see also $[33,34]$ ).

We start with the brane realization of a supersymmetric Wilson loop in the $k$-th antisymmetric representation of a $\mathrm{U}(N)$ gauge group factor in the quiver, which we denote by $\mathcal{A}_{k}$. Such an operator insertion is realized by adding $k \mathrm{~F} 1$ strings stretched in the $x^{9}$ direction ending at one end on the $N$ D3-branes where the $\mathrm{U}(N)$ gauge group is supported and at the other end on a D5' brane, defined as a D5-brane stretched in the $x^{045678}$ directions. ${ }^{36}$ The brane configuration realizing such a Wilson loop is given in table 2 . The array of fundamental strings ends between the two NS5-branes over which the N D3-branes are suspended. This brane setup is depicted in the example of figure 8-a.

This enriched brane configuration is supersymmetric: it preserves the $\mathrm{SQM}_{W}$ subalgebra of the $3 \mathrm{~d} \mathcal{N}=4$ super-Poincaré algebra discussed in section 2. Quantization of the

\footnotetext{
${ }^{35}$ In this paper we will not need the explicit mapping between the data of the quivers and $\rho, \hat{\rho}$ and $L$, but present it here for completeness. It is based on the linking numbers of the D5-branes $l_{i}$ and NS5-branes $\hat{l}_{j}$, which obey $\sum_{i}^{k} l_{i}=\sum_{j}^{\hat{k}} \hat{l}_{j}=N$, with $k$ and $\hat{k}$ the total numbers of D5/NS5-branes. For linear quivers we have that $N=l_{1}+\ldots+l_{k}=\underbrace{1+\ldots+1}_{M_{1}}+\underbrace{2+\ldots+2}_{M_{2}}+\ldots$ and $\hat{l}_{j}=N_{j-1}-N_{j}+\sum_{s=j}^{\hat{k}} M_{s}$, where $N_{0}=N_{\hat{k}}=0$ and $M_{\hat{k}}=0$. For circular quivers it is the same but with $N_{\hat{k}}=L$ and non-zero $M_{\hat{k}}$.

${ }^{36}$ Adding the D5'-brane does not break any further symmetries beyond those broken by the F1-strings.
} 


\begin{tabular}{|c|c|c|c|c|c|c|c|c|c|c|}
\hline & 0 & 1 & 2 & 3 & 4 & 5 & 6 & 7 & 8 & 9 \\
\hline D3 & $\mathrm{X}$ & $\mathrm{X}$ & $\mathrm{X}$ & $\mathrm{X}$ & & & & & & \\
D5 & $\mathrm{X}$ & $\mathrm{X}$ & $\mathrm{X}$ & & $\mathrm{X}$ & $\mathrm{X}$ & $\mathrm{X}$ & & & \\
NS5 & $\mathrm{X}$ & $\mathrm{X}$ & $\mathrm{X}$ & & & & & $\mathrm{X}$ & $\mathrm{X}$ & $\mathrm{X}$ \\
F1 & $\mathrm{X}$ & & & & & & & & & $\mathrm{X}$ \\
D5 & $\mathrm{X}$ & & & & $\mathrm{X}$ & $\mathrm{X}$ & $\mathrm{X}$ & $\mathrm{X}$ & $\mathrm{X}$ & \\
\hline
\end{tabular}

Table 2. Brane Realization of Wilson Loops in $3 \mathrm{~d} \mathcal{N}=4$ Gauge Theories.
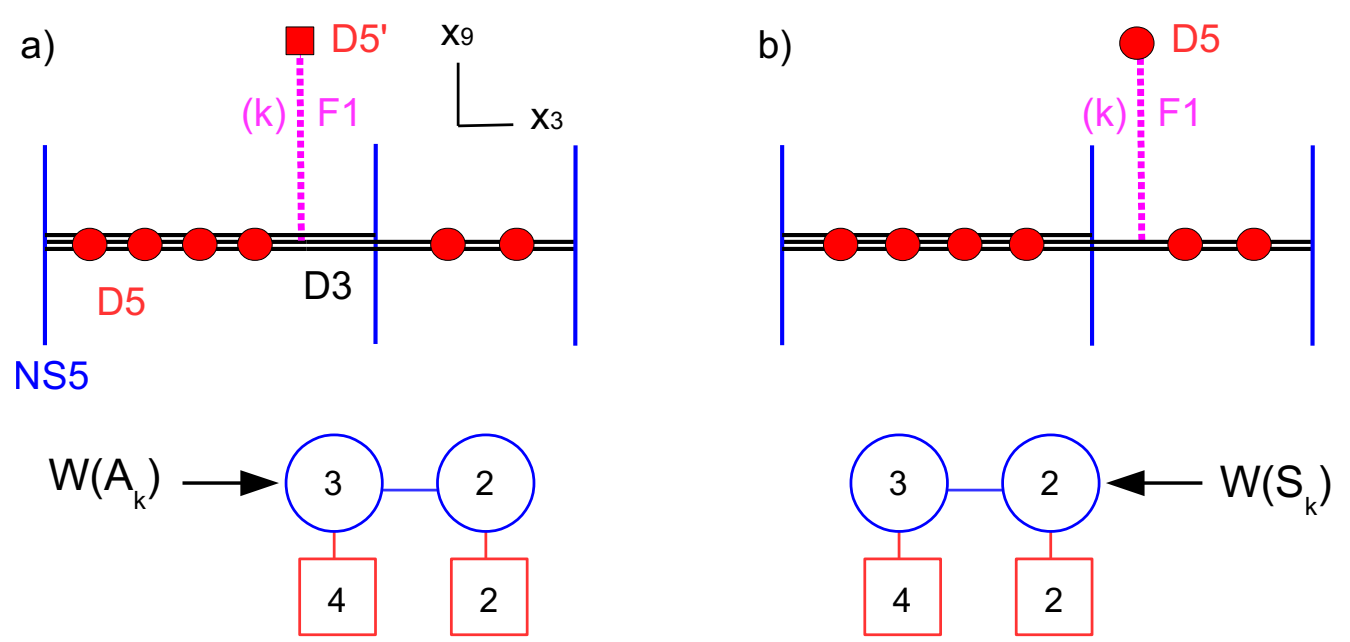

Figure 8. a) Brane configuration with $k$ F1-strings ending on a D5'-brane, realizing the insertion of a Wilson loop in the $\mathcal{A}_{k}$ representation of the $\mathrm{U}(3)$ node of a linear quiver. Here $1 \leq k \leq 3$. b) Brane configuration with $k$ F1-strings ending on a D5-brane, realizing the insertion of a Wilson loop in the $\mathcal{S}_{k}$ representation of the $\mathrm{U}(2)$ node of the same linear quiver.

open strings stretched between the D3 and the D5'-branes gives rise to 1d complex fermions $\chi$ localized on the line defect and furnish the dimensional reduction of the $2 \mathrm{~d} \mathcal{N}=(0,4)$ gauged Fermi multiplet

$$
\int d t \chi^{\dagger}\left[i \partial_{t}+\left(A_{0}+\sigma-m\right)\right] \chi
$$

where $x^{9}=m$ is the location of the D5'-brane. The fermions are in the bifundamental representation of $\mathrm{U}(N) \times \mathrm{U}(1)$, where $\mathrm{U}(N)$ is the gauge group on the D3-brane segment where the F1-strings end and the U(1) is the flavour symmetry associated to the D5'-brane. The fermions can be integrated out exactly and yield ${ }^{37}$

$$
Z_{0}=\frac{1}{\sqrt{\operatorname{det} \mathrm{U}}} \sum_{l=0}^{N} e^{-i \beta m l} \operatorname{Tr}_{\mathcal{A}_{l}} U
$$

where $U=P e^{i \int_{0}^{\beta} d t A_{0}+\sigma}$ is the supersymmetric holonomy operator. As it stands there is a global anomaly for the $\mathrm{U}(1) \subset \mathrm{U}(N)$, since under large gauge transformations $Z_{0} \rightarrow-Z_{0}$.

\footnotetext{
${ }^{37}$ Here we put the system on a circle of length $\beta$.
} 
Our brane realization of the Wilson loop, however, engineers a bare supersymmetric ChernSimons term at level $k=1 / 2$, that is $1 / 2 \int\left(A_{0}+\sigma\right)$, which precisely cancels the offending factor $\operatorname{det}(\mathrm{U})^{-1 / 2}$, and the brane system is anomaly free. ${ }^{38}$ Integrating out the fermions in the presence of the Chern-Simons term therefore yields

$$
Z=\sum_{l=0}^{N} e^{-i \beta m l} \operatorname{Tr}_{\mathcal{A}_{l}} U .
$$

The presence of $k$ F1-strings stretched between the D3 and D5'-branes is represented in the gauge theory by the insertion of $k$ creation operators for these fermions in the past and $k$ annihilation operators in the future. Physically, these operators insert a charged probe into the gauge theory. Integrating out these fermions inserts a supersymmetric Wilson loop operator in the $k$-th antisymmetric representation [10]

$$
\frac{\left\langle\left(\chi^{\dagger}(0)\right)^{k} \chi^{k}(\beta)\right\rangle}{Z}=e^{-i \beta m k} \operatorname{Tr}_{\mathcal{A}_{k}} U .
$$

The weights of the $k$-th antisymmetric representation of $\mathrm{U}(N)$ admit an elegant description in the brane construction. We must distribute $k$ F1-strings among $N$ D3-branes (all $k \mathrm{~F} 1$-strings terminate at the other end on a single D5'-brane). To a pattern of $k$ F1-strings where $k_{j}$ strings end on the $j$-th D3-brane (see figure 9) we associate a set of $N$ non-negative integers $\left\{k_{j}\right\}$ obeying $k=k_{1}+k_{2}+\cdots+k_{N}$, with $k_{j} \geq 0$ for all $j$. However, not all positive integers $k_{j}$ are allowed. There can be at most one F1-string stretched between a D3-brane and a D5'-brane. This is the so-called s-rule [9], and is a constraint that follows from Pauli's exclusion principle [36]. Therefore, the allowed configurations are described by a collection of $N$ non-negative integers $\left\{k_{j}\right\}$ with the constraint that $k_{j} \leq 1$. This set of configurations is in one-to-one correspondence with the weights of the $k$-th antisymmetric representation of $\mathrm{U}(N)$, i.e. of $\mathcal{A}_{k}$.

We now turn to a Wilson loop in the $k$-th symmetric representation of $\mathrm{U}(N)$, which we denote by $\mathcal{S}_{k}$. Inserting a Wilson loop in the $k$-th symmetric representation is realized by adding $k \mathrm{~F} 1$ strings stretched in the $x^{9}$ direction ending at one end on the $N$ D3-branes where the $\mathrm{U}(N)$ gauge group is supported and at the other end on a D5-brane stretched in the $x^{012456}$ directions and localized in the $x^{9}$ direction. The array of fundamental strings ends between the two NS5-branes over which the N D3-branes are suspended. This setup is illustrated in figure 8-b. In this case the charged probe particle inserted by the Wilson loop can be though of as arising from a very heavy hypermultiplet, represented by adding a D5-brane to the theory and then taking the D5-brane far away from the stack, thus giving it a large mass and making the hypermultiplet fields nonrelativistic. Integrating out the heavy hypermultiplet in the presence of $k$ heavy insertions yields a supersymmetric Wilson loop operator in the $k$-th symmetric representation $[10,11] .{ }^{39}$

\footnotetext{
${ }^{38}$ This coupling is obtained by inserting the flux produced by the D5'-brane on the non-abelian ChernSimons term on the worldvolume of the D3-branes. This is T-dual to the haf-integral Chern-Simons term discussed in [35].

${ }^{39}$ In [11] the heavy charged particle was obtained by going to the Coulomb branch while here by giving a large mass to a hypermultiplet.
} 


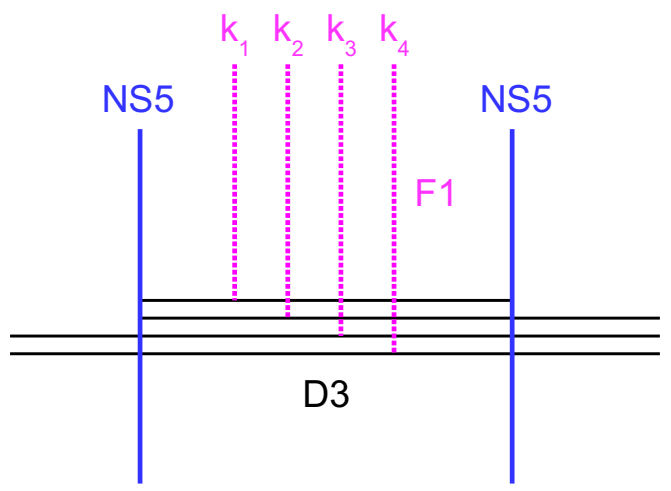

Figure 9. Configuration with stacks of $k_{j}$ F1-strings, $1 \leq j \leq 4$, ending on each of the four D3-branes, associated to a weight $\left(k_{1}, k_{2}, k_{3}, k_{4}\right)$ of a representation of $\mathrm{U}(4)$.

The weights of the $k$-th symmetric representation of $\mathrm{U}(N)$ admit an elegant description in the brane construction. We again distribute $k$ F1-strings among $N$ D3-branes (all $k \mathrm{~F} 1-$ strings terminate at the other end on a single D5-brane). To a pattern of $k$ F1-strings where $k_{j}$ strings end on the $j$-th D3-brane (see figure 9) we associate a set of non-negative integers $\left\{k_{j}\right\}$ obeying $k=k_{1}+k_{2}+\cdots+k_{N}$, with $k_{j} \geq 0$ for all $j$. In this case an arbitrary number of F1-strings can be stretched between the D5 and a D3-brane. Therefore the set of configurations is in one-to-one correspondence with the weights of the $k$-th symmetric representation of $\mathrm{U}(N)$, i.e. of $\mathcal{S}_{k}$.

Our brane construction can be easily generalized to Wilson loops in the tensor product of an arbitrary number of symmetric and antisymmetric representations of $\mathrm{U}(N): \mathcal{R}=$ $\otimes_{a=1}^{d} \mathcal{S}_{k^{(a)}} \otimes_{b=1}^{d^{\prime}} \mathcal{A}_{l^{(b)}}$. This requires considering F1-strings stretched between $d$ D5-branes and $d^{\prime}$ D5'-branes and the $N$ D3-branes that support the gauge group. For the above mentioned representation $k^{(a)}$ F1-strings must emanate from the $a$-th D5-brane and $l^{(b)}$ F1-strings from the $b$-th D5'-brane. Integrating out the massive charged particles produced by this configuration yields a Wilson loop in the desired representation. Furthermore, the set of allowed F1-string configurations, labeled by $\left\{k_{j}^{(1)}, \ldots, k_{j}^{(d)}, l_{j}^{(1)}, \ldots, l_{j}^{\left(d^{\prime}\right)}\right\}_{j=1 \ldots N}$ with $k_{j}^{(a)} \in \mathbb{N}, l_{j}^{(b)} \in\{0,1\}$ and such that $\sum_{j} k_{j}^{(a)}=k^{(a)}$ and $\sum_{j} l_{j}^{(b)}=l^{(b)}$ yields precisely all the weights in the representation $\mathcal{R}=\otimes_{a=1}^{d} \mathcal{S}_{k^{(a)}} \otimes_{b=1}^{d^{\prime}} \mathcal{A}_{l^{(b)}}$ of $\mathrm{U}(N)$. More precisely a configuration is associated to a weight $w=\left(w_{1}, w_{2}, \cdots, w_{N}\right)$ in the orthogonal basis with $w_{j}=\sum_{a=1}^{d} k_{j}^{(a)}+\sum_{b=1}^{d^{\prime}} l_{j}^{(b)}$. Our analysis can be summarized in table 3.

In order to describe Wilson loops in the above mentioned representation we have to place the $d$ D5 and $d^{\prime}$ D5'-branes at different positions in the $x^{3}$ and $x^{9}$ directions. The separation in the $x^{9}$ direction is not essential at this stage but plays a role when we identify the S-dual brane configuration and the mirror dual Vortex loop. The separation in the $x^{3}$ direction is more crucial: if two D5-branes sit at the same $x^{3}$ position, the pattern of $\mathrm{F} 1$ strings is more complicated since strings can now break and be stretched between the two D5-branes preserving the same amount of supersymmetry. In this case we expect that the brane configuration would insert a Wilson loop in an irreducible representation of $\mathrm{U}(N)$, as in $[10,11]$. An irreducible representation $\mathcal{R}$ of $\mathrm{U}(N)$ labeled by a Young diagram with 


\begin{tabular}{|c|c|c|}
\hline number of D5 & number of D5' & rep. $\mathcal{R}$ \\
\hline 1 & 0 & $\mathcal{S}_{k}$ \\
0 & 1 & $\mathcal{A}_{k}$ \\
0 & 2 & $\mathcal{A}_{l^{(1)}} \otimes \mathcal{A}_{l^{(2)}}$ \\
1 & 1 & $\mathcal{S}_{k} \otimes \mathcal{A}_{l}$ \\
\hline
\end{tabular}

Table 3. D5/D5'-brane arrays inserting a Wilson loop in a representation $\mathcal{R}$ of $\mathrm{U}(N)$. The integers $k^{(a)}$ and $l^{(b)}$ correspond to numbers of F1-strings stretched between a single 5-brane and the $N$ D3-branes as explained in the text.
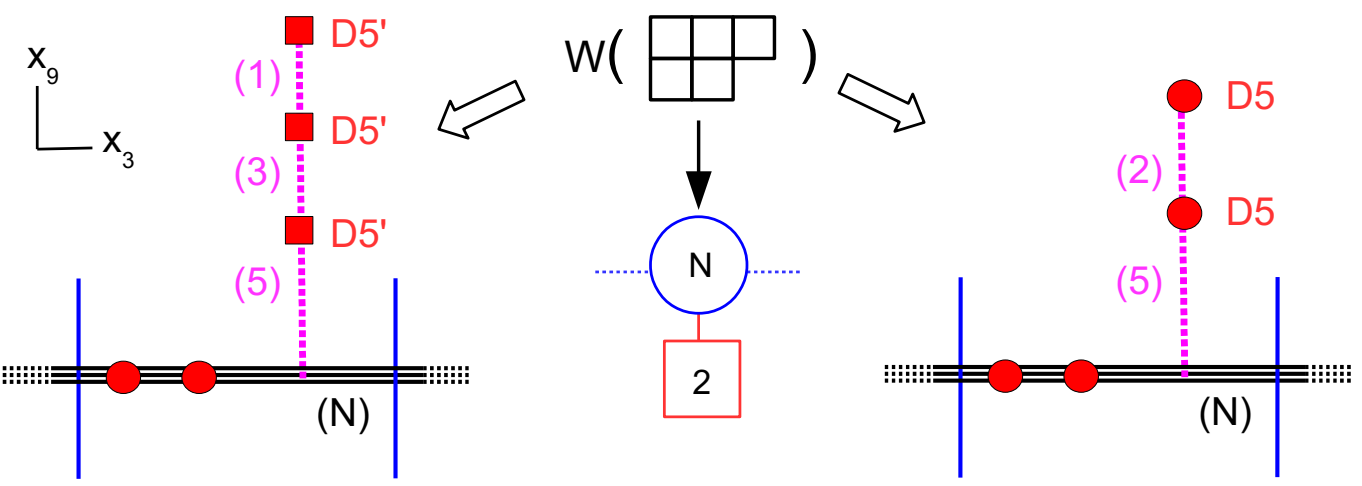

Figure 10. The Wilson loop in the representation of $\mathrm{U}(N)$ labeled by the Young tableau of the figure can be realized in two ways. On the left: 1, 2 and 2 strings emanate respectively from three coincident D5'-branes (along $x^{3}$ ). On the right: 2 and 3 strings emanate respectively from two coincident D5-branes.

$M$ rows and $L$ columns

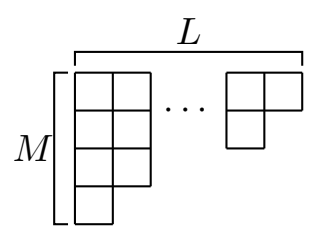

is realized either by having $|\mathcal{R}|$ F1-strings end on $M$ coincident D5-branes (in the $x^{3}$ direction) or $|\mathcal{R}|$ F1-strings end on $L$ coincident D5'-branes away from the main stack, where $|\mathcal{R}|$ is the number of boxes in the Young diagram corresponding to $\mathcal{R}$. Figure 10 provides an example of the two possible brane configurations realizing a Wilson loop.

In the brane realization we must specify between which two consecutive NS5-branes the F1-strings end. This determines in which gauge group the Wilson loop is inserted. While the F1-strings cannot be moved across an NS5-brane without changing the Wilson loop operator, they can be freely moved across a D5-brane without changing the Wilson loop operator in the IR if the D5-brane has the same number of D3-branes ending on the left and the right, which is the case in the canonical brane configuration in figure 6 . The corresponding S-dual statement, that D1-branes can be moved across an NS5-brane with the same number of D3-branes ending on the left and the right but that D1-branes cannot 


\begin{tabular}{|c|c|c|c|c|c|c|c|c|c|c|}
\hline & 0 & 1 & 2 & 3 & 4 & 5 & 6 & 7 & 8 & 9 \\
\hline D3 & $\mathrm{X}$ & $\mathrm{X}$ & $\mathrm{X}$ & $\mathrm{X}$ & & & & & & \\
D5 & $\mathrm{X}$ & $\mathrm{X}$ & $\mathrm{X}$ & & $\mathrm{X}$ & $\mathrm{X}$ & $\mathrm{X}$ & & & \\
NS5 & $\mathrm{X}$ & $\mathrm{X}$ & $\mathrm{X}$ & & & & & $\mathrm{X}$ & $\mathrm{X}$ & $\mathrm{X}$ \\
D1 & $\mathrm{X}$ & & & & & & $\mathrm{X}$ & & & \\
NS5, & $\mathrm{X}$ & & & & $\mathrm{X}$ & $\mathrm{X}$ & & $\mathrm{X}$ & $\mathrm{X}$ & $\mathrm{X}$ \\
\hline
\end{tabular}

Table 4. Brane array with D1-branes.

be moved across a D5-brane without changing the IR dynamics, will play an important role in unraveling the mirror map of loop operators.

\subsection{Mirror of Wilson loops from S-duality}

After having found a brane realization of Wilson loops, we now make use of the fact that mirror symmetry corresponds to S-duality in the Type IIB brane realization to derive the mirror dual of supersymmetric Wilson loop operators. Here we loosely call S-duality what is really $\mathrm{S}$-duality combined with the rotation that sends $x^{456}$ to $x^{789}$ and $x^{789}$ to $-x^{456}$, so that D5-branes and NS5-branes get exchanged. We shall see that the information about the representation of the Wilson loop is encoded in the discrete data of a $1 \mathrm{~d} \mathcal{N}=4 \mathrm{SQM}$ quiver quantum mechanics gauge theory.

We have found that Wilson loop insertions can be realized by a brane configuration with F1-strings stretched between the N D3-branes and D5-branes and/or D5'-branes, oriented as in table 2. Under S-duality the D5 and D5'-branes become NS5 and NS5'-branes, while the F1-strings become D1-branes, oriented as in table 4. This brane configuration preserves the $\mathrm{SQM}_{V}$ subalgebra of the $3 \mathrm{~d} \mathcal{N}=4$ super-Poincaré algebra discussed in section $2 .{ }^{40}$ A $1 \mathrm{~d} \mathcal{N}=4 \mathrm{SQM}_{V}$ invariant theory is supported on the D1-branes. ${ }^{41}$ The $\mathrm{U}(1) \times \mathrm{SU}(2)$ R-symmetry of $\mathrm{SQM}_{V}$ is identified with $\mathrm{SO}(2)_{12} \times \mathrm{SO}(3)_{789}$ rotations in spacetime. ${ }^{42}$ The remaining $\mathrm{SO}(2)_{12}-\mathrm{SO}(2)_{45}$ isometry (a diagonal $\mathrm{SO}(2)$ ) is mapped to a flavor symmetry of the SQM theory, which is the commutant of $\mathrm{SQM}_{V}$ in the $3 \mathrm{~d} \mathcal{N}=4$ supersymmetry algebra.

We will now exhibit that Wilson loops in a UV $3 \mathrm{~d} \mathcal{N}=4$ gauge theory are mirror to loop operators defined by $1 \mathrm{~d} \mathcal{N}=4 \mathrm{SQM}_{V}$ invariant quiver gauge theories coupled to the mirror $3 \mathrm{~d} \mathcal{N}=4$ gauge theory. Likewise, the Wilson loops of the mirror theory are mapped to $1 \mathrm{~d} \mathcal{N}=4$ quiver gauge theories coupled to the original $3 \mathrm{~d} \mathcal{N}=4$ gauge theory. The $1 \mathrm{~d} \mathcal{N}=4$ quiver gauge theories and the way they couple to the $3 \mathrm{~d} \mathcal{N}=4$ gauge theory are found by identifying the IR gauge theories living on the D1-branes in the S-dual brane configuration.

How can the mirror operator to a given Wilson loop be constructed? First we perform S-duality on the brane configuration realizing a Wilson loop in a $3 \mathrm{~d} \mathcal{N}=4$ gauge theory.

\footnotetext{
${ }^{40}$ S-duality in Type IIB string theory acts nontrivially on the supersymmetry charges.

${ }^{41} \mathrm{D} 1$-branes span an interval in the $x^{6}$ direction. The low-energy effective theory is thus one-dimensional.

${ }^{42}$ For later convenience, we have combined the R-symmetry $R_{H}$ realized by $\mathrm{SO}(2)_{45}$ with the with the $\mathrm{SO}(2)_{12}-\mathrm{SO}(2)_{45}$ commutant to define a new R-symmetry: $\mathrm{SO}(2)_{12}$.
} 


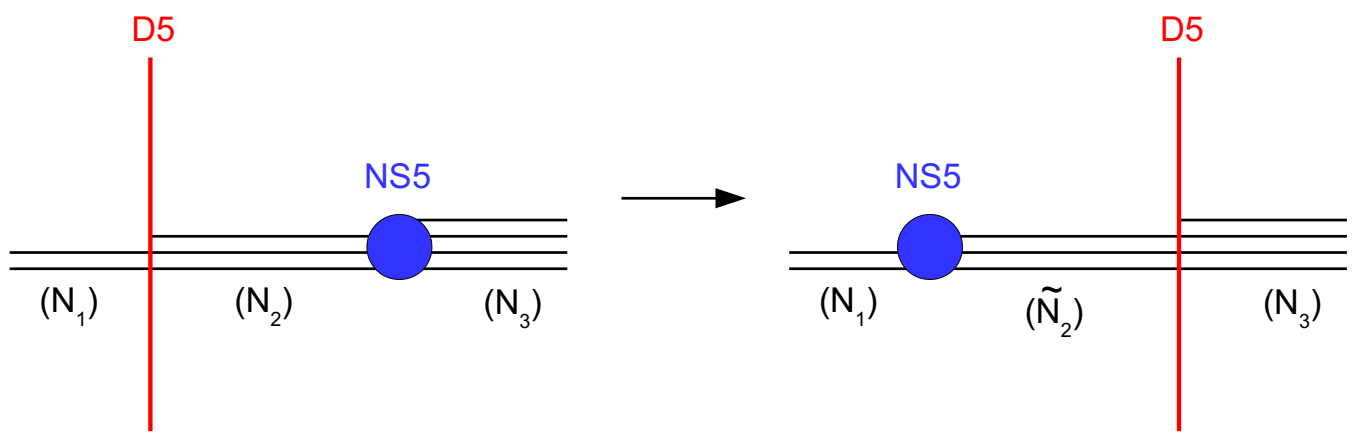

Figure 11. Hanany-Witten five-brane move. Initially $N_{1}<N_{2}$. After the D5 has crossed the NS5, the number of D3-branes between them is $\widetilde{N}_{2}=N_{1}+N_{3}-N_{2}+1$. The D3-excess on the D5-brane sides goes down from $N_{2}-N_{1}$ to $N_{2}-N_{1}-1$.

In the absence of the F1-strings, the mirror $3 \mathrm{~d} \mathcal{N}=4$ theory is found by rearranging the S-dual brane configuration so as to bring it to the canonical frame explained at the beginning of section 3, where the mirror gauge theory can be easily read. After S-duality, the number of D3-branes on the left and on the right of a D5-brane need not be the same while that number is the same for every NS5-brane, as before S-duality the D5-branes had the same number of D3-branes on both sides. In order to bring the brane configuration to one where the gauge theory can be read we must move the D5-branes with an excess of D3-branes in the direction of the excess and make it pass through NS5-branes. Every such move, exchanging a D5-brane with an NS5-brane, results in the creation of a D3-brane [9] on the side of the D5-brane that had a smaller number of D3-branes, thus diminishing the D3-brane excess. See figure 11. This process must be continued until the number of D3-branes on each of the D5-branes is the same on the left and on the right. From this final configuration we can read the mirror dual gauge theory.

Insertion of a Wilson loop operator in a gauge group factor requires adding F1-strings ending between the two consecutive NS5-branes where the D3-branes supporting that gauge factor are suspended. After S-duality, we have the same S-dual brane configuration as before but now with extra D1-branes ending between a pair of consecutive D5-branes. It is from this S-dual brane configuration that we read off the $1 \mathrm{~d} \mathcal{N}=4$ quiver gauge theory living on the D1-branes and its coupling to the mirror dual gauge theory. In order to read the mirror description of the original Wilson loop we once again move the D5-branes in the direction of D3-brane excess to bring the brane configuration to the canonical one, where the $3 \mathrm{~d} \mathcal{N}=4$ mirror gauge theory can be extracted. During this process we do not allow a D5-brane to cross a D1-brane. In the simplest situations, the D5-branes can be moved to the canonical configuration of the mirror dual theory without having to move the D1-branes. Then we can directly read off the $1 \mathrm{~d} \mathcal{N}=4$ quiver theory from the final brane configuration with D1-branes (see below). In more complicated examples, we have a situation when a D5-brane must be moved past an NS5-brane while D1-branes stand between them. For definiteness let us imagine that the D5-brane must be moved from the left to the right of an NS5-brane, with the D1-branes in between, as in figure 12-a. 
a)

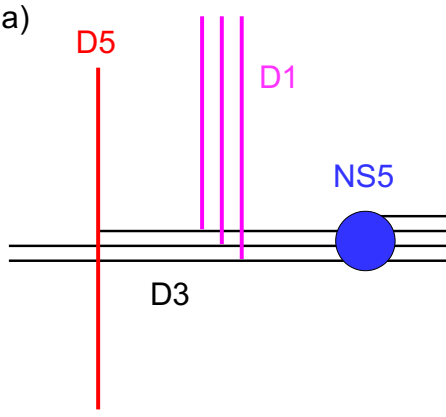

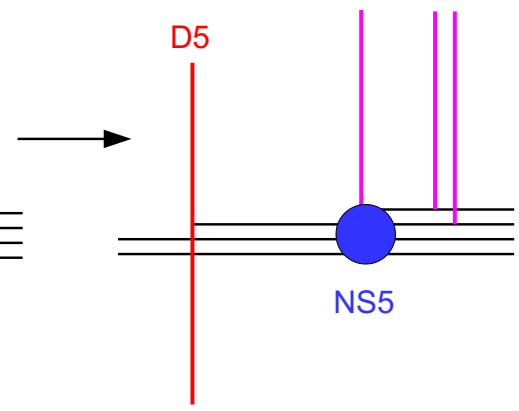

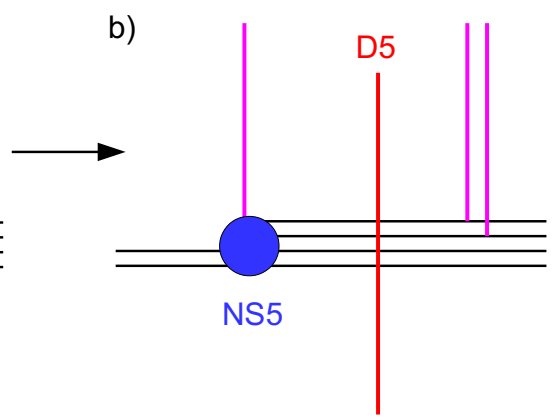

Figure 12. a) The D5-brane has to be moved to the right of the NS5-brane to reach the canonical brane configuration. The D1-branes in between must first be moved across the NS5-brane themselves. b) After moving the D1 and D5-branes, stacks of D1-branes end up attached to the NS5-brane and moved away from the main brane configuration along transverse directions (transverse to the picture).

In this situation the D1-branes together with the NS5/NS5'-branes to which they are attached at the other end must be moved first to the right of the NS5-brane. Here we must distinguish two situations: if the number of D3-branes ending on both sides of the NS5-brane are equal, then the D1-branes can be moved across it without anything special happening (in the S-dual picture, F1-strings can cross a D5-brane with the same number of D3-branes on both sides in the same trivial way). However the NS5-brane can also have an excess of D3-branes on the right. ${ }^{43}$ Then the NS5-brane develops a D3-brane spike [37] and some D1-branes may be moved along the spike so that they end on the NS5-brane, and can then be moved far away from the main stack along transverse directions. If there were $k$ D1-branes in the original stack, they could split into $k_{1}$ D1-branes moved from the left to the right of the NS5-brane along the D3-branes and $k_{2}$ D1-branes moved along the NS5-brane spike and away from the main stack, with $k=k_{1}+k_{2}$. Then the D5-brane can be moved across the NS5-brane as usual, without encountering the D1-branes. This is illustrated in figure 12-b.

The choice of splitting $k=k_{1}+k_{2}$ may be constrained, but generically several splittings may be allowed. The idea of the mirror map is that the initial Wilson loop is mapped to the sum of the Vortex loops realized by the possible final brane configurations, weighted with coefficients. The precise rules to derive which final brane configurations can be reached (and their weighting coefficients) are not obvious. Intuitively the difficulty is related to the fact that the D3-spike is "on the wrong side" of the NS5-branes, so that we cannot directly move the D1-branes along the spikes. We will see shortly that the situation is under

\footnotetext{
${ }^{43}$ The NS5-brane cannot have an excess of D3-branes on the left, where the D5-brane is coming from. This can be understood as follows. After S-duality of the original configuration, the number of D3-branes on both sides of the NS5-brane are equal. During the brane rearrangement some D5-branes can move across it but only in one direction (because D5-branes do not cross between themselves during the rearrangement). In our example, D5-branes cross the NS5-brane from the left to the right. For each D5-brane crossing the NS5, the Hanany-Witten rule implies that the excess of D3-branes on the right of the NS5 increases by one, so that along the process the NS5 develops an excess of D3-branes on the right. In the other case when D5-branes cross the NS5 from the right to the left, the NS5 develops an excess of D3-branes on the left.
} 
much better control starting from the D1-branes configurations realizing Vortex loops and $S$-dualizing to configurations realizing the mirror Wilson loops.

What are the final brane configurations? In addition to the D3, D5, NS5 system realizing the mirror dual theory, there are D1-branes ending on D3-branes on one side and on NS5 and/or NS5'-branes situed far away from the main configuration. Moreover there are also D1-branes ending on NS5-branes in the main stack. The physical interpretation of having $q$ D1-branes ending on an NS5-brane is as a background Wilson loop of charge $q$ for a U(1) global symmetry associated to the NS5-brane, which is a combination of the so-called topological symmetries of the $3 \mathrm{~d}$ theory. These flavor Wilson loops combine with the Vortex loop realized by the D1-branes ending on D3-branes, which we describe now.

A final brane configuration with D1-branes ending on D3-branes in one end and on NS5 and/or NS5'-branes on the other can be used to give at least two descriptions of the mirror of a Wilson loop. This brane configuration can be thought of as a deformation of two other brane configurations, both of which are conducive to reading off the $1 \mathrm{~d} \mathcal{N}=4$ quiver gauge theory living on the D1-branes and its couplings to the bulk $3 \mathrm{~d} \mathcal{N}=4$ gauge theory. The D1-branes can be either moved to the nearest NS5-brane to the left or to the nearest NS5-brane to the right. In order to reach this configuration, from which the gauge theory description of the mirror loop operators can be read off, we must again ensure that no D1-strings cross D5-branes. This means that we have to move the D5-branes between the D1-branes and the nearest NS5-brane to the other side of that NS5-brane. In summary, we can realize the mirror loop operator either as:

1. Deformation of the coupled $3 \mathrm{~d} / 1 \mathrm{~d}$ theory realized by the D1-branes when they end on the NS5-brane on the left.

2. Deformation of the coupled $3 \mathrm{~d} / 1 \mathrm{~d}$ theory realized by the D1-branes when they end on the NS5-brane on the right.

These yield two dual descriptions of the same operator.

Once we move the stack of D1-branes so that it ends on a neighbouring NS5-brane, we can read off the $1 \mathrm{~d} \mathcal{N}=4$ quiver gauge theory and how it couples to the $3 \mathrm{~d} \mathcal{N}=4$ gauge theory. The $1 \mathrm{~d} \mathcal{N}=4$ gauge theory associated to a brane configuration is constructed by assigning:

- A $\mathrm{U}(k)$ vector multiplet to $k$ D1-branes suspended between an NS5-brane and an NS5'-brane.

- $\mathrm{A} \mathrm{U}(k)$ vector multiplet and an adjoint chiral multiplet ${ }^{44}$ to $k$ D1-branes suspended between two NS5-branes or two NS5'-branes.

- Two chiral multiplets in the bifundamental and anti-bifundamental representations of $\mathrm{U}\left(k_{1}\right) \times \mathrm{U}\left(k_{2}\right),{ }^{45}$ to an NS5-brane or NS5'-brane with $k_{1}$ D1-branes ending on its left and $k_{2}$ D1-branes ending on its right.

\footnotetext{
${ }^{44}$ The adjoint chiral multiplet describes the position of the D1-branes in the $x^{12}$ directions (for NS5branes), or in the $x^{45}$ directions (for NS5'-branes).

${ }^{45}$ The bifundamental representation of $\mathrm{U}\left(k_{1}\right) \times \mathrm{U}\left(k_{2}\right)$ is $\left(\mathbf{k}_{\mathbf{1}}, \overline{\mathbf{k}}_{\mathbf{2}}\right)$ and the anti-bifundamental is its complex conjugate.
} 

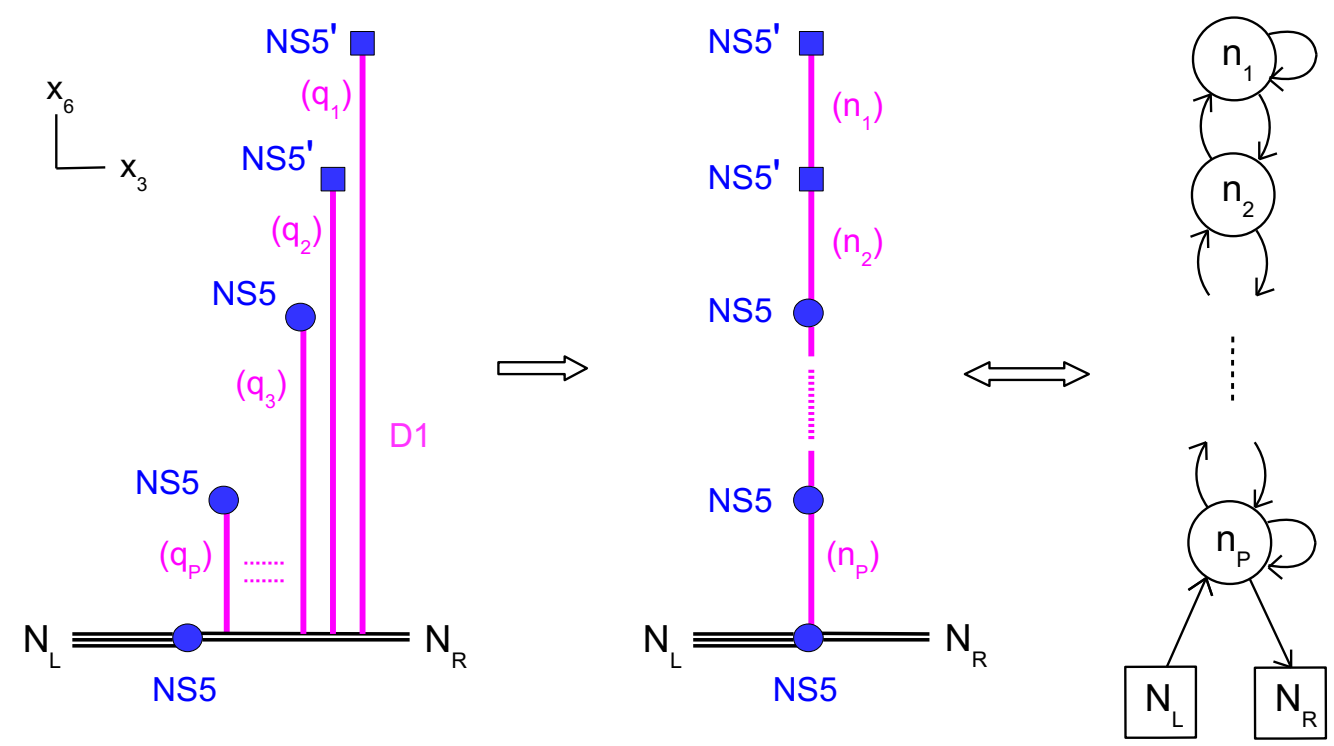

Figure 13. To read the SQM quiver we move the D1-branes to the closest NS5-brane on the left. The rank of nodes are given by $n_{j}=\sum_{i=1}^{j} q_{i}$, where $q_{i}$ is the number of D1-branes emanating from the $i$-th five-brane. The $q_{i}$ are trivially mapped to the $k^{(a)}$ and $l^{(b)}$ introduced in the text. NS5'-branes are denoted by blue squares, as a opposed to NS5-branes that are blue circles.

- A chiral multiplet in the bifundamental of $\mathrm{U}\left(N_{R}\right) \times \mathrm{U}(k)$ and an chiral multiplet in the bifundamental of $\mathrm{U}(k) \times \mathrm{U}\left(N_{L}\right)$ to $k$ D1-branes ending on an NS5-brane which has $N_{L}$ D3-branes ending on its left and $N_{R}$ D3-branes ending on its right.

The parameters of the $1 \mathrm{~d} \mathcal{N}=4$ gauge theory living on the D1-branes admit a brane interpretation. The gauge coupling of the vector multiplet realized on D1-branes stretched between two five-branes (NS5 or NS5') is inversely proportional to the distance in the $x^{6}$ direction between the five-branes. The relative position in the $x^{3}$ direction between two consecutive five-branes (NS5 or NS5') determines the $1 \mathrm{~d} \mathcal{N}=4$ FI parameter for the corresponding gauge group factor. If we denote the position of the NS5-brane in the main stack where the D1-strings originally end by $x_{0}^{3}$ and $x_{i}^{3}$ the position of the $i$-th five-brane away from the main stack, the $1 \mathrm{~d}$ FI parameters are given by $\eta_{i}=x_{i}^{3}-x_{i-1}^{3}$. In particular, if the D1-branes are moved to the right of the NS5-brane in the main stack along the D3-branes then the FI parameter for the first 1d gauge group factor is positive, while it is negative if we move the D1-branes to the left along the D3-branes. Therefore, the $3 \mathrm{~d} / 1 \mathrm{~d}$ gauge theories 1) and 2) described above obtained after S-duality should be thought of as a deformation with $\eta_{1}>0$ of theory 1) and a deformation with $\eta_{1}<0$ of theory 2).

Consider for instance the D1-brane configuration of figure 13 near an NS5-brane in the main stack with $k^{(a)}$ D1-strings emanating from the $a$-th NS5-brane and $l^{(b)}$ D1-strings from the $b$-th NS5'-brane and ending on the $N_{R}$ D3-branes to the right of the NS5-brane in the main stack. There are also $N_{L}$ D3-branes to the left of this NS5-brane. To this brane configuration we can associate the representation $\mathcal{R}=\otimes_{a=1}^{d} \mathcal{S}_{k^{(a)}} \otimes_{b=1}^{d^{\prime}} \mathcal{A}_{l^{(b)}}$ of $\mathrm{U}\left(N_{R}\right)$, where $d$ and $d^{\prime}$ denote the numbers of NS5 and NS5'-branes from which the D1-branes emanate. This brane configuration can be thought of as a deformation of a $1 \mathrm{~d} \mathcal{N}=4$ 
quiver gauge theory in figure 13 with positive FI parameters. There are actually different dual descriptions of the $1 \mathrm{~d} \mathcal{N}=4$ theories depending on the relative order of the $d$ NS5 and $d^{\prime}$ NS5'-branes in the $x^{6}$ direction. Different relative positions give rise to different dual $1 \mathrm{~d} \mathcal{N}=4$ descriptions of the same Vortex loop operator labeled by the representation $\mathcal{R}=\otimes_{a=1}^{d} \mathcal{S}_{k^{(a)}} \otimes_{b=1}^{d^{\prime}} \mathcal{A}_{l^{(b)}}$ of $\mathrm{U}\left(N_{R}\right)$. Roughly speaking, these dual descriptions are related by a $1 \mathrm{~d} \mathcal{N}=4$ version of Seiberg duality [20] (see also [21, 38]).

We have described the $1 \mathrm{~d} \mathcal{N}=4 \mathrm{SQM}_{V}$ quiver theory living on the D1-branes. We must now explain how it is coupled to the $3 \mathrm{~d} \mathcal{N}=4$ gauge theory. The idea is that the $\mathrm{U}\left(N_{L}\right) \times \mathrm{U}\left(N_{R}\right)$ flavor symmetry of the $1 \mathrm{~d} \mathcal{N}=4$ theory is gauged with $3 \mathrm{~d}$ bulk fields living on the D3-branes ending on the NS5-brane on the main stack. We must, however, distinguish the D3-branes supporting dynamical gauge fields from the non-dynamical D3branes stretched between the NS5 and a D5-brane. To read the SQM theory we had to move D1-branes to the closest NS5-brane to left (or to the right). Suppose there are $n_{\mathrm{D} 5}$ D5-branes standing between this NS5 and the D1-branes, then we have to move them first to the left of the NS5 and, by the Hanany-Witten effect, one D3-brane per D5 is created ending on the left of the NS5-brane. In this case the $N_{L}$ D3-branes ending on the left of the NS5 decompose into $N_{L}=n_{\mathrm{D} 5}+n_{\mathrm{D} 3}$, where $n_{\mathrm{D} 3}$ is the number of dynamical D3branes, those supporting a $\mathrm{U}\left(n_{\mathrm{D} 3}\right) 3 \mathrm{~d} \mathcal{N}=4$ vector multiplet. On the other side, the $N_{R}$ D3-branes ending on the right of the NS5 support a $\mathrm{U}\left(N_{R}\right) 3 \mathrm{~d} \mathcal{N}=4$ vector multiplet. The $1 \mathrm{~d} \mathcal{N}=4$ theory is then coupled to the $3 \mathrm{~d} \mathcal{N}=4$ theory by gauging the $\mathrm{U}\left(N_{R}\right)$ and $\mathrm{U}\left(n_{\mathrm{D} 3}\right) \subset \mathrm{U}\left(N_{L}\right)$ flavor symmetries on the defect with dynamical $3 \mathrm{~d} \mathcal{N}=4$ vector multiplets. ${ }^{46}$ Furthermore, there is a cubic superpotential as in (2.12) which breaks the $\mathrm{U}\left(n_{\mathrm{D} 5}\right)_{3 \mathrm{~d}} \times \mathrm{U}\left(n_{\mathrm{D} 5}\right)_{1 \mathrm{~d}}$ flavor symmetry to the diagonal $\mathrm{U}\left(n_{\mathrm{D} 5}\right)$, where $\mathrm{U}\left(n_{\mathrm{D} 5}\right)_{1 \mathrm{~d}} \subset \mathrm{U}\left(N_{L}\right)$ and $\mathrm{U}\left(n_{\mathrm{D} 5}\right)_{3 \mathrm{~d}}$ is the $3 \mathrm{~d}$ flavor symmetry acting on the $n_{\mathrm{D} 5}$ hypermultiplets associated to the D5-branes. The $3 \mathrm{~d} / 1 \mathrm{~d}$ coupling that can read from the brane picture is summarized in the example of figure 14 .

The 3d/1d coupling for the $\mathrm{SQM}_{V}$ theories read from moving the D1s to the nearest NS5 on the right are found similarly, as shown in figure 15.

As mentioned above, it turns out to be simpler to derive the mirror map if we consider the inverse problem of finding the combination of Wilson loops dual to a given Vortex loop. Starting with a configuration of D1-branes realizing a Vortex loop, we can S-dualize to obtain a configuration with F1-strings and move the D5-branes to reach the canonical brane configuration of the mirror-dual theory. The crucial difference is that we do not need to move the F1-strings, instead we are allowed to move the D5-branes across the stack of F1-strings if this is necessary to reach the canonical configuration. When we have to move a D5 across the F1-strings the situation is always the same, namely the D5-brane has an excess of D3-branes on the side toward which it is moving. This means that the F1-strings are on the side of the D3-spikes, along which they can be moved without obstruction. When the D5-brane crosses the F1-strings the number of D3-branes at the bottom of the

\footnotetext{
${ }^{46}$ More precisely the $3 \mathrm{~d} \mathcal{N}=4$ vector multiplet decomposes into multiplets of the subalgebra preserved by $\mathrm{SQM}_{V}$, each multiplet containing fields at a given position in the plane orthogonal to the defect. A $1 \mathrm{~d}$ $\mathcal{N}=4 \mathrm{SQM}_{V}$ vector multiplet embedded in the $3 \mathrm{~d} \mathcal{N}=4$ vector multiplet lives at the position of the defect and gauges the corresponding flavor symmetry.
} 

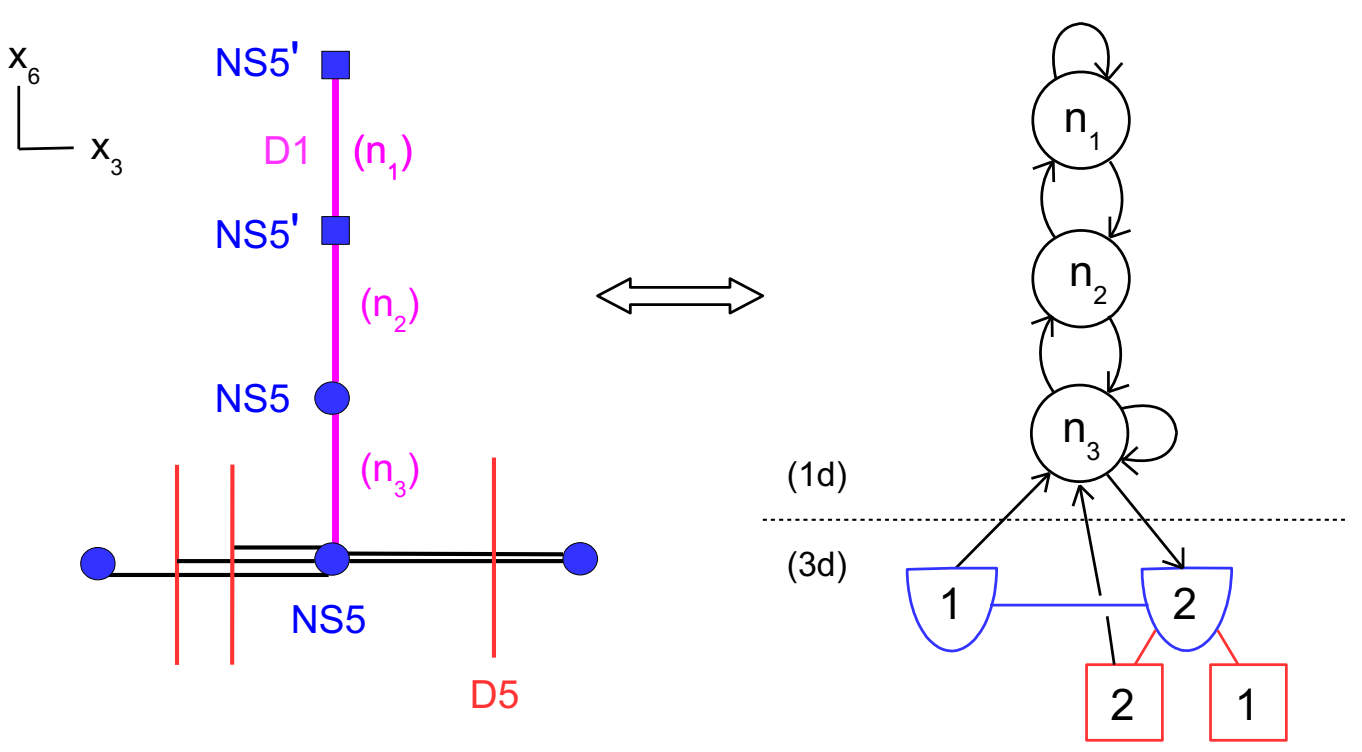

Figure 14. Example of configuration with D1-branes and associated $1 \mathrm{~d} \mathcal{N}=4$ quiver gauge theory, coupled to the $3 \mathrm{~d} \mathcal{N}=4$ theory $T[\mathrm{SU}(3)]$. Semi-squared nodes denote $3 \mathrm{~d}$ gauge symmetries gauging $1 \mathrm{~d}$ flavor symmetries.
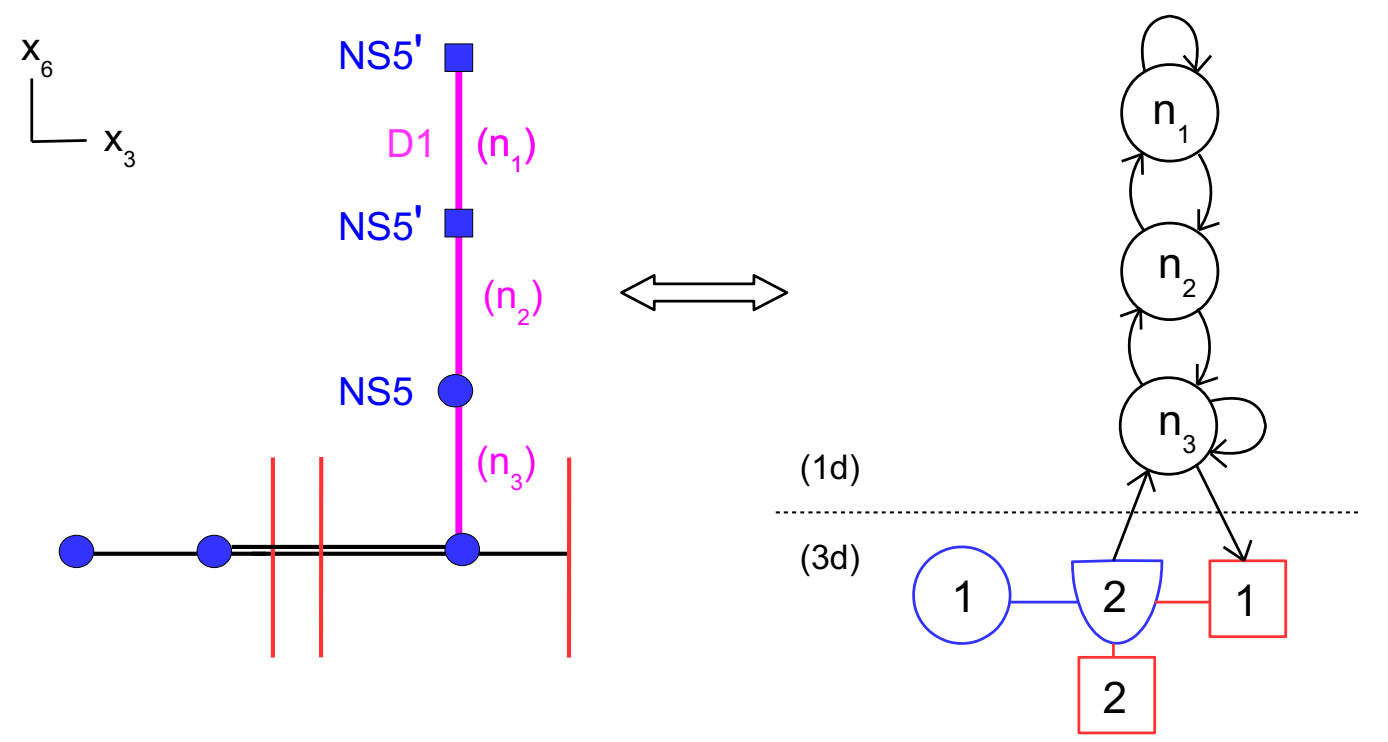

Figure 15. Pushing the D1-branes to the NS5-brane on the right, we can read another 3d/1d theory, giving a second UV realization of the same Vortex loop.

strings decreases, as in figure 16 . The initial $k$ strings split into $k=k_{1}+k_{2}$ strings, where $k_{1}$ strings end on the remaining D3-branes, while $k_{2}$ strings have been moved along the D3-spikes and end now on the D5, away from the main brane configuration. There are many possible final brane configurations, associated to the various choices of splittings (constrained by the s-rule) as D5-branes are moved across the F1-strings, and each of these final configuration realizes a Wilson loop in a certain node of the mirror theory, combined 


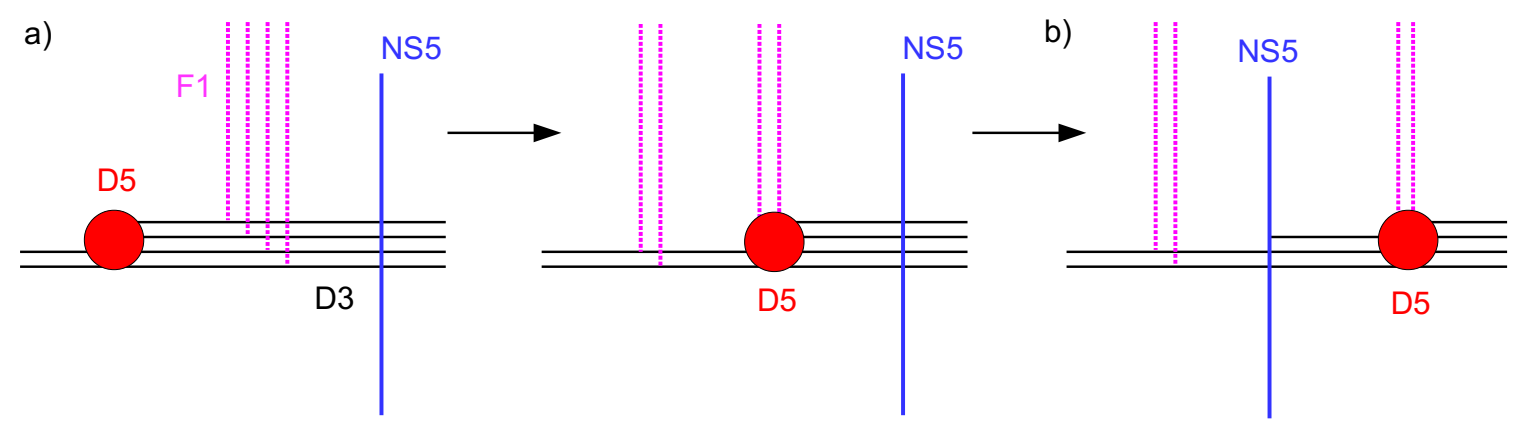

Figure 16. a) The D5-brane has to be moved to the right, across F1-strings, to reach the canonical brane configuration. b) After moving the D5-brane, stacks of F1-strings end on the D5-brane and are moved along transverse directions (transverse to the picture) away from the main brane configuration.

with flavor Wilson loops inserted by the strings ending on the D5-branes. Physically $q$ F1strings ending on a D5 insert a Wilson loop with charge $q$ under the U(1) flavor symmetry acting on the associated hypermultiplet. The mirror symmetry prediction is then that the initial Vortex loop is mapped to the sum of the Wilson loops realized by the possible final brane configurations. We will provide explicit mirror map predictions using this algorithm in the next section.

The simplest situations arise when no D5-branes must be moved across F1-strings, in which case the Vortex loop is mapped to a single Wilson loop in the mirror theory and both loops are labeled by the same representation $\mathcal{R}$ of a certain $\mathrm{U}(N)$ gauge group factor. This is the case for instance in circular quivers with nodes of equal rank, for which mirror symmetry is simply implemented by $S$-duality on the brane configuration and no D5-brane moves are required to reach the mirror dual configuration (see section 4.4).

In this section we have found a systematic algorithm to construct the mirror map between Wilson loop and Vortex loop operators in mirror dual theories. This can be applied to construct the mirror map for any pair of $3 \mathrm{~d} \mathcal{N}=4$ mirror quiver gauge theories of linear or circular type. We will apply this algorithm on several explicit examples below.

\section{Mirror symmetry and loop operators: examples}

In this section we give the explicit mirror map between loop operators using the ideas and tools introduced in the previous section. We provide examples both for linear and circular quivers.

We first discuss the action of mirror symmetry of loop operators for a class of $3 \mathrm{~d}$ $\mathcal{N}=4$ linear quivers that are self-mirror: that is $T[\mathrm{SU}(N)][29] . T[\mathrm{SU}(N)]$ is encoded by the quiver diagram of figure 17-a. The gauge group is $G=\prod_{j=1}^{N-1} \mathrm{U}(j)$ and there are $N$ fundamental hypermultiplets in the $\mathrm{U}(N-1)$ node. The brane realization of $T[\mathrm{SU}(N)]$ is shown in figure 17-b.

Constructing the mirror map for arbitrary loops in $T[\mathrm{SU}(N)]$ already incorporates all the subtleties and physical phenomena that emerge in the most general case already 
a)

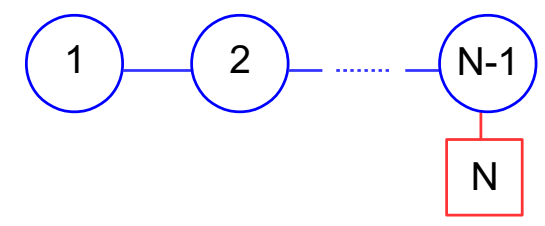

b)

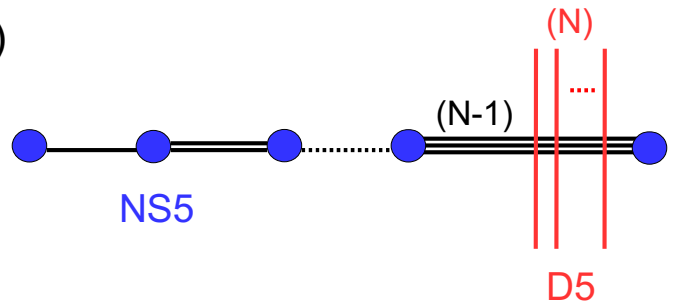

Figure 17. a) $T[\mathrm{SU}(N)]$ quiver. b) Brane realization.

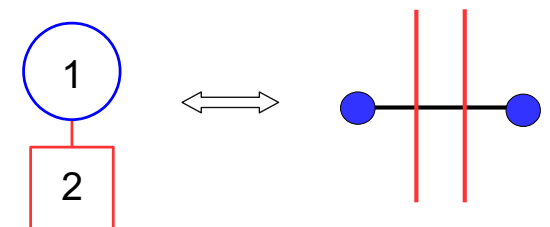

Figure 18. $T[\mathrm{SU}(2)]$ quiver and its brane realization.

discussed in section 3.2. We then provide duality maps for other examples of linear and circular quivers.

\section{$4.1 T[\mathrm{SU}(2)]$}

Consider a charge $k$ Wilson loop $W_{k}$ in $T[\mathrm{SU}(2)]$, a $\mathrm{U}(1)$ gauge theory with two fundamental hypermultiplets (see figure 18). The brane construction of $T[\mathrm{SU}(2)]$ has a single D3-brane stretched between two NS5-branes which is crossed by two D5-branes. According to the discussion of section 3.1, the insertion of the charge $k$ Wilson loop (which is the same as the $\mathcal{S}_{k}$ representation in an abelian theory) is realized by adding $k$ F1-strings stretched between the D3-brane and an extra D5-brane far away from the stack (figure 19-a). The F1-strings (and extra D5-brane) can be moved along the $x^{3}$ direction along the D3-brane without changing the infrared $3 \mathrm{~d}$ theory, so we can without loss of generality place the F1-strings between the two D5-branes.

Acting with S-duality on this enriched brane configuration and moving the D5-branes according to the Hanany-Witten rules we recover the same $T[\mathrm{SU}(2)]$ brane configuration but with $k$ D1-branes stretched between the D3-brane and an extra NS5-brane far away from the stack as in figure 19-c. The D1-branes end on the D3-brane between the two D5-branes. We note that this is the final S-dual brane configuration irrespectively of where we decide to place the F1-strings relative to the original D5-branes. The presence of the D1-branes is responsible for the insertion of a supersymmetric one-dimensional defect in the $T[\mathrm{SU}(2)]$ theory living on the D3-branes. We denote this defect by $V_{k}$.

The operator $V_{k}$ can be described by a $1 \mathrm{~d} \mathcal{N}=4$ gauge theory coupled to $T[\mathrm{SU}(2)]$. As explained above there are two alternative $3 \mathrm{~d} / 1 \mathrm{~d}$ defect descriptions of $V_{k}$, that we can read by moving the D1-branes on top of the nearest NS5-brane on the left or on the right. It is a non-trivial dynamical statement that these two descriptions of $V_{k}$ do indeed describe the same operator (see section 5). 

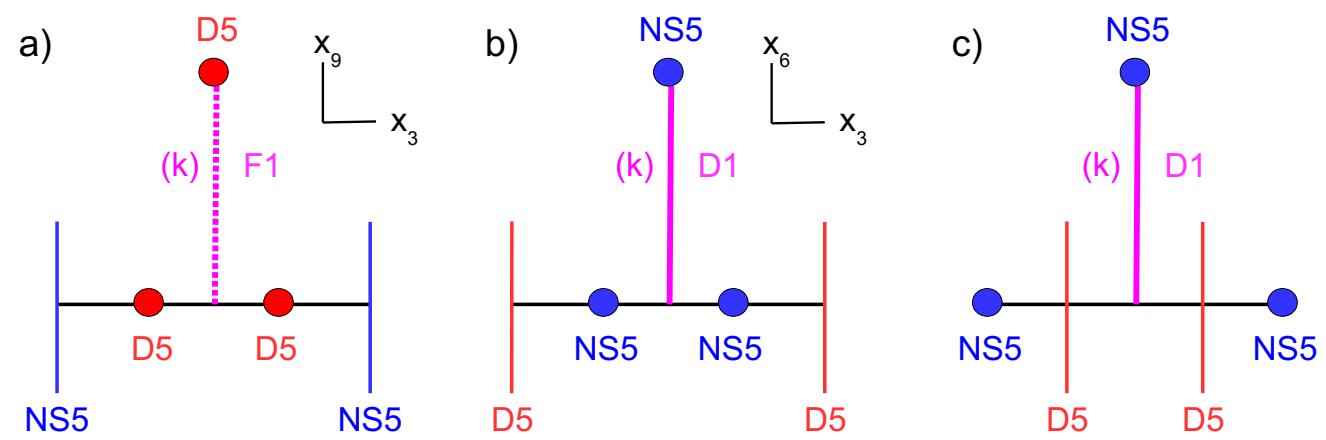

Figure 19. a) Brane realization of a charge $k$ Wilson loop insertion in $T[\mathrm{SU}(2)]$. b) Configuration after S-duality (and rotation). c) After Hanany-Witten D5-brane moves: $k$ D1-branes stretched between a NS5-brane and the D3-brane in the mirror $T[\mathrm{SU}(2)]$ brane configuration. The D1-branes end between the two D5-branes.

We start with the description of $V_{k}$ as a deformation of the theory where the D1-branes end on the left NS5-brane. This configuration is reached by first moving the left D5-brane to the left of the NS5-brane, creating a D3-brane by the Hanany-Witten effect, and then moving the D1-branes (together with the extra NS5-brane far away) until they end on the NS5-brane, thus reaching the configuration shown in figure 20. In the IR, the theory supported on the brane configuration is a $3 \mathrm{~d} / 1 \mathrm{~d} \mathcal{N}=4$ gauge theory coupled to $T[\mathrm{SU}(2)]$, which can be read from the rules described in the previous section. It is described by the $1 \mathrm{~d} \mathcal{N}=4$ quiver gauge theory of figure 20 , a $\mathrm{U}(k)$ gauge theory with an adjoint chiral multiplet, one fundamental and one anti-fundamental chiral multiplet coupled to $T[\mathrm{SU}(2)]$ as shown in figure 20.

The way the $1 \mathrm{~d} \mathcal{N}=4$ quiver gauge theory couples to $T[\mathrm{SU}(2)]$ can be read from the brane configuration. The $\mathrm{U}(1)^{-}$flavor symmetry acting on the anti-fundamental chiral multiplet is gauged with the $3 \mathrm{~d} \mathcal{N}=4 \mathrm{U}(1)$ vector multiplet, and the $\mathrm{U}(1)^{+}$flavor symmetry acting on the fundamental chiral multiplet is identified with the $\mathrm{U}(1)$ flavour symmetry acting on the $3 \mathrm{~d}$ hypermultiplet in $T[\mathrm{SU}(2)]$ associated to the left D5-brane. The operator $V_{k}$ is described by the coupled $3 \mathrm{~d} / 1 \mathrm{~d}$ theory summarized by the mixed $3 \mathrm{~d} / 1 \mathrm{~d}$ quiver diagram in 20. As explained in section 2, the identification of $1 \mathrm{~d}$ with $3 \mathrm{~d}$ flavour symmetries is implemented with a superpotential coupling (2.12) between the relevant defect chiral multiplets and bulk hypermultiplet. In the initial configuration (figure 19-c) the D1-branes end on the D3-branes instead of the left NS5-brane. The deformation corresponding to moving the D1-branes along the D3-brane to go back to the initial configuration corresponds to turning on an FI term with coupling $\eta \propto \Delta x^{3}>0$ in the SQM, where $\Delta x^{3}$ is the difference of the positions along $x^{3}$ between the left NS5-brane and the D1-branes.

Alternatively, we can describe the operator $V_{k}$ as a deformation of a configuration where the D1-branes end on the NS5-brane on the right. Following the same steps as before we reach the brane configuration of figure 21, and we find a description of the infrared loop operator $V_{k}$ as a different coupling of the same $1 \mathrm{~d} \mathcal{N}=4$ quiver gauge theory to $T[\mathrm{SU}(2)]$. Now the $\mathrm{U}(1)^{+}$flavor symmetry acting on the fundamental chiral multiplet is gauged with the $3 \mathrm{~d} \mathcal{N}=4 \mathrm{U}(1)$ vector multiplet of $T[\mathrm{SU}(2)]$, and the $\mathrm{U}(1)^{-}$flavor symmetry acting on 


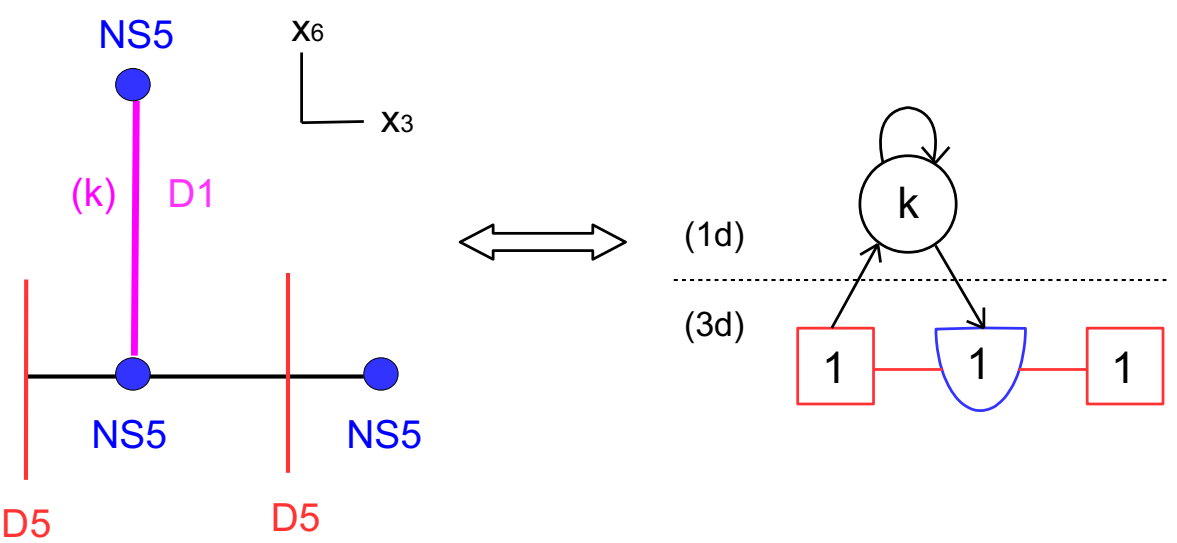

Figure 20. Brane configuration after moving the D1-branes on top of the left NS5-brane, as described in the text, and the quiver description of the $3 \mathrm{~d} / 1 \mathrm{~d}$ gauge theory. An arrow arriving to (resp. leaving) a node denotes a fundamental (resp. anti-fundamental) chiral multiplet for that node. This $3 \mathrm{~d} / 1 \mathrm{~d}$ defect theory describes the loop operator dual to the abelian Wilson loop in the mirror $T[\mathrm{SU}(2)]$ theory.

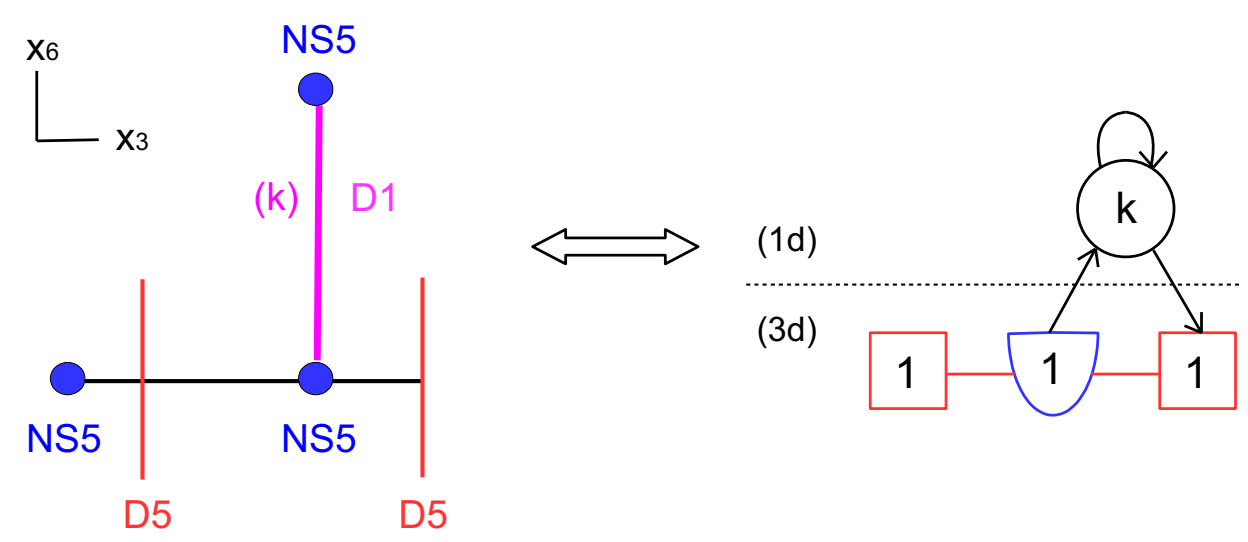

Figure 21. Alternative 3d/1d defect theory, read by moving the D1-branes to the right NS5-brane.

the anti-fundamental chiral multiplet is identified with the $\mathrm{U}(1)$ flavour symmetry acting on the $3 \mathrm{~d}$ hypermultiplet in $T[\mathrm{SU}(2)]$ associated to the right $\mathrm{D} 5$-brane. The operator $V_{k}$ is described by the coupled $3 \mathrm{~d} / 1 \mathrm{~d}$ theory summarized by the mixed $3 \mathrm{~d} / 1 \mathrm{~d}$ quiver diagram in 21. In the initial configuration (figure 19-c) the D1-branes end on the D3-branes instead of the right NS5-brane. The deformation corresponding to moving the D1-branes along the D3-brane to go back to the initial configuration corresponds to turning on a negative FI parameter $\eta \propto \Delta x^{3}<0$ in the SQM, where $\Delta x^{3}$ is the difference of the positions along $x^{3}$ between the right NS5-brane and the D1-branes.

We conclude that there are two different UV descriptions of the operator $V_{k}$ mirror dual to the charge $k$ Wilson loop $W_{k}$ in $T[\mathrm{SU}(2)]$. In section 5.6 we explicitly show that the exact expectation value of $V_{k}$ on $S^{3}$ computed using the two different UV definitions define the same operator and in section 5.4.1 that it matches the exact expectation value 
a)
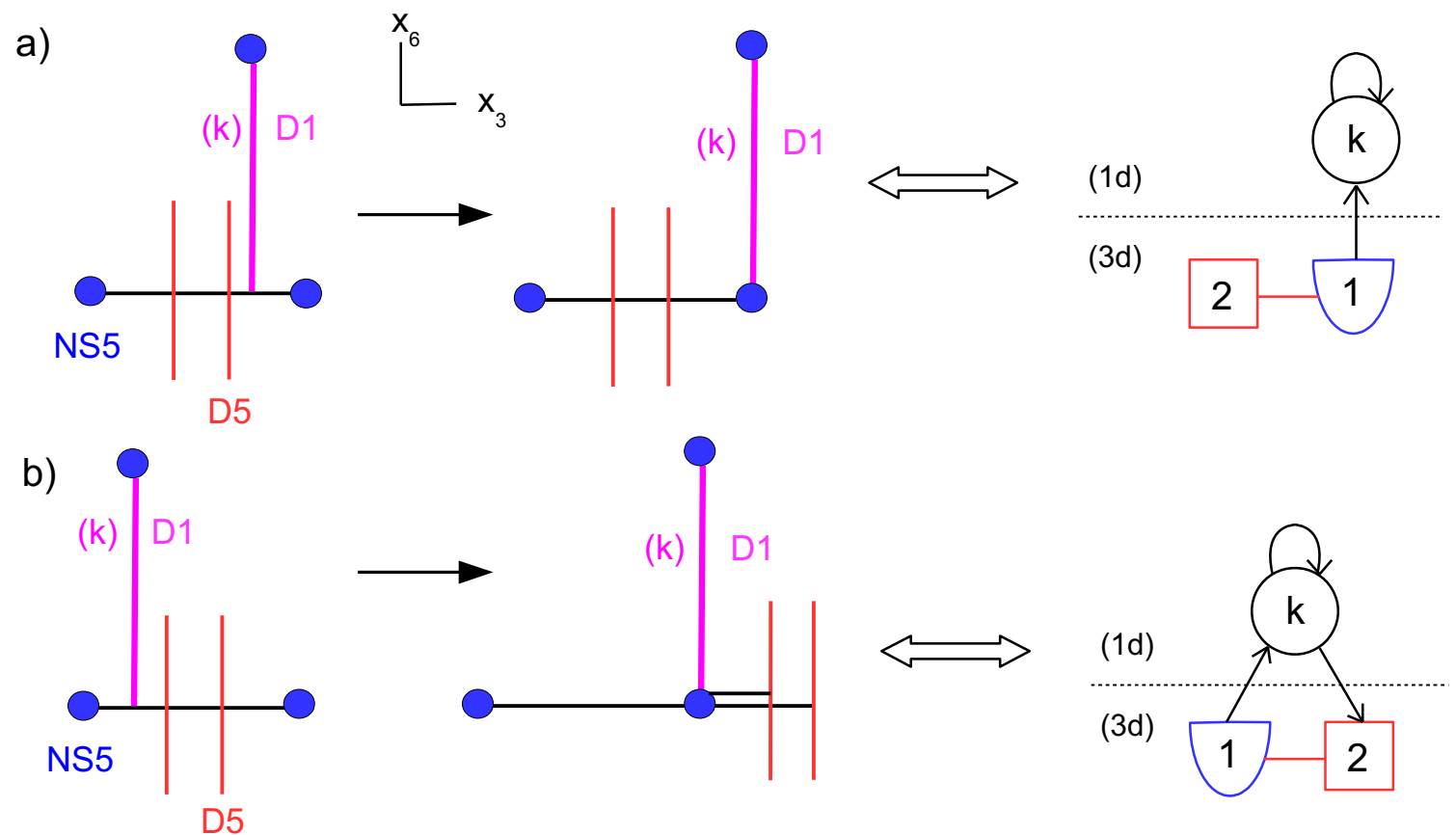

Figure 22. Brane realizations of other $T[\mathrm{SU}(2)]$ Vortex loops and associated $3 \mathrm{~d} / 1 \mathrm{~d}$ quiver theory read by moving the D1-branes to the right NS5-brane. a) Vortex loop realized with D1-branes placed to the right of the D5-branes. b) Vortex loop realized with D1-branes placed to the left of the D5-branes.

of $W_{k}$ under the exchange of mass and FI parameters

$$
\left\langle W_{k}\right\rangle \stackrel{\text { mirror }}{\longleftrightarrow}\left\langle V_{k}\right\rangle .
$$

We can also consider the Vortex loop operators realized by D1-branes ending on the D3-brane to the left or to the right of the two D5-branes. The corresponding 3d/1d quivers realizing these Vortex loops can be read by moving the D1-branes on top of the NS5-brane on the right, as shown in figure 22, or on the left. We analyze the mirror of such Vortex loop operators for the general $T[\mathrm{SU}(N)]$ theory in section 4.2. They are mirror to flavour Wilson loops (see discussion around equation (4.7)).

\subsection{Wilson loops in the $\mathrm{U}(N-1)$ node of $T[\mathrm{SU}(N)]$}

We now turn to Wilson loops in a non-abelian gauge theory. We start with Wilson loop operators in the $\mathrm{U}(N-1)$ node of the self-mirror $T[\mathrm{SU}(N)]$ theory. As we shall see, a Wilson loop for this (last) node maps directly to a single Vortex loop operator, described by a specific $3 \mathrm{~d} / 1 \mathrm{~d}$ gauge theory that we construct. Later we show that the expectation value of a Wilson loop operator in another node in $T[\mathrm{SU}(N)]$ maps under mirror symmetry to a specific combination of Vortex loop operators.

We start by considering a Wilson loop in the $k$-symmetric representation of $\mathrm{U}(N-1)$, which we denote by $W_{\mathcal{S}_{k}}^{(N-1)}$. The brane realization of this Wilson loop insertion comprises the D3-NS5-D5 system realizing the $T[\mathrm{SU}(N)]$ theory in figure 17 with $k$ additional F1strings stretched between the $N-1 \mathrm{D} 3$-branes supporting the $\mathrm{U}(N-1)$ node and an extra 
a)

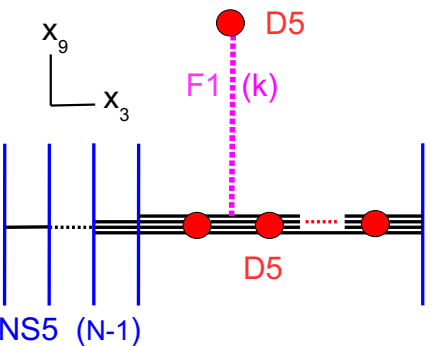

b)

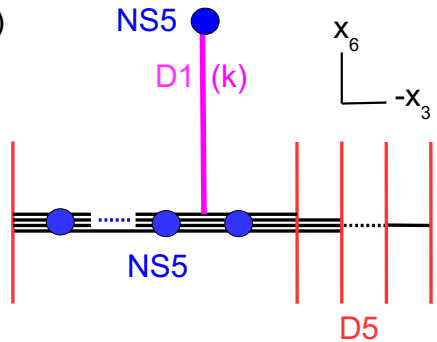

c)

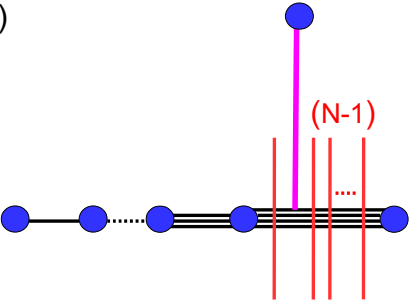

Figure 23. a) Brane configuration realizing a $\mathcal{S}_{k}$ Wilson loop in $T[\mathrm{SU}(N)]$. b) S-dual brane configuration, shown in a different orientation (in particular the $x^{3}$ orientation is reversed). c) Brane configuration after D5-brane rearranging, realizing a 3d/1d defect theory.

D5-brane away from the stack, as depicted in figure 23-a. We are free to place the F1strings anywhere relative to the $N$ D5-branes that intersect the D3-branes, as this results in the same Wilson loop operator in the IR.

The mirror dual loop operator to $W_{\mathcal{S}_{k}}^{(N-1)}$ is obtained by analyzing the S-dual configuration (figure 23-b), where we have reversed the $x^{3}$ direction for convenience. ${ }^{47}$ First we move the D5-branes in the direction of D3-brane excess to reach the $T[\mathrm{SU}(N)]$ brane configuration, now enriched with $k$ D1-branes stretched between the $N-1$ D3-branes associated to the $\mathrm{U}(N-1)$ node and an extra NS5-brane far away from the stack (see figure 23-c). The D1-branes are positioned with one D5 to their left and $N-1$ D5-branes to their right. This final configuration is the same irrespective of where the original F1strings were placed relative to the D5-branes. In order to reach this configuration we are careful not to let D5-branes cross the D1-branes in the rearrangement, since this would affect the infrared theory living on the D1-branes. This is S-dual to the statement that we do not let F1-strings move across NS5-branes, as this clearly changes the Wilson loop inserted. The position of the D1-branes relative to the D5-branes is crucial in determining the Vortex loop operator just as the position of the F1-strings relative to the NS5-branes is crucial in determining the Wilson loop operator. On the other hand, we let the D1-branes move freely across an NS5-brane when the number of D3-branes is the same on both sides of the NS5-brane. This is S-dual to the fact that F1-strings can be freely moved across D5-branes when the number of D3-branes is the same on both sides of the D5-branes. We denote the loop operator defined by the S-dual brane configuration by $V_{N-1, \mathcal{S}_{k}}$, where the subscript $N-1$ encodes the number of D5-branes to the right of the D1-branes.

The coupled $3 \mathrm{~d} / 1 \mathrm{~d}$ theory living on the final brane configuration can be read again in two ways, depending on which of the nearby NS5-branes we let the D1-branes end, as explained in section 3.2. One possibility is to move the $N-1 \mathrm{D} 5$-branes to the right and let the D1-branes end on the NS5-brane on their right as shown in figure 24-a. The associated $1 \mathrm{~d} \mathcal{N}=4$ gauge theory is a $\mathrm{U}(k)$ vector multiplet, with an adjoint chiral multiplet, $N-1$ fundamental and $N-1$ anti-fundamental chiral multiplets. This $1 \mathrm{~d} \mathcal{N}=4$ theory is captured by the $1 \mathrm{~d}$ part of quiver diagram in 24-a.

\footnotetext{
${ }^{47}$ This extra reflection allows us to bring the branes (in the absence of F1 or D1) back to their initial configuration, showing that the theory $T[\mathrm{SU}(N)]$ is self-mirror.
} 

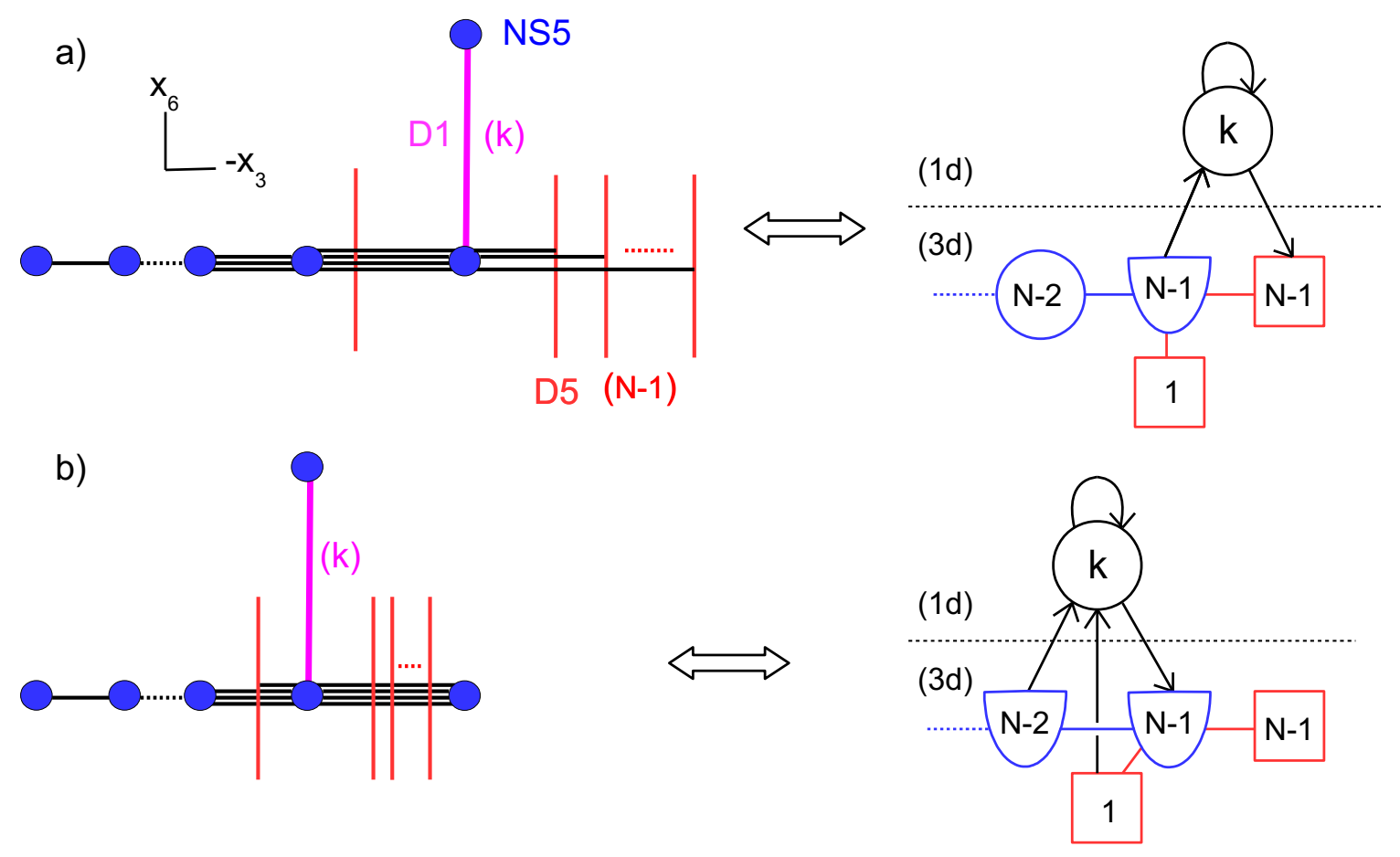

Figure 24. a) Brane configuration with D1-branes ending on the right NS5 and associated 3d/1d defect gauge theory. b) Brane configuration with D1-branes ending on the left NS5-brane and associated dual defect theory.

The way the $1 \mathrm{~d}$ gauge theory couples to the $3 \mathrm{~d} T[\mathrm{SU}(N)]$ theory is also captured by the brane configuration in figure 24 -a. The $\mathrm{U}(N-1)$ flavour symmetry rotating the fundamental chirals is gauged with the $3 \mathrm{~d} \mathrm{U}(N-1)$ vector multiplet symmetry and the $\mathrm{U}(N-1)$ symmetry rotating the anti-fundamental chirals is identified with the $\mathrm{U}(N-1)$ flavor symmetry acting on the $3 \mathrm{~d}$ hypermultiplets associated to the $N-1$ D5-branes. In order to describe the actual operator $V_{N-1, \mathcal{S}_{k}}$ the $1 \mathrm{~d} \mathcal{N}=4$ gauge theory must be deformed with a negative FI parameter. The coupled $3 \mathrm{~d} / 1 \mathrm{~d}$ description of the Vortex loop operator $V_{N-1, \mathcal{S}_{k}}$ mirror to $W_{\mathcal{S}_{k}}^{(N-1)}$ is captured by the quiver diagram in figure 24-a.

Another description of $V_{N-1, \mathcal{S}_{k}}$ can also be read from the configuration where the D1-branes end on the nearest NS5-brane to their left, with the (single) leftmost D5-brane pushed to the left of that NS5-brane. The $1 \mathrm{~d} \mathcal{N}=4$ gauge theory living on the D1-branes is the same as before, but the way it couples to $T[\mathrm{SU}(N)]$ is rather different. The $\mathrm{U}(N-1)$ flavour symmetry rotating the anti-fundamental chirals is gauged with the $3 \mathrm{~d} \mathrm{U}(N-1)$ vector multiplet. The $\mathrm{U}(N-1)$ flavour symmetry rotating the fundamental chirals is broken to $\mathrm{U}(N-2) \times \mathrm{U}(1)$. The $\mathrm{U}(N-2)$ flavour symmetry is gauged with the $3 \mathrm{~d} \mathrm{U}(N-2)$ vector multiplet and the remaining $\mathrm{U}(1)$ flavour symmetry is identified with the $\mathrm{U}(1)$ flavor symmetry acting on the 3 d hypermultiplet associated to the leftmost D5-brane. Now, the $1 \mathrm{~d} \mathcal{N}=4$ gauge theory has to be deformed with a positive FI parameter as the D1-branes end to the right of the reference NS5-brane. The coupled 3d/1d description of the Vortex loop operator $V_{N-1, \mathcal{S}_{k}}$ mirror to $W_{\mathcal{S}_{k}}^{(N-1)}$ is captured by the quiver diagram in figure 24-b. 

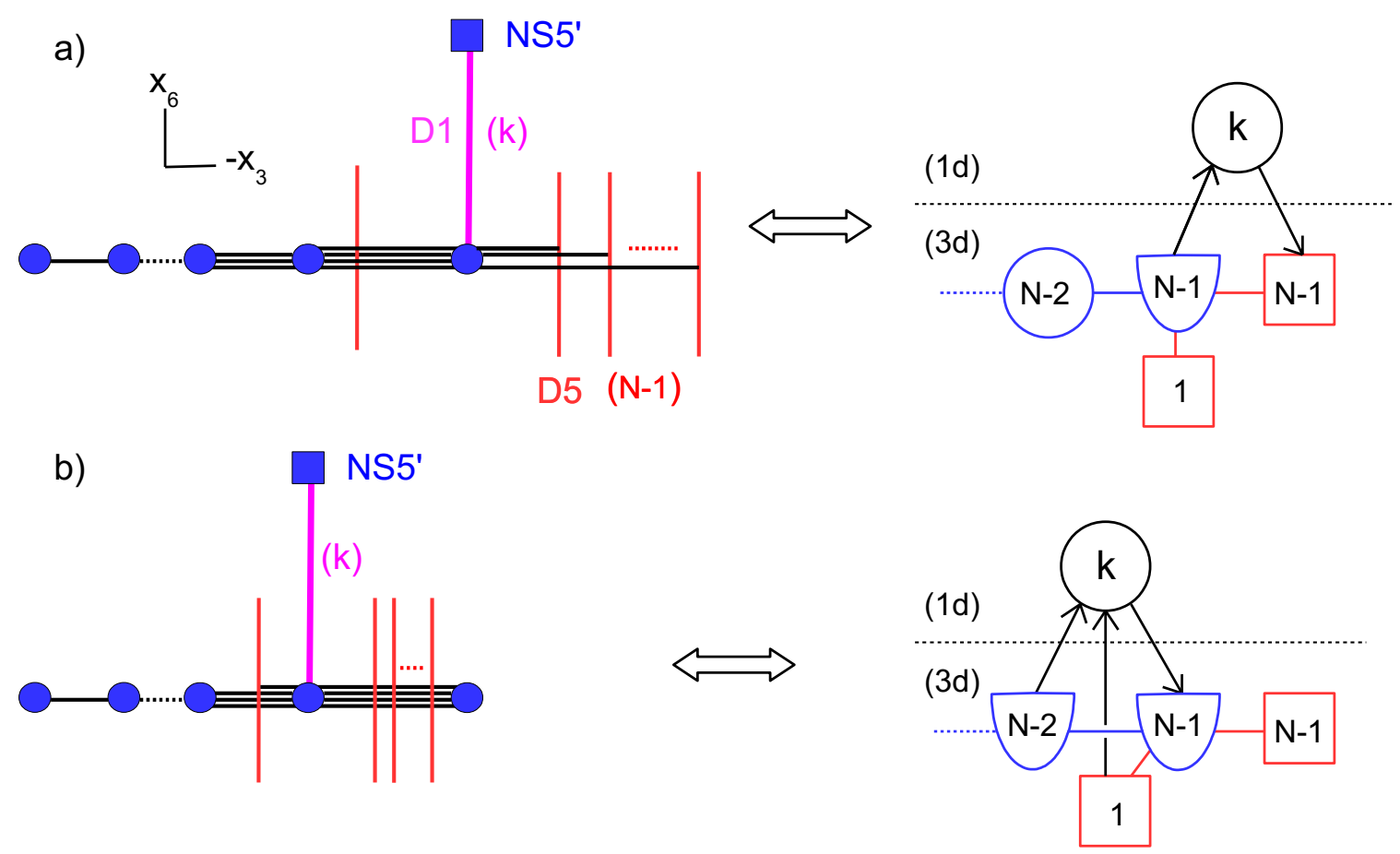

Figure 25. Brane configuration with $k$ D1-strings ending on a single D5'-brane and associated left and right $3 \mathrm{~d} / 1 \mathrm{~d}$ quivers.

In summary, we obtain the following mirror map of loop operators in $T[\mathrm{SU}(N)]$ theory

$$
\left\langle W_{\mathcal{S}_{k}}^{(N-1)}\right\rangle \stackrel{\text { mirror }}{\longleftrightarrow}\left\langle V_{N-1, \mathcal{S}_{k}}\right\rangle
$$

The situation is almost the same if we start with a Wilson loop in the $k$-antisymmetric representation of $\mathrm{U}(N-1)$, which we denote by $W_{\mathcal{A}_{k}}^{(N-1)}$. In this case the Wilson loop in realized with $k$ F1-strings stretched between an extra D5'-brane and the $N-1$ D3branes. After S-duality and brane rearranging we obtain the same brane configuration as in figure 23-c, except that D1-branes end on an NS5'-brane instead of a NS5-brane. The two possible $3 \mathrm{~d} / 1 \mathrm{~d}$ defect theories describing the mirror loop operator, which we denote by $V_{N-1, \mathcal{A}_{k}}$, are given in figure 25 . They are the same as in figure 24, except that there is no adjoint chiral multiplet in the SQM. In this case we get the following duality map

$$
\left\langle W_{\mathcal{A}_{k}}^{(N-1)}\right\rangle \stackrel{\text { mirror }}{\longleftrightarrow}\left\langle V_{N-1, \mathcal{A}_{k}}\right\rangle \text {. }
$$

In section 5.4 we explicitly show by computing the exact expectation value of these loop operators on $S^{3}$ that (4.2) and (4.3) hold under the exchange of mass and FI parameters.

The case of the Wilson loop in a tensor product of symmetric and anti-symmetric representations $\mathcal{R}=\otimes_{a=1}^{d} \mathcal{S}_{k^{(a)}} \otimes_{b=1}^{d^{\prime}} \mathcal{A}_{l^{(b)}}$ of $\mathrm{U}(N-1)$ in $T[\mathrm{SU}(N)]$ is analogous. We denote such a Wilson loop by $W_{\mathcal{R}}^{(N-1)}$. As explained in section 3.2, the Wilson loop insertion is realized with a collection of $d$ D5-branes and $d^{\prime}$ D5'-branes, with $k^{(a)}$ F1strings suspended between the $N-1$ D3-branes and the $a$-th D5-brane, and $l^{(b)}$ F1-strings suspended between the $N-1$ D3-branes and the $b$-th D5'-brane. The case $d=d^{\prime}=2$ is displayed in figure 26-a. After S-duality and D5-brane moves, we obtain a configuration 
a)

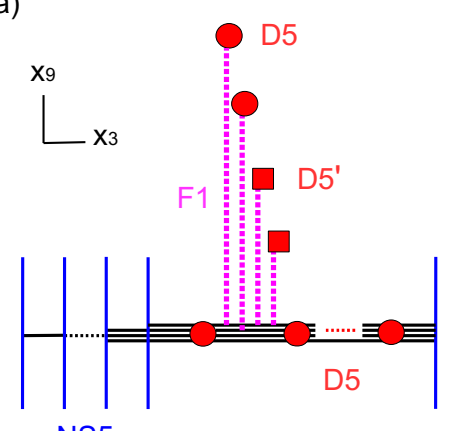

NS5 b)

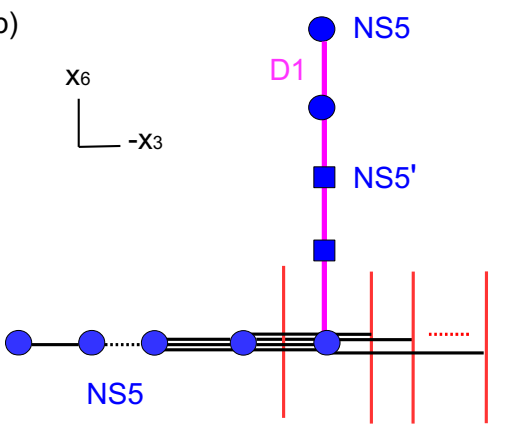

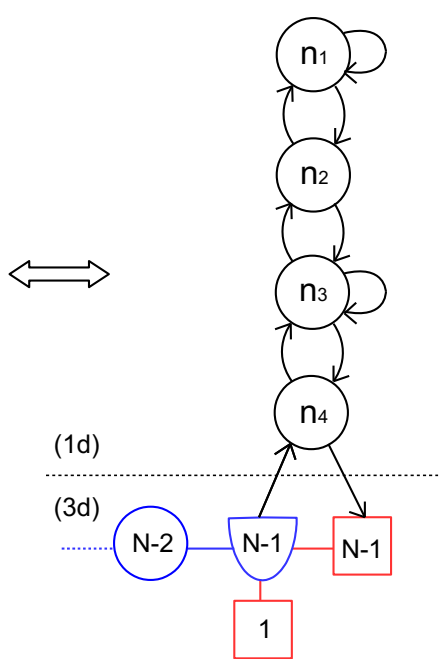

Figure 26. a) Brane configuration inserting a Wilson loop in the $\mathcal{S}_{k^{(1)}} \otimes \mathcal{S}_{k^{(2)}} \otimes \mathcal{A}_{l^{(1)}} \otimes \mathcal{A}_{l^{(2)}}$ representation of $\mathrm{U}(N-1)$. b) S-dual brane configuration with D1-branes ending on the right NS5-brane and associated dual 3d/1d defect theory with $\mathrm{U}\left(n_{1}\right) \times \mathrm{U}\left(n_{2}\right) \times \mathrm{U}\left(n_{3}\right) \times \mathrm{U}\left(n_{4}\right)$ SQM. The ranks are $n_{1}=k^{(1)}, n_{2}=k^{(1)}+k^{(2)}, n_{3}=k^{(1)}+k^{(2)}+l^{(1)}, n_{4}=k^{(1)}+k^{(2)}+l^{(1)}+l^{(2)}$.

with $d$ NS5-branes and $d^{\prime}$ NS5'-branes, with $k^{(a)}$ D1-branes suspended between the $N-1$ D3-branes and the $a$-th NS5-brane, and with $l^{(b)}$ D1-branes suspended between the $N-1$ D3-branes and the $b$-th NS5'-brane, realizing the mirror dual loop operator, which we denote by $V_{N-1, \mathcal{R}}$. In the initial configuration, the relative positions of the D5-branes and D5'-branes in the $x^{9}$ direction were irrelevant for the insertion of the Wilson loop operator. In the S-dual picture, the positions of the NS5-branes and NS5'-branes along the $x^{6}$ direction are important, since they affect the $1 \mathrm{~d} \mathcal{N}=4$ gauge theory living on the D1branes. The brane picture, however, implies that all the possible $3 \mathrm{~d} / 1 \mathrm{~d}$ defect field theories obtained from picking different orderings of the NS5 and NS5' brane positions along $x^{6}$ direction are equivalent/dual descriptions of the loop operator $V_{N-1, \mathcal{R}}$. In section 5.3.2 we provide some explicit evidence that these are indeed dual descriptions of the same operator by computing the exact partition function on $S^{3}$ of the $3 \mathrm{~d} / 1 \mathrm{~d}$ defect theories capturing $\left\langle V_{N-1, \mathcal{R}}\right\rangle$ and showing that they are the same.

The $3 \mathrm{~d} / 1 \mathrm{~d}$ defect theories describing the resulting Vortex loop $V_{N-1, \mathcal{R}}$ can be read from the rules of section 3.2 by moving the D1-branes to the closest NS5 on the left or on the right. The example of the Vortex loop associated to the representation $\mathcal{R}=$ $\mathcal{S}_{k^{(1)}} \otimes \mathcal{S}_{k^{(2)}} \otimes \mathcal{A}_{l^{(1)}} \otimes \mathcal{A}_{l^{(2)}}$ with a specific ordering of the NS5/NS5'-branes along $x^{6}$ is worked out in figure 26-b.

In summary, we obtain the following mirror map of those loop operators in $T[\mathrm{SU}(N)]$ theory:

$$
\left\langle W_{\mathcal{R}}^{(N-1)}\right\rangle \stackrel{\text { mirror }}{\longleftrightarrow}\left\langle V_{N-1, \mathcal{R}}\right\rangle .
$$

We have found that the mirror of a Wilson loop labeled by a representation of the $\mathrm{U}(N-1)$ node is a Vortex loop operator labeled by the same representation. We now turn to the more involved case of a Wilson loop in a representation of another gauge group factor in $T[\mathrm{SU}(N)]$. 


\subsection{The other $T[\mathrm{SU}(N)]$ loops}

We proceed now with constructing the mirror map for the remaining loop operators in $T[\mathrm{SU}(N)]$. This includes the Wilson loop operators in the other nodes of $T[\mathrm{SU}(N)]$. As explained in section 3.2 it is easier to describe the mirror map starting from the brane configuration realizing Vortex loops and going to the S-dual picture with F1-strings realizing the mirror Wilson loops. In the brane realization, defining a Wilson loop requires specifying the location of the F1-strings relative to the NS5-branes and a representation $\mathcal{R}$. Likewise, a Vortex loop is specified by the location of the D1-branes relative to the D5-branes and by a choice of representation $\mathcal{R}$ of the gauge group supported on the D3-branes where the D1-branes end.

In the $T[\mathrm{SU}(N)]$ theory we have Vortex loops labeled by a representation $\mathcal{R}$ of the $\mathrm{U}(N-1)$ gauge node, which are realized with D1-strings ending on the $N-1$ D3-branes supporting that gauge group factor. As just discussed, the relative position of the D1-branes along the $x^{3}$ direction relative to the D5-branes is important. In the brane realization of $T[\mathrm{SU}(N)]$, there are $N$ D5-branes crossing the $N-1 \mathrm{D} 3$-branes, giving rise to the $N$ fundamental hypermultiplets of the $\mathrm{U}(N-1)$ node. Each $\mathrm{U}(N-1)$ Vortex loop is characterized by a splitting of the $N$ hypermultiplets into $N=M+(N-M)$ and corresponds to the arrangement where the D1-branes have $M$ D5-branes to their right and $N-M$ D5-branes to their left. Thus, the data characterizing a $\mathrm{U}(N-1)$ Vortex loop is a representation $\mathcal{R}$ of $\mathrm{U}(N-1)$ and an integer $0 \leq M \leq N$. We denote such a Vortex loop by $V_{M, \mathcal{R}}$. These operators admit a description in terms of a $1 \mathrm{~d} \mathcal{N}=4$ quiver gauge theory coupled to $T[\mathrm{SU}(N)]$, which is shown in figure 27-a for the case when the the D1-branes are moved to be on top of the closest NS5-brane to their right. As already explained in section 4.2 the Vortex loop operator $V_{N-1, \mathcal{R}}$ gets mapped under mirror symmetry to a Wilson loop $W_{\mathcal{R}}^{(N-1)}$ in a representation $\mathcal{R}$ of $\mathrm{U}(N-1)$.

This does not exhaust the Vortex loops that can be defined in $T[\mathrm{SU}(N)]$. There are Vortex loop operators in $T[\mathrm{SU}(N)]$ realized by D1-strings ending on the $p$ D3-branes supporting the $\mathrm{U}(p)$ gauge group factor, with $1 \leq p \leq N-2$. Since there are no fundamental hypermultiplets for this gauge group factor, and correspondingly no D5-branes crossing the D3-branes supporting the $\mathrm{U}(p)$ gauge group factor, Vortex loop operators in a $\mathrm{U}(p)$ node are simply labelled by a representation $\mathcal{R}$ of $\mathrm{U}(p)$. We denote them by $V_{\mathcal{R}}^{(p)}$, with $1 \leq p \leq N-2$. These operators admit a description in terms of a $1 \mathrm{~d} \mathcal{N}=4$ quiver gauge theory coupled to $T[\mathrm{SU}(N)]$, which is shown in figure 27-b for the case when the D1-branes are moved to be on top of the closest NS5-brane to their left.

Let us start by considering a Vortex loop operators $V_{M, \mathcal{R}}$ with $0 \leq M \leq N-1$. The case $V_{N, \mathcal{R}}$ will be analyzed when discussing the Vortex loop operators $V_{\mathcal{R}}^{(p)}$, which whom they share a similar physical mirror interpretation. An operator $V_{M, \mathcal{R}}$ is realized by $k$ D1branes with $N-M$ D5-branes on their left and $M$ D5-branes on their right and ending on the $N-1 \mathrm{D} 3$-branes supporting the $\mathrm{U}(N-1)$ gauge node on one end and on a number of NS5 and NS5'-branes on the other end. $k$ denotes the total number of boxes in the Young tableau associated to the representation $\mathcal{R}$, which we also denote by $|\mathcal{R}|$. Finding the mirror loop operator requires performing S-duality on the brane configuration (followed by 
a)

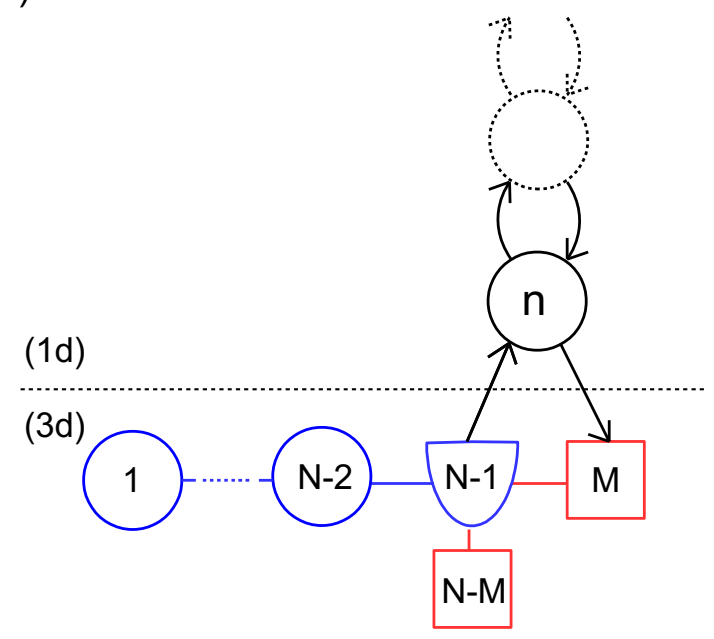

b)

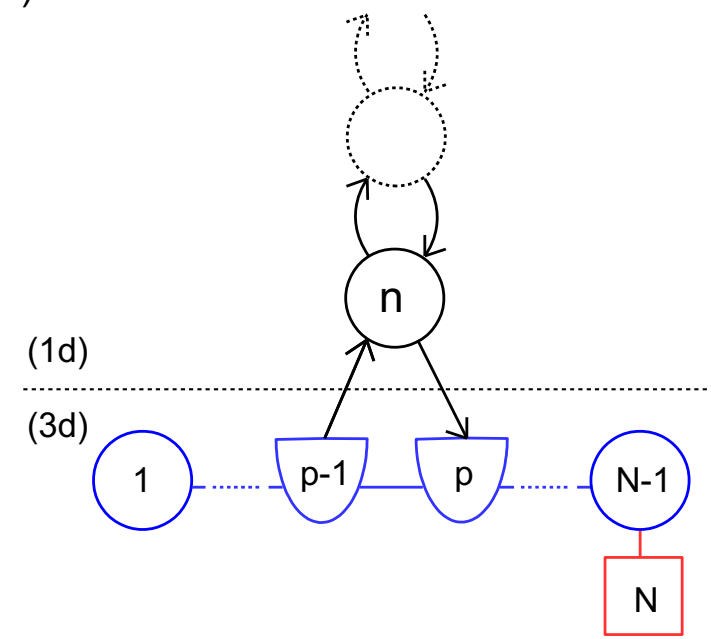

Figure 27. a) 3d/1d quiver description of Vortex loop $V_{M, \mathcal{R}}$. Here it is derived by moving the D1-strings to the right NS5-brane. b) 3d/1d quiver description of Vortex loop $V_{\mathcal{R}}^{(p)}$, derived by moving the D1-strings to the left NS5-brane.

a left-right reflection). The resulting configuration with F1-strings is shown in figure 28-b. In order to reach the canonical brane configuration of the mirror $T[\operatorname{SU}(N)]$ theory, one has to move the D5-branes until they all lie between the two rightmost NS5-branes. However, except for the configuration realizing $V_{N-1, \mathcal{R}}$ studied in the previous section, a D5-brane (the leftmost one) has to cross the F1-strings in this process. After this exchange, the number of D3-branes at the bottom of the F1-stack decreases. As explained earlier, when a D5-brane crosses the stack of $k$ F1-strings, $q \leq k$ of them can be moved smoothly from ending on the D3-branes to ending on the D5-brane. Intuitively this is possible since the D5-brane has a D3-brane spike and the F1-strings can be moved smoothly far from the main brane configuration along this spike. In the final brane picture shown in figure 28-c for $0 \leq M \leq N-1$, the $k$ original F1-strings are split into $\hat{k}$ strings ending on the $M$ D3-branes supporting the $\mathrm{U}(M)$ gauge group factor and $q$ strings ending on the leftmost D5-brane, such that $k=\hat{k}+q \cdot{ }^{48}$ Such a brane configuration realizes a Wilson loop in a representation $\widehat{\mathcal{R}}$ of $\mathrm{U}(M)$, which we denote by $W_{\widehat{\mathcal{R}}}^{(M)}$, where $|\widehat{\mathcal{R}}|=\hat{k}$, together with a Wilson loop of charge $q$ under the $\mathrm{U}(1)$ flavor symmetry rotating the $N$-th hypermultiplet (associated to the leftmost D5-brane), that we denote by $W_{N, q}^{\mathrm{fl}}$.

There are multiple ways of partitioning the $k$ F1-strings into $\hat{k}$ strings ending on the D3-branes and $\hat{q}$ F1-branes attached to the leftmost D5-brane. We propose that the mirror of $V_{M, \mathcal{R}}$ with $0 \leq M \leq N-1$ is obtained by summing over the Wilson loops of the mirror dual theory realized by all the possible final brane configurations consistent with the s-rule.

Our remaining task is to determine the precise sum over Wilson loops $W_{\widehat{\mathcal{R}}}^{(M)}$ and the representations $\widehat{\mathcal{R}}$ of $\mathrm{U}(M)$ that appear in the mirror of $V_{M, \mathcal{R}}$. In section 3.1 we considered

\footnotetext{
${ }^{48}$ The orientation of the F1-strings stretched between the D5/D5' away from the main stack and the leftmost D5 seem to break the supersymmetry of the configuration, however this is not the case in the limit when they are sent far away along the transverse $x^{4,5,6}$ directions.
} 
a) $\quad \mathrm{X}_{6}$

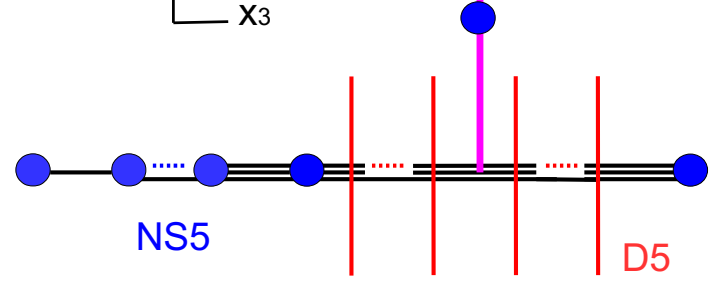

c)

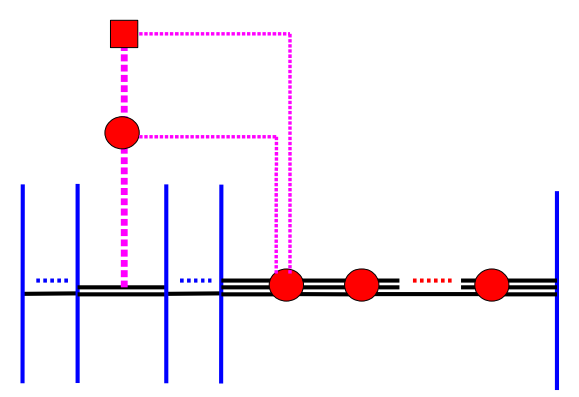

b)

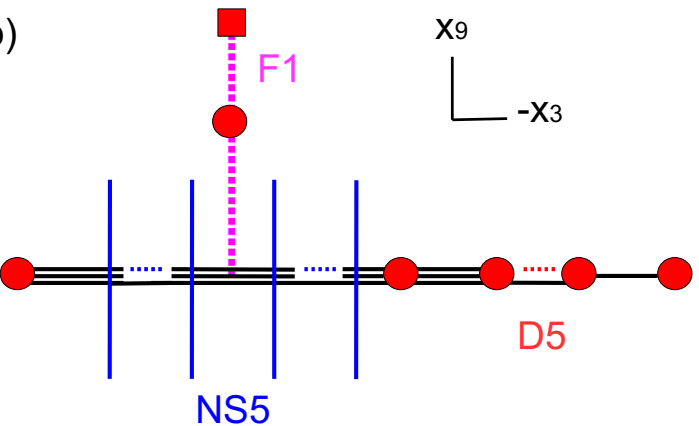

Figure 28. a) Brane configuration inserting a Vortex loop $V_{M, \mathcal{R}}$. b) S-dual brane configuration with F1-strings. c) Configuration after moving the D5-branes. Straight strings, ending on D3-branes realize Wilson loops. The other strings, which are also straight despite their pictorial representation, ending on the left D5-brane, are away from the main configuration and realize flavor Wilson loops.

brane configurations realizing Wilson loops with F1-strings ending on D3-branes on one end and on a set of D5 and D5'-branes on the other end. We argued that the set of patterns of F1-strings ending on $N$ D3-branes are in one-to one correspondence with the weights of a representation of $\mathrm{U}(N)$ associated to the Wilson loop realized by the brane configuration. The same identification holds for D1-branes ending on D3-branes realizing a Vortex loop. More explicitly, a given weight $w=\left(w_{1}, w_{2}, \cdots, w_{N}\right)$ corresponds to a pattern of strings (both for F1 and D1's) where $w_{i}$ strings end on the $i$-th D3-brane in the stack. The expectation value of a loop operator, irrespective of whether it is a Wilson loop or a Vortex loop, is obtained by summing over all weights, including degeneracies, which in the case of the Vortex loop are in one-to-one correspondence with the vacua of the corresponding $1 \mathrm{~d} \mathcal{N}=4 \mathrm{SQM}$ that realizes the Vortex loop. In the brane language, this means summing over the string patterns allowed by the brane realization. This physical picture will be made rather explicit in section 5.3 when we evaluate the exact $1 \mathrm{~d} \mathcal{N}=4 \mathrm{SQM}$ partition function describing a Vortex loop.

After these preliminaries we can now keep track of what happens to a single-weight pattern for the Vortex loop $V_{M, \mathcal{R}}$ associated to a weight $w=\left(w_{1}, w_{2}, \cdots, w_{N-1}\right)$, where $w_{j}$ D1-strings end on the $j$-th D3-brane, as we go to the mirror dual brane configuration. Implementing $S$-duality and moving the D5-branes as explained, we find the situation where the leftmost D5-brane crosses the stack of F1-strings. A total of $N-1-M$ D3-branes at the end of the F1-strings disappear in the process of exchanging the branes, and the F1- 
strings that are ending on them get attached to the D5-brane. Labelling the D3-branes that disappear by $j=M+1, \cdots, N-1$, we find that a total of $q=w_{M+1}+w_{M+2}+\cdots+w_{N-1}$ F1-strings get attached to the D5-brane. Therefore, the final brane configuration realizes a charge $q \mathrm{U}(1)$ flavor Wilson loop together with a $\hat{w}=\left(w_{1}, w_{2}, \cdots, w_{M}\right)$ single-weight contribution to a Wilson loop associated to the remaining F1-strings ending on the $M$ D3branes supporting the $\mathrm{U}(M)$ gauge node. Enumerating all the final patterns associated to all the weights $w$ of $\mathcal{R}$, we find that they realize the weights of all the representations $(\mathbf{q}, \widehat{\mathcal{R}})$, counted with degeneracies, appearing in the decomposition of the representation $\mathcal{R}$ of $\mathrm{U}(N)$ under the subgroup $\mathrm{U}(1) \times \mathrm{U}(M)$, where $\mathrm{U}(M)$ is embedded as $\mathrm{U}(M) \times \mathrm{U}(N-M-1) \subset$ $\mathrm{U}(N-1)$ and $\mathrm{U}(1)$ is embedded diagonally in $\mathrm{U}(N-M-1)$.

Our mirror symmetry prediction is therefore that the vortex loop $V_{M, \mathcal{R}}$ gets mapped to the sum of combined gauge and flavor Wilson loops $W_{N, \mathbf{q}}^{\mathrm{fl}} W_{\widehat{\mathcal{R}}}^{(M)}$, with the sum running over the representations $(\mathbf{q}, \widehat{\mathcal{R}})$ appearing in the decomposition of the representation $\mathcal{R}$ under the subgroup $\mathrm{U}(1) \times \mathrm{U}(M)$ of $\mathrm{U}(N-1)$, where $\mathrm{U}(M)$ is embedded as $\mathrm{U}(M) \times \mathrm{U}(N-M-1) \subset$ $\mathrm{U}(N-1)$ and $\mathrm{U}(1)$ is embedded diagonally in $\mathrm{U}(N-M-1)$. In formulas

$$
\begin{aligned}
\mathrm{U}(N-1) & \rightarrow \mathrm{U}(1) \times \mathrm{U}(M) \\
\mathcal{R} & \rightarrow \bigoplus_{s \in \Delta_{M}}\left(\mathbf{q}_{s}, \widehat{\mathcal{R}}_{s}\right),
\end{aligned}
$$

where $\Delta_{M}$ denotes the set of representations $(\mathbf{q}, \widehat{\mathcal{R}})$ in the decomposition of $\mathcal{R}$ counted with degeneracies. Our mirror map is therefore

$$
\left\langle V_{M, \mathcal{R}}\right\rangle \stackrel{\text { mirror }}{\longleftrightarrow}\left\langle\sum_{s \in \Delta_{M}} W_{N, \mathbf{q}_{s}}^{\mathrm{fl}} W_{\widehat{\mathcal{R}}_{s}}^{\mathrm{U}(M)}\right\rangle \quad 0 \leq M \leq N-1,
$$

Pleasingly, this map is reproduced in examples in the exact computations of section 5.4. ${ }^{49}$ The Vortex loop $V_{0, \mathcal{R}}$ is special: it is mapped to a pure flavor Wilson loop.

Let us now conclude with the mirror map for the Vortex loops $V_{\mathcal{R}}^{(p)}$ and $V_{N, \mathcal{R}}$, which is qualitatively different to the one for $V_{M, \mathcal{R}}$ with $0 \leq M \leq N-1$. Let us consider the Vortex loop $V_{\mathcal{R}}^{(p)}$, which is realized with $k$ D1-branes ending on the $p$ D3-branes supporting the $\mathrm{U}(p)$ gauge node on one end and on a number of NS5 and NS5'-branes on the other, as shown in figure 29-a. Here $k=|\mathcal{R}|$ is again the total number of boxes in the Young tableau associated to the representation $\mathcal{R}$ of $\mathrm{U}(p)$. In order to find the mirror loop we perform once again S-duality on this brane configuration (together with an $x^{3}$ reflection). The resulting configuration with F1-strings is shown in figure 29-b. In order to reach the canonical brane configuration of the mirror $T[\mathrm{SU}(N)]$ theory, one has to move the D5-branes. In the process $p$ out the $N$ D5-branes have to cross the stack of F1-strings from the right, after which there are no more D3-branes at the bottom of the initial F1-stack. Therefore, all F1strings got attached to these $p$ D5-branes and have been moved away from the main brane configuration along them. This means that the final brane configurations are characterized

\footnotetext{
${ }^{49}$ More precisely, this map between loop operators is found after reabsorbing some signs into imaginary shifts of parameters associated with the flavor Wilson loop (see section 5.4.4).
} 

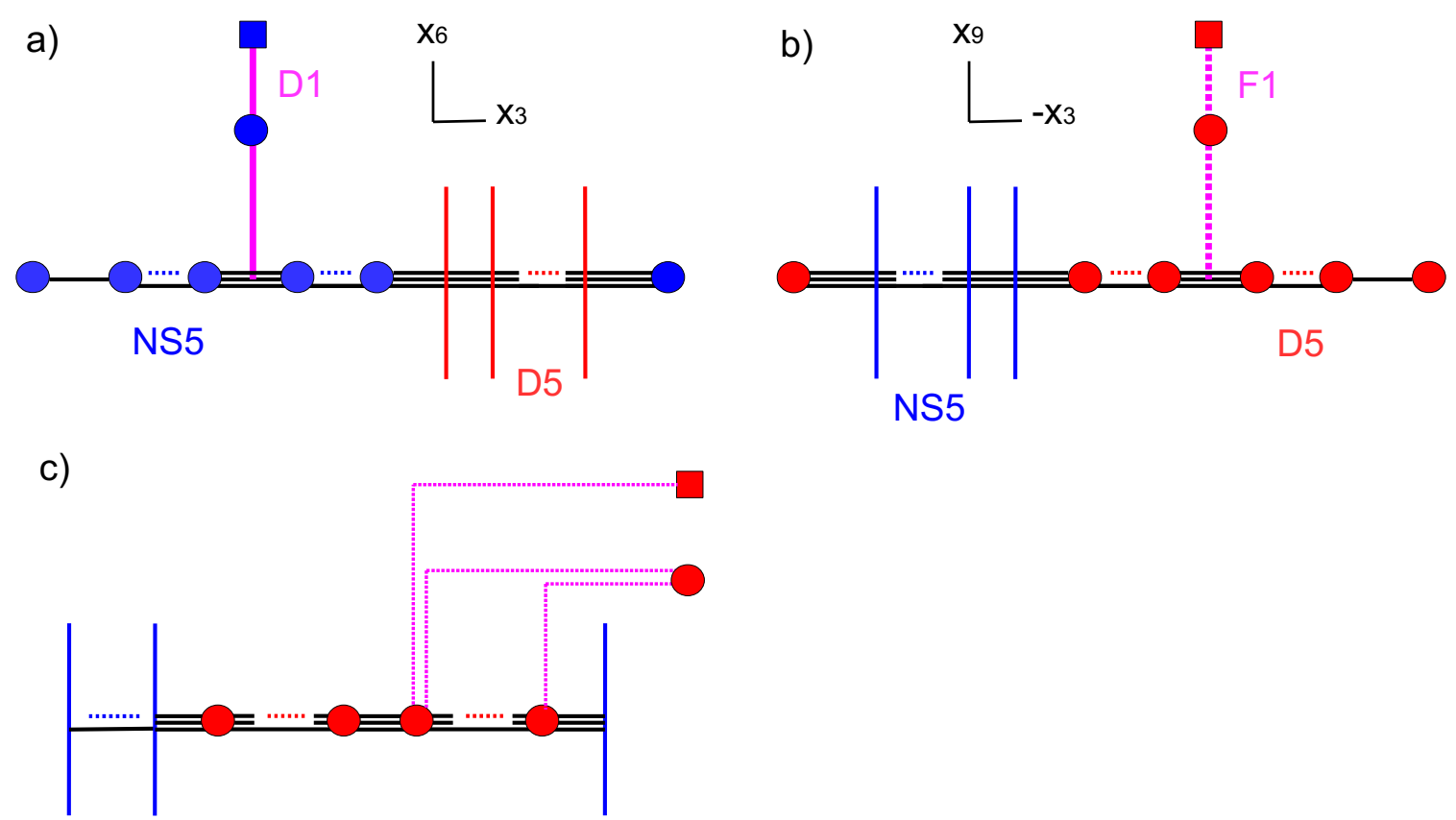

Figure 29. a) Brane configuration inserting a Vortex loop $V_{\mathcal{R}}^{(p)}$. b) S-dual brane configuration. c) Configuration after moving the D5-branes. The F1-strings all end on D5-branes realizing pure flavor Wilson loops.

by having the $k$ F1-strings ending on the $p$ D5-branes on one side and on the $\mathrm{D} 5$ and/or D5' branes on the other side, as in figure 29-c. We interpret this configuration as realizing a non-abelian Wilson loop in the representation $\mathcal{R}$ of the $\mathrm{U}(p)$ flavor symmetry acting on the $p$ hypermultiplets associated to the $p$ D5-branes, which we denote $W_{\mathrm{U}(p), \mathcal{R}}^{\mathrm{f}}$. Therefore, the mirror map is

$$
\left\langle V_{\mathcal{R}}^{(p)}\right\rangle \stackrel{\text { mirror }}{\longleftrightarrow}\left\langle W_{\mathrm{U}(p), \mathcal{R}}^{\mathrm{fl}}\right\rangle \quad 1 \leq p \leq N-2
$$

Likewise, the Vortex loop $V_{N, \mathcal{R}}$ is mirror to a $\mathrm{U}(N-1)$ flavour Wilson loop

$$
\left\langle V_{N, \mathcal{R}}\right\rangle \stackrel{\text { mirror }}{\longleftrightarrow}\left\langle W_{\mathrm{U}(N-1), \mathcal{R}}^{\mathrm{fl}}\right\rangle \text {. }
$$

This concludes our explicit analysis of the mirror map between the loop operators of the self mirror $T[\mathrm{SU}(N)]$ theory.

The inverted map, that is the map from Wilson loops to Vortex loops, can be worked out from the above relations. It relates each Wilson loop to a combination of Vortex loops. Let us provide here some illustrative examples. The mirror maps (4.6) relating the Vortex loops $V_{N-2, \mathcal{S}_{k}}$ and $V_{N-2, \mathcal{A}_{k}}$, labeled by the $k$-(anti)symmetric representation of $\mathrm{U}(N-1)$, to the Wilson loops of the $\mathrm{U}(N-2)$ node of the mirror $T[\mathrm{SU}(N)]$ theory, predicted from the brane picture, are

$$
\begin{aligned}
\left\langle V_{N-2, \mathcal{S}_{k}}\right\rangle \stackrel{\text { mirror }}{\longleftrightarrow}\left\langle\sum_{\hat{k}=0}^{k} W_{N, k-\hat{k}}^{\mathrm{fl}} W_{\mathcal{S}_{\hat{k}}}^{\mathrm{U}(N-2)}\right\rangle, \\
\left\langle V_{N-2, \mathcal{A}_{k}}\right\rangle \stackrel{\text { mirror }}{\longleftrightarrow}\left\langle W_{\mathcal{A}_{k}}^{\mathrm{U}(N-2)}+W_{N, 1}^{\mathrm{fl}} W_{\mathcal{A}_{k-1}}^{\mathrm{U}(N-2)}\right\rangle .
\end{aligned}
$$


Inverting these relations one obtain

$$
\begin{aligned}
& \left\langle W_{\mathcal{S}_{k}}^{\mathrm{U}(N-2)}\right\rangle \stackrel{\text { mirror }}{\longleftrightarrow}\left\langle V_{N-2, \mathcal{S}_{k}}-W_{N, 1}^{\mathrm{fl}} V_{N-2, \mathcal{S}_{k-1}}\right\rangle, \\
& \left\langle W_{\mathcal{A}_{k}}^{\mathrm{U}(N-2)}\right\rangle \stackrel{\text { mirror }}{\longleftrightarrow}\left\langle\sum_{\hat{k}=0}^{k}(-1)^{k-\hat{k}} W_{N, k-\hat{k}}^{\mathrm{fl}} V_{N-2, \mathcal{A}_{\hat{k}}}\right\rangle .
\end{aligned}
$$

It is tempting to try to derive these relation directly from the brane picture. This would necessitate new rules constraining the brane moves which are rather ad hoc. It could be interesting to pursue this.

We have found an explicit map relating Vortex loop operators to gauge and flavor Wilson loop operators. We will check these predictions by exact computations of the expectation value of the loop operators on $S^{3}$ in section 5.4 .

\subsection{Loops and mirror map in circular quivers}

Our prescription to derive the mirror map of loop operators described in section 3 is used here to find the mirror map for loop operators in $3 \mathrm{~d} \mathcal{N}=4$ gauge theories described by circular quivers with equal ranks. The brane realization of this class of quivers is special: after performing S-duality, the branes are already in the canonical brane configuration and no Hanany-Witten moves are required to derive the mirror quiver gauge theory. This immediately implies that a Wilson loop labeled by a representation $\mathcal{R}$ of the gauge group directly maps to a Vortex loop labeled by the same representation $\mathcal{R}$, and not to a sum of Vortex loop operators.

For concreteness, let us consider the circular quiver in figure 30-a, which has two $\mathrm{U}(N)$ gauge group nodes $\mathrm{U}(N)_{a} \times \mathrm{U}(N)_{b}$ and $L \geq 2$ fundamental hypermultiplets of $\mathrm{U}(N)_{b}{ }^{50}$ The mirror dual quiver is easily found by acting with S-duality and is shown in figure 30-b. It has gauge group $\prod_{i=1}^{L} \mathrm{U}(N)_{i}$ and two fundamental hypermultiplets of $\mathrm{U}(N)_{L}$.

Let us first consider a Wilson loop labeled by a representation $\mathcal{R}$ of $\mathrm{U}(N)_{a}$, which we denote by $W_{\mathcal{R}}^{(a)}$. This is realized by the brane configuration in figure 31-a, where F1-strings emanate from extra D5 and/or D5'-branes far away from the main stack and end on the $N$ D3-branes supporting the $\mathrm{U}(N)_{a}$ gauge node. After S-duality the brane configuration is already in its canonical form to read the mirror quiver theory. The F1-strings become D1-branes lying between the two D5-branes and ending on the $N$ D3-branes supporting the the $\mathrm{U}(N)_{L}$ node. This configuration realizes a Vortex loop $\widetilde{V}_{1, \mathcal{R}}^{(L)}$, where the index 1 corresponds to the splitting of the two fundamental hypermultiplets of $\mathrm{U}(N)_{L}$ into $2=1+1$ and $\mathcal{R}$ is now seen as a representation of $\mathrm{U}(N)_{L}$. The operator $\widetilde{V}_{1, \mathcal{R}}^{(L)}$ can be described by a coupled $3 \mathrm{~d} / 1 \mathrm{~d}$ theory which when the D1-branes are moved on top of the nearest NS5brane to their left is summarized by the mixed $3 \mathrm{~d} / 1 \mathrm{~d}$ quiver diagram in $31-\mathrm{a}$. The choice of representation $\mathcal{R}$ is encoded in the $1 \mathrm{~d} \mathcal{N}=4$ quiver gauge theory that is coupled to the $3 \mathrm{~d}$ theory, as explained in section 3.2 .

We now consider a Wilson loop $W_{\mathcal{R}}^{(b)}$ labeled by a representation $\mathcal{R}$ of $\mathrm{U}(N)_{b}$, realized by the brane configuration of figure 31-b. Here we can choose to position the F1-strings so

\footnotetext{
${ }^{50} L \geq 2$ is required for the UV gauge theory to flow to an irreducible SCFT in the IR.
} 
a)
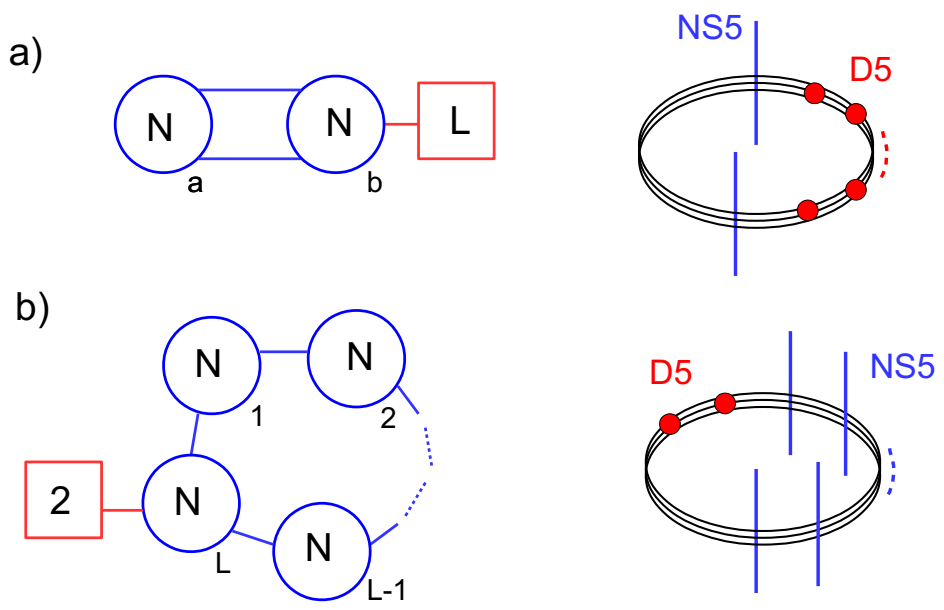

Figure 30. a) $\mathrm{U}(N)_{a} \times \mathrm{U}(N)_{b}$ circular quiver described in the text and its canonical brane configuration. b) Mirror dual circular quiver and brane configuration.

a)

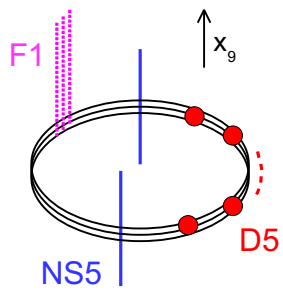

b)

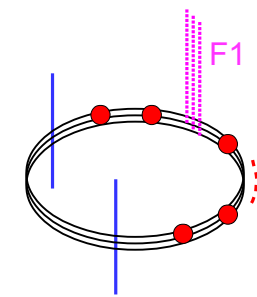

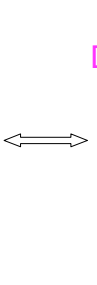
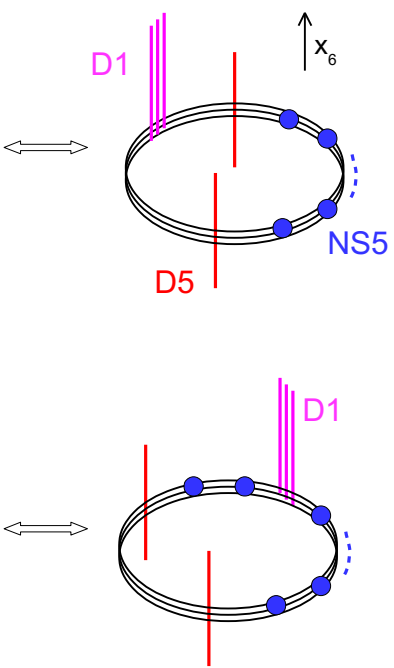

(1d)

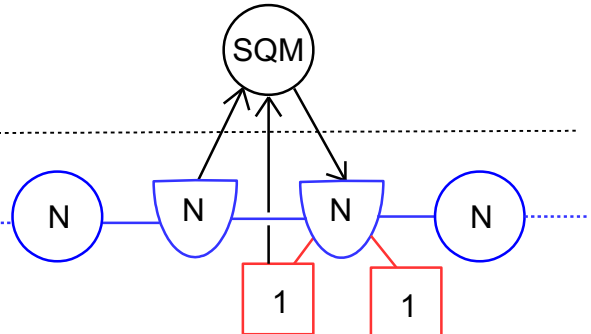

(1d)

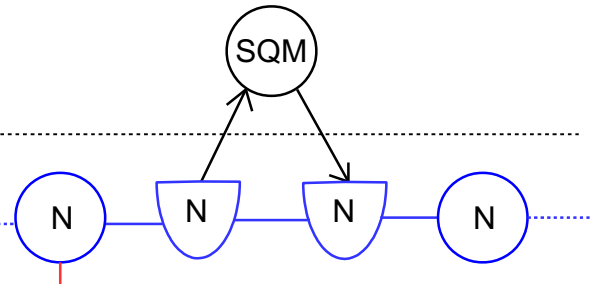

2

Figure 31. a) Brane realization of $W_{\mathcal{R}}^{(a)}$, S-dual brane configuration and 3d/1d defect theory realizing the dual loop $\widetilde{V}_{1, \mathcal{R}}^{(L)}$. The details of the D5/D5' (NS5/NS5') array far from the main stack and the precise $\mathrm{SQM}_{V}$ associated to $\mathcal{R}$ are left unspecified. b) Idem for the Wilson loop $W_{\mathcal{R}}^{(b)}$ and the dual Vortex loop $\widetilde{V}_{\mathcal{R}}^{(m)}$, with $m=2$.

that $m$ D5-branes are to their left and $L-m$ D5-branes are to their right. We note that the choice of $m \in\{0,1, \cdots, L\}$ does not affect the Wilson loop realized by the F1-strings. $S$-duality maps this setup to a brane configuration with D1-branes ending on the $N$ D3branes realizing a Vortex loop $\widetilde{V}_{\mathcal{R}}^{(m)}$ labeled by the same representation $\mathcal{R}$ for the $\mathrm{U}(N)_{m}$ node, for $m=1, \cdots, L-1$. For $m=0$ and $m=L$ S-duality produces the Vortex loop operators $\widetilde{V}_{0, \mathcal{R}}^{(L)}$ and $\widetilde{V}_{2, \mathcal{R}}^{(L)}$ labeled by a representation $\mathcal{R}$ of $\mathrm{U}(N)_{L}$, and where the indices 0 and 2 refer to the remaining hypermultiplet splittings $2=0+2=2+0$. The operator 

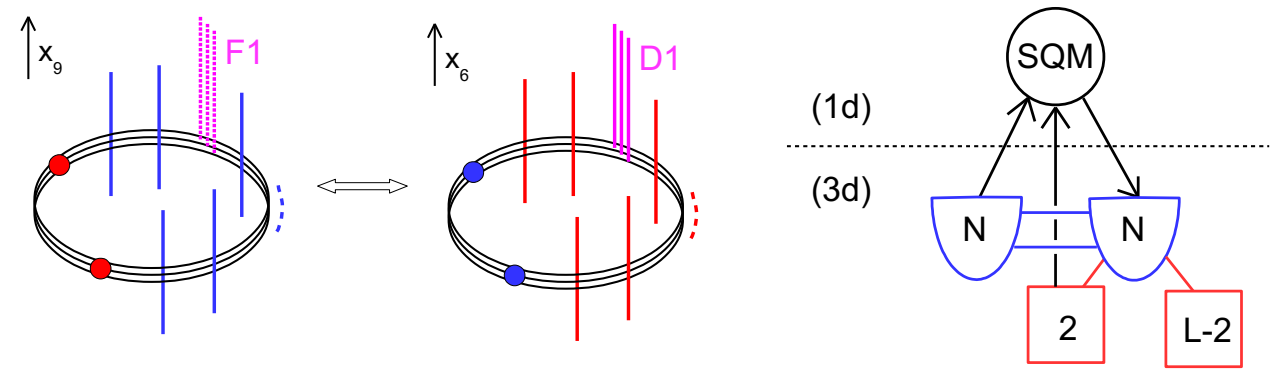

Figure 32. a) Brane realization of $\widetilde{W}_{\mathcal{R}}^{(m)}$, S-dual brane configuration and 3d/1d defect theory realizing the dual loop $V_{m, \mathcal{R}}^{(b)}$, for $m=2$.

$\widetilde{V}_{\mathcal{R}}^{(m)}$ can be described by a coupled 3d/1d theory, which when obtained by moving the D1-branes on top of the nearest NS5-brane to their left, is summarized by the mixed $3 \mathrm{~d} / 1 \mathrm{~d}$ quiver diagram in figure 31-b (for $m=2$ ). We leave as an exercise for the interested reader to identify the the $3 \mathrm{~d} / 1 \mathrm{~d}$ quiver theories describing $\widetilde{V}_{0, \mathcal{R}}^{(L)}$ and $\widetilde{V}_{2, \mathcal{R}}^{(L)}$.

When giving a brane description of the Wilson loop $W_{\mathcal{R}}^{(b)}$ we had a choice of where to insert the F1-strings relative to the D5-branes, a choice that does not affect the choice of Wilson loop. This arbitrariness has an interesting counterpart in the mirror dual theory. Different choices of $m$ result in different $3 \mathrm{~d} / 1 \mathrm{~d}$ quiver gauge theories that describe the same mirror Vortex loop operator in the IR. These are dual descriptions of the same IR operator and are akin to the hopping duality introduced in [21] (see also [8]) in the context of surface operators in $4 \mathrm{~d} \mathcal{N}=2$ gauge theories.

In summary, we find the following mirror maps

$$
\begin{aligned}
& \left\langle W_{\mathcal{R}}^{(a)}\right\rangle \stackrel{\text { mirror }}{\longleftrightarrow}\left\langle\widetilde{V}_{1, \mathcal{R}}^{(L)}\right\rangle \\
& \left\langle W_{\mathcal{R}}^{(b)}\right\rangle \stackrel{\text { mirror }}{\longleftrightarrow}\left\langle\widetilde{V}_{\mathcal{R}}^{(m)}\right\rangle=\left\langle\widetilde{V}_{0, \mathcal{R}}^{(L)}\right\rangle=\left\langle\widetilde{V}_{2, \mathcal{R}}^{(L)}\right\rangle, m=1, \cdots, L-1 .
\end{aligned}
$$

The mirror map between Vortex loops of the initial quiver in figure 31-a and Wilson loops of the mirror quiver in 31-b can be found in the same way. The two-node quiver has Vortex loops $V_{\mathcal{R}}^{(a)}$ in the $\mathrm{U}(N)_{a}$ node and $V_{m, \mathcal{R}}^{(b)}$ in the $\mathrm{U}(N)_{b}$ node, with $m$ denoting the splitting of hypermultiplets $L=m+(L-m)$ for $m=0, \cdots, L$. The $L$-node quiver has Wilson loops $\widetilde{W}_{\mathcal{R}}^{(m)}$ in the $\mathrm{U}(N)_{m}$ node, with $m=1, \cdots, L$. The brane realizations of these loop operators are easily mapped under S-duality, as in the example of figure 32. The precise mirror map is

$$
\begin{aligned}
\left\langle V_{\mathcal{R}}^{(a)}\right\rangle=\left\langle V_{0, \mathcal{R}}^{(b)}\right\rangle= & \left\langle V_{L, \mathcal{R}}^{(b)}\right\rangle \stackrel{\text { mirror }}{\longleftrightarrow}\left\langle\widetilde{W}_{\mathcal{R}}^{(L)}\right\rangle \\
& \left\langle V_{m, \mathcal{R}}^{(b)}\right\rangle \stackrel{\text { mirror }}{\longleftrightarrow}\left\langle\widetilde{W}_{\mathcal{R}}^{(m)}\right\rangle, m=1, \cdots, L-1 .
\end{aligned}
$$

We find once again that some Vortex loops have inequivalent descriptions, predicting hopping dualities for the associated $3 \mathrm{~d} / 1 \mathrm{~d}$ theories.

Other circular quivers with nodes of equal rank and arbitrary number of fundamental hypermultiplets in each node can be treated similarly, leading to explicit maps between loop operators labeled by the same representation $\mathcal{R}$. For circular quivers with varying 
a)
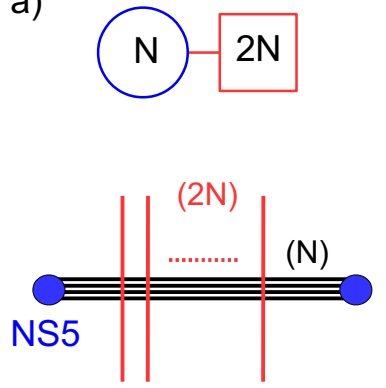

D5 b)

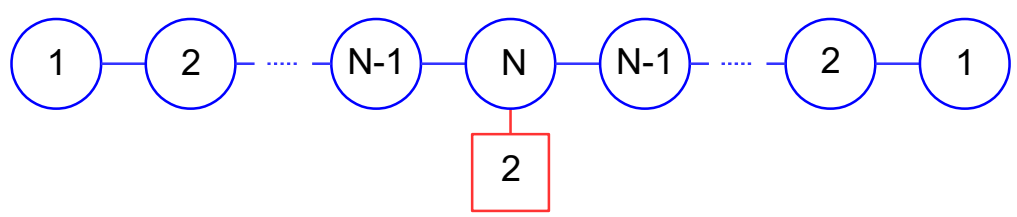

Figure 33. a) Quiver and brane realization of the $\mathrm{U}(N)$ theory with $2 N$ fundamental hypermultiplets. b) Mirror theory and brane configuration.

ranks, one has to rely on the more elaborate analysis presented in section 3.2 , which still results in explicit mirror maps between loop operators very similar to those in section 4.3.

\subsection{Loops in SQCD with $2 N$ quarks and its mirror}

For our final example we consider $3 \mathrm{~d} \mathcal{N}=4 \mathrm{SQCD}$, with $\mathrm{U}(N)$ gauge group and $2 N$ fundamental hypermultiplets, which is the smallest number of fundamental hypermultiplets $\left(N_{f} \geq 2 N_{c}\right)$ required for SQCD to flow in the IR to an irreducible SCFT. The quiver diagram and brane realization of this theory is given in figure 33-a.

This theory has Wilson loops $W_{\mathcal{R}}$, labeled by a representation $\mathcal{R}$ of $\mathrm{U}(N)$ and Vortex loops $V_{M, \mathcal{R}}$, labeled by a representation $\mathcal{R}$ of $\mathrm{U}(N)$ and a splitting $2 N=M+(2 N-M)$ of the fundamental hypermultiplets. The brane realization and corresponding $3 \mathrm{~d} / 1 \mathrm{~d}$ quiver representation of $V_{M, \mathcal{R}}$ are shown in figure 34-a. As before, the choice of representation $\mathcal{R}$ is encoded in the $1 \mathrm{~d} \mathcal{N}=4$ quiver gauge theory that is coupled to the $3 \mathrm{~d}$ theory, as explained in section 3.2.

The mirror theory is shown is figure 33-b. It is a quiver with gauge group $\prod_{n=1}^{N-1} \mathrm{U}(n)_{a} \times$ $\mathrm{U}(N) \times \prod_{n=1}^{N-1} \mathrm{U}(n)_{b}$ with two fundamental hypermultiplets in the $\mathrm{U}(N)$ node. The indices $a$ and $b$ distinguish the nodes on the left and on the right of the central node of the quiver. This theory has Wilson loops $\widetilde{W}_{\mathcal{R}}^{(a, n)}$ in the $\mathrm{U}(n)_{a}$ nodes, $\widetilde{W}_{\mathcal{R}}^{(N)}$ in the $\mathrm{U}(N)$ node and $\widetilde{W}_{\mathcal{R}}^{(b, n)}$ in the $\mathrm{U}(n)_{b}$ nodes. We denote the Vortex loops of this mirror theory by $\widetilde{V}_{\mathcal{R}}^{(a, n)}$ and $\widetilde{V}_{\mathcal{R}}^{(b, n)}$ for the loops coupled to the $\mathrm{U}(N)_{a}$ and $\mathrm{U}(N)_{b}$ nodes and $\widetilde{V}_{0, \mathcal{R}}^{(N)}, \widetilde{V}_{1, \mathcal{R}}^{(N)}, \widetilde{V}_{2, \mathcal{R}}^{(N)}$ the Vortex loops of the central $\mathrm{U}(N)$ node, labeled by a splitting of the two fundamental hypermultiplets $(2=0+2=1+1=0+2$ respectively). We show the brane realization and associated $3 \mathrm{~d} / 1 \mathrm{~d}$ quiver for the Vortex loop $\widetilde{V}_{1, \mathcal{R}}^{(N)}$ in figure 34 -b.

We now derive the mirror maps using the brane picture. Consider first the Wilson loop $W_{\mathcal{R}}$ of the SQCD theory. It is realized with F1-strings ending on the $N$ D3-branes supporting the $\mathrm{U}(N)$ gauge group, placed among the $2 N$ D5-branes at our convenience. We choose to place the strings with $N$ D5-branes on each side. After S-duality and D5brane rearrangements, we end up with D1-branes ending on the $N$ D3-branes supporting the central $\mathrm{U}(N)$ gauge node of the mirror theory, placed between the two D5-branes. This is summarized in figure 35 . This configuration realizes the vortex loop $\widetilde{V}_{1, \mathcal{R}}$, which is 
a)

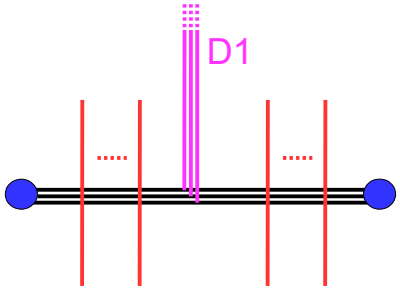

(M)

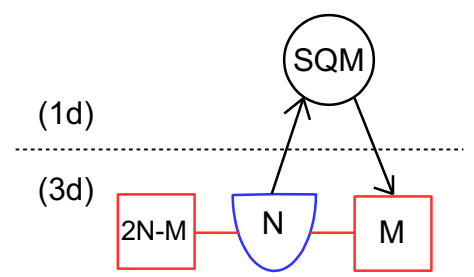

$(2 \mathrm{~N}-\mathrm{M})$
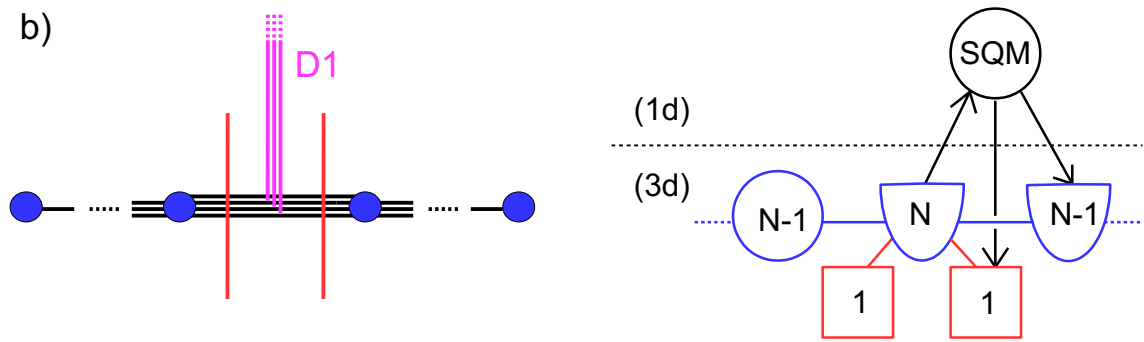

Figure 34. a) Brane realization of a Vortex loop $V_{M, \mathcal{R}}$ in the $\mathrm{U}(N)$ theory and associated 'right' $3 \mathrm{~d} / 1 \mathrm{~d}$ quiver. b) Idem for a Vortex loop $\widetilde{V}_{1, \mathcal{R}}^{(N)}$ of the mirror theory.
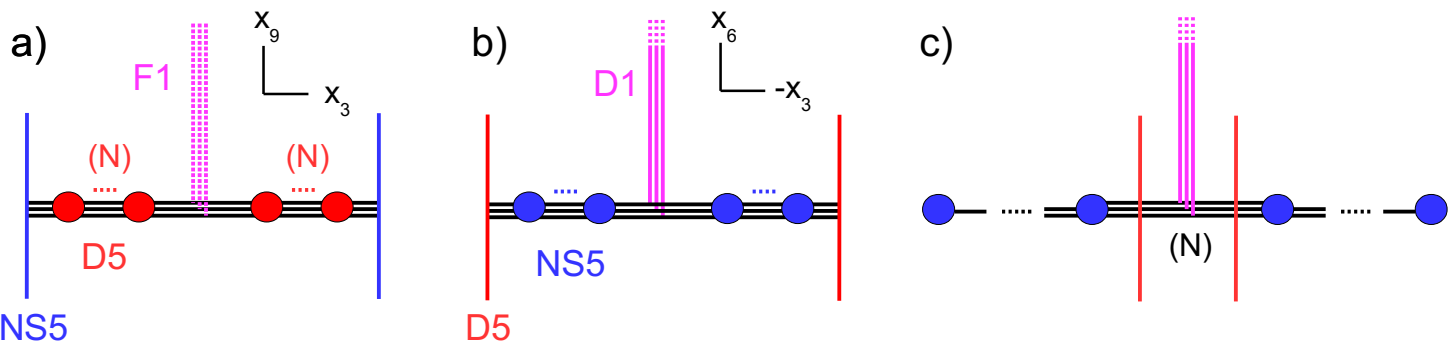

Figure 35. a) Brane realization of a $\mathrm{U}(N)$ Wilson loop $W_{\mathcal{R}}$. b) S-dual brane configuration with D1-branes (and $x^{3}$ reflection). c) After D5-brane moves the D1-branes end on the $N$ D3brane supporting the central $\mathrm{U}(N)$ node of the mirror theory and are placed between the two D5s, realizing the Vortex loop $\widetilde{V}_{1, \mathcal{R}}^{(N)}$.

labeled by the same representation $\mathcal{R}$ of $\mathrm{U}(N)$ as the initial Wilson loop. The mirror map is then simply

$$
\left\langle W_{\mathcal{R}}\right\rangle \stackrel{\text { mirror }}{\longleftrightarrow}\left\langle\widetilde{V}_{1, \mathcal{R}}^{(N)}\right\rangle .
$$

Consider now a Vortex loop $V_{M, \mathcal{R}}$ of the SQCD theory and assume $M \leq N$. The loop is realized with D1-branes ending on the $N$ D3-branes, with $M$ D5-branes on its right and $2 N-M$ D5-branes on its left. After S-duality (and $x^{3}$ reflection), we end up with the configuration in figure 36-b. The D5-brane on the left of the picture has to be moved across the F1-strings. This is the same situation as encountered when studying $T[\mathrm{SU}(N)]$ Vortex loops. When the D5 crosses the F1-strings the number of D3-branes at the bottom of the strings diminishes and part of the strings get attached to the D5-brane. The final brane configurations have F1-strings ending on the $M$ D3-branes supporting the $\mathrm{U}(M)_{a}$ gauge node and F1-strings ending on the left D5-brane, as in figure 36-c. They 


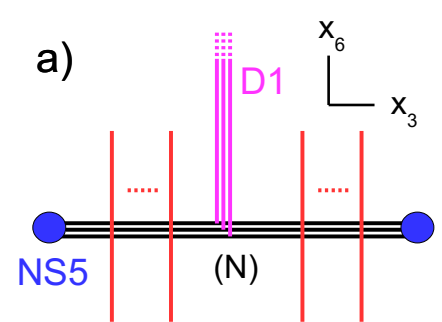

(2N-M)

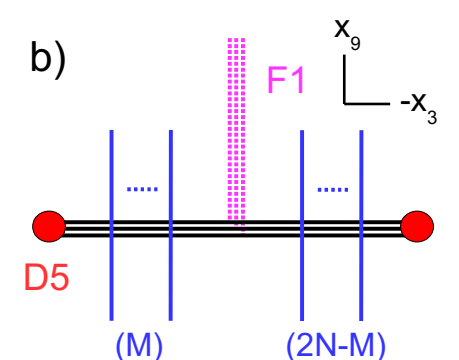

(M)

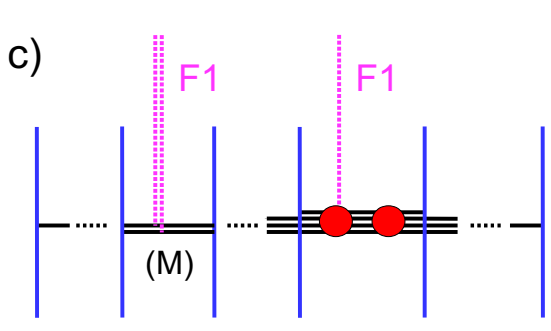

Figure 36. a) Brane realization of the Vortex loop $V_{M, \mathcal{R}}$ with $M \leq N$. b) S-dual brane configuration with F1-strings (and $x^{3} \rightarrow-x^{3}$ ). c) After D5-brane moves, part of the strings end on the left D5 (flavor Wilson loop) and the other strings end on the $M$ D3-branes supporting the $\mathrm{U}(M)_{a}$ node (gauge Wilson loop).

realize Wilson loops $\widetilde{W}_{\widehat{\mathcal{R}}}^{(a, M)}$ in the $\mathrm{U}(M)_{a}$ node, together with flavor Wilson loops $\widetilde{W}_{1, \mathbf{q}}^{\mathrm{f}}$ of charge $\mathbf{q}$ under the $\mathrm{U}(1)$ flavour symmetry rotating the hypermultiplet associated to the left D5-brane, where the representation $(\mathbf{q}, \widehat{\mathcal{R}})$ appears in the decomposition of the representation $\mathcal{R}$ under the subgroup $\mathrm{U}(1) \times \mathrm{U}(M)$ of $\mathrm{U}(N)$, where $\mathrm{U}(M)$ is embedded as $\mathrm{U}(M) \times \mathrm{U}(N-M) \subset \mathrm{U}(N)$ and $\mathrm{U}(1)$ is embedded diagonally in $\mathrm{U}(N-M)$.

Following the arguments that we presented for the $T[\mathrm{SU}(N)]$ loops in 4.3 , we arrive at the explicit mirror map

$$
\left\langle V_{M, \mathcal{R}}\right\rangle \stackrel{\text { mirror }}{\longleftrightarrow}\left\langle\sum_{s \in \Delta_{M}} \widetilde{W}_{1, \mathbf{q}_{s}}^{\mathrm{fl}} \widetilde{W}_{\widehat{\mathcal{R}}_{s}}^{(a, M)}\right\rangle, \quad 0 \leq M \leq N,
$$

where $\Delta_{M}$ denotes the set of representations $(\mathbf{q}, \widehat{\mathcal{R}})$ in the decomposition of $\mathcal{R}$ under $\mathrm{U}(N) \rightarrow \mathrm{U}(1) \times \mathrm{U}(M)$, counted with degeneracies. This is completely analogous to the mirror maps found for the $T[\mathrm{SU}(N)]$ theory.

The map relating the Vortex loops $V_{M, \mathcal{R}}$ with $M \geq N$ to combinations of Wilson loops $\widetilde{W}_{2, \mathbf{q}}^{\mathrm{fl}} \widetilde{W}_{\widehat{\mathcal{R}}}^{b, M}$ is found similarly, with $\widetilde{W}_{2, \mathbf{q}}^{\mathrm{fl}}$ denoting a flavor Wilson loop of charge $\mathbf{q}$ under the $\mathrm{U}(1)$ flavour symmetry rotating the hypermultiplet associated to the right D5-brane this time. Note that the 'central' Vortex loop $V_{N, \mathcal{R}}$ is directly mapped to the Wilson loop $\widetilde{W}_{\mathcal{R}}^{(N)}$ of the central node of the mirror theory:

$$
\left\langle V_{N, \mathcal{R}}\right\rangle \stackrel{\text { mirror }}{\longleftrightarrow}\left\langle\widetilde{W}_{\mathcal{R}}^{(N)}\right\rangle
$$

The only loops we did not discuss thus far are the Vortex loops $\widetilde{V}_{\mathcal{R}}^{(a, n)}, \widetilde{V}_{\mathcal{R}}^{(b, n)}$ of the $\mathrm{U}(n)_{a}$ and $\mathrm{U}(n)_{b}$ nodes of the mirror theory, and the $\widetilde{V}_{0, \mathcal{R}}^{(N)}, \widetilde{V}_{2, \mathcal{R}}^{(N)}$ Vortex loops of the central $\mathrm{U}(N)$ node, which can be naturally renamed as $\widetilde{V}_{\mathcal{R}}^{(a, N)}$ and $\widetilde{V}_{\mathcal{R}}^{(b, N)}$ respectively. The analysis of the brane realization of these loops is the same as for the Vortex loops $V_{\mathcal{R}}^{(p)}$ of the $T[\mathrm{SU}(N)]$ theory (see section 4.3) and lead to the conclusion that these loops are mapped to flavor Wilson loops. In particular

$$
\begin{aligned}
& \left\langle\widetilde{V}_{\mathcal{R}}^{(a, n)}\right\rangle=\left\langle W_{\mathrm{U}(n)_{F}, \mathcal{R}}^{\mathrm{f}}\right\rangle, \quad n=1, \cdots, N, \\
& \left\langle\widetilde{V}_{\mathcal{R}}^{(b, n)}\right\rangle=\left\langle W_{\mathrm{U}(n)_{F^{\prime}}, \mathcal{R}}^{\mathrm{f}}\right\rangle, \quad n=1, \cdots, N,
\end{aligned}
$$


where $\mathrm{U}(n)_{F}$ is the flavor group acting on the $n$ hypermultiplets associated to the $n$ leftmost D5-branes, $\mathrm{U}(n)_{F^{\prime}}$ is the flavor group acting on the $n$ hypermultiplets associated to the $n$ rightmost D5-branes and $W_{\mathrm{U}(n), \mathcal{R}}^{\mathrm{f}}$ denotes a flavor Wilson loop in the representation $\mathcal{R}$ of $\mathrm{U}(n)$.

This completes the derivation of the mirror map between the loops of the $\mathrm{U}(N)$ theory with $2 N$ fundamental hypermultiplets and its mirror dual. The analysis generalizes easily to the $\mathrm{U}(N)$ theory with $N_{f} \geq 2 N$ fundamental hypermultiplets.

\section{Loop operators in $3 \mathrm{~d} \mathcal{N}=4$ theories on $S^{3}$}

In this section we provide explicit quantitative evidence for our proposed action of mirror symmetry on loop operators by computing the exact expectation value of supersymmetric loop operators on $S^{3}$ in $3 \mathrm{~d} \mathcal{N}=4$ gauge theories. We confirm our proposal for the action of mirror symmetry on loop operators by showing that the expectation value of mirror loop operators match perfectly and indeed give alternative UV descriptions of superconformal line defects in the IR SCFT.

Our first task is to define these observables in $\mathcal{N}=4$ theories on $S^{3}$, where exact computations can be performed by supersymmetric localization. Recall that in $R^{3}$ the UV Lagrangian definition of a $3 \mathrm{~d} \mathcal{N}=4$ SCFT is invariant under those supercharges in $\operatorname{OSp}(4 \mid 4)$ that close into the isometries of flat space, which generate the $3 \mathrm{~d} \mathcal{N}=4$ superPoincaré algebra. ${ }^{51}$ While an $\mathcal{N}=4$ SCFT can be placed canonically on the round $S^{3}$ by stereographic projection, its UV Lagrangian description likewise breaks $\operatorname{OSp}(4 \mid 4)$ to a subalgebra, which we now identify. On $S^{3}$, the supersymmetries of a generic UV (massive) $3 \mathrm{~d} \mathcal{N}=4$ theory are generated by the supercharges in $\operatorname{OSp}(4 \mid 4)$ that close into the $\mathrm{SU}(2)_{l} \times$ $\mathrm{SU}(2)_{r}$ isometries of $S^{3}$ and project out the remaining $\mathrm{SO}(4,1)$ conformal generators on $S^{3}$. Since we allow arbitrary massive deformations, and mass and FI parameters transform in the $(\mathbf{3}, \mathbf{1})$ and $(\mathbf{1}, \mathbf{3})$ representations of $\mathrm{SU}(2)_{C} \times \mathrm{SU}(2)_{H}$, the R-symmetry in $\mathrm{OSp}(4 \mid 4)$ is broken down to its Cartan subalgebra on $S^{3}$. Under the $\mathrm{SU}(2)_{l} \times \mathrm{SU}(2)_{r} \times \mathrm{U}(1)_{C} \times \mathrm{U}(1)_{H}$ embedding in $\mathrm{SO}(4,1) \times \mathrm{SU}(2)_{C} \times \mathrm{SU}(2)_{H}$, the supercharges generating $\mathrm{OSp}(4 \mid 4)$, which transform in the $(\mathbf{4}, \mathbf{2}, \mathbf{2})$, decompose as $(\mathbf{2}, \mathbf{1})_{++} \oplus(\mathbf{2}, \mathbf{1})_{+-} \oplus(\mathbf{2}, \mathbf{1})_{-+} \oplus(\mathbf{2}, \mathbf{1})_{--} \oplus(\mathbf{1}, \mathbf{2})_{++} \oplus$ $(\mathbf{1}, \mathbf{2})_{--} \oplus(\mathbf{1}, \mathbf{2})_{-+} \oplus(\mathbf{1}, \mathbf{2})_{--}$. The supersymmetries preserved by a UV $3 \mathrm{~d} \mathcal{N}=4$ theory on $S^{3}$ are $(\mathbf{2}, \mathbf{1})_{++} \oplus(\mathbf{2}, \mathbf{1})_{--} \oplus(\mathbf{1}, \mathbf{2})_{+-} \oplus(\mathbf{1}, \mathbf{2})_{-+}$. These supercharges generate the following supergroup ${ }^{52}$

$$
\mathrm{SU}(2 \mid 1)_{l} \times \mathrm{SU}(2 \mid 1)_{r}
$$

The $\mathrm{U}(1)_{l}$ generator in $\mathrm{SU}(2 \mid 1)_{l}$ is $R_{C}+R_{H}$ while the $\mathrm{U}(1)_{r}$ generator in $\mathrm{SU}(2 \mid 1)_{l}$ is $R_{H}-R_{C}$, where $R_{C}$ and $R_{H}$ are the Cartan generators of $\mathrm{SU}(2)_{C}$ and $\mathrm{SU}(2)_{H}$ respectively. The isometries of $S^{3}$ are $\mathrm{SU}(2)_{l} \times \mathrm{SU}(2)_{r}$. Explicitly, the anticommutators of the $\mathrm{SU}(2 \mid 1)_{l} \times$

\footnotetext{
${ }^{51}$ Super-Euclidean in $R^{3}$.

${ }^{52}$ This supersymmetry is the fixed locus of an involution in $\operatorname{OSp}(4 \mid 4)$.
} 
$\mathrm{SU}(2 \mid 1)_{r}$ supercharges on an $S^{3}$ of radius $L$ are

$$
\begin{aligned}
\left\{Q_{l \alpha}, \bar{Q}_{l \beta}\right\} & =\frac{2}{L}\left[\left(\gamma^{a} C^{-1}\right)_{\alpha \beta} J_{a}^{l}+\frac{1}{2} \epsilon_{\alpha \beta}\left(R_{C}+R_{H}\right)\right] \\
\left\{Q_{r \alpha}, \bar{Q}_{r \beta}\right\} & =\frac{2}{L}\left[\left(\gamma^{a} C^{-1}\right)_{\alpha \beta} J_{a}^{r}+\frac{1}{2} \epsilon_{\alpha \beta}\left(R_{C}-R_{H}\right)\right],
\end{aligned}
$$

where $J_{a}^{l}$ and $J_{a}^{r}$ generate $\mathrm{SU}(2)_{l}$ and $\mathrm{SU}(2)_{r}$ respectively.

In the flat space $L \rightarrow \infty$ limit, the $\mathrm{SU}(2 \mid 1)_{l} \times \mathrm{SU}(2 \mid 1)_{r}$ symmetry on the $S^{3}$ contracts to the $3 \mathrm{~d} \mathcal{N}=4$ super-Poincaré symmetry. Indeed, writing $J_{a}^{l}=\frac{1}{2}\left(J_{a}+L P_{a}\right)$ and $J_{a}^{r}=$ $\frac{1}{2}\left(J_{a}-L P_{a}\right)$, and taking the flat space limit we get

$$
\begin{aligned}
\left\{Q_{l \alpha}^{\infty}, \bar{Q}_{l \beta}^{\infty}\right\} & =\left(\gamma^{a} C^{-1}\right)_{\alpha \beta} P_{a} \\
\left\{Q_{r \alpha}^{\infty}, \bar{Q}_{r \beta}^{\infty}\right\} & =-\left(\gamma^{a} C^{-1}\right)_{\alpha \beta} P_{a},
\end{aligned}
$$

where $P_{a}$ and $J_{a}$ are the translation and rotation generators of $R^{3}$. Comparing with the $3 \mathrm{~d} \mathcal{N}=4$ super-Poincaré supercharges $Q_{\alpha A A^{\prime}}$ in (2.1) we find the identification: $Q_{l \alpha}^{\infty}=$ $Q_{\alpha 22}, \bar{Q}_{l \alpha}^{\infty}=Q_{\alpha 11}$, $Q_{r \alpha}^{\infty}=Q_{\alpha 21}$ and $\bar{Q}_{r \alpha}^{\infty}=Q_{\alpha 12}$.

The $\mathrm{SU}(2 \mid 1)_{l}$ supersymmetry transformations are generated by Killing spinors $\epsilon_{l}$ and $\bar{\epsilon}_{l}$ on $S^{3}$ obeying $[17]^{53}$

$$
\nabla_{\mu} \epsilon_{l}=\frac{i}{2 L} \gamma_{\mu} \epsilon_{l} \quad \nabla_{\mu} \bar{\epsilon}_{l}=\frac{i}{2 L} \gamma_{\mu} \bar{\epsilon}_{l}
$$

while $\mathrm{SU}(2 \mid 1)_{r}$ transformations are generated by Killing spinors $\epsilon_{r}$ and $\bar{\epsilon}_{r}$ on $S^{3}$ which satisfy [39]

$$
\nabla_{\mu} \epsilon_{r}=-\frac{i}{2 L} \gamma_{\mu} \epsilon_{r} \quad \nabla_{\mu} \bar{\epsilon}_{r}=-\frac{i}{2 L} \gamma_{\mu} \bar{\epsilon}_{r}
$$

From the viewpoint of off-shell supersymmetric supergravity backgrounds [40], these Killing spinor equations arise from $3 \mathrm{~d} \mathcal{N}=4$ supergravity in the presence of a background auxiliary field. ${ }^{54}$ The superisometry of this supergravity background is $\mathrm{SU}(2 \mid 1)_{l} \times \mathrm{SU}(2 \mid 1)_{r}$.

We take the left-invariant frame $e^{a}=L \mu^{a}, a=1,2,3$, where the left-invariant oneforms of SU(2) are defined by $g^{-1} d g=i \mu^{a} \tau_{a}$ with $g=\left(\begin{array}{cc}z_{1} & z_{2} \\ -\bar{z}_{2} & \bar{z}_{1}\end{array}\right)$ so that $\operatorname{det} g=1$. In this frame $\epsilon_{l}$ and $\bar{\epsilon}_{l}$ are constant while $\epsilon_{r}$ and $\bar{\epsilon}_{r}$ are given by $\epsilon_{r}=g^{-1} \epsilon_{0}, \bar{\epsilon}_{r}=g^{-1} \widetilde{\epsilon}_{0}$, with $\epsilon_{0}, \widetilde{\epsilon}_{0}$ constant. ${ }^{55}$

The explicit $3 \mathrm{~d} \mathcal{N}=4$ supersymmetric Lagrangians on $S^{3}$ can be written down using the formulae for $3 \mathrm{~d} \mathcal{N}=2$ gauge theories on $S^{3}$ in [17, 39, 42-44], which are invariant under $\mathrm{SU}(2 \mid 1)_{l} \times \mathrm{SU}(2)_{r}$. We first decompose the $\mathcal{N}=4$ vector multiplet and hypermultiplet into $\mathcal{N}=2$ vector and chiral multiplets. By assigning $\mathrm{U}(1)_{l}$ charge 1 and $1 / 2$ to the chiral

\footnotetext{
${ }^{53}$ All these Killing spinors are the subset of conformal Killing spinors on $S^{3}$, which obey $\nabla_{\mu} \epsilon_{A A^{\prime}}=$ $\frac{1}{3} \gamma_{\mu} \not \epsilon_{A A^{\prime}}$, that project out the conformal generators on $S^{3}$.

${ }^{54}$ The background fields in supergravity and conformal compensators are very similar to those required to put $4 \mathrm{~d} \mathcal{N}=2$ theories on $S^{4}$, see [41].

${ }^{55}$ We note that all Killing spinors generating $\mathrm{SU}(2 \mid 1)_{l} \times \mathrm{SU}(2 \mid 1)_{r}$ are periodic along the Hopf circle.
} 
multiplets inside an $\mathcal{N}=4$ vector multiplet and hypermultiplet respectively, the supersymmetry of such an $\mathcal{N}=2$ theory on $S^{3}$ is enhanced with extra four supercharges [39], which generate the remaining $\mathrm{SU}(2 \mid 1)_{r}$. Just as in flat space, $3 \mathrm{~d} \mathcal{N}=4$ theories on $S^{3}$ admit canonical relevant deformations associated to the $G_{C} \times G_{H}$ symmetries of the IR SCFT. These are introduced by turning on $\mathrm{SU}(2 \mid 1)_{l} \times \mathrm{SU}(2 \mid 1)_{r}$ invariant background vector and twisted vector multiplets on $S^{3}$ for $G_{H}$ and $G_{C}$ respectively. Supersymmetric backgrounds for $3 \mathrm{~d} \mathcal{N}=4$ vector and twisted vector multiples on $S^{3}$ allow a single scalar in the multiplet to be turned on, instead of the triplet of parameters in flat space. Therefore on $S^{3}$ there is a single mass parameter and a single FI for each Cartan generator in $G_{H}$ and $G_{C}$ respectively.

Following a similar analysis to the one in flat space in section 2, there are two inequivalent classes of half-supersymmetric line operators that can be defined in a UV description of a $3 \mathrm{~d} \mathcal{N}=4$ SCFT on $S^{3}$. They are characterised by two different embeddings of $\mathrm{SU}(1 \mid 1)_{l} \times \mathrm{SU}(1 \mid 1)_{r}$ inside $\mathrm{SU}(2 \mid 1)_{l} \times \mathrm{SU}(2 \mid 1)_{r}$. One class corresponds to supersymmetric Wilson loop operators and the other to Vortex loop operators on $S^{3}$.

\subsection{Wilson loops on $S^{3}$}

Consider a Wilson loop operator wrapping a curve $\gamma \in S^{3}$

$$
\operatorname{Tr}_{R} P \exp \oint_{\gamma}\left(i A_{\mu} \dot{x}^{\mu}-|\dot{x}| \sigma\right) d \tau
$$

in a $3 \mathrm{~d} \mathcal{N}=4$ gauge theory, where $\sigma$ is the scalar field in the $\mathcal{N}=2$ vector multiplet inside the $\mathcal{N}=4$ vector multiplet. This operator preserves the $\mathrm{SU}(2 \mid 1)_{l} \times \mathrm{SU}(2 \mid 1)_{r}$ Killing spinors that are solutions to the following equations on $S^{3}$ (see appendix A) ${ }^{56}$

$$
\begin{aligned}
\left(\gamma_{\mu} \dot{x}^{\mu}-|\dot{x}|\right) \epsilon_{l}=0 & \left(\gamma_{\mu} \dot{x}^{\mu}+|\dot{x}|\right) \bar{\epsilon}_{l}=0 \\
\left(\gamma_{\mu} \dot{x}^{\mu}-|\dot{x}|\right) \epsilon_{r}=0 & \left(\gamma_{\mu} \dot{x}^{\mu}+|\dot{x}|\right) \bar{\epsilon}_{r}=0 .
\end{aligned}
$$

By a choice of parametrization of the loop $\gamma \in S^{3}$ we can always make $|\dot{x}|=1$. Since in the $\mathrm{SU}(2)_{l}$ left-invariant frame the Killing spinors $\epsilon_{l}$ and $\bar{\epsilon}_{l}$ are constant, imposing that the Wilson loop preserves half of the supercharges in $\mathrm{SU}(2 \mid 1)_{l}$ requires that

$$
\dot{x}^{\mu}=n^{a} e_{a}^{\mu},
$$

where $n^{a}$ is a constant unit three-vector $n^{a} n^{a}=1$. A Wilson loop wrapping the curve $\dot{x}^{\mu}=n^{a} e_{a}^{\mu}$ on $S^{3}$ preserves the following half of the $\mathrm{SU}(2 \mid 1)_{l}$ supersymmetries

$$
\gamma_{a} n^{a} \epsilon_{l}=\epsilon_{l} \quad \gamma_{a} n^{a} \bar{\epsilon}_{l}=-\bar{\epsilon}_{l}
$$

The integral curves of the vector field $\dot{x}^{m}=n^{a} e_{a}^{m}$ are great circles on $S^{3}$. The choice of unit vector $n^{a}$ determines a Hopf fibration. Therefore, there is an $S^{2}=\mathrm{SU}(2) / \mathrm{U}(1)$ worth of choices of Hopf fibrations. Given a choice of Hopf fibration, there is another $S^{2}=\mathrm{SU}(2) / \mathrm{U}(1)$ worth of Hopf circles, labeled by the point on $S^{2}$ where the Hopf fiber

\footnotetext{
${ }^{56}$ These equations hold at the position of the loop.
} 
sits. This realizes the space of maximal circles on $S^{3}$ as $\mathrm{SO}(4) / \mathrm{U}(1) \times \mathrm{U}(1)=S^{2} \times S^{2}$. Note also that all circles in a choice of Hopf fibration preserve the same supersymmetry in $\mathrm{SU}(2 \mid 1)_{l}$.

Without loss of generality we take $n^{a}=(0,0,1)$. The Hopf circle preserves $J_{3}^{l}$ generating $\mathrm{U}(1)_{l} \subset \mathrm{SU}(2)_{l}$ and the $\mathrm{SU}(2 \mid 1)_{l}$ supersymetries

$$
\gamma_{3} \epsilon_{l}=\epsilon_{l} \quad \gamma_{3} \bar{\epsilon}_{l}=-\bar{\epsilon}_{l} .
$$

The Hopf circle, which is labeled by a point on $S^{2}$, also preserves $\mathrm{U}(1)_{r} \subset \mathrm{SU}(2)_{r}$. A Wilson loop supported on this Hopf circle preserves the following supersymmetries in $\mathrm{SU}(2 \mid 1)_{r}$

$$
\gamma_{3} \epsilon_{r}=\epsilon_{r} \quad \gamma_{3} \bar{\epsilon}_{r}=-\bar{\epsilon}_{r},
$$

where $\epsilon_{r}$ and $\bar{\epsilon}_{r}$ are non-constant spinors in the left-invariant frame. We can without loss of generality take the loop at the North or South pole of $S^{2}$, in which case $\mathrm{U}(1)_{r}$ is generated by $J_{3}^{r}$. For another Hopf circle at a different point in $S^{2}$, which can be obtained by the action of an isometry, the preserved $\mathrm{U}(1)_{r}$ and supersymmetry generators are obtained from those at the poles by conjugating by the action of the isometry.

In summary, the Wilson loop (5.8) wrapping the Hopf circle at the North pole of $S^{2}$ is half-supersymmetric in a $3 \mathrm{~d} \mathcal{N}=4$ theory on $S^{3}$. It preserves an $\mathrm{SU}(1 \mid 1)_{l} \times \mathrm{SU}(1 \mid 1)_{r}$ inside the $\mathrm{SU}(2 \mid 1)_{l} \times \mathrm{SU}(2 \mid 1)_{r}$ supersymmetry of a $3 \mathrm{~d} \mathcal{N}=4$ theory on $S^{3}$. The explicit supersymmetry algebra of the Wilson loop is

$$
\begin{aligned}
\left\{q_{l}, \bar{q}_{l}\right\} & =\frac{2}{L}\left[J_{3}^{l}-\frac{1}{2}\left(R_{C}+R_{H}\right)\right] \\
\left\{q_{r}, \bar{q}_{r}\right\} & =\frac{2}{L}\left[J_{3}^{r}-\frac{1}{2}\left(R_{C}-R_{H}\right)\right] .
\end{aligned}
$$

The insertion of this Wilson loop on $S^{3}$ can be also realized by coupling the $1 \mathrm{~d} \mathcal{N}=4$ fermi multiplet localized on the Hopf circle to the $3 \mathrm{~d} \mathcal{N}=4$ theory on $S^{3}$. Putting the $1 d$ gauged fermi multiplet theory on $S^{3}$ while preserving $\mathrm{SU}(1 \mid 1)_{l} \times \mathrm{SU}(1 \mid 1)_{r}$ is trivial as there are no $1 / L$ modifications to the $1 \mathrm{~d}$ gauged fermi theory in flat space. Integrating out the fermi multiplet inserts the Wilson loop operator (5.8).

\section{$5.2 \quad$ Vortex loops on $S^{3}$}

Another family of half-supersymmetric line operators can be defined in $3 \mathrm{~d} \mathcal{N}=4$ gauge theories on $S^{3}$. These loop operators preserve a different $\mathrm{SU}(1 \mid 1)_{l} \times \mathrm{SU}(1 \mid 1)_{r}$ inside the $\mathrm{SU}(2 \mid 1)_{l} \times \mathrm{SU}(2 \mid 1)_{r}$ supersymmetry on $S^{3}$. They correspond to Vortex loop operators. A Vortex loop operator supported on a Hopf circle at the North or South pole of $S^{3}$ preserves the following supersymmetries ${ }^{57}$

$$
\begin{aligned}
\gamma_{3} \epsilon_{l} & =\epsilon_{l} & \gamma_{3} \bar{\epsilon}_{l}=-\bar{\epsilon}_{l} \\
\gamma_{3} \epsilon_{r} & =-\epsilon_{r} & \gamma_{3} \bar{\epsilon}_{r}=\bar{\epsilon}_{r},
\end{aligned}
$$

Indeed the supersymmetry algebra preserved by a Vortex loop is different to the one preserved by a Wilson loop (cf. (5.13)(5.14)). We record in table 5 the quantum numbers of the preserved supercharges.

\footnotetext{
${ }^{57} \mathrm{~A}$ Vortex loop wrapping a different Hopf circle can be obtained by conjugating by a symmetry.
} 


\begin{tabular}{|c|c|c|c|c|}
\hline & $J_{3}^{l}$ & $J_{3}^{r}$ & $R_{C}$ & $R_{H}$ \\
\hline$q_{l}$ & $-1 / 2$ & 0 & $-1 / 2$ & $-1 / 2$ \\
$\bar{q}_{l}$ & $1 / 2$ & 0 & $1 / 2$ & $1 / 2$ \\
\hline$q_{r}$ & 0 & $1 / 2$ & $-1 / 2$ & $1 / 2$ \\
$\bar{q}_{r}$ & 0 & $-1 / 2$ & $1 / 2$ & $-1 / 2$ \\
\hline
\end{tabular}

Table 5. Supercharges preserving Vortex loop.

We note that $J_{3}^{l}-\frac{1}{2}\left(R_{C}+R_{H}\right)$ and $J_{3}^{r}+\frac{1}{2}\left(R_{C}-R_{H}\right)$ commute with all four supercharges, a fact will make good use of shortly. The explicit $\mathrm{SU}(1 \mid 1)_{l} \times \mathrm{SU}(1 \mid 1)_{r}$ anticommutators of the preserved supercharges by a Vortex loop operator are

$$
\begin{aligned}
\left\{q_{l}, \bar{q}_{l}\right\} & =\frac{2}{L}\left[J_{3}^{l}-\frac{1}{2}\left(R_{C}+R_{H}\right)\right] \\
\left\{q_{r}, \bar{q}_{r}\right\} & =\frac{2}{L}\left[J_{3}^{r}+\frac{1}{2}\left(R_{C}-R_{H}\right)\right] .
\end{aligned}
$$

Note the different relative sign in the last anticommutator in comparison with the supersymmetry algebra (5.16) for a Wilson loop.

In order to explicitly define Vortex loop operators on $S^{3}$ we must learn how to couple in an $\mathrm{SU}(1 \mid 1)_{l} \times \mathrm{SU}(1 \mid 1)_{r}$ invariant way the $1 \mathrm{~d} \mathcal{N}=4 \mathrm{SQM}_{V}$ invariant gauge theories discussed in section 2 to $3 \mathrm{~d} \mathcal{N}=4$ gauge theories on $S^{3}$. The $1 \mathrm{~d} \mathcal{N}=4$ theory is now supported on an $S^{1}$ and not on a line, modification which plays an important role in what follows. It is instructive to first note that in the flat space limit where $L \rightarrow \infty$ the supersymmetry preserved by the loop on $S^{3}$ reduces to the $1 \mathrm{~d} \mathcal{N}=4 \mathrm{SQM}_{V}$ supersymmetry in (2.3) that was preserved by a Vortex loop in flat space

$$
\begin{gathered}
\left\{q_{l}, \bar{q}_{l}\right\}=H \\
\left\{q_{r}, \bar{q}_{r}\right\}=-H,
\end{gathered}
$$

where the sphere generators $J_{3}^{l}=\frac{1}{2}\left(J_{12}+L H\right)$ and $J_{3}^{r}=\frac{1}{2}\left(J_{12}-L H\right)$ become the translation generator $H$ and transverse rotation generator $J_{12}$ in flat space.

Coupling a $1 \mathrm{~d} \mathcal{N}=4 \mathrm{SQM}_{V}$ gauge theory on the loop to a $3 \mathrm{~d} \mathcal{N}=4$ theory on $S^{3}$ requires turning on background fields in the $1 \mathrm{~d} \mathcal{N}=4$ theory in order to make the $3 \mathrm{~d} / 1 \mathrm{~d}$ theory invariant under the $\mathrm{SU}(1 \mid 1)_{l} \times \mathrm{SU}(1 \mid 1)_{r}$ supersymmetry algebra of the Vortex loop. This is not surprising. Placing a $3 \mathrm{~d} \mathcal{N}=4$ gauge theory on $S^{3}$ deforms the flat space supersymmetry transformations and action. Both modifications can be interpreted as arising from background $3 \mathrm{~d} \mathcal{N}=4$ supergravity fields [40]. We now show that the $1 \mathrm{~d}$ $\mathcal{N}=4$ supersymmetry transformations and action can be deformed in such a way as to yield a supersymmetric Vortex loop operator on $S^{3}$. 
The starting point is the $\mathrm{SQM}_{V}$ supersymmetry algebra discussed in section 2

$$
\left\{Q_{+}, \bar{Q}_{+}\right\}=H \quad\left\{Q_{-}, \bar{Q}_{-}\right\}=H .
$$

In order to couple the $1 \mathrm{~d} \mathcal{N}=4$ theory on $S^{3}$ supersymmetrically we must deform the $1 \mathrm{~d} \mathcal{N}=4$ theory in such a way that the supersymmetry algebra becomes the $\mathrm{SU}(1 \mid 1)_{l} \times$ $\mathrm{SU}(1 \mid 1)_{r}$ algebra of the Vortex loop on $S^{3}$

$$
\begin{aligned}
\left\{q_{l}, \bar{q}_{l}\right\} & =\frac{2}{L}\left[J_{3}^{l}-\frac{1}{2}\left(R_{C}+R_{H}\right)\right] \\
\left\{q_{r}, \bar{q}_{r}\right\} & =\frac{2}{L}\left[J_{3}^{r}+\frac{1}{2}\left(R_{C}-R_{H}\right)\right] .
\end{aligned}
$$

This can be accomplished in two steps:

- Turning on a fixed background gauge field for the R-symmetry $J_{-} \equiv J_{3}^{l}+J_{3}^{r}-R_{C}$

- Turning on a fixed imaginary mass parameter for the flavour symmetry $G_{F} \equiv J_{3}^{l}+$ $J_{3}^{r}-R_{H}$

$G_{F}$ does indeed commute with all four supercharges, as it is the sum of the bosonic generators in (5.19) and (5.20), both of which are central elements. The charge $J_{-}$commutes with $q_{l}$ and $\bar{q}_{l}$ but not with $q_{r}$ and $\bar{q}_{r}$. We also use in an important way that the $1 \mathrm{~d} \mathcal{N}=4$ theory lives on an $S^{1}$ and non-trivial Wilson lines can be turned on around the circle. Deforming the $1 \mathrm{~d} \mathcal{N}=4$ theory by these backgrounds allows to couple a $1 \mathrm{~d} \mathcal{N}=4$ theory on a Hopf circle to a $3 \mathrm{~d} \mathcal{N}=4$ theory on $S^{3}$ while preserving $\mathrm{SU}(1 \mid 1)_{l} \times \mathrm{SU}(1 \mid 1)_{r}$.

The $\mathrm{SQM}_{V}$ supersymmetry algebra (5.23) gets deformed in the presence of a background $1 \mathrm{~d} \mathcal{N}=4$ vector multiplet for a flavour symmetry $G$. We consider a background gauge field $a$ on $S^{1}$ and a background scalar field in the vector multiplet corresponding to a real mass $m .{ }^{58}$ The deformed $\mathrm{SQM}_{V}$ algebra becomes ${ }^{59}$

$$
\left\{Q_{+}, \bar{Q}_{+}\right\}=H-(a+i m) G \quad\left\{Q_{-}, \bar{Q}_{-}\right\}=H-(a-i m) G .
$$

$H$ is the generator of translations on the circle, which we take to have length $\beta=2 \pi L$.

Turning on a background gauge field for the R-symmetry $J_{-}$generically breaks the supersymmetries associated to the supercharged $Q_{-}$and $\bar{Q}_{-}$charged under $J_{-}$. However for quantized values of the gauge field $a_{-}=\frac{n}{L}, n \in \mathbb{Z}$, supersymmetry remains unbroken with $Q_{-}$and $\bar{Q}_{-}$generated by the non-constant Killing spinors $\epsilon_{-}=e^{i \frac{n \tau}{L}}$ and $\bar{\epsilon}_{-}=e^{-i \frac{n \tau}{L}}$, with $\tau \in[0,2 \pi L]$ parametrizing $S^{1}{ }^{60}$ For these quantized holonomies, the supersymmetry

\footnotetext{
${ }^{58}$ Turning on a complex mass associated to the other two scalars in the vector multiplet deforms other commutators.

${ }^{59}$ This can be easily understood by dimensional reduction of the $4 \mathrm{~d} \mathcal{N}=1$ supersymmetry algebra.

${ }^{60}$ This can be understood as follows. The constant gauge field $a_{-}$can be absorbed into a redefinition of the fields $\phi^{\prime}=e^{-i r_{-} a_{-} \tau} \phi$, with $r_{-}$the $J_{-}$-charge of $\phi$. The non-standard periodicity of the fields around $S^{1}$ are then preserved by the supersymmetry transformations generated with $\epsilon_{-}=e^{i a_{-} \tau}$ and $\bar{\epsilon}_{-}=e^{-i a_{-} \tau}$. These spinors are globally defined only for $a_{-}=\frac{n}{L}$, with $n \in \mathbb{Z}$.
} 
algebra is deformed in the same way as if it were a flavor symmetry. In conclusion, turning on the following background fields for $J_{-}$and $G_{F}$

$$
\begin{array}{cc}
J_{-}: a=-\frac{1}{L} & m=0 \\
G_{F}: a=0 & m=\frac{i}{L},
\end{array}
$$

the $\mathrm{SQM}_{V}$ algebra (5.23) becomes the $\mathrm{SU}(1 \mid 1)_{l} \times \mathrm{SU}(1 \mid 1)_{r}$ supersymmetry preserved by a line defect in a $3 \mathrm{~d} \mathcal{N}=4$ theory on $S^{3}$. We can identify the generators as follows: ${ }^{61}$

$$
\begin{array}{rlrl}
Q_{+} & =\bar{q}_{l}, & \bar{Q}_{+}=q_{l}, \quad Q_{-}=\bar{q}_{r}, & \bar{Q}_{-}=-q_{r} \\
H=-\frac{2}{L} J_{r}^{3}, & J_{ \pm}=\mp\left(J_{l}^{3}+J_{r}^{3}\right)-R_{C}, & G_{F}=J_{l}^{3}+J_{r}^{3}-R_{H}
\end{array}
$$

Consistently with the deformation by the R-symmetry gauge field $a_{-}=-\frac{1}{L}$, we find that the Killing spinors generating $q_{r}$ and $\bar{q}_{r}$ behave at the position of the loop as $\bar{\epsilon}_{-}=e^{\frac{i \tau}{L}}$ and $\epsilon_{-}=e^{-\frac{i \tau}{L}}$, respectively. Note that these position dependent Killing spinors are nevertheless periodic along the Hopf circle.

The background fields (5.27) ensure that the $1 \mathrm{~d} \mathcal{N}=4$ theory living on a maximal circle of $S^{3}$ can be coupled supersymmetrically to the bulk $3 \mathrm{~d} \mathcal{N}=4$ theory. This can also be understood by looking at the $3 \mathrm{~d} \mathcal{N}=4$ supersymmetry transformations of the vector multiplet and hypermultiplet on $S^{3}$ generated by the four Killing spinors preserving the Vortex loop. These transformations decompose into $\mathrm{SQM}_{V}$ supersymmetry transformations deformed by the advertised background. To couple the bulk theory to $\mathrm{SQM}_{V}$, preserving the four supercharges, it is then required to turn on this background in the $\mathrm{SQM}_{V} \mathrm{su}-$ persymmetry transformations and Lagrangian. The derivation of how the supersymmetry transformations on $S^{3}$ decompose is presented in appendix A.

The presence of these background fields can be recast, upon field redefinition, into twisted boundary conditions for the fields in the $1 \mathrm{~d} \mathcal{N}=4$ theory around $S^{1}$, thus affecting the partition function (or supersymmetric index) of the $1 \mathrm{~d} \mathcal{N}=4$ theory. This will play a crucial role in the evaluation of the expectation value of Vortex loop operators in section 5.3.

Having defined the half-supersymmetric Wilson and Vortex loop operators on $S^{3}$, we now propose to evaluate their expectation values exactly, using the results of supersymmetric localization and to test the mirror symmetry predictions of section 4 .

\subsection{Exact partition function of $3 \mathrm{~d} / 1 \mathrm{~d}$ theories on $S^{3}$}

In this section we identify the matrix integral representation of the exact expectation value of a half-supersymmetric Vortex (and Wilson) loop operator in an $\mathcal{N}=4$ theory on $S^{3}$, which takes into account the coupling of $1 \mathrm{~d} \mathcal{N}=4 \mathrm{SQM}$ on $S^{1}$ to the $\mathcal{N}=4$ theory on $S^{3}$ described in the previous section. This matrix model model is obtained by combining in an interesting way the matrix integral computing the $S^{3}$ partition function of $\mathcal{N}=4$ theory on $S^{3}[17]$ and the matrix model representation of the supersymmetric index of $1 \mathrm{~d}$ $\mathcal{N}=4$ SQM found in $[18,19]$.

\footnotetext{
${ }^{61}$ In the flat space limit, the R-symmetry generators $J_{ \pm}$are identified with $\mp J_{12}-R_{C}$. They are related to the generators $J_{ \pm}$presented in section 2 by a shift by the flavor symmetry generator $J_{12}-R_{H}$, which is the flat space limit of $G_{F}$.
} 


\subsection{1 $\quad S^{3}$ Partition function and Wilson loops}

A powerful probe of the dynamics of a strongly coupled $3 \mathrm{~d} \mathcal{N}=4$ IR SCFT emerging at the endpoint of an RG flow from a UV $3 \mathrm{~d} \mathcal{N}=4$ supersymmetric gauge theory is the partition function of the gauge theory on $S^{3}$. The $S^{3}$ partition function is a renormalization group invariant observable, and the computation performed in the UV exactly captures the partition function of the IR SCFT.

The $S^{3}$ partition function of $3 \mathrm{~d} \mathcal{N}=4$ gauge theories can be localized to a finite dimensional matrix integral [17]. The matrix integral is defined by integrating over the Cartan subalgebra of the gauge group the product of the classical and one-loop contributions in the (exact) saddle point analysis [17]

$$
Z_{M M}=\frac{1}{|\mathcal{W}|} \int_{C} d \sigma Z_{\text {class }} \cdot Z_{\text {vector }} \cdot Z_{\text {hyper }},
$$

where $\mathcal{W}$ is the Weyl group and $C$ is the contour of integration. The classical contribution depends on the FI parameter $\eta$ for each abelian gauge group factor

$$
Z_{\text {class }}=e^{2 \pi i \eta \operatorname{Tr} \sigma} \text {. }
$$

The $3 \mathrm{~d} \mathcal{N}=4$ vector multiplet contribution is

$$
Z_{\text {vector }}=\prod_{\alpha>0} \operatorname{sh}(\alpha \cdot \sigma)^{2}
$$

while a hypermultiplet in a representation $\mathcal{R}$ of the gauge group with mass $m$ yields

$$
Z_{\mathrm{hyper}}=\prod_{w \in \mathcal{R}} \frac{1}{\operatorname{ch}(w \cdot \sigma-m)},
$$

where $\alpha$ are the roots of the Lie algebra and $w$ the weights for the representation. Throughout we use the following short-hand notation (borrowed from [15])

$$
\operatorname{sh}(x) \equiv 2 \sinh (\pi x) \quad \operatorname{ch}(x) \equiv 2 \cosh (\pi x), \quad \operatorname{th}(x) \equiv \tanh (\pi x) .
$$

In this paper we focus on gauge theories with unitary gauge groups. For a $\mathrm{U}(N)$ gauge group the set of eigenvalues is $\left\{\sigma_{i}\right\}_{1 \leq i \leq N}$ and $|\mathcal{W}|=N$ !. The vector multiplet and massive fundamental hypermulptiplet factors are expressed in this case by

$$
Z_{\text {vector }}=\prod_{i<j} \operatorname{sh}\left(\sigma_{i}-\sigma_{j}\right)^{2}, \quad Z_{\text {hyper }}=\prod_{j=1}^{N} \operatorname{ch}\left(\sigma_{j}-m\right)^{-1} .
$$

By combining these building blocks the exact $S^{3}$ partition function of an arbitrary $3 \mathrm{~d}$ $\mathcal{N}=4$ gauge theory acquires an elegant matrix model representation.

The contour of integration $C$ of the matrix model that arises from the localization computation is the real axis. The condition that the matrix integral over the real axis is convergent [14] is precisely the same as the criterion for the quiver theory to be "good" or "ugly" [29], that is that for each $\mathrm{U}(N)$ gauge group factor the number of fundamental 

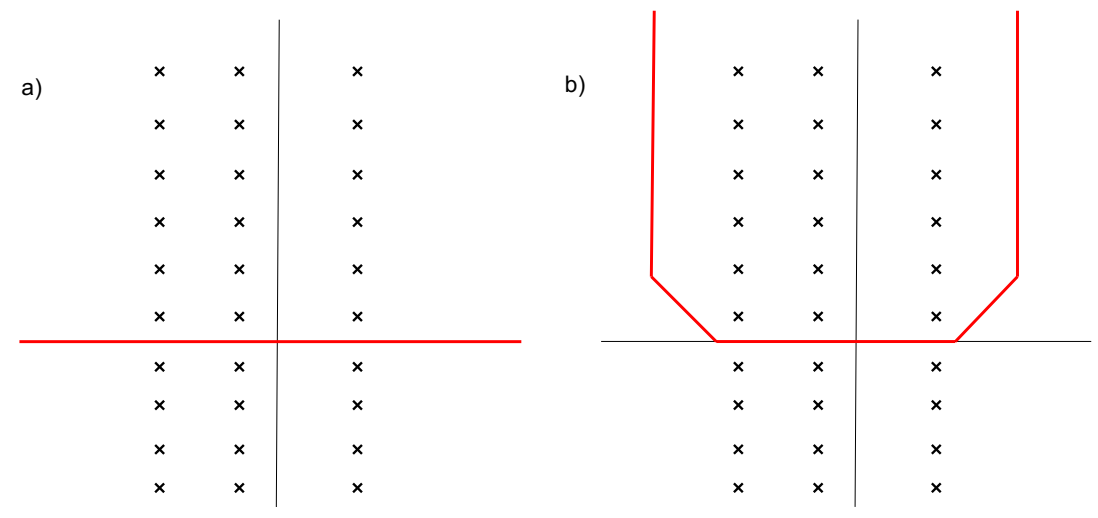

Figure 37. a) The naive contour (red line) of integration is on the real line. Crosses denote poles of the matrix integrand. b) At positive FI parameter, we deform the contour as depicted, so that it encloses the poles in the upper-half plane.

hypermultiplets $N_{f}$ obeys $N_{f} \geq 2 N-1$. In this paper we have considered gauge theories that flow in the IR to an irreducible SCFT, so the matrix models (in the absence of Wilson loops) are convergent.

The exact expectation value of the supersymmetric Wilson loop (5.8) is computed by inserting into the matrix model of the theory (5.30) the factor

$$
\operatorname{Tr}_{\mathcal{R}}\left(e^{2 \pi \sigma}\right)=\sum_{w \in \mathcal{R}} e^{2 \pi w \cdot \sigma}
$$

where $\mathcal{R}$ is the representation associated to the Wilson loop and $w$ runs over the weights of $\mathcal{R}$. Wilson loops expectation values are in addition normalized by the partition function.

We note that the matrix integrals defined over the real contour capturing the expectation value of Wilson loops can become divergent when the charge of the Wilson loop is sufficiently large. For these superficially divergent matrix models we regulate the divergent integrals by deforming the contour of integration $C$ of the matrix model away from the real axis. The results that we find using the deformed contours are completely consistent with our mirror symmetry predictions. It may be possible to justify these deformed contours of integration for the divergent matrix integrals by carefully performing the localization computation.

More precisely, for positive FI parameter $\eta>0$, we deform the contour of integration of each eigenvalue so that it encloses the poles of the matrix model integrand in the upper half-plane, as in figure 37. This is then the same as closing the real line with a semi-circle going through $+i \infty$. Conversely if $\eta<0$ we close the contour so that it encloses the poles in the lower half-plane. These choices of contour lead to finite results for arbitrary large values of the Wilson loop charge, as long as $\eta \neq 0$. For each matrix eigenvalue the Wilson loop charge $q$ combines with the FI parameter $\eta$ in a complex parameter $\eta+i q$ and the evaluation of the integral with our prescription coincides with the analytic continuation to the complex value of the FI parameter. 


\subsubsection{1d $\mathcal{N}=4$ Supersymmetric Quantum Mechanics partition function}

In this section we introduce and evaluate the partition function of the $1 \mathrm{~d} \mathcal{N}=4$ SQM quiver gauge theories on a circle relevant for the description of Vortex loop operators in $3 \mathrm{~d} \mathcal{N}=4$ gauge theories. The $1 \mathrm{~d} \mathcal{N}=4 \mathrm{SQM}$ partition function admits a Hilbert space interpretation as an index with fugacities (or chemical potentials). It is defined as a trace over the Hilbert space of the theory [45]

$$
\mathcal{I}(z, \mu)=\operatorname{Tr}_{\mathcal{H}}(-1)^{F} e^{2 \pi i z J_{-}} e^{2 \pi i \mu \Pi},
$$

where $F$ is the fermion number, $z$ is a chemical potential for the $\mathrm{U}(1)_{-}$R-symmetry with generator $J_{-}$and $\mu=\left\{\mu_{j}\right\}$ is a collection of chemical potentials for flavor symmetries with Cartan generators $\Pi_{j}$. In the path integral representation, the fugacities are associated to twisted boundary conditions for the fields, which can be undone by turning on background vector multiplets for the relevant symmetries. $z$ is associated to the presence of a $\mathrm{U}(1)_{-}$ R-symmetry gauge field, with the relation $z=-L a_{-}$, where $2 \pi L$ is the length of $S^{1}$. A chemical potential $\mu_{j}$ is associated to the presence of a $\mathrm{U}(1)_{j}$ flavor symmetry gauge field $a_{j}$ and a real mass parameter $m_{j}$, through the relation $\mu_{j}=-L\left(a_{j}+i m_{j}\right)$.

As explained in section 5.2, coupling the $1 \mathrm{~d} \mathcal{N}=4$ theory on a maximal $S^{1} \subset S^{3}$ to a $3 \mathrm{~d} \mathcal{N}=4$ theory requires turning on a constant R-symmetry gauge field corresponding to $z=1$ and a mass deformation corresponding to a chemical potential $\mu=1$ for the flavor symmetry generated by $G_{F}=J_{3}^{l}-J_{3}^{r}-R_{H}$. Of course, the fugacities for all other flavour symmetries are arbitrary.

The $1 \mathrm{~d} \mathcal{N}=4 \mathrm{SQM}$ theories are deformed by FI terms with parameters $\vec{\zeta} \equiv \zeta$, taking real values in the center of the gauge algebra. The partition function or index is welldefined when all FI parameters $\zeta_{a}$ are non-zero. When the FI parameters vanish important subtleties arise as the theory may develop a continuous spectrum.

Using supersymmetric localization, the exact index of a $1 \mathrm{~d} \mathcal{N}=4$ SQM theory is given by the explicit formula $[18,19]$

$$
\mathcal{I}(z, \mu)=\frac{1}{|W|} \mathrm{JK}-\operatorname{Res}_{\zeta} g_{\mathrm{vec}}(z, u) g_{\mathrm{chi}}(z, \mu, u) d^{k} u,
$$

where $k$ is the rank of the gauge group $G,|W|$ is the order of the Weyl group $W$ of $G$ and JK-Res denotes the sum over Jeffrey-Kirwan residues [46] of a meromorphic $k$-form with complex variables $u=\left\{u_{I}\right\}$. The factors $g_{\text {vec }}, g_{\text {chi }}$ are the localization one-loop determinants associated to $1 \mathrm{~d} \mathcal{N}=4$ vector and chiral multiplets,

$$
\begin{aligned}
g_{\mathrm{vec}}(z, u) & =\left(\frac{\pi}{\sin (\pi z)}\right)^{k} \prod_{\alpha \in G} \frac{\sin (-\pi \alpha \cdot u)}{\sin [\pi(\alpha \cdot u-z)]} \\
g_{\mathrm{chi}}(z, \mu, u) & =\prod_{w \in \mathcal{R}} \frac{\sin \left[-\pi\left(w \cdot u+q \cdot \mu+\left(\frac{R}{2}-1\right) z\right)\right]}{\sin \left[\pi\left(w \cdot u+q \cdot \mu+\frac{R}{2} z\right)\right]},
\end{aligned}
$$

where $\alpha$ runs over the roots of the gauge algebra, $w$ runs over the weights of the chiral multiplet representation, $R$ is the chiral multiplet R-charge under $2 J_{-}$and $q=\left\{q_{j}\right\}$ are the chiral multiplet charges under the flavor symmetry generators $\Pi=\left\{\Pi_{j}\right\}$. 
We now explain briefly how to compute the Jeffrey-Kirwan residues of the meromorphic $k$-form with simple poles and with FI parameters $\zeta$. A given set pole $u^{*}=\left\{u_{I}^{*}\right\}$ arises at the intersection of $k$ hyperplanes in $\mathbb{C}^{k}$, defined by the equations $w^{(I)} \cdot u^{*}+q^{(I)} \cdot \mu+\frac{R^{(I)}}{2} z=0,{ }^{62}$ with $I=1, \cdots, k$, appearing in the denominator of the chiral multiplet one-loop determinant. In principle we should also consider poles in the vector multiplet one-loop determinant but it happens that one cannot find a collection of $k$ hyperplanes intersecting at a point $u^{*}$ if one of the hyperplanes is described by $\alpha \cdot u-z=0 .{ }^{63}$ Thus each pole $u^{*}$ is associated with a set of $k$ weights $\left\{w^{(I)}\right\}$, each weight appearing in some chiral multiplet factor. A set of weights $\left\{w^{(I)}\right\}$ defines a cone $C\left(w^{(I)}\right)=\left\{\sum_{I}^{k} c_{I} w^{(I)} \mid c_{I}>0\right\} \subset \mathbb{R}^{k}$. The Jeffrey-Kirwan residue at $u^{*}$ is then given by

$$
\mathrm{JK}-\operatorname{Res}_{\zeta}\left[u^{*}\right] g(z, \mu, u) d^{k} u=\left\{\begin{array}{ll}
\operatorname{Res}\left[u^{*}\right] g(z, \mu, u) & \text { if }-\zeta \in C\left(w^{(I)}\right) \\
0 & \text { otherwise. }
\end{array},\right.
$$

where $\operatorname{Res}\left[u^{*}\right] g(z, \mu, u)$ denotes the usual residue at the pole $u=u^{*}$ and $\zeta$ is understood as a $k$-component vector.

The total index (5.38) is obtained by summing over all JK-residues at poles $u^{*}$

$$
\mathcal{I}(z, \mu)=\frac{1}{|W|} \sum_{u^{*}} \operatorname{JK}-\operatorname{Res}_{\zeta}\left[u^{*}\right] g_{\mathrm{vec}}(z, u) g_{\mathrm{chi}}(z, \mu, u) d^{k} u .
$$

We will give here the result of the evaluation of the index for the class of $1 \mathrm{~d} \mathcal{N}=4$ SQM quivers entering our construction of Vortex loop operators mirror to Wilson loops, relegating part of the details of the residue computations to appendix B.

The class of $1 \mathrm{~d} \mathcal{N}=4 \mathrm{SQM}$ quiver gauge theories we consider is described in figure 2 . They are linear quivers connected by pairs of bifundamental chiral multiplets, with $N_{L}$ fundamental and $N_{R}$ anti-fundamental chiral multiplets in the terminating node, which we mean to be the node on the right of the quiver in 2 (this is the one that is closest to the $3 \mathrm{~d}$ quiver in the combined $3 \mathrm{~d} / 1 \mathrm{~d}$ quiver). The gauge group is then $G=\prod_{p=1}^{P} \mathrm{U}\left(n_{p}\right)$, where $\mathrm{U}\left(n_{P}\right)$ denotes the terminating node. Moreover each node has either zero or one adjoint chiral multiplet and we denote $c_{p}^{\text {adj }} \in\{0,1\}$ the number of adjoint chiral multiplets in the $\mathrm{U}\left(n_{p}\right)$ node. As explained in section 3.2, the $1 \mathrm{~d}$ FI parameters of all nodes in the quiver are taken negative if the $1 \mathrm{~d} \mathcal{N}=4$ theory is read from the brane configuration with D1-strings moved to the closest NS5-brane on the right. Conversely they are all taken positive if the $1 \mathrm{~d} \mathcal{N}=4$ theory is read from the brane configuration with D1-strings moved to the closest NS5-brane on the left. ${ }^{64}$

\footnotetext{
${ }^{62}$ Strictly speaking the hyperplane equations are $w^{(I)} \cdot u^{*}+q^{(I)} \cdot \mu+\frac{R^{(I)}}{2} z=n$, with $n \in \mathbb{Z}$, but in the index computation only the hyperplane with $n=0$ should be picked.

${ }^{63}$ The pole from the vector multiplet is canceled by a corresponding zero from the chiral multiplet.

${ }^{64}$ Less restrictive conditions could be imposed on the signs of the FI parameters in non-terminating nodes. For instance in a two-node quiver with FI parameter $\zeta_{2}>0$ in the terminating node, we could allow for a FI parameter $\zeta_{1}>-\zeta_{2}$, with $\zeta_{1} \neq 0$, in the other node. This constraint follows from a careful analysis of the positions of the various NS5-branes in the brane realization of the loop operator. We checked with a few explicit computations that the index does not depend on $\zeta_{1}$ taking values in this range. At $\zeta_{1}=0$, corresponding to aligned NS5-branes, we do not know how to evaluate the $1 \mathrm{~d}$ partition function, but we expect a dramatic change in the result (see discussion around 3.1). To simplify computations we require the FI parameters of all the nodes in a quiver to have the same sign $\left(\zeta_{1}, \zeta_{2}>0\right.$ in the example).
} 
Superpotentials. The $1 \mathrm{~d} \mathcal{N}=4 \mathrm{SQM}$ theories have superpotentials constraining the R-charges of the chiral multiplets: cubic superpotential couplings between adjoint and bifundamental chiral multiplets and quartic superpotential couplings between bifundamental chiral multiplets for the nodes without adjoint chiral multiplet. The $N$ fundamental and $M$ anti-fundamental chiral multiplets in the terminating $\mathrm{U}\left(N_{P}\right)$ node do not enter into such cubic and quartic superpotentials. Instead they are coupled to a $3 \mathrm{~d}$ hypermultiplet through a cubic superpotential as described in section 3.2. This $3 \mathrm{~d} / 1 \mathrm{~d}$ coupling is responsible for the identification of bulk and defect flavor symmetries.

A cubic superpotential imposes the constraint $R_{\mathrm{adj}}+R_{\mathrm{bif} 1}+R_{\mathrm{bif} 2}=2$ on the $\mathrm{R}$ charges of the fields with respect to the generator $2 J_{-}$. A quartic superpotential imposes the constraint $R_{\mathrm{bif} 1}+R_{\mathrm{bif} 2}+R_{\mathrm{bif} 3}+R_{\mathrm{bif} 4}=2$. The cubic superpotential which couples the fundamental and anti-fundamental chirals to the $3 \mathrm{~d}$ hypermultiplet imposes the constraints $R_{\text {fund }}+R_{\mathrm{a}-\text { fund }}=2$, since the bulk hypermultiplet is not charged under $J_{-}=J_{l}^{3}+J_{r}^{3}-R_{C}$.

In order to perform the computation of the Jeffrey-Kirwan residues, we only impose that the R-charges obey these superpotential constraints, but otherwise leave them arbitrary, to avoid having to deal with higher order poles. It turns out that the final results depend only on the R-charges of the adjoint chiral multiplets, which we need to specify at the end of the computation.

The R-charges for the adjoint chiral multiplets can be read from the flat space brane realization of the quiver (see section 3.2). The complex scalar degrees of freedom of an adjoint chiral living on D1-branes stretched between two NS5-branes correspond to displacements of the D1-branes along the plane $x^{1}-x^{2}$. The scalar transforms as a vector under $\mathrm{SO}(2)_{12}$ and is uncharged under $J_{78}$. The adjoint chiral then has charge 2 under $2\left(J_{12}-J_{78}\right)$ which is identified with the $1 \mathrm{~d} \mathcal{N}=4 \mathrm{SQM}$ generator $2 J_{-}$in the flat space limit. On the other hand the complex scalar of adjoint chiral living on D1-branes stretched between two NS5'-branes are associated with displacements of the D1-branes along the plane $x^{4}-x^{5}$. It transforms as a vector under $\mathrm{SO}(2)_{45}$ and is uncharged under $J_{12}$ and $J_{78}$. The adjoint chiral is then uncharged under $2 J_{-}$.

The R-charges (under $2 J_{-}$) of the adjoint chiral multiplets are then fixed to be either 2 or 0 , depending on whether they arise from a brane construction with NS5 or NS5' branes. In the $1 \mathrm{~d} \mathcal{N}=4$ quiver gauge theory description, this prescription is re-expressed as follows. Define the integers $\mathbf{c}_{p}=P-p-\sum_{q=p}^{P} c_{q}^{\text {adj }}$. An adjoint chiral multiplet in the $\mathrm{U}\left(n_{p}\right)$ node has R-charge $R_{\mathrm{adj}, p}$ given by

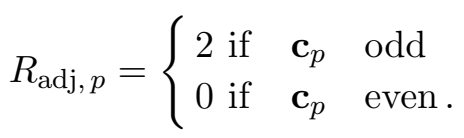

Similarly one can derive the charge of the adjoint chiral multiplets under the flavor symmetry $G_{F}$, which is identified in the flat space limit with the $10 \mathrm{~d}$ rotation generator $J_{12}-J_{45}$. The charges of the multiplets under the symmetries relevant for the computation of the index and the constraints they obey are summarized in table 6 .

The last entry in the table, $q_{+}+q_{-}=1 / 2$, follows from the superpotential constraint coupling bulk and defect fields and the fact that the $3 \mathrm{~d}$ hypermultiplet entering in this superpotential has charge $-1 / 2$ under $G_{F}=J_{l}^{3}+J_{r}^{3}-R_{H}$. 


\begin{tabular}{|c|c|c|c|}
\hline & $2 J_{-}$ & $G_{F}$ & W-constraints \\
\hline adjoint chiral & 2 or 0 & +1 or -1 & cubic \\
bifund. chiral & $r_{\text {bif }}$ & $q_{\text {bif }}$ & cubic/quartic sp. \\
fund. chiral & $r_{+}$ & $q_{+}$ & \\
antifund. chiral & $r_{-}$ & $q_{-}$ & $r_{+}+r_{-}=2, q_{+}+q_{-}-1 / 2=0$ \\
\hline
\end{tabular}

Table 6. Charges and superpotential constraints.

As explained in section 3.2 , each $1 \mathrm{~d} \mathcal{N}=4$ quiver gauge theory obtained by moving the D1-branes to the closest NS5-brane on the right is associated to a tensor product representation $\mathcal{R}$ of $\mathrm{U}(N)$, with $N$ the number of fundamental chiral multiplets, which depends on the ranks of the nodes $n_{p}$ and on the distribution of adjoint chiral multiplets among the nodes. The representation of $\mathrm{U}(N)$ associated to the quiver is $\mathcal{R}=\otimes_{p=1}^{P} \mathcal{R}_{k_{p}}$ with $k_{p}=n_{p}-n_{p-1}\left(\right.$ with $\left.n_{0}=0\right)$ and $\mathcal{R}_{k_{p}}$ is given by

$$
\mathcal{R}_{k_{p}}=\left\{\begin{array}{llll}
\mathcal{S}_{k_{p}} & \text { if } & \mathbf{c}_{p} & \text { odd } \\
\mathcal{A}_{k_{p}} & \text { if } & \mathbf{c}_{p} & \text { even }
\end{array}\right.
$$

$\mathcal{S}_{k_{p}}$ and $\mathcal{A}_{k_{p}}$ denote the $k_{p}$-symmetric representation and $k_{p}$-antisymmetric representation respectively.

Once the representation $\mathcal{R}$ is identified we can perform the evaluation of the $1 \mathrm{~d} \mathcal{N}=4$ SQM index. As we will explain shortly, we first compute the index at arbitrary values of $z$ and then we take the analytic continuation to $z \rightarrow 1$ in the combined $3 \mathrm{~d} / 1 \mathrm{~d}$ partition function. ${ }^{65}$ This turns out to be the correct prescription to compute the final exact partition function of the $3 \mathrm{~d} / 1 \mathrm{~d}$ theory describing a Vortex loop operator. The $G_{F}$ chemical potential $\mu$ can be readily set to $\mu=1$.

The details of the computations are given in appendix B, for single node and two nodes quivers. We use the explicit results of our computations to conjecture the index formula for the general class of quivers relevant in this paper. Our computations in explicit examples gives us confidence in our formulas (5.45)(5.47) below and ultimately result in the matching between mirror and Vortex loops extended to an arbitrary number of nodes.

Let us denote $\mathcal{I}^{r}$ and $\mathcal{I}^{l}$ the partition functions, or supersymmetric indices, of the $1 \mathrm{~d}$ $\mathcal{N}=4$ gauge theory realized by moving the D1-strings on the closest NS5-brane on the right and on the left respectively. For the 'right' $\mathrm{SQM}_{V}$ theory, which has $N$ fundamentals with mass $-\sigma_{j}$ and $M$ anti-fundamentals of mass $m_{a}$ in the terminating node, we find that the index $\mathcal{I}^{r}$ at arbitrary $z$ is given by a sum of contributions labeled by the weights of the representation $\mathcal{R}$ of $\mathrm{U}(N)$ :

$$
\mathcal{I}^{r}(z)=\sum_{w \in \mathcal{R}} \mathcal{I}_{w}^{r}(z), \quad \mathcal{I}_{w}^{r}(z)=\mathcal{F}\left(\sigma_{i}, z\right) \prod_{a=1}^{M} \prod_{j=1}^{N} \frac{\operatorname{ch}\left(m_{a}-\sigma_{j}\right)}{\operatorname{ch}\left(m_{a}-\sigma_{j}-i w_{j} z\right)}
$$

\footnotetext{
${ }^{65}$ Poles of the 3 d integrand cross the integration contour as $z \rightarrow 1$, which is why we analytically continue to $z=1$.
} 
where $\mathcal{F}\left(\sigma_{i}, z\right)$ is a factor that will not play any role in our final computation of the expectation value of Vortex loop operators. Let us just mention here that it is a product of the form

$$
\mathcal{F}\left(\sigma_{i}, z\right)=\prod_{\alpha, \kappa} \prod_{i \neq j}^{N} \frac{\sin \left[-\pi\left(i \sigma_{i}-i \sigma_{j}+\alpha z \pm z+\kappa / 2\right)\right]}{\sin \left[\pi\left(i \sigma_{i}-i \sigma_{j}+\alpha z+\kappa / 2\right)\right]}
$$

where $\alpha, \kappa$ take real values. In the analytic continuation to $z=1$, this factor will not affect the evaluation of the coupled $3 \mathrm{~d} / 1 \mathrm{~d}$ matrix model.

For the $1 \mathrm{~d} \mathcal{N}=4$ quiver theory obtained by moving the D1-branes to the closest NS5brane on the left the computation is similar, except for the fact that the FI parameters of the nodes are all positive. This affects very significantly the index. The answer is now written as a sum over weights of a representation $\mathcal{R}$ of $\mathrm{U}(N)$, where now $N$ is the number of anti-fundamental chiral multiplets in the terminating node and $\mathcal{R}$ is given by the same tensor product of symmetric and anti-symmetric representation described above. Denoting by $-\widetilde{m}_{a}$ the mass of the $\widetilde{M}$ fundamental chiral multiplets and $\sigma_{j}$ the mass of the $N$ anti-fundamental chiral multiplets, the index is then given by

$$
\mathcal{I}^{l}(z)=\sum_{w \in \mathcal{R}} \mathcal{I}_{w}^{l}(z), \quad \mathcal{I}_{w}^{l}(z)=\widetilde{\mathcal{F}}\left(\sigma_{i}, z\right) \prod_{a=1}^{\widetilde{M}} \prod_{j=1}^{N} \frac{\operatorname{ch}\left(\widetilde{m}_{a}-\sigma_{j}\right)}{\operatorname{ch}\left(\widetilde{m}_{a}-\sigma_{j}+i w_{j} z\right)}
$$

where $\widetilde{\mathcal{F}}\left(\sigma_{i}, z\right)$ is again a factor that trivializes when we consider the full $3 \mathrm{~d} / 1 \mathrm{~d}$ matrix model in the analytic continuation to $z=1$.

The formulae (5.45) and (5.47) are compatible with the expectations from 1d Seiberg duality. This can be understood as follows. The computation shows that the partition function depends only on the representation $\mathcal{R}$ and on the masses of the fundamental and anti-fundamental chiral multiplets in the terminating node. Multiple 1d quivers (except for single node quivers) lead to the same tensor product representation $\mathcal{R}$ and hence have equal partition functions, namely the quivers read from all possible orderings of the NS5 and NS5'-branes in the $x^{6}$ direction. These reorderings affect the rank of the gauge nodes and the distribution of adjoint chiral multiplets among them but not the associated representation $\mathcal{R}$. These Seiberg-dual quivers are expected to realize the same Vortex loop in the infrared limit and we find indeed that their partition functions match (at least when $z \rightarrow 1)$.

\subsubsection{Matrix model computing Vortex loops}

Our aim is to compute the exact expectation value of Vortex loops in $3 \mathrm{~d} \mathcal{N}=4$ gauge theories on $S^{3}$. We now explain how this quantity is captured by a matrix model that combines in an interesting way the exact $S^{3}$ partition of $3 \mathrm{~d} \mathcal{N}=4$ theories and the exact index of the $1 \mathrm{~d} \mathcal{N}=4$ theories defining Vortex loops, both of which we have already introduced.

A Vortex loop on $S^{3}$ is defined by a supersymmetric coupling of a $3 \mathrm{~d} \mathcal{N}=4$ gauge theory on $S^{3}$ to a $1 \mathrm{~d} \mathcal{N}=4$ gauge theory on an $S^{1} \subset S^{3}$. Recall that in order for the $3 \mathrm{~d} / 1 \mathrm{~d}$ defect theory to be supersymmetric, invariant under $\mathrm{SU}(1 \mid 1)_{l} \times \mathrm{SU}(1 \mid 1)_{r}$, we had to turn on 
very specific background fields in the SQM. We can compute the exact partition function of the combined $3 \mathrm{~d} / 1 \mathrm{~d}$ theory by choosing the supercharge $q_{l}$ in $\mathrm{SU}(1 \mid 1)_{l}$ to localize the full functional integral. In order to localize the combined $3 \mathrm{~d} / 1 \mathrm{~d}$ partition function, we must add suitable deformation terms for both the $3 \mathrm{~d} \mathcal{N}=4$ theory and the $1 \mathrm{~d} \mathcal{N}=4$ theory. Importantly, the saddle points for the $3 \mathrm{~d}$ and $1 \mathrm{~d}$ fields are not modified. The way the combined $3 \mathrm{~d} / 1 \mathrm{~d}$ partition function encodes the specific couplings between the $3 \mathrm{~d}$ and $1 \mathrm{~d}$ theories is by taking into account that the flavour symmetries of the $1 \mathrm{~d} \mathcal{N}=4$ theory are gauged with $3 \mathrm{~d} \mathcal{N}=4$ vector multiplets. At the level of the combined matrix model this means that the $1 \mathrm{~d}$ mass parameters that are gauged are replaced by the scalar in the corresponding $3 \mathrm{~d} \mathcal{N}=4$ vector multiplet. This scalar can be either dynamical, in which case it corresponds to an eigenvalue in the $3 \mathrm{~d}$ matrix integral that needs to be integrated over, or it is identified with a mass parameter in the $3 \mathrm{~d}$ theory. Therefore, the combined $3 \mathrm{~d} / 1 \mathrm{~d}$ partition function is found by convolving the "gauged" $1 \mathrm{~d}$ partition function with the $3 \mathrm{~d}$ partition function.

The expectation value of a Vortex loop $V$ wrapping a maximal circle of $S^{3}$ is the partition function of the $3 \mathrm{~d} \mathcal{N}=4$ theory coupled to the $1 \mathrm{~d} \mathcal{N}=4 \mathrm{SQM}$ quiver, normalized by the partition function of the $3 \mathrm{~d}$ theory. It takes the form

$$
\langle V\rangle=W^{\mathrm{fl}} \times \frac{Z_{3 d / 1 d}}{Z_{3 d}}=W^{\mathrm{fl}} \times \frac{1}{Z_{3 d}} \lim _{z \rightarrow 1} \frac{1}{|\mathcal{W}|} \int_{C} d \sigma F_{3 \mathrm{~d}}[\sigma, m, \xi] \mathcal{I}[\sigma, m, z] .
$$

$F_{3 \mathrm{~d}}[\sigma, \xi, m]$ is the matrix model integrand of a $3 \mathrm{~d} \mathcal{N}=4$ theory with eigenvalues $\sigma$, hypermultiplet masses $m$ and FI parameters $\xi$, while $\mathcal{I}[\sigma, m, z]$ is the index of the $1 \mathrm{~d} \mathcal{N}=4$ $\mathrm{SQM}_{V}$ defect theory that realizes the Vortex loop. We note that the flavour fugacities of the $1 \mathrm{~d}$ theory are identified either with $3 \mathrm{~d}$ matrix eigenvalues $\sigma$ or with $3 \mathrm{~d}$ mass parameters $m$, depending on whether the corresponding $1 \mathrm{~d}$ flavour symmetry is gauged by dynamical or background $3 \mathrm{~d}$ vector multiplets.

The factor $W^{\mathrm{fl}}$ takes the form of an abelian background Wilson loop that we add to the matrix model. It follows from the analysis of the brane realization of the Vortex loop and appears as a necessary ingredient to check successfully mirror maps with Wilson loops. In the brane picture each five-brane is associated to a deformation parameter of the theory (see section 3), masses $m_{a}$ for D5-branes and "FI" parameters $\xi_{a}$ for NS5-branes. When $q$ F1-strings end on a D5 with parameter $m$, they realize a Wilson loop of charge $q$ for the flavor symmetry associated to the D5-brane, and the corresponding matrix model factor is $e^{2 \pi q m}$. Similarly when $q$ D1-branes end on an NS5-brane with parameter $\xi$, they realize a Wilson loop of charge $q$ for the global symmetry associated to the NS5-brane, and the corresponding matrix model factor is $e^{2 \pi q \xi}$. Thus when we read the $3 \mathrm{~d} / 1 \mathrm{~d}$ quiver for a Vortex loop by moving the D1-branes on top of the closest NS5-brane to the left, resp. to the right, we propose to add to the matrix model the background Wilson loop factor

$$
\text { left: } \quad W^{\mathrm{fl}}=e^{2 \pi|\mathcal{R}| \xi_{L}}, \quad \underline{\text { right: }} \quad W^{\mathrm{fl}}=e^{2 \pi|\mathcal{R}| \xi_{R}},
$$

where $\xi_{L}$, resp. $\xi_{R}$, is the parameter associated to the NS5-brane on the left, resp. on the right, and $|\mathcal{R}|$ is the total number of boxes in the Young tableau of the representation $\mathcal{R}$ labeling the Vortex loop. $|\mathcal{R}|$ coincides with the total number of D1-branes ending on 
the NS5-brane. It should be noticed that this Wilson loop factor cannot be associated to a global symmetry acting on the fields of the theory, since the parameter $\xi_{L / R}$ is not a combination of the true FI parameters $\xi_{a}-\xi_{a+1}$. With this addition, the matrix model computing the Vortex loop depends on all $\xi_{a}$ parameters. Similarly the matrix model computing the Wilson loop depends on all the masses $m_{a}$ of fundamental hypermultiplets, although only the parameters $m_{a}-m_{a+1}$ are associated to actual flavors symmetries. ${ }^{66} \mathrm{In}$ the presence of a loop operator, the theory seems to admit one extra deformation parameter, mass or FI parameter, associated to a "hidden" symmetry, which act on the fields trivially.

A Vortex loop $V_{M, \mathcal{R}}^{(N)}$ coupled to a $\mathrm{U}(N)$ node of the $3 \mathrm{~d} \mathcal{N}=4$ quiver theory is labeled by a representation $\mathcal{R}$ of $\mathrm{U}(N)$ and by a splitting $K=M+(K-M)$ of the $K$ fundamental hypermultiplets of this $\mathrm{U}(N)$ gauge node. Let us consider the Vortex loop realization furnished by coupling the $3 \mathrm{~d} \mathcal{N}=4$ theory to the $1 \mathrm{~d} \mathcal{N}=4$ SQM quiver obtained by moving the D1-branes to the NS5-brane on the right, illustrated in figure 5. In this case the SQM is labeled by a certain representation $\mathcal{R}$ of $\mathrm{U}\left(N_{1}\right)$ and a splitting $M=M_{2}$, $K-M=M_{1}$. In the terminating node, the SQM has $N_{1}$ fundamental chiral multiplets with masses $-\sigma_{j}, j=1, \cdots, N_{1}$ and $M_{2}+N_{2}$ anti-fundamental chiral multiplets with masses $m_{a}, a=1, \cdots, M_{2}$ and $\widetilde{\sigma}_{k}, k=1, \cdots, N_{2}$. The couplings to the bulk theory identify $\sigma_{j}$ with the eigenvalues of the $3 \mathrm{~d} \mathrm{U}\left(N_{1}\right)$ gauge node, $\widetilde{\sigma}_{k}$ with the eigenvalues of the $3 \mathrm{~d} \mathrm{U}\left(N_{2}\right)$ gauge node and $m_{a}$ with the real masses of the $M_{2}$ fundamental bulk hypermultiplets. The identification of bulk and defect parameters works similarly for the SQM obtained by moving the D1-branes to the left NS5-brane.

We must also explain what we mean by $\lim _{z \rightarrow 1}$. The evaluation of (5.48) at imaginary values of $z$ defines a function that admits an analytic continuation to the complex plane. We can thus evaluate the integral for $z \in i \mathbb{R}$ and consider the analytic continuation to $z=1$. $^{67}$ This is how we extract our final result. This recipe is motivated by the observation that plugging $z=1$ directly in the matrix model integrand would trivialize the index (reduce it to be just one) and the coupling to the SQM quiver would not affect at all the total partition function. Instead, the analytic continuation that we propose takes into account the matrix model contributions from residues of poles crossing the $\sigma_{i}$-integration contours, as we vary $z$ continuously from zero to one. The fact that these residues should be included should follow from a more detailed analysis of the localization computation.

We can now explain our claim that the factor $\mathcal{F}\left(\sigma_{i}, z\right)$ appearing in the evaluation of the SQM index (5.45) does not contribute to $\left\langle V_{M, \mathcal{R}}^{(N)}\right\rangle$. We noticed that $\mathcal{F}\left(\sigma_{i}, z\right)$ is a product of terms with the generic form (5.46). In taking the analytic continuation $z \rightarrow 1$ these factors introduce residues from poles crossing the integration contours proportional to $\sin ( \pm \pi z)$. In the limit $z=1$ these extra residues all vanish, implying that the limit $z=1$ can be taken directly in the integrand of (5.48) for this factor $\mathcal{F}\left(\sigma_{i}, z\right)$. Noting that $\mathcal{F}\left(\sigma_{i}, z=1\right)= \pm 1$, we can simply drop this factor from our computations. Our results will be valid only up to an overall sign.

\footnotetext{
${ }^{66}$ This is because the sum of all fundamental hypermultiplet masses is associated to the diagonal U(1) of the flavor symmetry group, which is actually gauged with a dynamical gauge field.

${ }^{67}$ We note that as we move $z \in i \mathbb{R}$ no poles cross the integration contour, while if $z \in \mathbb{R}$ we must deform the contour to enclose the poles that cross the original one.
} 
The same argument would not work for the other factors in (5.45) because the numerators $\operatorname{ch}\left(m_{a}-\sigma_{j}\right)$ are exactly canceled by inverse factors from the $3 \mathrm{~d}$ hypermultiplets contributions! This means that the residues of the poles crossing the integration contour as $z \rightarrow 1$ due to these factors are non-vanishing. The discussion is the same for the left index (5.47).

\subsection{Loops in $T[\mathrm{SU}(N)]$}

In this section we perform the explicit computation of the exact expectation value of Wilson and Vortex loop operators in the $T[\mathrm{SU}(N)]$ theory. We show precise agreement with all of our brane-based predictions in section 4.3.

We will use the notations

$$
\prod_{j}^{a} \equiv \prod_{j=1}^{a}, \quad \prod_{j<k}^{a} \equiv \prod_{1 \leq j<k \leq a}, \quad \prod_{j, k}^{a, b} \equiv \prod_{j=1}^{a} \prod_{k=1}^{b} .
$$

The $T[\mathrm{SU}(N)]$ theory has an $\mathrm{U}(N)_{J} \times \mathrm{U}(N)_{F}$ global symmetry group. ${ }^{68} \mathrm{SU}(N)_{F}$ is the flavor symmetry rotating the fundamental hypermultiplets and $\mathrm{SU}(N)_{J}$ is a global symmetry arising at the infrared fixed point, enhancing the manifest $\mathrm{U}(1)^{N-1}$ topological symmetry acting on the dual photons. These global symmetries can be weakly gauged to give masses $m=\left(m_{1}, \ldots, m_{N}\right)$ to the fundamental hypermultiplets and FI parameters $\eta_{i}=\xi_{i}-\xi_{i+1}$, with $\xi=\left(\xi_{1}, \ldots, \xi_{N}\right)$, to the $N-1$ nodes. The $S^{3}$ partition function of the $T[\mathrm{SU}(N)]$ theory deformed by these massive parameters was computed in $[15,47]$ and is given by

$$
Z^{T[\mathrm{SU}(N)]}=(-i)^{\frac{N(N-1)}{2}} e^{-2 i \pi \xi_{N} \sum_{j} m_{j}} \frac{\sum_{\tau \in S_{N}}(-1)^{\tau} e^{2 \pi i \sum_{j}^{N} \xi_{j} m_{\tau(j)}}}{\prod_{j<k}^{N} \operatorname{sh}\left(\xi_{j}-\xi_{k}\right) \operatorname{sh}\left(m_{j}-m_{k}\right)},
$$

where $S_{N}$ is the group of permutations of $N$ elements and $(-1)^{\tau}$ is the signature of the permutation $\tau . T[\mathrm{SU}(N)]$ has the property of being self-mirror. This is realized by virtue of the partition function (5.51) being invariant under the exchange $m_{j} \leftrightarrow \xi_{j}$, except for the phase $e^{-2 i \pi \xi_{N} \sum_{j} m_{j}}$, which is unphysical, in the sense that it can be removed by a local counterterm constructed from the background fields (namely mixed background ChernSimons terms) defining the UV partition function [48, 49].

For the explicit mirror symmetry maps, it will prove useful to define the mirror symmetric quantity

$$
\mathcal{Z}^{T[\mathrm{SU}(N)]} \equiv e^{2 i \pi \xi_{N} \sum_{j} m_{j}} Z^{T[\mathrm{SU}(N)]}=(-i)^{\frac{N(N-1)}{2}} \frac{\sum_{\tau \in S_{N}}(-1)^{\tau} e^{2 \pi i \sum_{j}^{N} \xi_{j} m_{\tau(j)}}}{\prod_{j<k}^{N} \operatorname{sh}\left(\xi_{j}-\xi_{k}\right) \operatorname{sh}\left(m_{j}-m_{k}\right)},
$$

which can be understood as the partition function expressed in another renormalization scheme and which is manifestly invariant under the exchange of mass and FI parameters $m_{j} \leftrightarrow \xi_{j}$

\footnotetext{
${ }^{68}$ Strictkly speaking the symmetry is $\mathrm{SU}(N)_{J} \times \mathrm{SU}(N)_{F}$, but it is convenient to make the mapping of parameters under mirror symmetry symmetric to add two extra $\mathrm{U}(1)$ symmetries, which act trivially.
} 
All our results are nicely expressed in terms of two shift operators $\mathfrak{S}^{\mathbf{q}}$ and $\widehat{\mathfrak{S}}^{\mathbf{q}}$ acting on the partition function $Z \equiv Z^{T[\mathrm{SU}(N)]}$ of the theory. These operators act by shifting respectively the FI parameters and the masses by imaginary terms,

$$
\begin{array}{ll}
\mathfrak{S}^{\mathbf{q}} Z \equiv Z_{\xi_{j} \rightarrow \xi_{j}-i q_{j}}, & j=1, \cdots, N, \\
\widehat{\mathfrak{S}}^{\mathbf{q}} Z \equiv Z_{m_{j} \rightarrow m_{j}-i q_{j}}, & j=1, \cdots, N,
\end{array}
$$

where $\mathbf{q}=\left(q_{1}, \cdots, q_{N}\right)$ is a $N$-component vector.

\subsubsection{T $T[\mathrm{SU}(2)]$ loops}

Let us start our analysis with the abelian theory $T[\mathrm{SU}(2)]$. The partition function is given by the matrix model

$$
Z=\int d \sigma \frac{e^{2 i \pi\left(\xi_{1}-\xi_{2}\right) \sigma}}{\operatorname{ch}\left(\sigma-m_{2}\right) \operatorname{ch}\left(\sigma-m_{1}\right)} .
$$

One can evaluate this integral by closing the contour of integration by a semi-circle going through $i \infty$ (or $-i \infty)$ and summing over the residues inside the contour. This yields

$$
Z=(-i) e^{-2 i \pi \xi_{2}\left(m_{1}+m_{2}\right)} \frac{e^{2 i \pi\left(\xi_{1} m_{1}+\xi_{2} m_{2}\right)}-e^{2 i \pi\left(\xi_{1} m_{2}+\xi_{2} m_{1}\right)}}{\operatorname{sh}\left(\xi_{1}-\xi_{2}\right) \operatorname{sh}\left(m_{1}-m_{2}\right)}
$$

which agrees with (5.51) for $N=2$.

The matrix model computing the vev of a Wilson loop of charge $q \in \mathbb{Z}$ is given by

$$
\begin{aligned}
\left\langle W_{q}\right\rangle & =\frac{1}{Z} \int d \sigma \frac{e^{2 i \pi\left(\xi_{1}-\xi_{2}\right) \sigma}}{\operatorname{ch}\left(\sigma-m_{2}\right) \operatorname{ch}\left(\sigma-m_{1}\right)} e^{2 \pi q \sigma}=\frac{\mathfrak{S}^{(q, 0)} Z}{Z} \\
& =(-1)^{q} \frac{e^{2 \pi q m_{1}} e^{2 i \pi\left(\xi_{1} m_{1}+\xi_{2} m_{2}\right)}-e^{2 \pi q m_{2}} e^{2 i \pi\left(\xi_{1} m_{2}+\xi_{2} m_{1}\right)}}{e^{2 i \pi\left(\xi_{1} m_{1}+\xi_{2} m_{2}\right)}-e^{2 i \pi\left(\xi_{1} m_{2}+\xi_{2} m_{1}\right)}},
\end{aligned}
$$

where we evaluated the integral at non-zero $q$ by the analytical continuation $\xi_{1} \rightarrow \xi_{1}-i q$, following from our choice of deformed contour integral (see discussion after (5.36)). ${ }^{69}$

Let us turn now to the computation of Vortex loops. The $T[\mathrm{SU}(2)]$ theory has two fundamental hypermultiplets, which give rise to three possible splitting $2=i+(2-i)$, $i=0,1,2$, defining three possible Vortex loops $V_{i, q}$. Using the result for the Vortex loop factor (5.45), computed from its right SQM quiver realization (and ignoring the factor $\mathcal{F}$ for the reasons mentioned before), inserted in (5.48) and with extra Wilson line (5.49), the matrix models computing the Vortex loop vevs are found to be $\mathrm{e}^{70}$

$$
\begin{aligned}
\left\langle V_{0, q}\right\rangle & =\frac{1}{Z} \lim _{z \rightarrow 1} \int d \sigma \frac{e^{2 i \pi\left(\xi_{1}-\xi_{2}\right) \sigma}}{\operatorname{ch}\left(\sigma-m_{2}\right) \operatorname{ch}\left(\sigma-m_{1}\right)} e^{2 \pi q \xi_{2}}=e^{2 \pi q \xi_{2}} \frac{Z}{Z}=e^{2 \pi q \xi_{2}} \\
\left\langle V_{1, q}\right\rangle & =\frac{1}{Z} \lim _{z \rightarrow 1} \int d \sigma \frac{e^{2 i \pi\left(\xi_{1}-\xi_{2}\right) \sigma}}{\operatorname{ch}\left(\sigma-m_{2}\right) \operatorname{ch}\left(\sigma-m_{1}+i q z\right)} e^{2 \pi q \xi_{2}}=e^{2 \pi q \xi_{2}} \frac{\widehat{\mathfrak{S}}^{(q, 0)} Z}{Z} \\
& =(-1)^{q} \frac{e^{2 \pi q \xi_{1}} e^{2 i \pi\left(\xi_{1} m_{1}+\xi_{2} m_{2}\right)}-e^{2 \pi q \xi_{2}} e^{2 i \pi\left(\xi_{1} m_{2}+\xi_{2} m_{1}\right)}}{e^{2 i \pi\left(\xi_{1} m_{1}+\xi_{2} m_{2}\right)}-e^{2 i \pi\left(\xi_{1} m_{2}+\xi_{2} m_{1}\right)}} \\
\left\langle V_{2, q}\right\rangle & =\frac{1}{Z} \lim _{z \rightarrow 1} \int d \sigma \frac{e^{2 i \pi\left(\xi_{1}-\xi_{2}\right) \sigma}}{\operatorname{ch}\left(\sigma-m_{2}+i q z\right) \operatorname{ch}\left(\sigma-m_{1}+i q z\right)} e^{2 \pi q \xi_{2}}=e^{2 \pi q \xi_{2}} \frac{\widehat{\mathfrak{S}}^{(q, q)} Z}{Z}=e^{2 \pi q \xi_{1}}
\end{aligned}
$$

\footnotetext{
${ }^{69}$ As explained before, divergent Wilson loops are computed by deforming the contour of integration.

${ }^{70}$ In section 4.1 we denoted for simplicity $V_{q} \equiv V_{1, q}$.
} 
The mirror symmetry prediction (4.1) is recovered by our exact computations, namely

$$
\left\langle W_{q}\right\rangle \stackrel{\text { mirror }}{\longleftrightarrow}\left\langle V_{1, q}\right\rangle
$$

where $\stackrel{\text { mirror }}{\longleftrightarrow}$ means that the operator vevs are equal under the exchange of masses $m_{j}$ and FI parameters $\xi_{j}$, as required by mirror symmetry.

The explicit results also show that the other Vortex loops $V_{0, q}, V_{2, q}$ are mapped to the flavor Wilson loops of the mirror theory, as predicted in section 4.3.

\subsubsection{Wilson loops in $T[\mathrm{SU}(N)]$}

We turn now to the non-abelian theories by considering Wilson loops in the $T[\mathrm{SU}(N)]$ theories. We pick a Wilson loop in an arbirary representation $\mathcal{R}$ of a $\mathrm{U}(p)$ node, with $1 \leq p \leq N-1$. The matrix model computing it decomposes into a sum of contributions labeled by the weights $w$ of $\mathcal{R}$

$$
\left\langle W_{\mathcal{R}}^{\mathrm{U}(p)}\right\rangle=\sum_{w \in \mathcal{R}} W^{\mathrm{U}(p)}(w)
$$

We compute now the matrix model $W^{\mathrm{U}(p)}(w)$ associated to a single weight $w=$ $\left(w_{1}, w_{2}, \ldots, w_{p}\right)$. To simplify expressions we omit the factors depending on (and the integrals over) the eigenvalues $\sigma_{j}^{(a)}$ with $a>p$ in the matrix model because they do not affect the computation. With this simplification the matrix model is given by

$$
W^{\mathrm{U}(p)}(w)=\frac{1}{Z} \int \prod_{a}^{p}\left[\frac{d^{a} \sigma^{(a)}}{a !} e^{2 \pi \sum_{j}^{p} w_{j} \sigma_{j}^{(p)}} \frac{e^{2 i \pi\left(\xi_{a}-\xi_{a+1}\right) \sum_{j}^{a} \sigma_{j}^{(a)}} \prod_{j<k}^{a} \operatorname{sh}\left(\sigma_{j}^{(a)}-\sigma_{k}^{(a)}\right)^{2}}{\prod_{j, k}^{a, a+1} \operatorname{ch}\left(\sigma_{j}^{(a)}-\sigma_{k}^{(a+1)}\right)}\right] .
$$

The integration over the the eigenvalues $\sigma_{j}^{(a)}$ with $a=1, \ldots, p-1$ just reproduces the partition function of $Z^{T[\mathrm{SU}(p)]}$ with FI parameters $\xi^{(p)}=\left(\xi_{1}, \xi_{2}, \cdots, \xi_{p}\right)$ and mass parameters $\sigma^{(p)}=\left(\sigma_{1}^{(p)}, \sigma_{2}^{(p)}, \cdots, \sigma_{p}^{(p)}\right)$. We can use the formula (5.51) to integrate them out (and relabeling $\left.\sigma_{j}^{(p)}=\sigma_{j}\right)$

$$
\begin{aligned}
& W^{\mathrm{U}(p)}(w) \\
& =\frac{1}{Z} \int \frac{d^{p} \sigma^{(p)}}{p !} Z^{T[\mathrm{SU}(p)]}\left[\xi^{(p)}, \sigma^{(p)}\right] e^{2 \pi \sum_{j}^{p} w_{j} \sigma_{j}^{(p)}} \frac{e^{2 i \pi\left(\xi_{p}-\xi_{p+1}\right) \sum_{j}^{p} \sigma_{j}^{(p)}} \prod_{j<k}^{p} \operatorname{sh}\left(\sigma_{j}^{(p)}-\sigma_{k}^{(p)}\right)^{2}}{\prod_{j, k}^{p, p+1} \operatorname{ch}\left(\sigma_{j}^{(p)}-\sigma_{k}^{(p+1)}\right)} \\
& =\frac{1}{Z} \sum_{\tau \in S_{p}} \int \frac{d^{p} \sigma}{p !} \frac{(-1)^{\tau}(-i)^{\frac{p(p-1)}{2}} e^{2 i \pi \sum_{j}^{p} \xi_{\tau(j)} \sigma_{j}}}{\prod_{j<k}^{p} \operatorname{sh}\left(\xi_{j}-\xi_{k}\right)} e^{2 \pi \sum_{j}^{p} w_{j} \sigma_{j}} \frac{e^{-2 i \pi \xi_{p+1} \sum_{j}^{p} \sigma_{j}} \prod_{j<k}^{p} \operatorname{sh}\left(\sigma_{j}-\sigma_{k}\right)}{\prod_{j, k}^{p, p+1} \operatorname{ch}\left(\sigma_{j}-\sigma_{k}^{(p+1)}\right)} .
\end{aligned}
$$

Relabeling the eigenvalues as $\sigma_{j} \rightarrow \sigma_{\tau(j)}$ in each integral brings the matrix model to the form

$$
\begin{aligned}
& W^{\mathrm{U}(p)}(w) \\
& =\frac{1}{Z} \frac{1}{p !} \sum_{\tau \in S_{p}} \int d^{p} \sigma \frac{(-i)^{\frac{p(p-1)}{2}} e^{2 i \pi \sum_{j}^{p} \xi_{j} \sigma_{j}}}{\prod_{j<k}^{p} \operatorname{sh}\left(\xi_{j}-\xi_{k}\right)} e^{2 \pi \sum_{j}^{p} w_{\tau(j)} \sigma_{j}} \frac{e^{-2 i \pi \xi_{p+1} \sum_{j}^{p} \sigma_{j}} \prod_{j<k}^{p} \operatorname{sh}\left(\sigma_{j}-\sigma_{k}\right)}{\prod_{j, k}^{p, p+1} \operatorname{ch}\left(\sigma_{j}-\sigma_{k}^{(p+1)}\right)} .
\end{aligned}
$$


For each term in the sum over $S_{p}$ the factor $e^{2 \pi \sum_{j}^{p} w_{\tau(j)} \sigma_{j}}$ can be reabsorbed as a shift of the parameters $\xi_{j}$ by $-i w_{\tau(j)}$, for $j=1, \ldots, p$, at the cost of an extra factor $(-1)^{(p-1)\left(w_{1}+w_{2}+\ldots+w_{p}\right)}$. The matrix model without these imaginary shifts is exactly $Z$ (this can be seen by noticing that $Z$ is the matrix model obtained when $w=0$ ). The evaluation of the integrals (with deformed integration contours as explained after (5.36)) then coincides with an analytical continuation of $Z$ to complex FI parameters. We obtain

$$
W^{\mathrm{U}(p)}(w)=\frac{(-1)^{(p-1) w_{\mathrm{tot}}}}{p !} \frac{1}{Z} \sum_{\tau \in S_{p}} \mathfrak{S}^{w_{\tau}} Z
$$

with $w_{\text {tot }}=\sum_{j=1}^{p} w_{j}$ and $w_{\tau}=(w_{\tau(1)}, w_{\tau(2)}, \cdots, w_{\tau(p)}, \underbrace{0, \cdots, 0}_{N-p})$. We need to sum over these single weight contributions to get the final result (5.62). Recognizing $S_{p}$ as the Weyl group $\mathcal{W}$ of $\mathrm{U}(p)$, we can simplify the result using the property

$$
\sum_{w \in \mathcal{R}}\left(\frac{1}{p !} \sum_{\tau \in \mathcal{S}^{p}} F\left[w_{\tau}\right]\right)=\sum_{w \in \mathcal{R}}\left(\frac{1}{|\mathcal{W}|} \sum_{\tau \in \mathcal{W}} F[\tau . w]\right)=\sum_{w \in \mathcal{R}} F[w]
$$

This leads to our final result

$$
\left\langle W_{\mathcal{R}}^{\mathrm{U}(p)}\right\rangle=(-1)^{(p-1)|\mathcal{R}|} \frac{1}{\mathcal{Z}} \sum_{w \in \mathcal{R}} \mathfrak{S}^{\left(w_{1}, w_{2}, \cdots, w_{p}, 0, \cdots, 0\right)} \mathcal{Z},
$$

where we also used the property that for any weight $w, \sum_{j}^{p} w_{j}=|\mathcal{R}|$, the number of boxes in the Young tableau of $\mathcal{R}$, and we replaced trivially $Z$ by $\mathcal{Z}$ defined in (5.52).

\subsubsection{Vortex loops in $T[\mathrm{SU}(N)]$}

We now evaluate the matrix models computing the exact expectation value of Vortex loops in $T[\mathrm{SU}(N)]$. The Vortex loops are labeled by a representation $\mathcal{R}$ of a $\mathrm{U}(p)$ node and, for the $\mathrm{U}(N-1)$ node, by a splitting $N=M+(N-M)$ of the fundamental hypermultiplets (see section 4.3). We denote by $V_{\mathcal{R}}^{(p)}, 1 \leq p \leq N-2$, the $\mathrm{U}(p)$ Vortex loops and by $V_{M, \mathcal{R}}$ the $\mathrm{U}(N-1)$ Vortex loops. As for the Wilson loops, the matrix model computing a Vortex loop $V_{\mathcal{R}}$, which is labeled (in particular) by a representation $\mathcal{R}$, decomposes into a sum of contributions labeled by the weights $w$ of $\mathcal{R}$

$$
\left\langle V_{\mathcal{R}}\right\rangle=\sum_{w \in \mathcal{R}} V(w) .
$$

This decomposition in a sum over weights follows from the evaluation of the SQM index (5.45), (5.47). We will be computing the matrix model $V(w)$ associated to a single weight $w=\left(w_{1}, w_{2}, \ldots, w_{p}\right)$ for each Vortex loop.

Let us first discuss Vortex loops in the $\mathrm{U}(p)$ nodes with $1 \leq p \leq N-2$. The prediction from the brane picture of section 4.3 is that such a Vortex loop must evaluate to a background Wilson loop for a global symmetry, depending on the parameters $\xi_{j}, 1 \leq j \leq p$. In the special case of the $T[\mathrm{SU}(N)]$ theory, the brane picture predicts that the Vortex loop $V_{\mathcal{R}}^{(p)}$ will evaluate to a background Wilson loop transforming in a representation $\mathcal{R}$ of 
the topological symmetry subgroup $\mathrm{U}(p) \subset \mathrm{U}(N)_{J}=G_{C}$ acting on the Coulomb branch, associated to the deformation parameters $\xi_{j}, 1 \leq j \leq p$.

To compute the vev of this Vortex loop, we choose to consider its left-SQM quiver realization. The matrix model is given by (5.48) with the defect contribution (5.47) and additional background loop (5.49). We simplify the matrix model by replacing the factors depending on the eigenvalues $\sigma_{j}^{(a)}$ with $a \geq p$, which do not affect the computation, by $\int[\cdots]$. This gives for the contribution of a single weight $w=\left(w_{1}, w_{2}, \cdots, w_{p}\right)$ to the Vortex loop

$$
\begin{aligned}
V^{(p)}(w)= & \lim _{z \rightarrow 1} \frac{1}{Z} \int[\cdots] \int \prod_{a}^{p-1}\left[\frac{d^{a} \sigma^{(a)}}{a !} \frac{e^{2 i \pi\left(\xi_{a}-\xi_{a+1}\right) \sum_{j}^{a} \sigma_{j}^{(a)}} \prod_{j<k}^{a} \operatorname{sh}\left(\sigma_{j}^{(a)}-\sigma_{k}^{(a)}\right)^{2}}{\prod_{j, k}^{a, a+1} \operatorname{ch}\left(\sigma_{j}^{(a)}-\sigma_{k}^{(a+1)}\right)}\right] \\
& \times e^{2 \pi w_{\text {tot }} \xi_{p}} \frac{\prod_{j, k}^{p-1, p} \operatorname{ch}\left(\sigma_{j}^{(p-1)}-\sigma_{k}^{(p)}\right)}{\prod_{j, k}^{p-1, p} \operatorname{ch}\left(\sigma_{j}^{(p-1)}-\sigma_{k}^{(p)}+i w_{k} z\right)} \\
= & \lim _{z \rightarrow 1} \frac{1}{Z} \int[\cdots] \int \prod_{a}^{p-1} \frac{d^{a} \sigma^{(a)}}{a !} \prod_{a}^{p-2}\left[\frac{d^{a} \sigma^{(a)}}{a !} \frac{e^{2 i \pi\left(\xi_{a}-\xi_{a+1}\right) \sum_{j}^{a} \sigma_{j}^{(a)}} \prod_{j<k}^{a} \operatorname{sh}\left(\sigma_{j}^{(a)}-\sigma_{k}^{(a)}\right)^{2}}{\prod_{j, k}^{a, a+1} \operatorname{ch}\left(\sigma_{j}^{(a)}-\sigma_{k}^{(a+1)}\right)}\right] \\
& \times e^{2 \pi w_{\text {tot }} \xi_{p}} \frac{e^{2 i \pi\left(\xi_{p-1}-\xi_{p}\right) \sum_{j}^{p-1} \sigma_{j}^{(p-1)}} \prod_{j<k}^{p-1} \operatorname{sh}\left(\sigma_{j}^{(p-1)}-\sigma_{k}^{(p-1)}\right)^{2}}{\prod_{j, k}^{p-1, p} \operatorname{ch}\left(\sigma_{j}^{(p-1)}-\sigma_{k}^{(p)}+i w_{k} z\right)}
\end{aligned}
$$

where $w_{\text {tot }}=\sum_{k=1}^{p} w_{k}=|\mathcal{R}|$. We remind that $\lim _{z \rightarrow 1}$ means that we compute the matrix model for $z \in i \mathbb{R}$ and analytically continue the result to $z=1$. For $z \in i \mathbb{R}$, we recognize in the integrand the matrix model computing the partition function of $T[\mathrm{SU}(p)]$ with FI parameters $\xi^{(p)}=\left(\xi_{1}, \xi_{2}, \cdots, \xi_{p}\right)$ and shifted mass parameters $\sigma^{(p)}-i w z=\left(\sigma_{1}^{(p)}-\right.$ $\left.i w_{1} z, \sigma_{2}^{(p)}-i w_{2} z, \cdots, \sigma_{p}^{(p)}-i w_{p} z\right)$. We can use (5.51) to evaluate it and easily perform the analytic continuation to $z=1,{ }^{71}$

$$
\begin{aligned}
& V^{(p)}(w) \\
& =\lim _{z \rightarrow 1} \frac{1}{Z} \int[\cdots] e^{2 \pi w_{\mathrm{tot}} \xi_{p}} Z^{T[\operatorname{SU}(p)]}\left[\xi^{(p)}, \sigma^{(p)}-i w z\right] \\
& =\frac{1}{Z} \int[\cdots](-i)^{\frac{p(p-1)}{2}} e^{-2 i \pi \xi_{p} \sum_{j} \sigma_{j}^{(p)}}(-1)^{(p-1) w_{\mathrm{tot}}} \frac{\sum_{\tau \in S_{p}}(-1)^{\tau} e^{2 \pi \sum_{j}^{p} \xi_{j} w_{\tau(j)}} e^{2 \pi i \sum_{j}^{p} \xi_{j} \sigma_{\tau(j)}^{(p)}}}{\prod_{j<k}^{p} \operatorname{sh}\left(\xi_{j}-\xi_{k}\right) \operatorname{sh}\left(\sigma_{j}^{(p)}-\sigma_{k}^{(p)}\right)} .
\end{aligned}
$$

Note that the background factor $e^{2 \pi w_{\text {tot }} \xi_{p}}$ canceled in the limit $z=1$. One can now pull out the sum over permutations $\tau \in S_{p}$ and play with eigenvalues relabelings $\sigma_{\tau(j)}^{(p)} \leftrightarrow \sigma_{j}^{(p)}$ (knowing that the hidden factors in $[\cdots]$ are invariant under such relabelings). After several

\footnotetext{
${ }^{71}$ The analytic continuation $z \rightarrow 1$ can be directly taken in the integrand for the factor $\prod_{j<k}^{p} \operatorname{sh}^{-1}\left(\sigma_{j}^{(p)}-\right.$ $\left.\sigma_{k}^{(p)}-i w_{j} z+i w_{k} z\right)$, as no pole crosses the contour of integration when $z \rightarrow 1$. This is because the rest of the matrix integrand has $\prod_{j<k}^{p} \operatorname{sh}\left(\sigma_{j}^{(p)}-\sigma_{k}^{(p)}\right)$ factors, which kill the poles of the first factor. We will rely on this property on several other occasions. Enforcing $z=1$ simplifies the factor to $(-1)^{(p-1) w_{\text {tot }}} \prod_{j<k}^{p} \operatorname{sh}^{-1}\left(\sigma_{j}^{(p)}-\sigma_{k}^{(p)}\right)$.
} 
operations we can pull out of the integral the factors depending on the weight $w$,

$$
\begin{aligned}
V^{(p)}(w)= & (-1)^{(p-1) w_{\text {tot }}} \frac{1}{p !} \sum_{\tau \in S_{p}} e^{2 \pi \sum_{j}^{p} \xi_{j} w_{\tau(j)}} \\
& \times \frac{1}{Z} \int[\cdots](-i)^{\frac{p(p-1)}{2}} e^{-2 i \pi \xi_{p} \sum_{j} \sigma_{j}^{(p)}} \frac{\sum_{\tau \in S_{p}}(-1)^{\tau} e^{2 \pi i \sum_{j}^{p} \xi_{j} \sigma_{\tau(j)}^{(p)}}}{\prod_{j<k}^{p} \operatorname{sh}\left(\xi_{j}-\xi_{k}\right) \operatorname{sh}\left(\sigma_{j}^{(p)}-\sigma_{k}^{(p)}\right)} \\
= & (-1)^{(p-1) w_{\text {tot }}} \frac{1}{p !} \sum_{\tau \in S_{p}} e^{2 \pi \sum_{j}^{p} \xi_{j} w_{\tau(j)}} \frac{1}{Z} \int[\cdots] Z^{T[\mathrm{SU}(p)]}\left[\xi^{(p)}, \sigma^{(p)}\right] .
\end{aligned}
$$

In the last step we have re-transformed the matrix model integrand into the $Z^{T[\mathrm{SU}(p)]}$ partition function, but with unshifted mass parameters. The resulting matrix model is simply the bare $Z^{T[\mathrm{SU}(N)]}$ matrix model, which cancels with the normalization $1 / Z$, leaving only the $w$ dependent prefactor

$$
V^{(p)}(w)=(-1)^{(p-1) w_{\text {tot }}} \frac{1}{p !} \sum_{\tau \in S_{p}} e^{2 \pi \sum_{j}^{p} \xi_{j} w_{\tau(j)}} .
$$

The full Vortex loop vev is obtained by summing over all the single weight contributions. Using (5.67) we obtain

$$
\left\langle V_{\mathcal{R}}^{(p)}\right\rangle=(-1)^{(p-1)|\mathcal{R}|} \sum_{w \in \mathcal{R}} e^{2 \pi \sum_{j}^{p} \xi_{j} w_{j}}
$$

where $|\mathcal{R}|\left(=w_{\text {tot }}\right)$ is the total number of boxes in the Young tableau associated to the representation $\mathcal{R}$. Up to the overall sign factor, $V_{\mathcal{R}}^{(p)}$ evaluates to a background Wilson loop in the representation $\mathcal{R}$ of the $\mathrm{U}(p) \subset \mathrm{U}(N)_{J}$ subgroup of the topological symmetry acting on the Coulomb branch. This is precisely the prediction (4.7) derived from the brane picture.

We now turn to the evaluation of the vevs of the Vortex loops $V_{M, \mathcal{R}}$ of the $\mathrm{U}(N-1)$ node.

Note that the Vortex loop vev $\left\langle V_{N, \mathcal{R}}\right\rangle$, associated to the splitting of hypermultiplets $N=N+0$, can be evaluated in exactly the same fashion as the vevs of the $V_{\mathcal{R}}^{(p)}$ loops, leading to the result (5.74) with $p=N-1$ and reproduces our prediction (4.8).

In order to evaluate the $V_{M, \mathcal{R}}$ vevs, we are going to consider their "right" SQM realization, namely we choose to insert in the $3 \mathrm{~d}$ matrix model the right SQM index (5.45) with the additional background loop (5.49). Again we start by considering a single weight contribution $V_{M}(w)$, with $w=\left(w_{1}, \cdots, w_{N-1}\right)$, and we simplify the matrix model by replacing the factors irrelevant to the computation by $\int[\cdots]$,

$$
\begin{aligned}
V_{M}(w)= & \lim _{z \rightarrow 1} \frac{1}{Z} \int[\cdots] \int \frac{d^{N^{\prime}} \sigma}{N^{\prime} !} \frac{\prod_{i<j}^{N^{\prime}} \operatorname{sh}^{2}\left(\sigma_{i}-\sigma_{j}\right)}{\prod_{k=1}^{N} \prod_{j}^{N^{\prime}} \operatorname{ch}\left(\sigma_{j}-m_{k}\right)} \\
& \times e^{2 \pi \xi_{N} w_{\text {tot }}} \frac{\prod_{k=1}^{M} \prod_{j}^{N^{\prime}} \operatorname{ch}\left(m_{k}-\sigma_{j}\right)}{\prod_{k=1}^{M} \prod_{j}^{N^{\prime}} \operatorname{ch}\left(m_{k}-\sigma_{j}-i w_{j} z\right)},
\end{aligned}
$$


where $\sigma_{j}$ denote the eigenvalues of the $\mathrm{U}(N-1)$ node and we defined $N^{\prime} \equiv N-1$ for convenience. The cancellation between numerator and denominator factors leads to

$$
V_{M}(w)=\lim _{z \rightarrow 1} \frac{1}{Z} \int[\cdots] \int \frac{d^{N^{\prime}} \sigma}{N^{\prime} !} \frac{e^{2 \pi \xi_{N} w_{\text {tot }}} \prod_{i<j}^{N^{\prime}} \operatorname{sh}^{2}\left(\sigma_{i}-\sigma_{j}\right)}{\prod_{k=M+1}^{N} \prod_{j}^{N^{\prime}} \operatorname{ch}\left(\sigma_{j}-m_{k}\right) \prod_{k=1}^{M} \prod_{j}^{N^{\prime}} \operatorname{ch}\left(\sigma_{j}+i w_{j} z-m_{k}\right)} .
$$

We compute this matrix model by using a generalized Cauchy determinant formula. Let us remind the Cauchy determinant formula

$$
\frac{\prod_{i<j}^{N} \operatorname{sh}\left(\sigma_{i}-\sigma_{j}\right) \prod_{i<j}^{N} \operatorname{sh}\left(\widetilde{\sigma}_{i}-\widetilde{\sigma}_{j}\right)}{\prod_{i, j}^{N} \operatorname{ch}\left(\sigma_{i}-\widetilde{\sigma}_{j}\right)}=\sum_{\tau \in S_{N}}(-1)^{\tau} \frac{1}{\prod_{j}^{N} \operatorname{ch}\left(\sigma_{\tau(j)}-\widetilde{\sigma}_{j}\right)} .
$$

A generalized version of this formula, for $N \geq \tilde{N}, \Delta \equiv N-\tilde{N}$, is

$$
\begin{aligned}
& \frac{\prod_{i<j}^{N} \operatorname{sh}\left(\sigma_{i}-\sigma_{j}\right) \prod_{i<j}^{\widetilde{N}} \operatorname{sh}\left(\widetilde{\sigma}_{i}-\widetilde{\sigma}_{j}\right)}{\prod_{i}^{N} \prod_{j}^{\widetilde{N}} \operatorname{ch}\left(\sigma_{i}-\widetilde{\sigma}_{j}\right)} \\
& \quad=(-1)^{\Delta \widetilde{N}} \sum_{\tau \in S_{N}}(-1)^{\tau} \prod_{j=1}^{\widetilde{N}} \frac{e^{-\pi \Delta\left(\sigma_{\tau(j)}-\widetilde{\sigma}_{j}\right)}}{\operatorname{ch}\left(\sigma_{\tau(j)}-\widetilde{\sigma}_{j}\right)} \prod_{j=\widetilde{N}+1}^{N} e^{2 \pi \sigma_{\tau(j)}\left(\frac{N+\widetilde{N}+1}{2}-j\right)} .
\end{aligned}
$$

We use this formula in the matrix model to replace the factor depending on the weight $w$, with $\Delta=N^{\prime}-M$,

$$
\begin{aligned}
\frac{1}{\prod_{k=1}^{M} \prod_{j=1}^{N^{\prime}} \operatorname{ch}\left(\sigma_{j}+i w_{j} z-m_{k}\right)} \\
=\frac{(-1)^{\Delta M}}{\prod_{i<j}^{N^{\prime}} \operatorname{sh}\left(\sigma_{i}-\sigma_{j}+i\left(w_{i}-w_{j}\right) z\right) \prod_{i<j}^{M} \operatorname{sh}\left(m_{i}-m_{j}\right)} \\
\quad \times \sum_{\tau \in S_{N^{\prime}}}(-1)^{\tau} \prod_{j=1}^{M} \frac{e^{-\pi \Delta\left(\sigma_{\tau(j)}+i w_{\tau(j)} z-m_{j}\right)}}{\operatorname{ch}\left(\sigma_{\tau(j)}+i w_{\tau(j)} z-m_{j}\right)} \prod_{j=M+1}^{N^{\prime}} e^{2 \pi\left(\sigma_{\tau(j)}+i w_{\tau(j)} z\right)\left(\frac{N^{\prime}+M+1}{2}-j\right)} .
\end{aligned}
$$

We may now plug this result into the matrix model and pull out the sum over permutation $\tau \in S_{N^{\prime}}$

$$
\begin{aligned}
& V_{M}(w) \\
& =\lim _{z \rightarrow 1} \sum_{\tau \in S_{N^{\prime}}} \frac{(-1)^{\tau}}{Z} \int[\cdots] \int \frac{d^{N^{\prime}} \sigma}{N^{\prime} !} \frac{\prod_{i<j}^{N^{\prime}} \operatorname{sh}^{2}\left(\sigma_{i}-\sigma_{j}\right) \prod_{j=M+1}^{N^{\prime}} e^{2 \pi i w_{\tau(j)} z\left(\frac{N^{\prime}+M+1}{2}-j\right)}}{\prod_{k=M+1}^{N} \prod_{j}^{N^{\prime}} \operatorname{ch}\left(\sigma_{j}-m_{k}\right) \prod_{i<j}^{N^{\prime}} \operatorname{sh}\left(\sigma_{i}-\sigma_{j}+i\left(w_{i}-w_{j}\right) z\right)} \\
& \quad \times \frac{(-1)^{\Delta M} e^{2 \pi \xi_{N} w_{\text {tot }}}}{\prod_{i<j}^{M} \operatorname{sh}\left(m_{i}-m_{j}\right)} \prod_{j=1}^{M} \frac{e^{-\pi \Delta\left(\sigma_{\tau(j)}+i w_{\tau(j)} z-m_{j}\right)}}{\operatorname{ch}\left(\sigma_{\tau(j)}+i w_{\tau(j)} z-m_{j}\right)} \prod_{j=M+1}^{N^{\prime}} e^{2 \pi \sigma_{\tau(j)}\left(\frac{N^{\prime}+M+1}{2}-j\right)}
\end{aligned}
$$

We can simplify the result by taking directly setting $z=1$ in the factors in the first line of (5.79), which just produces a sign. This is justified because these factors do not have 
poles crossing the integration contours as $z$ goes from 0 to 1 . We obtain

$$
\begin{aligned}
& V_{M}(w) \\
& =\lim _{z \rightarrow 1} \sum_{\tau \in S_{N^{\prime}}} \frac{(-1)^{\tau}}{Z} \int[\cdots] \int \frac{d^{N^{\prime}} \sigma}{N^{\prime} !} \frac{(-1)^{N w_{\text {tot }}}(-1)^{(N+M) \sum_{j=M+1}^{N^{\prime}} w_{\tau(j)}} \prod_{i<j}^{N^{\prime}} \operatorname{sh}\left(\sigma_{i}-\sigma_{j}\right)}{\prod_{k=M+1}^{N} \prod_{j}^{N^{\prime}} \operatorname{ch}\left(\sigma_{j}-m_{k}\right)} \\
& \quad \times \frac{(-1)^{\Delta M} e^{2 \pi \xi_{N} w_{\text {tot }}}}{\prod_{i<j}^{M} \operatorname{sh}\left(m_{i}-m_{j}\right)} \prod_{j=1}^{M} \frac{e^{-\pi \Delta\left(\sigma_{\tau(j)}+i w_{\tau(j)} z-m_{j}\right)}}{\operatorname{ch}\left(\sigma_{\tau(j)}+i w_{\tau(j)} z-m_{j}\right)} \prod_{j=M+1}^{N^{\prime}} e^{2 \pi \sigma_{\tau(j)}\left(\frac{N^{\prime}+M+1}{2}-j\right)}
\end{aligned}
$$

It is now possible to relabel the eigenvalues $\sigma_{\tau(j)} \leftrightarrow \sigma_{j}$ in each integral and recognize each term in the sum as the same matrix model with shifted masses $m_{j} \rightarrow m_{j}-i w_{\tau(j)} z$, for $1 \leq j \leq p$. The shifted matrix model is simply the bare partition function $Z$, as can be understood by taking $w_{j}=0$ for all $j$. We have precisely

$$
\begin{aligned}
V_{M}(w)= & \lim _{z \rightarrow 1} \frac{1}{N^{\prime} !} \sum_{\tau \in S_{N^{\prime}}} \frac{1}{Z} \int[\cdots] \int d^{N^{\prime}} \sigma \frac{(-1)^{N w_{\text {tot }}}(-1)^{(N+M) \sum_{j=M+1}^{N^{\prime}} w_{\tau(j)}} \prod_{i<j}^{N^{\prime}} \operatorname{sh}\left(\sigma_{i}-\sigma_{j}\right)}{\prod_{k=M+1}^{N} \prod_{j}^{N^{\prime}} \operatorname{ch}\left(\sigma_{j}-m_{k}\right)} \\
& \times \frac{(-1)^{\Delta M} e^{2 \pi \xi_{N} w_{\text {tot }}}}{\prod_{i<j}^{M} \operatorname{sh}\left(m_{i}-m_{j}\right)} \prod_{j=1}^{M} \frac{e^{-\pi \Delta\left(\sigma_{j}+i w_{\tau(j)} z-m_{j}\right)}}{\operatorname{ch}\left(\sigma_{j}+i w_{\tau(j)} z-m_{j}\right)} \prod_{j=M+1}^{N^{\prime}} e^{2 \pi \sigma_{j}\left(\frac{N^{\prime}+M+1}{2}-j\right)} \\
= & e^{2 \pi \xi_{N} w_{\text {tot }}}(-1)^{M w_{\text {tot }}} \frac{1}{N^{\prime} !} \sum_{\tau \in S_{N^{\prime}}}(-1)^{N^{\prime} \sum_{j=1}^{M} w_{\tau(j)}} \frac{1}{Z} \widehat{\mathfrak{S}}^{w_{M, \tau}} Z
\end{aligned}
$$

where $w_{M, \tau}=(w_{\tau(1)}, w_{\tau(2)}, \cdots, w_{\tau(M)}, \underbrace{0, \cdots, 0}_{N-M})$. Summing over the weights $w$ of the representation $\mathcal{R}$ and using (5.67) yields the final result

$$
\left\langle V_{M, \mathcal{R}}\right\rangle=e^{2 \pi \xi_{N} w_{\text {tot }}}(-1)^{M|\mathcal{R}|} \sum_{w \in \mathcal{R}}(-1)^{(N-1) \sum_{j=1}^{M} w_{j}} \frac{1}{Z} \widehat{\mathfrak{S}}^{w_{M}} Z,
$$

where $w_{M}=(w_{1}, w_{2}, \cdots, w_{M}, \underbrace{0, \cdots, 0}_{N-M})$. To check mirror symmetry, it is useful to reexpress the result in terms of $\mathcal{Z}$, which is manifestly invariant under the exchange of mass and FI parameters,

$$
\left\langle V_{M, \mathcal{R}}\right\rangle=(-1)^{(N+M-1)|\mathcal{R}|} \sum_{w \in \mathcal{R}} e^{2 \pi \check{\xi}_{N} \sum_{j=M+1}^{N-1} w_{j}} \frac{1}{\mathcal{Z}} \widehat{\mathfrak{S}}^{w_{M}} \mathcal{Z},
$$

where we have reabsorbed $(-1)$ factors into an imaginary shift of $\xi_{N}$ with $\check{\xi}_{N}=\xi_{N}+i \frac{N-1}{2}$. We remind the reader that we did not keep track of the overall sign in the evaluation of the SQM index, so that our evaluation of Vortex loops are only valid up to an overall sign.

This completes our evaluation of the matrix models computing the vevs of the $T[\mathrm{SU}(N)]$ Vortex loop operators.

\subsubsection{Mirror map}

With all the computations out of the way, it remains to confirm the rest of the mirror map predictions of section 4 between loop operators in $T[\mathrm{SU}(N)]$. Let start with the simplest 
case of the Vortex loop $V_{N-1, \mathcal{R}}$ discussed in section 4.2. Its vev is given by (5.83) with $M=N-1$,

$$
\left\langle V_{N-1, \mathcal{R}}\right\rangle=\frac{1}{\mathcal{Z}} \sum_{w \in \mathcal{R}} \widehat{\mathfrak{S}}^{w} \mathcal{Z}
$$

where we used $\sum_{j=1}^{N-1} w_{j}=w_{\text {tot }}=|\mathcal{R}|$. Recalling the vev of the Wilson loop in the $\mathrm{U}(N-1)$ node given by (5.68),

$$
\left\langle W_{\mathcal{R}}^{\mathrm{U}(N-1)}\right\rangle=(-1)^{N|\mathcal{R}|} \frac{1}{\mathcal{Z}} \sum_{w \in \mathcal{R}} \mathfrak{S}^{w} \mathcal{Z}
$$

we observe that the two vevs are mapped, up to an irrelevant sign, under the exchange of the masses and FI parameters $m_{j} \leftrightarrow \xi_{j}$,

$$
\left\langle W_{\mathcal{R}}^{\mathrm{U}(N-1)}\right\rangle \stackrel{\text { mirror }}{\longleftrightarrow}\left\langle V_{N-1, \mathcal{R}}\right\rangle .
$$

This is precisely the prediction (4.4) of mirror symmetry obtained from the brane picture.

The more complicated mirror symmetry predictions of section 4.3 are also easily checked with our explicit exact results. The formula (5.83) for the $V_{M, \mathcal{R}}$ loops vevs expresses a decomposition into contributions labeled by representations $(\mathbf{q}, \widehat{\mathcal{R}})$ appearing in the decomposition of $\mathcal{R}$ under the subgroup $\mathrm{U}(1) \times \mathrm{U}(M)$, where $\mathrm{U}(M)$ is embedded as $\mathrm{U}(M) \times \mathrm{U}(N-M-1) \subset \mathrm{U}(N-1)$ and $\mathrm{U}(1)$ is embedded diagonally in $\mathrm{U}(N-M-1)$,

$$
\mathrm{U}(N-1) \rightarrow \mathrm{U}(1) \times \mathrm{U}(M): \quad \mathcal{R} \rightarrow \bigoplus_{s \in \Delta_{M}}\left(\mathbf{q}_{s}, \widehat{\mathcal{R}}_{s}\right)
$$

where $\Delta_{M}$ denotes the set of representations $(\mathbf{q}, \widehat{\mathcal{R}})$ in the decomposition of $\mathcal{R}$ counted with degeneracies. Equation (5.83) can be re-expressed as

$$
\left\langle V_{M, \mathcal{R}}\right\rangle=(-1)^{M|\mathcal{R}|} \sum_{s \in \Delta_{M}}\left[e^{2 \pi \mathbf{q}_{s} \check{\xi}_{N}} \frac{1}{\mathcal{Z}} \sum_{\widehat{w} \in \widehat{\mathcal{R}}_{s}} \widehat{\mathfrak{S}}^{\widehat{w}} \mathcal{Z}\right] .
$$

The factor $\frac{1}{\mathcal{Z}} \sum_{\widehat{w} \in \widehat{\mathcal{R}}_{s}} \widehat{\mathfrak{S}}^{\widehat{w}} \mathcal{Z}$ is labeled by a representation $\widehat{\mathcal{R}}_{s}$ of $\mathrm{U}(M)$ and is mapped under the exchange of mass and FI parameters to the vev given in (5.68) of the $\mathrm{U}(M)$ Wilson loop labeled by a representation $\widehat{\mathcal{R}}_{s}$. We obtain the explicit mirror symmetry map

$$
\left\langle V_{M, \mathcal{R}}\right\rangle \stackrel{\text { mirror }}{\longleftrightarrow}(-1)^{N|\mathcal{R}|} \sum_{s \in \Delta_{M}} e^{2 \pi \mathbf{q}_{s} \check{\tilde{m}}_{N}}\left\langle W_{\widehat{\mathcal{R}}_{s}}^{\mathrm{U}(M)}\right\rangle
$$

where we have used $N_{\widehat{\mathcal{R}}_{s}}+\mathbf{q}_{s}=|\mathcal{R}|$ and $\check{\widetilde{m}}_{N}=\xi_{N}+i \frac{N-1}{2}$ is a fundamental hypermultiplet mass in the mirror dual $T[\mathrm{SU}(N)]$, shifted by an imaginary number to reabsorb $(-1)$ factors. This expresses the fact that the $V_{M, \mathcal{R}}$ Vortex loop is mapped under mirror symmetry to a linear combination of $W_{\widehat{\mathcal{R}}}^{\mathrm{U}(M)}$ Wilson loops combined with flavor Wilson loops. This reproduces the mirror symmetry prediction (4.6) found by studying carefully the brane realization of the loop operators and their mapping under S-duality, up to the imaginary shift of $\widetilde{m}_{N}=\xi_{N}$. This imaginary shift of the flavor parameter in the background Wilson loop is curious and would deserve more investigation. 
This completes successfully the checks of mirror symmetry for the $T[\mathrm{SU}(N)]$ theory. We have found that $T[\mathrm{SU}(N)]$ Wilson loops and Vortex loops can be expressed as operators acting on the partition function by imaginary shifts of the FI or mass parameters (see also [50]). More generally, in all $T_{\hat{\rho}}^{\rho}[\mathrm{SU}(N)]$ linear quiver theories it seems possible to express Wilson loops / Vortex loops as operators acting on the partition function by imaginary shifts of a "generalized" set of FI parameters/ mass parameters. (we will not provide a proof of this result in this paper).

\subsection{Loops in SQCD}

As our final example, we consider loops in the $\mathrm{U}(N)$ theory with $2 N$ fundamental and its mirror dual theory, discussed in section 4.5. We focus on the prediction (4.15), relating the Wilson loop $W_{\mathcal{R}}$ of the $\mathrm{U}(N)$ theory to the Vortex loop $\widetilde{V}_{1, \mathcal{R}}^{(N)}$ of the mirror theory. The other mirror maps (4.16) can be easily checked with the explicit matrix models computing the vevs of the operators, by computations essentially identical to those presented above for the $T[\mathrm{SU}(N)]$ loops.

We denote $\xi_{1}-\xi_{2}$ the FI parameter and $m_{a}, a=1, \cdots, 2 N$, the masses of fundamental hypermultiplets in the $\mathrm{U}(N)$ theory, $\widetilde{\xi}_{a}-\widetilde{\xi}_{a+1}, a=1, \cdots, 2 N-1$, the FI parameters and $\widetilde{m}_{1}, \widetilde{m}_{2}$ the masses of fundamental hypermultiplets in the mirror theory. The matrix models computing the $S^{3}$ partition function of the $\mathrm{U}(N)$ theory and its mirror dual are given by

$$
\begin{aligned}
Z= & e^{\phi} \int \frac{d^{N} \sigma}{N !} \frac{\prod_{i<j}^{N} \operatorname{sh}^{2}\left(\sigma_{i}-\sigma_{j}\right)}{\prod_{j}^{N} \prod_{a}^{2 N} \operatorname{ch}\left(\sigma_{j}-m_{a}\right)} e^{2 \pi i\left(\xi_{1}-\xi_{2}\right) \sum_{j}^{N} \sigma_{j}}, \\
\widetilde{Z}= & e^{\widetilde{\phi}} \int \frac{d^{N} \sigma}{N !} \mathcal{Z}^{T[\operatorname{SU}(N)]}\left[\widetilde{\xi}_{1 \ldots N} ; \sigma_{1 \ldots N}\right] \frac{\prod_{i<j}^{N} \operatorname{sh}^{2}\left(\sigma_{i}-\sigma_{j}\right)}{\prod_{j}^{N} \operatorname{ch}\left(\sigma_{j}-\widetilde{m}_{1}\right) \operatorname{ch}\left(\sigma_{j}-\widetilde{m}_{2}\right)} \\
& \times \mathcal{Z}^{T[\mathrm{SU}(N)]}\left[-\widetilde{\xi}_{N+1 \ldots 2 N} ; \sigma_{1 \ldots N}\right],
\end{aligned}
$$

where we used to fact that the mirror theory can be decomposed into three pieces: two $T[\mathrm{SU}(N)]$ theories (left and right parts of the quiver in figure 33-b) whose $\mathrm{SU}(N)$ hypermultiplet flavor symmetries are gauged with the $\mathrm{U}(N)$ gauge symmetry of the central node (which has two more hypermultiplets by itself). The matrix model is then a combination of these three pieces and can be expressed using two $T[\mathrm{SU}(N)]$ partition functions with mass parameters identified with the $\mathrm{U}(N)$ node eigenvalues $\sigma_{j}$ and FI parameters as indicated. We have also added background Chern-Simons terms given by

$$
e^{\phi}=e^{2 \pi i \xi_{2} \sum_{a=1}^{2 N} m_{a}}, \quad e^{\widetilde{\phi}}=e^{2 \pi i\left(\widetilde{m}_{1}+\widetilde{m}_{2}\right) \sum_{a=N+1}^{2 N} \widetilde{\xi}_{a}},
$$

which are unphysical, as they belong to the set of finite counterterms parametrizing ambiguities of the partition function, but are useful to obtain partition functions which match exactly under mirror symmetry, namely under the identification

$$
\left(\xi_{1}, \xi_{2}, m_{a}\right)=\left(\widetilde{m}_{1}, \widetilde{m}_{2}, \widetilde{\xi}_{a}\right)
$$

This result will follow from our computations. 
The matrix model computing the vev of a Wilson loop $W_{\mathcal{R}}$ in the $\mathrm{U}(N)$ theory is given by

$$
\begin{aligned}
\left\langle W_{\mathcal{R}}\right\rangle & =\sum_{w \in \mathcal{R}} W(w) \\
W(w) & =\frac{e^{\phi}}{Z} \int \frac{d^{N} \sigma}{N !} e^{2 \pi \sum_{j}^{N} w_{j} \sigma_{j}} \frac{\prod_{i<j}^{N} \operatorname{sh}^{2}\left(\sigma_{i}-\sigma_{j}\right)}{\prod_{j}^{N} \prod_{a}^{2 N} \operatorname{ch}\left(\sigma_{j}-m_{a}\right)} e^{2 \pi i\left(\xi_{1}-\xi_{2}\right) \sum_{j}^{N} \sigma_{j}}
\end{aligned}
$$

Using twice the Cauchy determinant formula (5.77) we find

$$
W(w)=\sum_{\tau, \tau^{\prime} \in S_{N}}(-1)^{\tau+\tau^{\prime}} \frac{e^{\phi}}{Z} \frac{1}{\prod\left[m_{a}\right]} \int \frac{d^{N} \sigma}{N !} \frac{e^{2 \pi \sum_{j}^{N} w_{j} \sigma_{j}} e^{2 \pi i\left(\xi_{1}-\xi_{2}\right) \sum_{j}^{N} \sigma_{j}}}{\prod_{j}^{N} \operatorname{ch}\left(\sigma_{j}-m_{\tau(j)}\right) \operatorname{ch}\left(\sigma_{j}-m_{\tau^{\prime}(j)+N}\right)},
$$

where we define $\prod\left[x_{a}\right] \equiv \prod_{1 \leq a<b \leq N} \operatorname{sh}\left(x_{a}-x_{b}\right) \operatorname{sh}\left(x_{a+N}-x_{b+N}\right)$. To compute the integrals we use the identity

$$
\int d \sigma \frac{e^{2 \pi i \beta \sigma}}{\operatorname{ch}\left(\sigma-M_{1}\right) \operatorname{ch}\left(\sigma-M_{2}\right)}=(-i) \frac{e^{2 \pi i \beta M_{1}}-e^{2 \pi i \beta M_{2}}}{\operatorname{sh} \beta \operatorname{sh}\left(M_{1}-M_{2}\right)}
$$

analytically continued to $\beta \in \mathbb{C}$. After simplifications we obtain

$$
\begin{aligned}
W(w)= & \sum_{\tau, \tau^{\prime} \in S_{N}} \frac{(-1)^{\tau+\tau^{\prime}}}{Z} \frac{(-i)^{N}(-1)^{|\mathcal{R}|}}{N ! \prod\left[m_{a}\right] \operatorname{sh}^{N}\left(\xi_{1}-\xi_{2}\right)} \\
& \times \prod_{j=1}^{N} \frac{e^{2 \pi i\left(\xi_{1} m_{\tau(j)}+\xi_{2} m_{\tau^{\prime}(j)+N}-i w_{j} m_{\tau(j)}\right)}-e^{2 \pi i\left(\xi_{2} m_{\tau(j)}+\xi_{1} m_{\tau^{\prime}(j)+N}-i w_{j} m_{\tau^{\prime}(j)+N}\right)}}{\operatorname{sh}\left(m_{\tau(j)}-m_{\tau^{\prime}(j)+N}\right)} .
\end{aligned}
$$

We now turn to the mirror dual operator which should be the Vortex loop $\widetilde{V}_{1, \mathcal{R}}^{(N)}$, according to the discussion in section 4.5. We compute its vev from its $3 \mathrm{~d} / 1 \mathrm{~d}$ realization with the left SQM. The matrix model following from (5.48), with SQM factor (5.47) and background loop (5.49) can be expressed after cancellations of factors of ch as

$$
\begin{aligned}
\left\langle\widetilde{V}_{1, \mathcal{R}}^{(N)}\right\rangle= & \sum_{w \in \mathcal{R}} \widetilde{V}(w) \\
\widetilde{V}(w)= & \frac{1}{\widetilde{Z}} \lim _{z \rightarrow 1} e^{\widetilde{\phi}} e^{2 \pi w_{\text {tot }} \widetilde{\xi}_{N}} \int \frac{d^{N} \sigma}{N !} \mathcal{Z}^{T[\mathrm{SU}(N)]}\left[\widetilde{\xi}_{1 \ldots N} ; \sigma_{j}-i w_{j} z\right] \\
& \times \frac{\prod_{i<j}^{N} \operatorname{sh}^{2}\left(\sigma_{i}-\sigma_{j}\right)}{\prod_{j}^{N} \operatorname{ch}\left(\sigma_{j}-i w_{j} z-\widetilde{m}_{2}\right) \operatorname{ch}\left(\sigma_{j}-\widetilde{m}_{1}\right)} \mathcal{Z}^{T[\operatorname{SU}(N)]}\left[-\widetilde{\xi}_{N+1 \ldots 2 N} ; \sigma_{j}\right],
\end{aligned}
$$

Plugging the explicit values (5.51) leads to

$$
\begin{aligned}
\widetilde{V}(w)= & \frac{1}{\widetilde{Z}} \lim _{z \rightarrow 1} \sum_{\tau, \tau^{\prime} \in S_{N}}(-1)^{\tau+\tau^{\prime}} \frac{e^{\widetilde{\phi}} e^{2 \pi w_{\text {tot }} \widetilde{\xi}_{N}}}{\prod\left[\widetilde{\xi}_{a}\right]} \int \frac{d^{N} \sigma}{N !} \frac{e^{2 \pi i \sum_{j}^{N} \sigma_{j}\left(\widetilde{\xi}_{\tau(j)}-\widetilde{\xi}_{\tau^{\prime}(j)+N}\right)}}{\prod_{j}^{N} \operatorname{ch}\left(\sigma_{j}-i w_{j} z-\widetilde{m}_{2}\right) \operatorname{ch}\left(\sigma_{j}-\widetilde{m}_{1}\right)} \\
& \times e^{2 \pi \sum_{j}^{N} \widetilde{\xi}_{\tau(j)} w_{j} z} \prod_{i<j}^{N} \frac{\operatorname{sh}\left(\sigma_{i}-\sigma_{j}\right)}{\operatorname{sh}\left(\sigma_{i}-\sigma_{j}-i\left(w_{i}-w_{j}\right) z\right)} .
\end{aligned}
$$


As we did already several times, we can plug directly $z=1$ in the factors on the second lign, since these factors do not have poles as $z$ goes from 0 to 1 . The ratio of sh factors

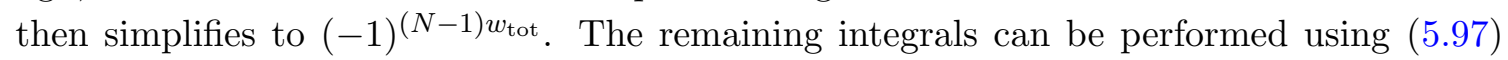
and the final result can be analytically continued to $z=1$. After simplification, we obtain

$$
\begin{aligned}
\widetilde{V}(w)= & \sum_{\tau, \tau^{\prime} \in S_{N}} \frac{(-1)^{\tau+\tau^{\prime}}}{\widetilde{Z}} \frac{(-i)^{N}(-1)^{N|\mathcal{R}|}}{N ! \prod\left[\widetilde{\xi}_{a}\right] \operatorname{sh}^{N}\left(\widetilde{m}_{1}-\widetilde{m}_{2}\right)} \\
& \times \prod_{j=1}^{N} \frac{e^{2 \pi i\left(\widetilde{m}_{1} \widetilde{\xi}_{\tau(j)}+\widetilde{m}_{2} \widetilde{\xi}_{\tau^{\prime}(j)+N}-i w_{j} \widetilde{\xi}_{\tau(j)}\right)}-e^{2 \pi i\left(\widetilde{m}_{2} \widetilde{\xi}_{\tau(j)}+\widetilde{m}_{1} \widetilde{\xi}_{\tau^{\prime}(j)+N}-i w_{j} \widetilde{\xi}_{\tau^{\prime}(j)+N}\right)}}{\operatorname{sh}\left(\widetilde{\xi}_{\tau(j)}-\widetilde{\xi}_{\tau^{\prime}(j)+N}\right)} .
\end{aligned}
$$

This matches precisely (5.99) upon identifying FI and mass parameters $\left(\xi_{1}, \xi_{2}, m_{a}\right)=$ $\left(\widetilde{m}_{1}, \widetilde{m}_{2}, \widetilde{\xi}_{a}\right)$, up to a sign, which was not carefully analyzed in the computations leading to the matrix models. This confirms the mirror symmetry prediction (4.15)

$$
\left\langle\widetilde{V}_{1, \mathcal{R}}^{(N)}\right\rangle \stackrel{\text { mirror }}{\longleftrightarrow}\left\langle W_{\mathcal{R}}\right\rangle \text {. }
$$

We notice that the partition functions $Z$ and $\widetilde{Z}$ can be found by setting to zero the weight $w$ and removing the normalization factors $Z^{-1}$ and $\widetilde{Z}^{-1}$ in the formula for $W(w)$ and $\widetilde{V}(w)$ respectively. We observe then that $Z$ and $\widetilde{Z}$ are exactly mapped under the identification $\left(\xi_{1}, \xi_{2}, m_{a}\right)=\left(\widetilde{m}_{1}, \widetilde{m}_{2}, \widetilde{\xi}_{a}\right)$. This would not have happened if we did not add the unphysical phases $e^{\phi}, e^{\widetilde{\phi}}$ to the matrix models.

\subsection{Hopping duality}

We have been claiming several times that each Vortex loop can be realized (at least) by two different $3 \mathrm{~d} / 1 \mathrm{~d}$ defect theories, which are read from the brane realization by moving the D1-branes to the closest NS5 on the left or on the right. The equivalence between the two defect theories is called hopping duality, in analogy with [21] (see also [8]). We can show that the $S^{3}$ partition function of the two defect theories indeed match.

Consider a Vortex loop $V_{M, \mathcal{R}}^{(N)}$ in a certain 3 d quiver theory, labeled by a representation $\mathcal{R}$ of a $\mathrm{U}(N)$ node and a splitting $K=M+(K-M)$ of the $K$ fundamental hypermultiplets of that node. It is realized by a brane configuration of figure 38-a, with $|\mathcal{R}|$ D1-branes ending on $N$ D3-branes with $K-M$ D5-branes on the left and $M$ D5-branes on the right. The associated left and right $3 \mathrm{~d} / 1 \mathrm{~d}$ theories are shown in 38 -b.

The matrix model associated to the right $3 \mathrm{~d} / 1 \mathrm{~d}$ theory and computing $\left\langle V_{M, \mathcal{R}}^{(N)}\right\rangle$ is given by (5.48) with the $1 \mathrm{~d}$ index (5.45):

$$
\begin{aligned}
\left\langle V_{M, \mathcal{R}}^{(N)}\right\rangle_{\text {right }}= & \lim _{z \rightarrow 1} \sum_{w \in \mathcal{R}} \frac{1}{Z} \int \frac{d^{N} \sigma}{N !} \frac{e^{2 \pi i\left(\xi_{l}-\xi_{r}\right) \sum_{j}^{N} \sigma_{j}} \prod_{i<j}^{N} \operatorname{sh}^{2}\left(\sigma_{i}-\sigma_{j}\right)}{\prod_{j}^{N}\left[\prod_{i}^{N_{l}} \operatorname{ch}\left(\sigma_{i}^{l}-\sigma_{j}\right) \prod_{a}^{K} \operatorname{ch}\left(\sigma_{j}-m_{a}\right) \prod_{k}^{N_{r}} \operatorname{ch}\left(\sigma_{k}-\sigma_{j}^{r}\right)\right]} \\
& \times e^{2 \pi \xi_{r}|\mathcal{R}|} \prod_{j}^{N}\left[\prod_{a=1}^{M} \frac{\operatorname{ch}\left(m_{a}-\sigma_{j}\right)}{\operatorname{ch}\left(m_{a}-\sigma_{j}-i w_{j} z\right)} \prod_{k=1}^{N_{r}} \frac{\operatorname{ch}\left(\sigma_{k}^{r}-\sigma_{j}\right)}{\operatorname{ch}\left(\sigma_{k}^{r}-\sigma_{j}-i w_{j} z\right)}\right][\cdots], \quad(5.104)
\end{aligned}
$$

where $[\cdots]$ indicates the matrix model associated to the other nodes of the $3 \mathrm{~d}$ quiver, which play no role in the check of the hopping duality, $\xi_{l}$ and $\xi_{r}$ are the "FI parameters" 
a)
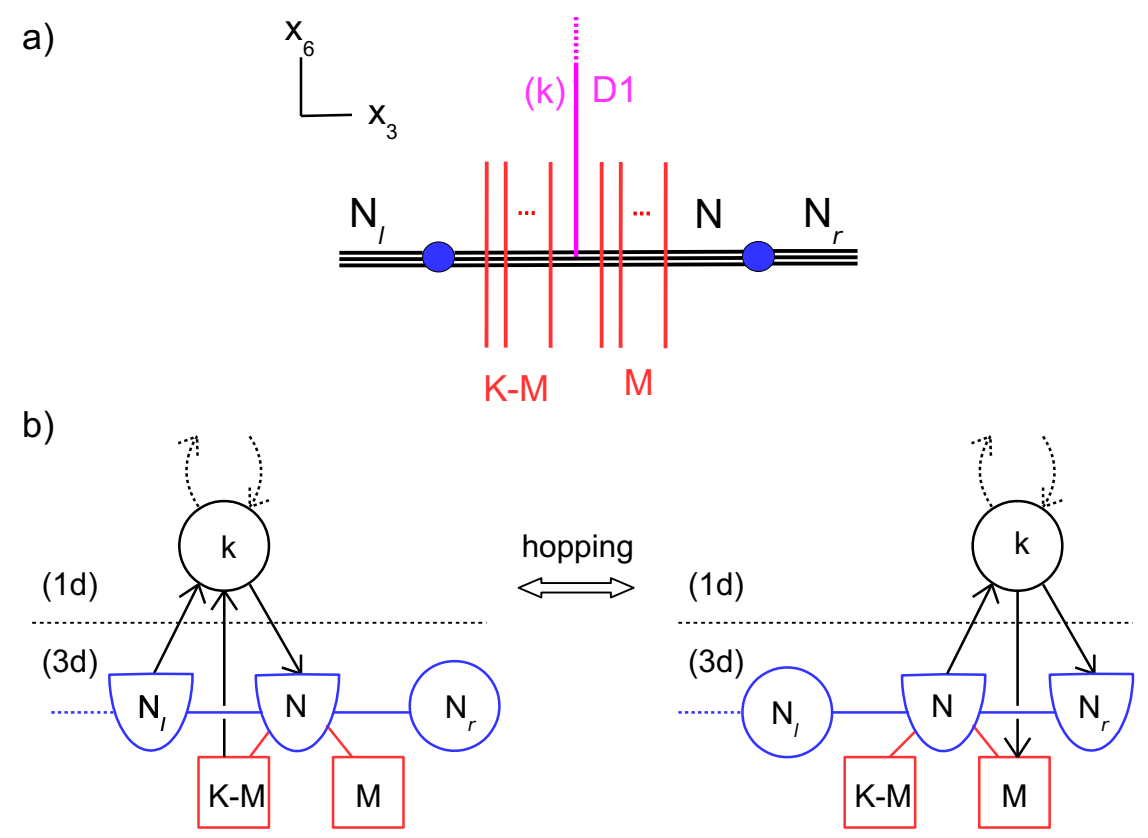

Figure 38. a) Brane realization of the Vortex loop $V_{M, \mathcal{R}}^{(N)}$. The $k=|\mathcal{R}|$ D1-branes end on NS5/NS5'branes which are not shown in the picture. b) Left and right 3d/1d quivers read from moving the D1-branes to the NS5 on the left or on the right, related by the hopping duality.

associated to the left and right NS5-branes $\left(\xi_{l}-\xi_{r}\right.$ is the FI parameter of the $\mathrm{U}(N)$ node), $m_{a}$ are the masses of the fundamental hypermultiplets and $\sigma_{j}^{l}$ and $\sigma_{k}^{r}$ are the eigenvalues of the $\mathrm{U}\left(N_{l}\right)$ and $\mathrm{U}\left(N_{r}\right)$ nodes standing respectively on the left and on the right of the $\mathrm{U}(N)$ node in the quiver diagram. This simplifies to

$$
\begin{aligned}
\left\langle V_{M, \mathcal{R}}^{(N)}\right\rangle_{\text {right }}= & \lim _{z \rightarrow 1} \sum_{w \in \mathcal{R}} \frac{1}{Z} \int \frac{d^{N} \sigma}{N !} \frac{e^{2 \pi i\left(\xi_{l}-\xi_{r}\right) \sum_{j}^{N} \sigma_{j}} \prod_{i<j}^{N} \operatorname{sh}^{2}\left(\sigma_{i}-\sigma_{j}\right)}{\prod_{j}^{N}\left[\prod_{i}^{N_{l}} \operatorname{ch}\left(\sigma_{i}^{l}-\sigma_{j}\right) \prod_{a=M+1}^{K} \operatorname{ch}\left(\sigma_{j}-m_{a}\right)\right]} \\
& \times \frac{e^{2 \pi \xi_{r}|\mathcal{R}|}}{\prod_{j}^{N}\left[\prod_{a=1}^{M} \operatorname{ch}\left(m_{a}-\sigma_{j}-i w_{j} z\right) \prod_{k=1}^{N_{r}} \operatorname{ch}\left(\sigma_{k}^{r}-\sigma_{j}-i w_{j} z\right)\right]}[\cdots] .
\end{aligned}
$$

The meaning of the $z \rightarrow 1$ limit, as explained after equation (5.48), is to take the analytical continuation of the matrix model computed with $i z \in \mathbb{R}$. We can thus perform the change of variable $\sigma_{j} \rightarrow \sigma_{j}-i w_{j} z$, leading to

$$
\begin{aligned}
\left\langle V_{M, \mathcal{R}}^{(N)}\right\rangle_{\text {right }}= & \lim _{z \rightarrow 1} \sum_{w \in \mathcal{R}} \frac{1}{Z} \int \frac{d^{N} \sigma}{N !} \frac{e^{2 \pi\left(\xi_{l}-\xi_{r}\right) z|\mathcal{R}|} e^{2 \pi i\left(\xi_{l}-\xi_{r}\right) \sum_{j}^{N} \sigma_{j}} \prod_{i<j}^{N} \operatorname{sh}^{2}\left(\sigma_{i}-\sigma_{j}-i\left(w_{i}-w_{j}\right) z\right)}{\prod_{j}^{N}\left[\prod_{i}^{N_{l}} \operatorname{ch}\left(\sigma_{i}^{l}-\sigma_{j}+i w_{j} z\right) \prod_{a=M+1}^{K} \operatorname{ch}\left(m_{a}-\sigma_{j}+i w_{j} z\right)\right]} \\
& \times \frac{e^{2 \pi \xi_{r}|\mathcal{R}|}}{\prod_{j}^{N}\left[\prod_{a=1}^{M} \operatorname{ch}\left(m_{a}-\sigma_{j}\right) \prod_{k=1}^{N_{r}} \operatorname{ch}\left(\sigma_{k}^{r}-\sigma_{j}\right)\right]}[\cdots]
\end{aligned}
$$

The analytical continuation $z \rightarrow 1$ can be taken directly in the integrand for the factors in the numerator, because they do not have poles for $z \in \mathbb{C}$. The expression then simplifies again and matches the matrix model computing $\left\langle V_{M, \mathcal{R}}^{(N)}\right\rangle$ from the left $3 \mathrm{~d} / 1 \mathrm{~d}$ theory 

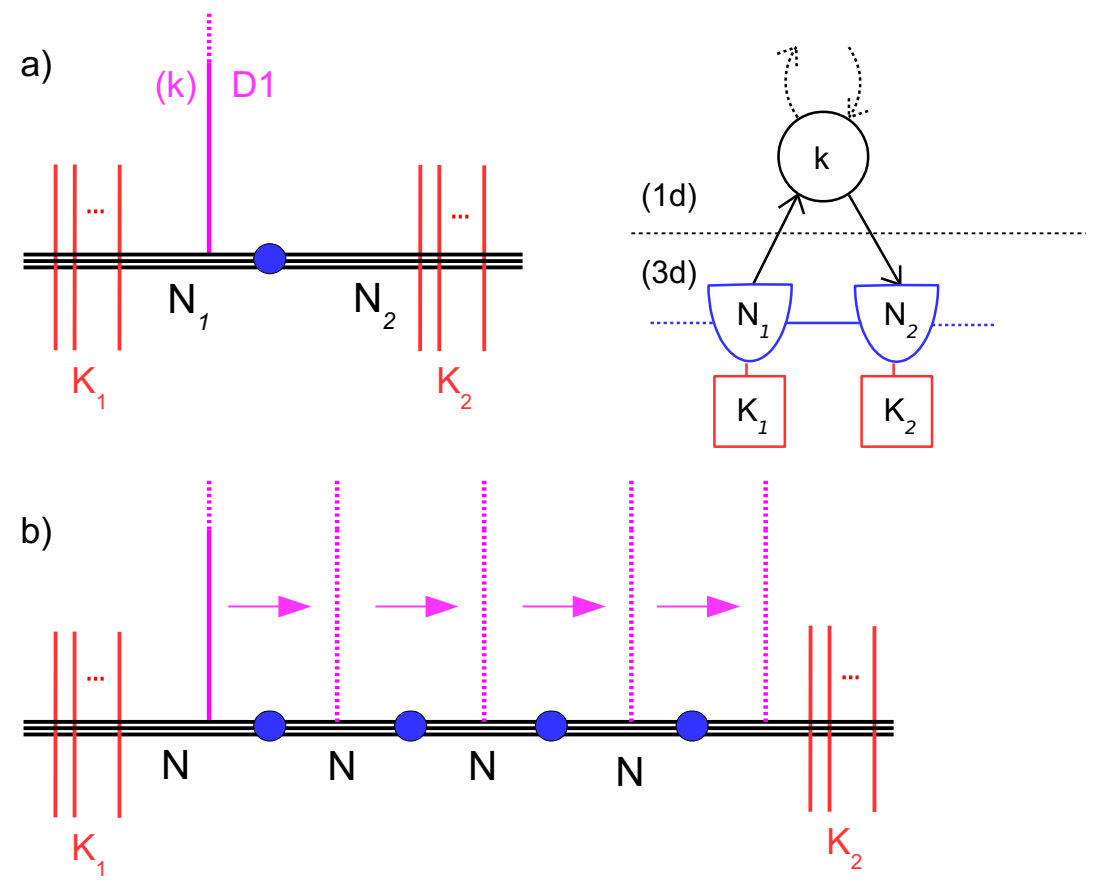

Figure 39. a) Brane realization of the Vortex loop $V_{0, \mathcal{R}_{1}}^{\left(N_{1}\right)}$ (with $k=\left|\mathcal{R}_{1}\right|$ ) and associated right 3d/1d theory. b) When the numbers of D3-branes on both sides of N5-branes are equal, namely the ranks of adjacent nodes are equal, the D1-branes can be moved across the NS5-branes. The Vortex loops they realize are all equivalent.

realization:

$$
\begin{aligned}
\left\langle V_{M, \mathcal{R}}^{(N)}\right\rangle_{\text {right }}= & \lim _{z \rightarrow 1} \sum_{w \in \mathcal{R}} \frac{1}{Z} \int \frac{d^{N} \sigma}{N !} \frac{e^{2 \pi \xi_{l}|\mathcal{R}|}}{\prod_{j}^{N}\left[\prod_{i}^{N_{l}} \operatorname{ch}\left(\sigma_{i}^{l}-\sigma_{j}+i w_{j} z\right) \prod_{a=M+1}^{K} \operatorname{ch}\left(m_{a}-\sigma_{j}+i w_{j} z\right)\right]} \\
& \times \frac{e^{2 \pi i\left(\xi_{l}-\xi_{r}\right) \sum_{j}^{N} \sigma_{j}} \prod_{i<j}^{N} \operatorname{sh}^{2}\left(\sigma_{i}-\sigma_{j}\right)}{\prod_{j}^{N}\left[\prod_{a=1}^{M} \operatorname{ch}\left(m_{a}-\sigma_{j}\right) \prod_{k=1}^{N_{r}} \operatorname{ch}\left(\sigma_{k}^{r}-\sigma_{j}\right)\right]}[\cdots] \\
= & \left\langle V_{M, \mathcal{R}}^{(N)}\right\rangle_{\text {left }},
\end{aligned}
$$

where we have used $\operatorname{sh}(x+i n)=(-1)^{n} \operatorname{sh}(x)$, for $n \in \mathbb{Z}$. This is indeed the matrix model associated to the left $3 \mathrm{~d} / 1 \mathrm{~d}$ theory given by (5.48) with the $1 \mathrm{~d}$ index (5.47), after simplification of some factors of ch. Note that the additional background Wilson loop is important to get a precise match.

The hopping duality also explains the equivalence of Vortex loops labeled by representations of different nodes. This occurs for instance for the Vortex loops of circular quivers with nodes of equal ranks described in section 4.4 (see (4.13), (4.14)).

Consider the quiver in figure 39-a with two adjacent nodes $\mathrm{U}\left(N_{1}\right)$ and $\mathrm{U}\left(N_{2}\right)$, with $K_{1}$ and $K_{2}$ fundamental hypermultiplets respectively and assume $N_{1} \geq N_{2}$. The vortex loop $V_{0, \mathcal{R}_{1}}^{\left(N_{1}\right)}$, with $\mathcal{R}_{1}$ a representation of $\mathrm{U}\left(N_{1}\right)$ and the subscript 0 indicating the splitting $K_{1}=0+\left(K_{1}-0\right)$, can be computed from the matrix model associated to the right $3 \mathrm{~d} / 1 \mathrm{~d}$ 
theory:

$$
\begin{aligned}
\left\langle V_{0, \mathcal{R}_{1}}^{\left(N_{1}\right)}\right\rangle_{\text {right }}= & \lim _{z \rightarrow 1} \sum_{w \in \mathcal{R}_{1}} \frac{1}{Z} \int \frac{d^{N_{1}} \sigma d^{N_{2}} \widetilde{\sigma}}{N_{1} ! N_{2} !} \prod_{i<j}^{N_{1}} \operatorname{sh}^{2}\left(\sigma_{i}-\sigma_{j}\right) \prod_{i<j}^{N_{2}} \operatorname{sh}^{2}\left(\widetilde{\sigma}_{i}-\widetilde{\sigma}_{j}\right) \\
& \times e^{2 \pi \xi_{r}\left|\mathcal{R}_{1}\right|} \prod_{j=1}^{N_{1}} \prod_{k=1}^{N_{2}} \frac{1}{\operatorname{ch}\left(\widetilde{\sigma}_{k}-\sigma_{j}-i w_{j} z\right)}[\cdots]
\end{aligned}
$$

where we indicated only the matrix factors inserted by the $1 \mathrm{~d}$ defect and part of sh factors of the two nodes, which will play a role in the check of hopping duality. Moreover the ch factors in the numerator of (5.45) have been canceled by the matrix factor of the $\mathrm{U}\left(N_{1}\right) \times \mathrm{U}\left(N_{2}\right)$ bifundamental hypermultiplet. This Vortex loop is realized by the brane configuration of figure 39-a with $\left|\mathcal{R}_{1}\right|$ D1-branes ending on the $N_{1}$ D3-branes supporting the $\mathrm{U}(N-1)$ node and standing to the right of the $K_{1}$ D5-branes.

In the simplest case when $N_{1}=N_{2}$, we have argued that the D1-branes can be moved to the right of the NS5-brane without changing the loop operator inserted. When D1branes stand on the right of the NS5-branes, they realize a Vortex loop $V_{K_{2}, \mathcal{R}_{2}}^{\left(N_{2}\right)}$ with $\mathcal{R}_{2}$ a representation of $\mathrm{U}\left(N_{2}\right)$ and the subscript $K_{2}$ indicating the splitting of $\mathrm{U}\left(N_{2}\right)$ fundamental hypermultiplets $K_{2}=K_{2}+\left(K_{2}-K_{2}\right)$. The left $3 \mathrm{~d} / 1 \mathrm{~d}$ theory associated to $V_{K_{2}, \mathcal{R}_{2}}^{\left(N_{2}\right)}$ is actually the same as the right $3 \mathrm{~d} / 1 \mathrm{~d}$ theory associated to $V_{0, \mathcal{R}_{1}}^{\left(N_{1}\right)}$, except for one important difference, which is that the FI parameter of the terminating SQM node (the node with $N_{1}$ fundamental and $N_{2}$ anti-fundamental chiral multiplets) is positive for $V_{K_{2}, \mathcal{R}_{2}}^{\left(N_{2}\right)}$ and negative for $V_{0, \mathcal{R}_{1}}^{\left(N_{1}\right)}$. The matrix model computing $\left\langle V_{K_{2}, \mathcal{R}_{2}}^{\left(N_{2}\right)}\right\rangle$ from the left $3 \mathrm{~d} / 1 \mathrm{~d}$ theory is given by

$$
\begin{aligned}
\left\langle V_{K_{2}, \mathcal{R}_{2}}^{\left(N_{2}\right)}\right\rangle_{\text {left }}= & \lim _{z \rightarrow 1} \sum_{w \in \mathcal{R}_{2}} \frac{1}{Z} \int \frac{d^{N_{1}} \sigma d^{N_{2}} \widetilde{\sigma}}{N_{1} ! N_{2} !} \prod_{i<j}^{N_{1}} \operatorname{sh}^{2}\left(\sigma_{i}-\sigma_{j}\right) \prod_{i<j}^{N_{2}} \operatorname{sh}^{2}\left(\widetilde{\sigma}_{i}-\widetilde{\sigma}_{j}\right) \\
& \times e^{2 \pi \xi_{r}\left|\mathcal{R}_{2}\right|} \prod_{j=1}^{N_{1}} \prod_{k=1}^{N_{2}} \frac{1}{\operatorname{ch}\left(\widetilde{\sigma}_{k}-i w_{k} z-\sigma_{j}\right)}[\cdots] .
\end{aligned}
$$

When $N_{1}=N_{2} \equiv N$ we must find $\left\langle V_{K_{2}, \mathcal{R}}^{\left(N_{2}\right)}\right\rangle=\left\langle V_{0, \mathcal{R}}^{\left(N_{1}\right)}\right\rangle$, as the D1-branes realizing the loops can be moved freely across the NS5. Let us consider this case first. Starting from the matrix model (5.108) computing $\left\langle V_{0, \mathcal{R}}^{\left(N_{1}\right)}\right\rangle$, with $N_{1}=N$, we can use the same trick as above and make the replacement $\prod_{i<j}^{N} \operatorname{sh}\left(\sigma_{i}-\sigma_{j}\right) \rightarrow(-1)^{(N-1)|\mathcal{R}|} \prod_{i<j}^{N} \operatorname{sh}\left(\sigma_{i}-\sigma_{j}+i\left(w_{i}-w_{j}\right) z\right)$ in the integrand. This allows us to use the Cauchy determinant formula (5.77) with $\sigma_{j} \rightarrow \sigma_{j}+i w_{j}$, leading to

$$
\begin{aligned}
\left\langle V_{0, \mathcal{R}}^{\left(N_{1}\right)}\right\rangle_{\text {right }}= & \lim _{z \rightarrow 1} \sum_{w \in \mathcal{R}} \sum_{\tau \in S_{N}}(-1)^{\tau} \frac{1}{Z} \int \frac{d^{N} \sigma d^{N} \widetilde{\sigma}}{N !^{2}} \prod_{i<j}^{N} \operatorname{sh}\left(\sigma_{i}-\sigma_{j}\right) \prod_{i<j}^{N} \operatorname{sh}\left(\widetilde{\sigma}_{i}-\widetilde{\sigma}_{j}\right) \\
& \times(-1)^{(N-1)|\mathcal{R}|} e^{2 \pi \xi_{r}|\mathcal{R}|} \frac{1}{\prod_{j}^{N} \operatorname{ch}\left(\sigma_{\tau(j)}+i w_{\tau(j)}-\widetilde{\sigma}_{j}\right)}[\cdots] .
\end{aligned}
$$


Relabeling $\sigma_{j} \rightarrow \sigma_{\tau^{-1}(j)}$ and $\widetilde{\sigma}_{j} \rightarrow \widetilde{\sigma}_{\tau(j)}$, we obtain

$$
\begin{aligned}
\left\langle V_{0, \mathcal{R}}^{\left(N_{1}\right)}\right\rangle_{\text {right }}= & \lim _{z \rightarrow 1} \sum_{w \in \mathcal{R}} \sum_{\tau \in S_{N}}(-1)^{\tau} \frac{1}{Z} \int \frac{d^{N} \sigma d^{N} \widetilde{\sigma}}{N !^{2}} \prod_{i<j}^{N} \operatorname{sh}\left(\sigma_{i}-\sigma_{j}\right) \prod_{i<j}^{N} \operatorname{sh}\left(\widetilde{\sigma}_{i}-\widetilde{\sigma}_{j}\right) \\
& \times(-1)^{(N-1)|\mathcal{R}|} e^{2 \pi \xi_{r}|\mathcal{R}|} \frac{1}{\prod_{j}^{N} \operatorname{ch}\left(\sigma_{j}+i w_{\tau(j)}-\widetilde{\sigma}_{\tau(j)}\right)}[\cdots] \\
= & \lim _{z \rightarrow 1} \sum_{w \in \mathcal{R}} \frac{1}{Z} \int \frac{d^{N} \sigma d^{N} \widetilde{\sigma}}{N !^{2}} \prod_{i<j}^{N} \operatorname{sh}^{2}\left(\sigma_{i}-\sigma_{j}\right) \prod_{i<j}^{N} \operatorname{sh}\left(\widetilde{\sigma}_{i}-\widetilde{\sigma}_{j}\right) \operatorname{sh}\left(\widetilde{\sigma}_{i}-\widetilde{\sigma}_{j}-i\left(w_{i}-w_{j}\right) z\right) \\
& \times(-1)^{(N-1)|\mathcal{R}|} e^{2 \pi \xi_{r}|\mathcal{R}|} \prod_{j=1}^{N_{1}} \prod_{k=1}^{N_{2}} \frac{1}{\operatorname{ch}\left(\widetilde{\sigma}_{k}-i w_{k} z-\sigma_{j}\right)}[\cdots] \\
= & \left\langle V_{K_{2}, \mathcal{R}}^{\left(N_{2}\right)}\right\rangle_{\text {left }},
\end{aligned}
$$

where we have used the Cauchy identity to obtain the second equality and we have again enforced $z=1$ in the sh factors of the integrand to reach the matrix model (5.109) computing $\left\langle V_{K_{2}, \mathcal{R}}^{\left(N_{2}\right)}\right\rangle$, with $N_{2}=N$.

This shows that the Vortex loops realized by D1-branes ending on the left or on the right of an NS5 with equal numbers of D3s on both sides are equivalent. Combining this property with the hopping duality between the left and right $3 \mathrm{~d} / 1 \mathrm{~d}$ quiver realization of a Vortex loop, we prove the equivalence of Vortex loops in circular quivers with nodes of equal rank (4.13), (4.14). This is illustrated in figure 39-b.

When $N_{1}>N_{2}$ the map of Vortex loops is more complicated. It can be found from the brane picture in the same way as we found mirror maps between loop operators. We consider the brane realization of $V_{0, \mathcal{R}_{1}}^{\left(N_{1}\right)}$ with $\left|\mathcal{R}_{1}\right|$ D1-strings ending on the $N_{1}$ D3-branes as in figure 39-a. As $N_{1}>N_{2}$, the NS5 has a D3-spike on its left side. We then move the D1-branes to the left, across the NS5-brane. Some D1-branes can be moved along the D3spike and get attached to the NS5-brane, realizing flavor Wilson loops for the U(1) global symmetry associated to the NS5-brane. The other D1-branes end on the $N_{2}$ D3-branes on the right of the NS5 and realize a Vortex loop $V_{K_{2}, \mathcal{R}_{2}}^{\left(N_{2}\right)}$. Recycling the ideas of section 3.2, the precise prediction from the brane picture is found to be

$$
\left\langle V_{0, \mathcal{R}_{1}}^{\left(N_{1}\right)}\right\rangle=\left\langle\sum_{s \in \Delta} W_{N S 5, \mathbf{q}_{s}}^{\mathrm{f}} V_{K_{2}, \widehat{\mathcal{R}}_{s}}^{\left(N_{2}\right)}\right\rangle,
$$

where $\Delta$ is the set of representations $\left(\mathbf{q}_{s}, \widehat{\mathcal{R}}_{s}\right)$ appearing in the decomposition of $\mathcal{R}_{1}$ under the subgroup $\mathrm{U}(1) \times \mathrm{U}\left(N_{2}\right) \subset \mathrm{U}\left(N_{1}-N_{2}\right) \times \mathrm{U}\left(N_{2}\right) \subset \mathrm{U}\left(N_{1}\right)$, with $\mathrm{U}(1)$ embedded diagonally into $\mathrm{U}\left(N_{1}-N_{2}\right)$. $W_{N S 5, \mathbf{q}}^{\mathrm{fl}}$ denotes the Wilson loop of charge $\mathbf{q}$ under the $\mathrm{U}(1)$ topological symmetry associated to the NS5.

This map can be checked by explicit computations, using the generalized Cauchy formula (5.78). It implies that the Vortex loops $V_{0, \mathcal{R}_{1}}^{\left(N_{1}\right)}$ and $V_{K_{2}, \mathcal{R}_{2}}^{\left(N_{2}\right)}$ are redundant and that in order to describe the mirror map with Wilson loops of the mirror theory, it is sufficient to consider only the loops $V_{0, \mathcal{R}_{1}}^{\left(N_{1}\right)}$ or the loops $V_{K_{2}, \mathcal{R}_{2}}^{\left(N_{2}\right)}$. In general for each pair of consecutive 
D5-branes in the brane picture, we need only to consider the Vortex loops realized with D1branes placed between the two D5-branes, with a fixed number of NS5-branes on their left and on their right. This is the mirror statement to the fact that between two consecutive NS5-branes, we need only consider Wilson loops realized with F1-strings placed between the two NS5s, with a fixed number of D5-branes on their left and on their right.

\section{Acknowledgments}

We would like to thank Kevin Costello, Nadav Drukker, Davide Gaiotto, Heeyeon Kim, Hee-Cheol Kim, Bruno Le Floch, Stefano Cremonesi, R. Santamaria and E. Witten for discussions. B.A. thanks Perimeter Institute for its generous hospitality during several visits between 2013 and 2015. JG is grateful to the KITP for its warm hospitality during the "New Methods in Nonperturbative Quantum Field Theory" Program in early 2014, which was supported in part by the National Science Foundation under Grant No. NSF PHY11-25915. This research was supported in part by Perimeter Institute for Theoretical Physics. Research at Perimeter Institute is supported by the Government of Canada through Industry Canada and by the Province of Ontario through the Ministry of Research and Innovation. J.G. also acknowledges further support from an NSERC Discovery Grant and from an ERA grant by the Province of Ontario. B.A. acknowledges support by the ERC Starting Grant N. 304806, "The Gauge/Gravity Duality and Geometry in String Theory".

\section{A Supersymmetry transformations on $S^{3}$ and SQM embedding}

We provide here the $3 \mathrm{~d} \mathcal{N}=4$ supersymmetry transformations of the vector multiplet and hypermultiplet on $S^{3}$. By restricting to the four supercharges preserved by the $\mathrm{SQM}_{V}$ deformed algebra $\mathrm{SU}(1 \mid 1)_{l} \times \mathrm{SU}(1 \mid 1)_{R}$ in (5.19)(5.20), we work out the embedding of the $1 \mathrm{~d} \mathcal{N}=4$ vector and chiral multiplets inside the $3 \mathrm{~d} \mathcal{N}=4$ vector and hypermultiplets.

The $3 \mathrm{~d} \mathcal{N}=4$ supersymmetry transformations are generated by the four two-component Killing spinors $\epsilon^{A A^{\prime}}$, with $A=1,2$ the index of the $\mathbf{2}$ of $\mathrm{SU}(2)_{C}$ and $A^{\prime}=1,2$ the index of the $\mathbf{2}$ of $\mathrm{SU}(2)_{H}$. The transformations of the $3 \mathrm{~d} \mathcal{N}=4$ abelian vector multiplet $\left(A_{\mu}, \Phi_{I}, \lambda^{A A^{\prime}}, \mathrm{D}_{I^{\prime}}\right)$ are $^{72}$

$$
\begin{aligned}
\delta A_{\mu} & =-\frac{i}{2} \lambda_{A A^{\prime}} \gamma_{\mu} \epsilon^{A A^{\prime}} \quad \delta \Phi_{I}=-\frac{1}{2} \lambda_{A A^{\prime}}\left(\tau_{I}\right)^{A}{ }_{B} \epsilon^{B A^{\prime}} \\
\delta \lambda^{A A^{\prime}} & =-\frac{1}{2} F_{\mu \nu} \gamma^{\mu \nu} \epsilon^{A A^{\prime}}-\mathrm{D}_{I^{\prime}}\left(\tau_{I^{\prime}}\right)^{A^{\prime}}{ }_{B^{\prime}} \epsilon^{A B^{\prime}}+i D_{\mu} \Phi_{I} \gamma^{\mu}\left(\tau_{I}\right)^{A}{ }_{B} \epsilon^{B A^{\prime}}+\frac{2 i}{3} \Phi_{I} \gamma^{\mu}\left(\tau_{I}\right)^{A}{ }_{B} D_{\mu} \epsilon^{B A^{\prime}} \\
\delta \mathrm{D}_{I^{\prime}} & =-\frac{i}{2} D_{\mu} \lambda_{A A^{\prime}} \gamma_{\mu}\left(\tau_{I^{\prime}}\right)^{A^{\prime}}{ }_{B^{\prime}} \epsilon^{A B^{\prime}}-\frac{i}{6} \lambda_{A A^{\prime}} \gamma_{\mu}\left(\tau_{I^{\prime}}\right)^{A^{\prime}}{ }_{B^{\prime}} D_{\mu} \epsilon^{A B^{\prime}},
\end{aligned}
$$

where $\tau_{I}=\tau_{I^{\prime}}$ are the Pauli matrices. $I=1,2,3$ is the index of the $\mathbf{3}$ of $\mathrm{SU}(2)_{C}$ while $I^{\prime}=1,2,3$ is the index of the 3 of $\mathrm{SU}(2)_{H}$. The Killing spinors $\epsilon^{11}=\bar{\epsilon}_{L}$ and $\epsilon^{22}=\epsilon_{L}$ generate the $3 \mathrm{~d} \mathcal{N}=2$ supersymmetry transformations in $\mathrm{SU}(2 \mid 1)_{l}$, while $\epsilon^{12}=\bar{\epsilon}_{R}$ and $\epsilon^{21}=\epsilon_{R}$ generate supersymmetry transformations in $\mathrm{SU}(2 \mid 1)_{r}$.

\footnotetext{
${ }^{72}$ We derived these transformations by "covariantizing" the $3 \mathrm{~d} \mathcal{N}=2$ transformations of [4].
} 
The $3 \mathrm{~d} \mathcal{N}=4$ vector multiplet decomposes into a $3 \mathrm{~d} \mathcal{N}=2$ vector multiplet $\left(A_{\mu}, \Phi_{3}, \lambda^{11}, \lambda^{22}, \mathrm{D}_{3}\right)=\left(A_{\mu}, \sigma, \lambda, \bar{\lambda}, D\right)$ and an adjoint chiral multiplet $\left(\Phi_{1}+\right.$ $\left.i \Phi_{2}, \lambda^{12}, \lambda^{21}, \mathrm{D}_{1}+i \mathrm{D}_{2}\right)=(\phi, \psi, \bar{\psi}, F)$.

$\mathrm{SQM}_{V}$ embedding. The four Killing spinors generating the deformed $\mathrm{SQM}_{V}$ algebra are (see section 5.2)

$$
\epsilon_{l}=\left(\begin{array}{c}
\epsilon_{1} \\
0
\end{array}\right), \quad \bar{\epsilon}_{l}=\left(\begin{array}{c}
0 \\
\bar{\epsilon}_{2}
\end{array}\right), \quad \epsilon_{r}=\left(\begin{array}{c}
0 \\
\tilde{\epsilon}_{2}
\end{array}\right), \quad \bar{\epsilon}_{r}=\left(\begin{array}{c}
\overline{\tilde{\epsilon}}_{1} \\
0
\end{array}\right) .
$$

In the left-invariant frame, $\epsilon_{l}$ and $\bar{\epsilon}_{l}$ are constant, while $\epsilon_{r}$ and $\bar{\epsilon}_{r}$ have a spatial dependent phase $e^{i \tau / L}$ and $e^{-i \tau / L}$ respectively, where $\tau \in[0,2 \pi L]$ is the coordinate along the loop. We recall that the equations solved by the Killing spinors are

$$
\begin{aligned}
\nabla_{\mu} \epsilon_{l} & =\frac{i}{2 L} \gamma_{\mu} \epsilon_{l}, & \nabla_{\mu} \bar{\epsilon}_{l} & =\frac{i}{2 L} \gamma_{\mu} \bar{\epsilon}_{l}, \\
\nabla_{\mu} \epsilon_{r} & =-\frac{i}{2 L} \gamma_{\mu} \epsilon_{r}, & \nabla_{\mu} \bar{\epsilon}_{r} & =-\frac{i}{2 L} \gamma_{\mu} \bar{\epsilon}_{r},
\end{aligned}
$$

where $L$ is the radius of $S^{3}$. The supersymmetry transformations under the supercharges in deformed superalgebra $\mathrm{SQM}_{V}$ reduce to ${ }^{73}$

$$
\begin{aligned}
\delta A_{\tau} & =-\frac{i}{2}\left(\bar{\lambda}_{1} \bar{\epsilon}_{2}+\lambda_{2} \epsilon_{1}-\bar{\psi}_{2} \overline{\tilde{\epsilon}}_{1}-\psi_{1} \tilde{\epsilon}_{2}\right) \\
\delta \Phi_{3} & =-\frac{1}{2}\left(-\bar{\lambda}_{1} \bar{\epsilon}_{2}-\lambda_{2} \epsilon_{1}-\bar{\psi}_{2} \overline{\tilde{\epsilon}}_{1}-\psi_{1} \tilde{\epsilon}_{2}\right) \\
\delta \Phi & =\bar{\lambda}_{1} \tilde{\epsilon}_{2}+\bar{\psi}_{2} \epsilon_{1} \quad \delta \bar{\Phi}=-\lambda_{2} \overline{\tilde{\epsilon}}_{1}-\psi_{1} \bar{\epsilon}_{2} \\
\delta \bar{\lambda}_{1} & =\left(\mathrm{D}_{3}-\frac{i}{2} F_{12}+\frac{\Phi_{3}}{L}\right) \epsilon_{1}-i D_{\tau} \Phi_{3} \epsilon_{1}+i\left(D_{\tau} \Phi-i \frac{\Phi}{L}\right) \overline{\tilde{\epsilon}}_{1} \\
\delta \lambda_{2} & =-\left(\mathrm{D}_{3}-\frac{i}{2} F_{12}+\frac{\Phi_{3}}{L}\right) \bar{\epsilon}_{2}-i D_{\tau} \Phi_{3} \bar{\epsilon}_{2}-i\left(D_{\tau} \bar{\Phi}+i \frac{\bar{\Phi}}{L}\right) \tilde{\epsilon}_{2} \\
\delta \psi_{1} & =\left(\mathrm{D}_{3}-\frac{i}{2} F_{12}+\frac{\Phi_{3}}{L}\right) \overline{\tilde{\epsilon}}_{1}+i D_{\tau} \Phi_{3} \overline{\tilde{\epsilon}}_{1}+i\left(D_{\tau} \bar{\Phi}+i \frac{\bar{\Phi}}{L}\right) \epsilon_{1} \\
\delta \bar{\psi}_{2} & =-\left(\mathrm{D}_{3}-\frac{i}{2} F_{12}+\frac{\Phi_{3}}{L}\right) \tilde{\epsilon}_{2}+i D_{\tau} \Phi_{3} \tilde{\epsilon}_{2}-i\left(D_{\tau} \Phi-i \frac{\Phi}{L}\right) \bar{\epsilon}_{2},
\end{aligned}
$$

where $\Phi=\Phi_{1}+i \Phi_{2}$ and we have adopted $3 \mathrm{~d} \mathcal{N}=2$ notations $\lambda_{\alpha}, \bar{\lambda}_{\alpha}, \psi_{\alpha}, \bar{\psi}_{\alpha}$ for the fermions, with $\alpha=1,2$.

These correspond to the supersymmetry transformations of a $1 \mathrm{~d} \mathcal{N}=4 \mathrm{SQM}_{V}$ abelian vector multiplet (see for instance [18]) but in the presence of a background gauge field $a_{-}=-\frac{1}{L}$ for the $\mathrm{U}(1)_{-}$R-symmetry, generated by $J_{-}$. The presence of the background gauge field $a_{-}=-\frac{1}{L}$ affects the covariant derivative of $\Phi, \bar{\Phi}$ in the transformations above. The identification of the fields in the $3 \mathrm{~d} \mathcal{N}=4$ vector multiplet with the fields of the $1 \mathrm{~d} \mathcal{N}=2$ vector and adjoint chiral multiplet that furnish a $1 \mathrm{~d} \mathcal{N}=4 \mathrm{SQM}_{V}$ vector

\footnotetext{
${ }^{73}$ We do not give the supersymmetry transformations of the auxiliary fields and transverse gauge fields, which combine into another $1 \mathrm{~d} \mathcal{N}=4$ chiral multiplet.
} 
multiplet is

1d vector multiplet : $\left(v_{\tau}, x_{3}, \lambda_{-}, \bar{\lambda}_{-}, D\right)_{1 d} \sim\left(A_{\tau}, \Phi_{3}, \lambda_{2}, \bar{\lambda}_{1}, \mathrm{D}_{3}-\frac{i}{2} F_{12}+\frac{\Phi_{3}}{L}\right), \quad r_{-}=0$

1d chiral multiplet : $\quad\left(x_{1}+i x_{2}, \psi, \bar{\psi}\right)_{1 d} \sim\left(\Phi, \bar{\psi}_{2}, \psi_{1}\right), \quad r_{-}=-1$,

where we have indicated the $\mathrm{U}(1)$ - charges of the $1 \mathrm{~d} \mathcal{N}=2$ multiplets: ${ }^{74}$ the $1 \mathrm{~d} \mathcal{N}=2$ vector multiplet fields have vanishing $\mathrm{U}(1)_{-}$charge, while the fields $x_{1}+i x_{2}$ and $\psi$ in the $1 \mathrm{~d} \mathcal{N}=2$ SQM adjoint chiral multiplet have $\mathrm{U}(1)_{\text {- charge }}-1$ and $\bar{\psi}$ charge 1 .

We now turn to the $3 \mathrm{~d} \mathcal{N}=4$ hypermultiplet supersymmetry transformations. These do not admit an off-shell formulation, so we provide the on-shell supersymmetry transformations:

$$
\begin{aligned}
\delta \phi^{A^{\prime}} & =-\psi_{A} \epsilon^{A A^{\prime}} \\
\delta \psi^{A} & =i \gamma^{\mu} \epsilon^{A A^{\prime}} D_{\mu} \phi_{A^{\prime}}+i \Phi_{I}\left(\tau_{I}\right)^{A}{ }_{B} \phi_{A^{\prime}} \epsilon^{B A^{\prime}}+\frac{i}{3} \gamma^{\mu} D_{\mu} \epsilon^{A A^{\prime}} \phi_{A^{\prime}} .
\end{aligned}
$$

$\left(\phi^{1},-\psi^{2}\right)$ and $\left(\phi^{2}, \psi^{1}\right)$ are two on-shell chiral multiplets of $3 \mathrm{~d} \mathcal{N}=2$ supersymmetry of R-charge $1 / 2$ transforming under complex conjugate representations of the gauge group.

Restricting to the same four supercharges generating deformed $\mathrm{SQM}_{V}$ as above, we obtain

$$
\begin{aligned}
\delta \phi^{1} & =\left(\psi^{1}\right)_{1} \tilde{\epsilon}_{2}-\left(\psi^{2}\right)_{1} \bar{\epsilon}_{2} \\
\delta\left(\psi^{1}\right)_{1} & =i \overline{\tilde{\epsilon}}_{1} D_{\tau} \phi^{1}+i\left(\Phi_{3}-\frac{i}{2 L}\right) \phi^{1} \overline{\tilde{\epsilon}}_{1}+i \bar{\Phi} \phi^{1} \epsilon_{1}-i\left(D_{1}-i D_{2}\right) \phi^{2} \bar{\epsilon}_{2} \\
\delta\left(\psi^{2}\right)_{1} & =i \epsilon_{1} D_{\tau} \phi^{1}-i\left(\Phi_{3}-\frac{i}{2 L}\right) \phi^{1} \epsilon_{1}+i \Phi \phi^{1} \overline{\tilde{\epsilon}}_{1}-i\left(D_{1}-i D_{2}\right) \phi^{2} \tilde{\epsilon}_{2} \\
\delta \phi^{2} & =\left(\psi^{1}\right)_{2} \epsilon_{1}-\left(\psi^{2}\right)_{2} \overline{\tilde{\epsilon}}_{1} \\
\delta\left(\psi^{1}\right)_{2} & =-i \bar{\epsilon}_{2} D_{\tau} \phi^{2}+i\left(\Phi_{3}+\frac{i}{2 L}\right) \phi^{2} \bar{\epsilon}_{2}+i \bar{\Phi} \phi^{2} \tilde{\epsilon}_{2}-i\left(D_{1}+i D_{2}\right) \phi^{1} \overline{\tilde{\epsilon}}_{1} \\
\delta\left(\psi^{2}\right)_{2} & =-i \tilde{\epsilon}_{2} D_{\tau} \phi^{2}-i\left(\Phi_{3}+\frac{i}{2 L}\right) \phi^{2} \tilde{\epsilon}_{2}+i \Phi \phi^{2} \bar{\epsilon}_{2}-i\left(D_{1}+i D_{2}\right) \phi^{1} \epsilon_{1} .
\end{aligned}
$$

This matches the on-shell deformed supersymmetry transformations of two $1 \mathrm{~d} \mathcal{N}=4$ chiral multiplets $\left(\phi, \psi_{+}, \psi_{-}\right)_{q_{F}} \sim\left(\phi^{1},\left(\psi^{1}\right)_{1},\left(\psi^{2}\right)_{1}\right)_{-\frac{1}{2}}$ and $\left(\phi^{2},\left(\psi^{1}\right)_{2},\left(\psi^{2}\right)_{2}\right)_{\frac{1}{2}}$. The shift of $\Phi_{3}$ in the supersymmetry transformations is identified with a real mass deformation with complex parameter $m_{F}=\frac{i}{L}$ for a flavor symmetry $G_{F}$, such that the $1 \mathrm{~d} \mathcal{N}=4$ chiral multiplets have $G_{F}$ charge $q_{F}=\mp \frac{1}{2}$. The term $\left(D_{1}+i D_{2}\right) \phi^{1}$ in the on-shell $1 \mathrm{~d} \mathcal{N}=4$ supersymmetry transformations arises from a superpotential coupling. In the off-shell transformations it gets replaced with a complex auxiliary field.

We note that the mass deformation obtained by giving a background to the $G_{F}$ flavour symmetry is not visible anywhere in the transformations (A.4), which means that the

\footnotetext{
${ }^{74} \mathrm{U}(1)_{-}$is a global symmetry from the point of view of the $1 \mathrm{~d} \mathcal{N}=2$ subalgebra, so that each $\mathcal{N}=2$ multiplet comes with a $\mathrm{U}(1)_{-}$charge.
} 
adjoint chiral multiplet with bottom component $\Phi=\Phi_{1}+i \Phi_{2}$ is not charged under $G_{F}$. This allows us to identify this flavor symmetry with

$$
G_{F}=J_{3}^{l}+J_{3}^{r}-R_{H} .
$$

Moreover the background gauge field $a_{-}=-\frac{1}{L}$ for $J_{-}$is not visible in the transformations (A.9) of the chiral multiplets with bottom components $\phi^{1}$ and $\phi^{2}$, which means that these multiplets are not charged under $J_{-}$. We then have the identification

$$
J_{-}=J_{3}^{l}+J_{3}^{r}-R_{V} \text {. }
$$

This identification of generators allows us to match the deformed $\mathrm{SQM}_{W}$ supersymmetry algebra with the $\mathrm{SU}(1 \mid 1)_{l} \times \mathrm{SU}(1 \mid 1)_{r}$ subalgebra preserved by the defect, as explained in section 5.2 .

\section{B Evaluations of the SQM index}

In this appendix we compute the $1 \mathrm{~d} \mathcal{N}=4 \mathrm{SQM}$ index, or partition function on $S^{1}$, for some quiver gauge theories with generic $\mathrm{U}(1)_{-}$R-symmetry background $z$ and $\mathrm{U}(1)_{F}$ flavor chemical potential $\mu=1$, as defined in section 5.3.2. The R-symmetry and flavor symmetry charges are summarized in table 6 and the specific adjoint R-charges are given in equation (5.43).

\section{B.1 $\mathrm{U}(k)$ theory with $N$ fundamental and $M$ anti-fundamental chirals}

We consider a $1 \mathrm{~d} \mathcal{N}=4 \mathrm{SQM}$ gauge theory with $\mathrm{U}(k)$ gauge group and $N$ fundamental chiral multiplets with real masses $-\sigma_{j}$, R-charges $r_{+}$and $G_{F}$ flavor charge $q_{+}$, and $M$ anti-fundamental chiral multiplets with masses $m_{a}$, R-charges $r_{-}$and $G_{F}$ flavor charge $q_{-}$. Mass parameters are in units of the inverse $S^{1}$ radius. As explained in the main text the charges obey the superpotential constraints $r_{-}+r_{+}=2$ and $q_{-}+q_{+}=1 / 2$. We introduce the complex parameters $\hat{\sigma}_{j}=\sigma_{j}+i q_{+}+i \frac{r_{+}}{2} z$ and $\hat{m}_{a}=m_{a}-i q_{-}-i \frac{r_{-}}{2} z$. Importantly we take a negative FI parameter $\zeta<0$. This corresponds to the choice of FI parameter when the $1 \mathrm{~d} \mathcal{N}=4$ theory is read by moving the D1-branes to the right NS5-brane.

The partition function is given by

$$
\begin{aligned}
\mathcal{I}= & J K-\operatorname{Res}_{\zeta} \frac{1}{k !}\left(\frac{\pi}{\sin (\pi z)}\right)^{k} \prod_{I \neq J}^{k} \frac{\sin \left[-\pi\left(u_{I}-u_{J}\right)\right]}{\sin \left[\pi\left(u_{I}-u_{J}-z\right)\right]} \\
& \times \prod_{I=1}^{k}\left(\prod_{j=1}^{N} \frac{\sin \left[-\pi\left(u_{I}-i \hat{\sigma}_{j}-z\right)\right]}{\sin \left[\pi\left(u_{I}-i \hat{\sigma}_{j}\right)\right]} \prod_{a=1}^{M} \frac{\sin \left[-\pi\left(-u_{I}+i \hat{m}_{a}-z\right)\right]}{\sin \left[\pi\left(-u_{I}+i \hat{m}_{a}\right)\right]} d u_{I}\right) \\
= & \frac{1}{k !}(2 i \sin (\pi z))^{-k} \oint \prod_{I=1}^{k} d u_{I} \prod_{I \neq J}^{k} \frac{\sin \left[-\pi\left(u_{I}-u_{J}\right)\right]}{\sin \left[\pi\left(u_{I}-u_{J}-z\right)\right]} \\
& \times \prod_{I=1}^{k}\left(\prod_{j=1}^{N} \frac{\sin \left[-\pi\left(u_{I}-i \hat{\sigma}_{j}-z\right)\right]}{\sin \left[\pi\left(u_{I}-i \hat{\sigma}_{j}\right)\right]} \prod_{a=1}^{M} \frac{\sin \left[-\pi\left(-u_{I}+i \hat{m}_{a}-z\right)\right]}{\sin \left[\pi\left(-u_{I}+i \hat{m}_{a}\right)\right]}\right),
\end{aligned}
$$


where the integration contour is defined so that it picks the residues at the poles from the fundamental chiral multiplet factors, namely factors in the product $\prod_{j=1}^{N}$. In particular it does not pick the poles from factors in the product $\prod_{I \neq J}^{k}$ and $\prod_{a=1}^{M}$. This follows from the definition of $\mathrm{JK}-\operatorname{Res}_{\zeta}$ for $\zeta<0$, reviewed in the main text. This integral has poles at $u_{I}^{*}=i \hat{\sigma}_{j}, j=1, \cdots, N$, however, due to the $\sin \left[-\pi\left(u_{I}-u_{J}\right)\right]$ factors, we get a non-zero residue only when each $u_{I}^{*}$ hits a different $i \hat{\sigma}_{j}$, in particular we have non-zero residues only when $k \leq N$. A non-vanishing residue at $u^{*}=\left\{u_{I}^{*}\right\}_{1 \leq I \leq k}$ is then associated to a decomposition $k=\sum_{k=1}^{N} k_{j}$, with $k_{1}, \cdots, k_{N} \in\{0,1\}$, where $k_{j}=1$ if $u_{I}^{*}=i \hat{\sigma}_{j}$ for a given $I$ and $k_{j}=0$ otherwise. This residue contribution appears with a multiplicity $k$ !, corresponding to permutations of the $u_{I}^{*}$. The partition function is then expressed as

$$
\mathcal{I}=\sum_{\substack{k_{1}, \cdots, k_{N} \in\{0,1\} \\ \sum_{k=1}^{N} k_{j}=k}} \mathcal{I}_{\left(k_{j}\right)},
$$

with

$$
\mathcal{I}_{\left(k_{j}\right)}=(-1)^{(N+M) k} \prod_{i<j}^{N} \frac{\operatorname{sh}\left[\hat{\sigma}_{i}-\hat{\sigma}_{j}+i\left(k_{i}-k_{j}\right) z\right]}{\operatorname{sh}\left[\hat{\sigma}_{i}-\hat{\sigma}_{j}\right]} \prod_{a=1}^{M} \prod_{j=1}^{N} \frac{\operatorname{sh}\left(\hat{\sigma}_{j}-\hat{m}_{a}-i z\right)}{\operatorname{sh}\left(\hat{\sigma}_{j}-\hat{m}_{a}+i\left(k_{j}-1\right) z\right)}
$$

The partition function vanishes when $k>N$, consistent with the fact that there are no supersymmetry vacua in that range.

Plugging the constraints $r_{+}+r_{-}=2$ and $q_{+}+q_{-}=1 / 2$ we obtain

$$
\mathcal{I}_{\left(k_{j}\right)}=(-1)^{(N+M) k} \prod_{i<j}^{N} \frac{\operatorname{sh}\left[\sigma_{i}-\sigma_{j}+i\left(k_{i}-k_{j}\right) z\right]}{\operatorname{sh}\left[\sigma_{i}-\sigma_{j}\right]} \prod_{j=1}^{N} \prod_{a=1}^{M} \frac{\operatorname{ch}\left(\sigma_{j}-m_{a}\right)}{\operatorname{ch}\left(\sigma_{j}-m_{a}+i k_{j} z\right)}
$$

This result matches the formula (5.45) giving the index as a sum over the weights of the representation $\mathcal{A}_{k}$ associated to the $1 \mathrm{~d} \mathcal{N}=4$ gauge theory.

\section{B.2 $\mathrm{U}(k)$ theory with $N$ fundamental, $M$ anti-fundamental chirals and one adjoint chiral}

We consider a $1 \mathrm{~d} \mathcal{N}=4 \mathrm{SQM}$ gauge theory with $\mathrm{U}(k)$ gauge group and $N$ fundamental chiral multiplets with real masses $-\sigma_{j}$, R-charges $r_{+}$and $G_{F}$ flavor charge $q_{+}, M$ antifundamental chiral multiplets with masses $m_{a}$, R-charges $r_{-}$and $G_{F}$ flavor charge $q_{-}$and an adjoint chiral multiplet with R-charge $R_{\text {adj }}=2$ and flavor charge $q_{\text {adj }}=1$. Mass parameters are in units of the inverse $S^{1}$ radius. The charges obey the superpotential constraints $r_{-}+r_{+}=2$ and $q_{-}+q_{+}=1 / 2$. We introduce the complex parameters $\hat{\sigma}_{j}=\sigma_{j}+i q_{+}+i \frac{r_{+}}{2} z$ and $\hat{m}_{a}=m_{a}-i q_{-}-i \frac{r_{-}}{2} z$. Importantly we have a negative FI parameter $\zeta<0$. 
In order to avoid higher order poles in the computation we keep $R_{\text {adj }}$ generic and only set it to 2 at the end of the computation. The partition function or index is given by

$$
\begin{aligned}
\mathcal{I}= & J K-\operatorname{Res}_{\zeta} \frac{1}{k !}\left(\frac{\pi}{\sin (\pi z)}\right)^{k} \prod_{I \neq J}^{k} \frac{\sin \left[-\pi\left(u_{I}-u_{J}\right)\right]}{\sin \left[\pi\left(u_{I}-u_{J}-z\right)\right]} \prod_{I, J}^{k} \frac{\sin \left[-\pi\left(u_{I}-u_{J}+\frac{R_{\mathrm{adj}}}{2} z-z\right)\right]}{\sin \left[\pi\left(u_{I}-u_{J}+\frac{R_{\mathrm{adj}}}{2} z\right)\right]} \\
& \times \prod_{I=1}^{k}\left(\prod_{j=1}^{N} \frac{\sin \left[-\pi\left(u_{I}-i \hat{\sigma}_{j}-z\right)\right]}{\sin \left[\pi\left(u_{I}-i \hat{\sigma}_{j}\right)\right]} \prod_{a=1}^{M} \frac{\sin \left[-\pi\left(-u_{I}+i \hat{m}_{a}-z\right)\right]}{\sin \left[\pi\left(-u_{I}+i \hat{m}_{a}\right)\right]} d u_{I}\right) \\
= & \frac{1}{k !}(2 i \sin (\pi z))^{-k} \oint \prod_{I=1}^{k} d u_{I} \prod_{I \neq J}^{k} \frac{\sin \left[-\pi\left(u_{I}-u_{J}\right)\right]}{\sin \left[\pi\left(u_{I}-u_{J}-z\right)\right]} \prod_{I, J}^{k} \frac{\sin \left[-\pi\left(u_{I}-u_{J}+\frac{R_{\mathrm{adj}}}{2} z-z\right)\right]}{\sin \left[\pi\left(u_{I}-u_{J}+\frac{R_{\mathrm{adj}}}{2} z\right)\right]} \\
& \times \prod_{I=1}^{k}\left(\prod_{j=1}^{N} \frac{\sin \left[-\pi\left(u_{I}-i \hat{\sigma}_{j}-z\right)\right]}{\sin \left[\pi\left(u_{I}-i \hat{\sigma}_{j}\right)\right]} \prod_{a=1}^{M} \frac{\sin \left[-\pi\left(-u_{I}+i \hat{m}_{a}-z\right)\right]}{\sin \left[\pi\left(-u_{I}+i \hat{m}_{a}\right)\right]}\right),
\end{aligned}
$$

where the integration contour is defined by picking the residues of the poles from the fundamental chiral mutliplets factors, namely the factors in the product $\prod_{j=1}^{N}$ and from "half" of the adjoint chiral multiplet factors $\prod_{I, J}^{k}$ according to the $\mathrm{JK}-\mathrm{Res}_{\zeta}$ prescription with $\zeta<0$. Concretely the integral is a sum over residues, each contribution corresponding to a pole $u^{*}=\left\{u_{I}^{*}\right\}_{1 \leq I \leq k}$ described by taking a decomposition of $k$ into $N$ non-negative integers, $k=\sum_{i=1}^{N} k_{i}, k_{i} \geq 0$, and picking $u_{I}^{*} \rightarrow u_{i, s_{i}}^{*}=i \hat{\sigma}_{i}-s_{i} \frac{R_{\text {adj }}}{2} z$ with $s_{i}=0, \cdots, k_{i}-1$. The arrow $\rightarrow$ indicates a mapping between the index $I$ into the index $\left(i, s_{i}\right)$. The residue contribution coming from a given pole $u^{*}=\left\{u_{i, s_{i}}^{*}\right\}$ arises with $k$ ! degeneracy, associated to permutations of the $u_{I}^{*}$. The partition function is then given by a sum over the residue contributions $\mathcal{I}_{\left(k_{i}\right)}$ associated to possible decompositions $k=\sum_{i=1}^{N} k_{i}$, counted with $k$ ! degeneracy:

$$
\mathcal{I}=\sum_{\substack{k_{1}, \cdots, k_{N} \geq 0 \\ \sum_{i=1}^{N} k_{i}=k}} \mathcal{I}_{\left(k_{i}\right)}
$$

The explicit evaluation of the residues leads to

$$
\begin{aligned}
& \mathcal{I}_{\left(k_{i}\right)} \\
& =(-1)^{(N+M) k} \prod_{i \neq j}^{N} \prod_{s=0}^{k_{j}-1} \frac{\operatorname{sh}\left[\hat{\sigma}_{i}-\hat{\sigma}_{j}+i \frac{R_{\mathrm{adj}}}{2}\left(k_{i}-s-1\right) z\right]}{\operatorname{sh}\left[\hat{\sigma}_{i}-\hat{\sigma}_{j}+i \frac{R_{\mathrm{adj}}}{2}\left(k_{i}-s-1\right) z+i z\right]} \prod_{i=1}^{N} \prod_{s=0}^{k_{i}-1} \prod_{a=1}^{M} \frac{\operatorname{sh}\left[\hat{\sigma}_{i}-\hat{m}_{a}+i \frac{R_{\mathrm{adj}}}{2} s z-i z\right]}{\operatorname{sh}\left[\hat{\sigma}_{i}-\hat{m}_{a}+i \frac{R_{\mathrm{adj}}}{2} s z\right]} \\
& \prod_{i \neq j}^{N} \prod_{s=0}^{k_{i}-1} \frac{\operatorname{sh}\left[\hat{\sigma}_{i}-\hat{\sigma}_{j}+i \frac{R_{\mathrm{adj}}}{2} s z+i z\right]}{\operatorname{sh}\left[\hat{\sigma}_{i}-\hat{\sigma}_{j}+i \frac{R_{\mathrm{adj}}}{2} s z\right]} \prod_{i \neq j}^{N} \prod_{s=0}^{k_{i}-1} \frac{\operatorname{sh}\left[\hat{\sigma}_{i}-\hat{\sigma}_{j}+i \frac{R_{\mathrm{adj}}}{2}(s+1) z-i z\right]}{\operatorname{sh}\left[\hat{\sigma}_{i}-\hat{\sigma}_{j}+i \frac{R_{\mathrm{adj}}}{2}(s+1) z\right]} \prod_{i=1}^{N} \prod_{s=0}^{k_{i}-1} \frac{\operatorname{sh}\left[i \frac{R_{\mathrm{adj}}}{2}(s+1) z-i z\right]}{\operatorname{sh}\left[i \frac{R_{\mathrm{adj}}}{2}(s+1) z\right]} .
\end{aligned}
$$

If we plug the adjoint R-charge $R_{\text {adj }}=2$ then the result simplifies to

$$
\mathcal{I}_{\left(k_{i}\right)}=(-1)^{(N+M) k} \prod_{i \neq j}^{N} \frac{\operatorname{sh}\left[\hat{\sigma}_{i}-\hat{\sigma}_{j}+i\left(k_{i}-k_{j}\right) z\right]}{\operatorname{sh}\left[\hat{\sigma}_{i}-\hat{\sigma}_{j}+i k_{i} z\right]} \prod_{i=1}^{N} \prod_{a=1}^{M} \frac{\operatorname{sh}\left[\hat{\sigma}_{i}-\hat{m}_{a}-i z\right]}{\operatorname{sh}\left[\hat{\sigma}_{i}-\hat{m}_{a}+i\left(k_{i}-1\right) z\right]} \prod_{i=1}^{N} \frac{\operatorname{sh}[\epsilon]}{\operatorname{sh}\left[i k_{i} z+\epsilon\right]}
$$



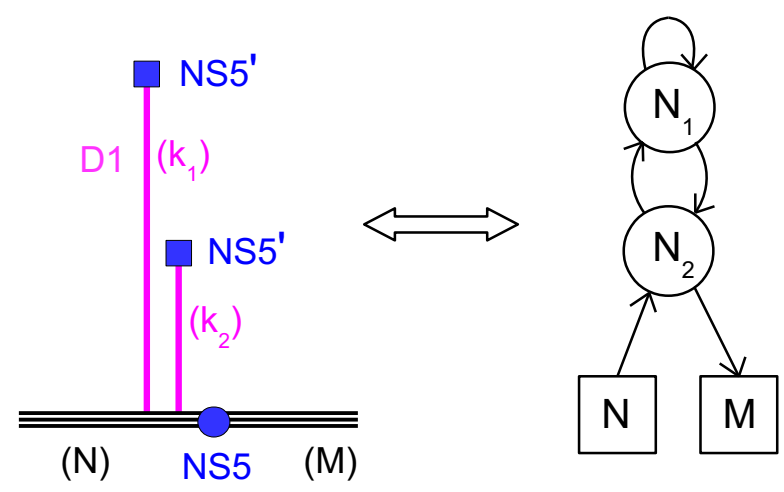

Figure 40. Brane realization of a Vortex loop associated with the representation $\mathcal{A}_{k_{1}} \otimes \mathcal{A}_{k_{2}}$ and SQM two-node quiver read by moving the D1-branes to the NS5-brane on the right. Here $N_{1}=k_{1}$ and $N_{2}=k_{1}+k_{2}$.

where we have introduced a regulating mass parameter $\epsilon$ for vanishing factors. ${ }^{75}$ Let us write $\mathcal{I}_{\epsilon}(z)=\prod_{i=1}^{N} \frac{\mathrm{sh}[\epsilon]}{\operatorname{sh}\left[i k_{i} z+\epsilon\right]}$. In the limit $z \rightarrow 1$ (at finite $\epsilon$ ) this factor become trivial.

Using the contraints $r_{+}+r_{-}=2$ and $q_{+}+q_{-}=1 / 2$, we obtain

$$
\mathcal{I}_{\left(k_{i}\right)}=(-1)^{(N+M) k} \prod_{i \neq j}^{N} \frac{\operatorname{sh}\left[\sigma_{i}-\sigma_{j}+i\left(k_{i}-k_{j}\right) z\right]}{\operatorname{sh}\left[\sigma_{i}-\sigma_{j}+i k_{i} z\right]} \prod_{i=1}^{N} \prod_{a=1}^{M} \frac{\operatorname{ch}\left[\sigma_{i}-m_{a}\right]}{\operatorname{ch}\left[\sigma_{i}-m_{a}+i k_{i} z\right]} \mathcal{I}_{\epsilon}(z)
$$

Here again the result matches the formula (5.45) giving the index as a sum over the weights of the representation $\mathcal{S}_{k}$ associated to the $1 \mathrm{~d} \mathcal{N}=4$ theory.

\section{B.3 Two-node quiver}

We consider a $\mathrm{U}\left(N_{1}\right) \times \mathrm{U}\left(N_{2}\right)$ quiver gauge theory with bifundamental chiral multiplets of R-charges $\widehat{r}, \widetilde{r}$ and $G_{F}$-charges $\widehat{q}, \widetilde{q}$. In addition we have $N \mathrm{U}\left(N_{2}\right)$-fundamental chiral multiplets with real masses $-\sigma_{j}$, R-charges $r_{+}$and $G_{F}$-charge $q_{+}, M \mathrm{U}\left(N_{2}\right)$-anti-fundamental chiral multiplets with masses $m_{a}, \mathrm{R}$-charges $r_{-}$and $G_{F}$-charge $q_{-}$and an adjoint chiral multiplet with R-charge $R_{\text {adj }}=0$ and $G_{F}$-charge $q_{\text {adj }}=-1$. The charges obey the superpotential constraints $r_{-}+r_{+}=2, q_{-}+q_{+}=1 / 2, \widehat{r}+\widetilde{r}+R_{\text {adj }}=2$ and $\widehat{q}+\widetilde{q}=1$. The FI parameters are both taken negative $\zeta_{1}, \zeta_{2}<0$. We also assume $N_{2}>N_{1}$. This setup corresponds to the $1 \mathrm{~d} \mathcal{N}=4$ theory obtained by moving the D1-branes to right NS5-brane, with $k_{1}=N_{1}$ D1-branes ending on an extra NS5'-brane and $k_{2}=N_{2}-N_{1}$ D1-branes ending on a second extra NS5'-brane (see figure 40). The representation associated to this quiver is $\mathcal{R}=\mathcal{A}_{k_{1}} \otimes \mathcal{A}_{k_{2}}$.

We introduce the complex parameters $\hat{\sigma}_{j}=\sigma_{j}+i q_{+}+i \frac{r_{+}}{2} z$ and $\hat{m}_{a}=m_{a}-i q_{-}-i \frac{r_{-}}{2} z$. in order to avoid higher order poles in the computation we keep $R_{\text {adj }}$ generic and only set

\footnotetext{
${ }^{75} \epsilon$ can be introduced as a very small real mass for the adjoint chiral multiplet. In this case $\epsilon$ would appear in the other factors as well. We do not introduce it in the other factors since it would complicate significantly the discussion, without changing the final result in the $3 \mathrm{~d} / 1 \mathrm{~d}$ matrix models.
} 
it to 0 at the end. The partition function or index is given by

$$
\begin{aligned}
\mathcal{I} & =\oint \prod_{I=1}^{N_{1}} d u_{I} \prod_{J=1}^{N_{2}} d v_{J} \frac{1}{N_{1} ! N_{2} !}\left(\frac{\pi}{\sin (\pi z)}\right)^{N_{1}+N_{2}} \prod_{I \neq J}^{N_{1}} \frac{\sin \left[-\pi\left(u_{I}-u_{J}\right)\right]}{\sin \left[\pi\left(u_{I}-u_{J}-z\right)\right]} \prod_{I \neq J}^{N_{2}} \frac{\sin \left[-\pi\left(v_{I}-v_{J}\right)\right]}{\sin \left[\pi\left(v_{I}-v_{J}-z\right)\right]} \\
& \prod_{I, J}^{N_{1}} \frac{\sin \left[-\pi\left(u_{I}-u_{J}+\frac{R_{\mathrm{adj}}}{2} z-z\right)\right]}{\sin \left[\pi\left(u_{I}-u_{J}+\frac{R_{\mathrm{adj}}}{2} z\right)\right]} \prod_{I=1}^{N_{1}}\left(\prod_{j=1}^{N} \frac{\sin \left[-\pi\left(v_{I}-i \hat{\sigma}_{j}-z\right)\right]}{\sin \left[\pi\left(v_{I}-i \hat{\sigma}_{j}\right)\right]} \prod_{a=1}^{M} \frac{\sin \left[-\pi\left(-v_{I}+i \hat{m}_{a}-z\right)\right]}{\sin \left[\pi\left(-v_{I}+i \hat{m}_{a}\right)\right]}\right) \\
& \prod_{I}^{N_{1}} \prod_{J}^{N_{2}} \frac{\sin \left[-\pi\left(u_{I}-v_{J}+\widehat{q}+\frac{\widehat{r}}{2} z-z\right)\right]}{\sin \left[\pi\left(u_{I}-v_{J}+\widehat{q}+\frac{\widehat{r}}{2} z\right)\right]} \frac{\sin \left[-\pi\left(v_{J}-u_{I}+\widetilde{q}+\frac{\widetilde{r}}{2} z-z\right)\right]}{\sin \left[\pi\left(v_{J}-u_{I}+\widetilde{q}+\frac{\widetilde{r}}{2} z\right)\right]} .
\end{aligned}
$$

The integration contour picks the residues at the poles selected by the JK-recipe as explained in the main text. The recipe allows to take poles from the the fundamental chiral mulitplet factors, as well as from "half" of the bifundamental and adjoint chiral multiplet factors. However one can realize, for instance, that picking a pole $u_{I}^{*}=u_{J}^{*}-\frac{R_{\text {adj }}}{2} z$ from the adjoint factor and a pole $u_{J}^{*}=v_{K}^{*}-\widehat{q}-\frac{\widehat{r}}{2} z$ from the bifundamental factor, leads to $u_{I}^{*}=v_{K}^{*}+\widetilde{q}+\frac{\widetilde{r}}{2} z-z-1$, where we have used the superpotential constraints, and in this case $u_{I}^{*}$ has an extra zero from the bifundamental factor, canceling the pole from the adjoint factor. This kind of reasoning leads to the conclusion that we cannot take a pole from the adjoint factor, as it yields a vanishing contribution.

A careful analysis along these lines leads to the following sets of poles $\left(u^{*}, v^{*}\right)$ contributing to $\mathcal{I}$ :

- With $k_{1}=N_{1}$ and $k_{2}=N_{2}-N_{1}$, a pole $\left\{u_{I}^{*}, v_{J}^{*}\right\}$ is characterized by a choice of decomposition

$$
k_{1}=\sum_{j=1}^{N} k_{j}^{(1)}, \quad k_{2}=\sum_{j=1}^{N} k_{j}^{(2)}, \quad \text { with } \quad k_{j}^{(\alpha)} \in\{0,1\} .
$$

- The explicit single-integral poles are given by

$$
\begin{aligned}
& u_{I}^{*} \rightarrow i \hat{\sigma}_{j}-s_{j}\left(\widehat{q}+\frac{\widehat{r}}{2}\right) \quad \text { for } \quad 0 \leq s_{j} \leq k_{j}^{(1)}, \\
& v_{J}^{*} \rightarrow i \hat{\sigma}_{j}-s_{j}\left(\widehat{q}+\widetilde{q}+\frac{\widehat{r}+\widetilde{r}}{2}\right) \text { for } \quad 0 \leq s_{j} \leq k_{j}^{(1)}+k_{j}^{(2)},
\end{aligned}
$$

with $\rightarrow$ denoting a mapping between the relevant indices. The range of the $s_{j}$ is such that it correctly gives the $N_{1} u_{I}^{*}$-poles and the $N_{2} v_{I}^{*}$-poles.

- Each residue contribution to the index appears with the degeneracy $k_{1} !\left(k_{1}+k_{2}\right)$ ! = $N_{1} ! N_{2} !$

The evaluation of the index gives

$$
\begin{aligned}
& \mathcal{I}=\sum_{k_{j}^{(1)} \in\{0,1\}} \sum_{k_{j}^{(2)} \in\{0,1\}} \mathcal{I}_{\left(k^{(1)}, k^{(2)}\right)}, \\
& \sum_{j}^{N} k_{j}^{(1)}=k_{1} \quad \sum_{j}^{N} k_{j}^{(2)}=k_{2}
\end{aligned}
$$


with

$$
\mathcal{I}_{\left(k^{(1)}, k^{(2)}\right)}=\mathcal{F}\left(\sigma_{i}, z\right) \prod_{a=1}^{M} \prod_{j=1}^{N} \prod_{s_{j}=0}^{k_{j}^{(1)}+k_{j}^{(2)}-1} \frac{\operatorname{ch}\left[\sigma_{j}-m_{a}+i\left(1-\frac{R_{\mathrm{adj}}}{2}\right) s_{j} z\right]}{\operatorname{ch}\left[\sigma_{j}-m_{a}+i\left(1-\frac{R_{\mathrm{adj}}}{2}\right) s_{j} z+i z\right]},
$$

where we have used the superpotential constraints. $\mathcal{F}\left(\sigma_{i}, z\right)$ is a complicated expression which will not be relevant for our analysis of mirror symmetry. It is expressed as a product of the form

$$
\mathcal{F}\left(\sigma_{i}, z\right)=\prod_{\alpha, \kappa} \prod_{i \neq j}^{N} \frac{\sin \left[-\pi\left(i \sigma_{i}-i \sigma_{j}+\alpha z \pm z+\kappa / 2\right)\right]}{\sin \left[\pi\left(i \sigma_{i}-i \sigma_{j}+\alpha z+\kappa / 2\right)\right]},
$$

where $\alpha, \kappa$ take real values. As in (B.10), there are terms which require a regularization by a small mass deformation $\epsilon$ and which evaluates to \pm 1 as $z \rightarrow 1$.

Plugging the adjoint $\mathrm{R}$-charge $R_{\mathrm{adj}}=0$, the result simplifies to

$$
\mathcal{I}_{\left(k^{(1)}, k^{(2)}\right)}=\mathcal{F}\left(\sigma_{i}, z\right) \prod_{a=1}^{M} \prod_{j=1}^{N} \frac{\operatorname{ch}\left[\sigma_{j}-m_{a}\right]}{\operatorname{ch}\left[\sigma_{j}-m_{a}+i\left(k_{j}^{(1)}+k_{j}^{(2)}\right) z\right]} .
$$

This result reproduces correctly (5.45) as a sum over the weights of the representation $\mathcal{A}_{k_{1}} \otimes \mathcal{A}_{k_{2}}$.

Open Access. This article is distributed under the terms of the Creative Commons Attribution License (CC-BY 4.0), which permits any use, distribution and reproduction in any medium, provided the original author(s) and source are credited.

\section{References}

[1] K.G. Wilson, Confinement of Quarks, Phys. Rev. D 10 (1974) 2445 [InSPIRE].

[2] K.A. Intriligator and N. Seiberg, Mirror symmetry in three-dimensional gauge theories, Phys. Lett. B 387 (1996) 513 [hep-th/9607207] [INSPIRE].

[3] A. Kapustin, B. Willett and I. Yaakov, Exact results for supersymmetric abelian vortex loops in 2+1 dimensions, JHEP 06 (2013) 099 [arXiv: 1211.2861] [INSPIRE].

[4] N. Drukker, T. Okuda and F. Passerini, Exact results for vortex loop operators in $3 d$ supersymmetric theories, JHEP 07 (2014) 137 [arXiv:1211.3409] [INSPIRE].

[5] B. Assel, J. Gomis, and R.C. Santamaria, unpublished.

[6] N. Drukker, J. Gomis and D. Young, Vortex Loop Operators, M2-branes and Holography, JHEP 03 (2009) 004 [arXiv: 0810.4344] [InSPIRE].

[7] G.W. Moore and N. Seiberg, Taming the Conformal Zoo, Phys. Lett. B 220 (1989) 422 [INSPIRE].

[8] J. Gomis and B. Le Floch, M2-brane surface operators and gauge theory dualities in Toda, arXiv: 1407.1852 [INSPIRE].

[9] A. Hanany and E. Witten, Type IIB superstrings, BPS monopoles and three-dimensional gauge dynamics, Nucl. Phys. B 492 (1997) 152 [hep-th/9611230] [InSPIRE].

[10] J. Gomis and F. Passerini, Holographic Wilson Loops, JHEP 08 (2006) 074 [hep-th/0604007] [INSPIRE]. 
[11] J. Gomis and F. Passerini, Wilson Loops as D3-branes, JHEP 01 (2007) 097 [hep-th/0612022] [INSPIRE].

[12] J.M. Maldacena, Wilson loops in large-N field theories, Phys. Rev. Lett. 80 (1998) 4859 [hep-th/9803002] [INSPIRE].

[13] S.-J. Rey and J.-T. Yee, Macroscopic strings as heavy quarks in large- $N$ gauge theory and anti-de Sitter supergravity, Eur. Phys. J. C 22 (2001) 379 [hep-th/9803001] [INSPIRE].

[14] A. Kapustin, B. Willett and I. Yaakov, Nonperturbative Tests of Three-Dimensional Dualities, JHEP 10 (2010) 013 [arXiv: 1003.5694] [INSPIRE].

[15] S. Benvenuti and S. Pasquetti, 3D-partition functions on the sphere: exact evaluation and mirror symmetry, JHEP 05 (2012) 099 [arXiv:1105.2551] [INSPIRE].

[16] B. Assel, Hanany-Witten effect and $\mathrm{SL}(2, \mathbb{Z})$ dualities in matrix models, JHEP 10 (2014) 117 [arXiv: 1406.5194] [INSPIRE].

[17] A. Kapustin, B. Willett and I. Yaakov, Exact Results for Wilson Loops in Superconformal Chern-Simons Theories with Matter, JHEP 03 (2010) 089 [arXiv:0909.4559] [INSPIRE].

[18] K. Hori, H. Kim and P. Yi, Witten Index and Wall Crossing, JHEP 01 (2015) 124 [arXiv: 1407.2567] [INSPIRE].

[19] C. Cordova and S.-H. Shao, An Index Formula for Supersymmetric Quantum Mechanics, arXiv: 1406.7853 [INSPIRE].

[20] N. Seiberg, Electric-magnetic duality in supersymmetric nonAbelian gauge theories, Nucl. Phys. B 435 (1995) 129 [hep-th/9411149] [INSPIRE].

[21] A. Gadde and S. Gukov, 2d Index and Surface operators, JHEP 03 (2014) 080 [arXiv: 1305.0266] [INSPIRE].

[22] E.I. Buchbinder, J. Gomis and F. Passerini, Holographic gauge theories in background fields and surface operators, JHEP 12 (2007) 101 [arXiv:0710.5170] [INSPIRE].

[23] K. Zarembo, Supersymmetric Wilson loops, Nucl. Phys. B 643 (2002) 157 [hep-th/0205160] [INSPIRE].

[24] L. Rozansky and E. Witten, HyperKähler geometry and invariants of three manifolds, Selecta Math. 3 (1997) 401 [hep-th/9612216] [INSPIRE].

[25] A. Kapustin and E. Witten, Electric-Magnetic Duality And The Geometric Langlands Program, Commun. Num. Theor. Phys. 1 (2007) 1 [hep-th/0604151] [InSPIRE].

[26] S. Gukov and E. Witten, Gauge Theory, Ramification, And The Geometric Langlands Program, hep-th/0612073 [INSPIRE].

[27] N.R. Constable, J. Erdmenger, Z. Guralnik and I. Kirsch, Intersecting D-3 branes and holography, Phys. Rev. D 68 (2003) 106007 [hep-th/0211222] [INSPIRE].

[28] D. Gaiotto, Surface Operators in $N=24$ d Gauge Theories, JHEP 11 (2012) 090 [arXiv:0911.1316] [INSPIRE].

[29] D. Gaiotto and E. Witten, S-duality of Boundary Conditions In $N=4$ Super Yang-Mills Theory, Adv. Theor. Math. Phys. 13 (2009) 721 [arXiv:0807.3720] [INSPIRE].

[30] B. Assel, C. Bachas, J. Estes and J. Gomis, Holographic Duals of $D=3 N=4$ Superconformal Field Theories, JHEP 08 (2011) 087 [arXiv:1106.4253] [INSPIRE]. 
[31] B. Assel, C. Bachas, J. Estes and J. Gomis, IIB Duals of D $=3 N=4$ Circular Quivers, JHEP 12 (2012) 044 [arXiv: 1210.2590] [INSPIRE].

[32] J. de Boer, K. Hori, H. Ooguri and Y. Oz, Mirror symmetry in three-dimensional gauge theories, quivers and D-branes, Nucl. Phys. B 493 (1997) 101 [hep-th/9611063] [InSPIRE].

[33] N. Drukker and B. Fiol, All-genus calculation of Wilson loops using D-branes, JHEP 02 (2005) 010 [hep-th/0501109] [INSPIRE].

[34] S. Yamaguchi, Wilson loops of anti-symmetric representation and D5-branes, JHEP 05 (2006) 037 [hep-th/0603208] [INSPIRE].

[35] U. Danielsson, G. Ferretti and I.R. Klebanov, Creation of fundamental strings by crossing D-branes, Phys. Rev. Lett. 79 (1997) 1984 [hep-th/9705084] [INSPIRE].

[36] C.P. Bachas, M.B. Green and A. Schwimmer, $(8,0)$ quantum mechanics and symmetry enhancement in type-I' superstrings, JHEP 01 (1998) 006 [hep-th/9712086] [INSPIRE].

[37] C.G. Callan and J.M. Maldacena, Brane death and dynamics from the Born-Infeld action, Nucl. Phys. B 513 (1998) 198 [hep-th/9708147] [INSPIRE].

[38] H. Kim, S.-J. Lee and P. Yi, Mutation, Witten Index and Quiver Invariant, JHEP 07 (2015) 093 [arXiv: 1504.00068] [INSPIRE].

[39] I.B. Samsonov and D. Sorokin, Superfield theories on $S^{3}$ and their localization, JHEP 04 (2014) 102 [arXiv: 1401.7952] [INSPIRE].

[40] G. Festuccia and N. Seiberg, Rigid Supersymmetric Theories in Curved Superspace, JHEP 06 (2011) 114 [arXiv: 1105.0689] [INSPIRE].

[41] J. Gomis and N. Ishtiaque, Kähler potential and ambiguities in $4 d \mathcal{N}=2$ SCFTs, JHEP 04 (2015) 169 [arXiv:1409.5325] [INSPIRE].

[42] N. Hama, K. Hosomichi and S. Lee, Notes on SUSY Gauge Theories on Three-Sphere, JHEP 03 (2011) 127 [arXiv: 1012.3512] [INSPIRE].

[43] D.L. Jafferis, The Exact Superconformal R-Symmetry Extremizes Z, JHEP 05 (2012) 159 [arXiv: 1012.3210] [INSPIRE].

[44] N. Hama, K. Hosomichi and S. Lee, SUSY Gauge Theories on Squashed Three-Spheres, JHEP 05 (2011) 014 [arXiv:1102.4716] [INSPIRE].

[45] E. Witten, Constraints on Supersymmetry Breaking, Nucl. Phys. B 202 (1982) 253 [INSPIRE].

[46] L.C. Jeffrey and F.C. Kirwan, Localization for nonabelian group actions, Topology 34 (1995) 291327 [alg-geom/9307001].

[47] T. Nishioka, Y. Tachikawa and M. Yamazaki, $3 d$ Partition Function as Overlap of Wavefunctions, JHEP 08 (2011) 003 [arXiv: 1105.4390] [INSPIRE].

[48] C. Closset, T.T. Dumitrescu, G. Festuccia, Z. Komargodski and N. Seiberg, Contact Terms, Unitarity and F-Maximization in Three-Dimensional Superconformal Theories, JHEP 10 (2012) 053 [arXiv:1205.4142] [INSPIRE].

[49] C. Closset, T.T. Dumitrescu, G. Festuccia, Z. Komargodski and N. Seiberg, Comments on Chern-Simons Contact Terms in Three Dimensions, JHEP 09 (2012) 091 [arXiv: 1206.5218] [INSPIRE].

[50] M. Bullimore, M. Fluder, L. Hollands and P. Richmond, The superconformal index and an elliptic algebra of surface defects, JHEP 10 (2014) 62 [arXiv:1401.3379] [INSPIRE]. 